\title{
Geometrically Nonlinear Stress Recovery in Composite Laminates
}

\author{
Timothy Benjamin Hartman
}

\begin{abstract}
Dissertation submitted to the Faculty of the
Virginia Polytechnic Institute and State University

in partial fulfillment of the requirements for the degree of
\end{abstract}

Doctor of Philosophy

in

Engineering Mechanics

Scott W. Case, Co-Chair

Michael W. Hyer, Co-Chair

Romesh C. Batra

Shane D. Ross

Robert L. West

16 April 2013

Blacksburg, Virginia

Keywords: composite laminate, stress recovery, geometrically nonlinear, transverse stress, interlaminar stress

Copyright (c) 2013 T.B. Hartman 


\title{
Geometrically Nonlinear Stress Recovery in Composite Laminates
}

\author{
Timothy B. Hartman
}

\section{(ABSTRACT)}

Composite laminates are increasingly being used as primary load bearing members in structures. However, because of the directional dependence of the properties of composite materials, additional failure modes appear that are absent in homogeneous, isotropic materials. Therefore, a stress analysis of a composite laminate is not complete without an accurate representation of the transverse (out-of-plane) stresses.

Stress recovery is a common method to estimate the transverse stresses from a plate or shell analysis. This dissertation extends stress recovery to problems in which geometric nonlinearities, in the sense of von Kármán, are important. The current work presents a less complex formulation for the stress recovery procedure for plate geometries, compared with other implementations, and results in a post-processing procedure which can be applied to data from any plate analyses; analytical or numerical methods, resulting in continuous or discretized data.

Recovered transverse stress results are presented for a variety of geometrically nonlinear example problems: a semi-infinite plate subjected to quasi-static transverse and shear loading, and a finite plate subjected to both quasi-static and dynamic transverse loading. For all cases, the corresponding results from a fully three-dimensional stress analysis are shown alongside the distributions from the stress recovery procedure. Good agreement is observed between the stresses obtained from each method for the cases considered. Discussion is included regarding the applicability and accuracy of the technique to varying plate geometries and varying degrees of nonlinearity, as well as the viability of the procedure in replacing a three-dimensional analysis in regard to the time required to obtain a solution.

The proposed geometrically nonlinear stress recovery procedure results in estimations for transverse stresses which show good correlation to the three-dimensional finite element solutions. The procedure is accurate for quasi-static and dynamic loading cases and proves to be a viable replacement for more computationally expensive analyses. 
To the girl who joined me on this journey

who will always love me

never leave me

who encourages me

challenges me

grows me

follows me

我爱你。 


\section{Acknowledgements}

In the course of my time in Blacksburg, I have indebted myself to many mentors, colleagues, peers, and friends. Special thanks to:

Prof. Scott Case, for five years of guiding, encouraging, questioning, and correcting. You gave me room to fail and room to investigate areas you knew would not work. And you pushed me out of this nest when you knew it was time. Thanks for providing an opportunity for me to lead a course, and giving a reading eye and listening ear so close to deadlines.

Prof. Mike Hyer, for giving endless hours of help, in your retirement nonetheless. For introducing me to people I need to know. For always having my back. For reading through papers ten times and listening to presentations more than that. For telling me flat out when I went astray. For instructing Plates \& Shells in an atmosphere that was unintimidating socially and challenging academically. For demonstrating a care for undergraduate and graduate students and a brilliance in research at the same time. For overall being an awesome mentor.

Profs. Romesh Batra, Shane Ross, and Bob West, for serving on my advisory committee, and not taking it easy on me.

Sponsors and colleagues at Pratt \& Whitney, for providing finincial support during the course of this investigation. Special thanks to Sreenivasa Voleti and Rajiv Naik for the encouragement and lively discussion during these years.

The Case group, an ecclectic mix of interests under the purview of composite materials that never leaves me amazed at how much knowledge is yet to be had.

Jess Dibelka and Frances Davis, for making the lab a great place to be. Thanks for the encouragement and unending help, even after thousands of questions.

Pierce Umberger, for your friendship, conversation, and endless supply of manufacturing and computing knowledge. And for introducing me to XKCD.

David Allen and Matt Yaple, for working with me and allowing me practice in guiding you.

Bev Williams, for always keeping track of Jess and me and for the many insights.

Dave Simmons and Darrell Link, for managing the best shop I've seen. Your constant patience, superior knowledge, selfless help, and care for precision was not lost on me. 
Tim Tomlin, for managing the ESM compute cluster. One unscheduled downtime in three years with no data loss certainly made my studies easier.

The ESM staff. I've not met a more helpful and supportive group at any other school. You make Virginia Tech stand out.

ESM 2204 13165, for being my first class and helping me confirm that I want to teach.

Blacksburg Christian Fellowship, for being a constant source of friendship, encouragement, and Truth.

Many Blacksburg friends, including the Liles, Whites, Paulettes, Jaasmas, Robinsons, Schuberts, and McCarthys. You all are our best friends. 


\section{Contents}

Front Matter

Title Page . . . . . . . . . . . . . . . . . . . . . . i

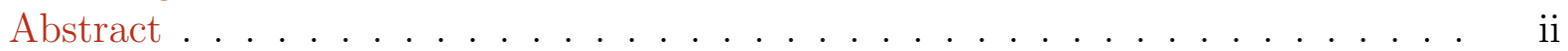

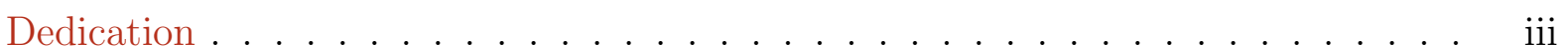

Acknowledgements . . . . . . . . . . . . . . . . . iv

Table of Contents . . . . . . . . . . . . . . . . . . vi

List of Figures . . . . . . . . . . . . . . . . . . . . . ix

List of Tables . . . . . . . . . . . . . . . . . . . . xi

1 Introduction $\quad 1$

2 Background $\quad 4$

2.1 The History of Plate Theory of Composites . . . . . . . . . . . . . . 4

2.1.1 Anisotropic Plate Theory . . . . . . . . . . . . . . . . 4

2.1.2 Classical Laminated Plate Theory . . . . . . . . . . . . . . . . . 5

2.1.3 Shear-Deformable Plate Theory . . . . . . . . . . . . . 7

2.1.4 Higher-Order Theories . . . . . . . . . . . . . . . . 8

2.1.5 Layerwise Theories . . . . . . . . . . . . . . . . . . . . 9

2.2 Determination of Interlaminar Stresses . . . . . . . . . . . . . . . . 10

2.2.1 Constitutive Equations . . . . . . . . . . . . . . . . . . . 11

2.2.2 Equilibrium Considerations-Stress Recovery . . . . . . . . . . . . . 12

2.2.2.1 Stress Recovery for Geometrically Linear Problems . . . . . 12

2.2.2.2 Stress Recovery for Geometrically Nonlinear Problems . . . 14

2.2.3 Numerical Methods . . . . . . . . . . . . . . . . . . . 17

2.3 Summary of Considerations for Geometric Nonlinearities . . . . . . . . . . . 19

3 Geometrically Nonlinear Stress Recovery 21

3.1 Geometrically Nonlinear Equilibrium Equations . . . . . . . . . . . . . 21

3.2 The Stress Recovery Procedure . . . . . . . . . . . . . . . . . . . 23

3.3 Application to Laminated Plates . . . . . . . . . . . . . . . . . . . 26

3.4 Application to Discretized Solutions . . . . . . . . . . . . . 28

3.4.1 Calculation of Terms of the Integrands . . . . . . . . . . . . . . . 30

3.4.2 Calculation of Rotations . . . . . . . . . . . . . . . . . . . 31

3.4.3 Calculations Using Piecewise Constant Stresses . . . . . . . . . . . . 31

3.4.4 Calculations Using Piecewise Linear Stresses . . . . . . . . . . . . . . 33

3.5 Contribution of Individual Terms . . . . . . . . . . . . . . . . . . . 36 
3.6 Measurement of Procedure Accuracy . . . . . . . . . . . . . . . . . . 36

4 Stress Recovery in a Semi-Infinite Domain 37

4.1 Description of Example Problems . . . . . . . . . . . . . . . . . . . 38

4.2 Simplification of Stress Recovery Procedure . . . . . . . . . . . . . . . . . 40

4.3 Results . . . . . . . . . . . . . . . . . . . . . 41

4.3.1 Problem Parameters . . . . . . . . . . . . . . . . . 41

4.3.1.1 Details of Plate Finite Element Model . . . . . . . . . . . . 42

4.3.1.2 Details of Benchmark 3D Finite Element Model . . . . . . . 43

4.3.1.3 Details of Closed-Form Plate Solutions . . . . . . . . . . . . 44

4.3.2 Stress Recovery Results . . . . . . . . . . . . . . . 45

4.3.2.1 Transverse Loading Case $\left(q_{0} \neq 0, t_{0}=0\right)$. . . . . . . . 46

4.3.2.2 Shear Loading Case $\left(q_{0}=0, t_{0} \neq 0\right) \ldots . . . . . . .51$

4.4 Discussion and Concluding Remarks . . . . . . . . . . . . . . . . 56

$5 \quad$ Stress Recovery in a Finite Domain $\quad 59$

5.1 Description of Solution Methods . . . . . . . . . . . . . . . 61

5.1.1 Finite Element Plate Solution . . . . . . . . . . . . . . . 61

5.1.2 Finite Element Benchmark 3D Solution . . . . . . . . . . . . . . 62

5.2 Results ............................. . . . . . . . . . . . . .

5.2.1 Summary of Plate Response . . . . . . . . . . . . . . . . 64

5.2.2 Recovery of Transverse Stresses . . . . . . . . . . . . . . . . 70

5.2.2.1 Recovered Stresses at Quarter-Point, Point A . . . . . . 73

5.2.2.2 Recovered Stresses near Plate Center, Point B . . . . . . . . 77

5.2.2.3 Recovered Stresses near $x^{+}$-Edge, Point C . . . . . . . . . . 79

5.2.2.4 Recovered Stresses near $y^{+}$-Edge, Point D . . . . . . . . . 79

5.2.3 Analysis of Convergence of Recovery Procedure . . . . . . . . . . . . 82

5.2.3.1 Convergence of Finite Element Results . . . . . . . . . . . . 82

5.2.3.2 Convergence of the Iteration Procedure . . . . . . . . . . . . 84

5.2.4 Analysis of Accuracy of Recovery Procedure . . . . . . . . . . . . . . 86

5.2.4.1 Accuracy with Varying Plate Span-Ratio . . . . . . . . . . . 86

5.2.4.2 Accuracy with Varying Plate Rotations . . . . . . . . . . . . 88

5.2.4.3 Accuracy with Varying Locations within Plate . . . . . . . . 90

5.2.4.4 Accuracy with Varying Loading Distributions . . . . . . . . 92

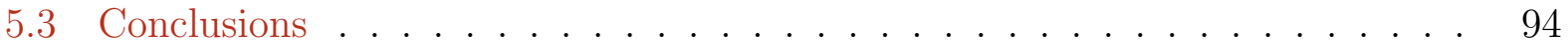

6 Stress Recovery Considering Inertial Effects $\quad 95$

6.1 Effect of Inertial Terms on Stress Recovery . . . . . . . . . . . . . . . . . 96

6.2 Plate Dynamic Response . . . . . . . . . . . . . . . . . . . . . . 98

6.3 Details of Finite Element Models . . . . . . . . . . . . . . . . . . . . . 99

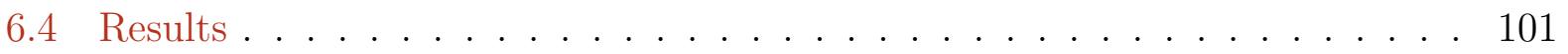

6.4.1 Ramp Loading . . . . . . . . . . . . . . . . . 101

6.4.2 Sinusoidal Loading . . . . . . . . . . . . . . . . . . 105

6.5 Computation Time of Stress Recovery . . . . . . . . . . . . . . 108

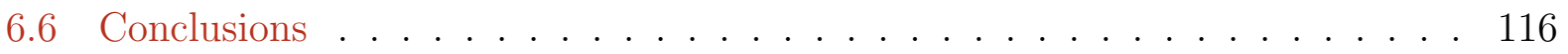


$\begin{array}{llr}7 \text { Conclusions } & 117\end{array}$

$\begin{array}{lr}\text { References } & 120\end{array}$

A Development of Geometrically Nonlinear Equilibrium Equations $\quad 126$

A.1 Notation of Coordinate Systems . . . . . . . . . . . . . . 126

A.2 Balance of Linear Momentum . . . . . . . . . . . . . . . . . . 128

A.3 Balance of Linear Momentum in Global Coordinates . . . . . . . . . . . . . 129

A.4 Motivation for Coordinate Transformation . . . . . . . . . . . . . . . 130

A.5 Transformation to Body-Oriented Coordinate System . . . . . . . . . . . . 131

A.5.1 Summary of Simplifications of Equilibrium Equations . . . . . . . . . 134

A.5.2 Simplification for Small Strains . . . . . . . . . . . . . . 135

A.5.3 Simplification for Moderate Rotations . . . . . . . . . . . . . . 136

A.5.4 Simplification to Classical Equilibrium Equations . . . . . . . . . . 136

B Semi-Infinite Closed-Form Plate Solutions $\quad 138$

B.1 Solutions for Loading Cases . . . . . . . . . . . . . . . . . . . . . . . . 140

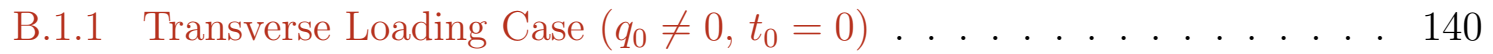

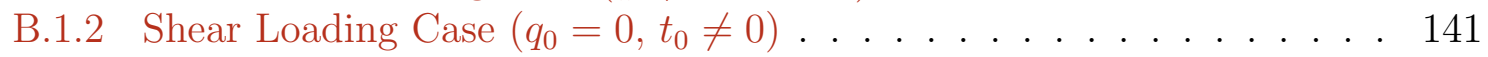

B.2 Details of Semi-Infinite Rayleigh-Ritz Solutions . . . . . . . . . . . . . . . 142

B.3 Application of the Stress Recovery Procedure . . . . . . . . . . . . . . . . 143

C Justification for Switching to Abaqus for Dynamic Plate Solutions $\quad 144$

D Permissions for Copyrighted Content $\quad 149$

D.1 Reprint of Pagano's Figure. . . . . . . . . . . . . . . . . . . . . . 150

D.2 Reprint of Jones' Figure. . . . . . . . . . . . . . . . . . . . . . . . 151 


\section{List of Figures}

2.1 Central plate deflection with varying span-ratios. . . . . . . . . . 6

2.2 Transverse shear stress distribution along edge of semi-infinite strip. . . . . . 8

3.1 Coordinate system definitions. . . . . . . . . . . . . . . . . . . 22

3.2 Notation of layer numbering and locations through the thickness of a laminated plate. . . . . . . . . . . . . . . . . 27

3.3 Example locations of known values from finite element solution. . . . . . . . 29

3.4 Example of Constant Data Distribution through Layers . . . . . . . . . . . 32

3.5 Example of Linear Data Distribution through Layers . . . . . . . . . . . . 34

4.1 View of semi-infinite plate. . . . . . . . . . . . . . . . . . . . . . 38

4.2 Cross section of semi-infinite plate. . . . . . . . . . . . . . . . 39

4.3 Deflection of isotropic plate. . . . . . . . . . . . . . . . 46

4.4 Interlaminar stresses at $x=0.05 \cdot a$ due to transverse loading. . . . . . . . 48

4.5 Interlaminar stresses at $x=0.45 \cdot a$ due to transverse loading. . . . . . . . . 52

4.6 Interlaminar stresses at $x=0.05 \cdot a$ due to shear loading. . . . . . . . . . . 53

4.7 Interlaminar stresses at $x=0.45 \cdot a$ due to shear loading. . . . . . . . . . 55

5.1 Simply supported plate. . . . . . . . . . . . . . . . . . . . 60

5.2 Deformation of nodes through the thickness at $x=a / 2, y=0$ due to enforced boundary condition. . . . . . . . . . . . . . . . 64

5.3 Force-displacement relationship for $a / H=60$ plate with varying mesh densities. 65

5.4 Plate displacement, to scale. . . . . . . . . . . . . . . . . . 65

5.5 Intralaminar stress distribution at center of quarter model. . . . . . . . . . 67

5.6 Convergence of intralaminar stresses for benchmark solution. . . . . . . . . . 68

5.7 Convergence of transverse stresses for benchmark solution. . . . . . . . . . 69

5.8 Locations of stress recovery results. . . . . . . . . . . . . . . . 70

5.9 Graphical representation of elements involved in iteration procedure. . . . . . 72

5.10 Results of stress recovery at Point A, the quarter-point. . . . . . . . . . . 74

5.11 Contribution of individual terms from Table 3.1 to $\tau_{x z}$ at quarter-point. . . . 76

5.12 Results of stress recovery at Point B, near plate center. . . . . . . . . . . 78

5.13 Results of stress recovery at Point C, near $x^{+}$-edge. . . . . . . . . . . . 80

5.14 Results of stress recovery at Point D, near ${ }^{+} y$-edge . . . . . . . . . . . . 81

5.15 Convergence of finite element results at Point A, the quarter-point. . . . . . 83

5.16 Convergence of iteration procedure at Point A, the quarter-point. . . . . . . 85

5.17 Error in recovered $\sigma_{z z}$ at top surface and plate rotations as a function of plate span-ratio. . . . . . . . . . . . . . . . . . 87 
5.18 Results of stress recovery at quarter-point of thick $(a / H=15)$ plate. . . . .

5.19 Error in recovered $\sigma_{z z}$ at top surface and rotations as a function of plate deflection. . . . . . . . . . . . . . . . . 90 90

5.20 Error in recovered $\sigma_{z z}$ at top surface for all elements, varying mesh density. .

5.21 Value and error of recovered $\sigma_{z z}$ for all elements under biharmonic transverse loading. . . . . . . . . . . . . . . . . . . . 93

6.1 Stress recovery of linear dynamic problem including inertial terms. . . . . . . 97

6.2 Linear natural frequency and period dependence on plate deflection. . . . . . 98

6.3 First four mode shapes of dynamic plate response. . . . . . . . . . . . . 100

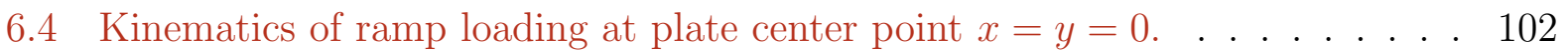

6.5 Results of stress recovery at Point A. . . . . . . . . . . . . . . 103

6.6 Results of stress recovery at Point A, the quarter-point. . . . . . . . . . . . 104

6.7 Three cycles of harmonic loading response. . . . . . . . . . . . . . . . . . 106

6.8 First period of harmonic loading response. . . . . . . . . . . . . . . 106

6.9 Plate deflection time-history. . . . . . . . . . . . . . . . . 107

6.10 Detail of plate center deflection time-history. . . . . . . . . . . . . . 107

6.11 Time sequence of transverse stresses at $f=943.7 \mathrm{~Hz} . \quad$. . . . . . . . . . . 109

A.1 Coordinate system definitions. . . . . . . . . . . . . . . . 127

A.2 Notation for stress. . . . . . . . . . . . . . . . . . . . 128

A.3 Notation for coordinate systems. . . . . . . . . . . . . . . . . . 129

C.1 Kinematics of ramp loading using various finite element analysis software. . . 145

C.2 Difference between solid and plate. . . . . . . . . . . . . . . 146

C.3 Difference between solid and plate at $y / b=0.25 \ldots \ldots \ldots$. . . . . . . . 147

C.4 Stress history at quarter-point. . . . . . . . . . . . . . . . . . 147

C.5 Stress distribution at quarter-point. . . . . . . . . . . . . . . . . 148 


\section{List of Tables}

3.1 Listing of Terms in Integrands of Eq. (3.14) $\ldots \ldots \ldots \ldots$

4.1 Listing of Terms in Integrands of Eq. (4.4) . . . . . . . . . . . . . 41

4.2 Material properties. . . . . . . . . . . . . . . . . . . . . 42

4.3 Plate parameters. . . . . . . . . . . . . . . . . . . . . . . . . . . 42

4.4 Solution nomenclature. . . . . . . . . . . . . . . . . . . . . . 45

4.5 Example problems solution kinematics. . . . . . . . . . . . . . . . . . 47

5.1 Summary of the properties of examined plate locations. . . . . . . . . . . 71

5.2 Details of solutions with varying span-ratios. . . . . . . . . . 88

6.1 Run time of stress recovery procedure for entire plate domain recovery. . . . 114

6.2 Run time of stress recovery procedure for single element recovery. . . . . . . 115

6.3 Profile of stress recovery implementation. . . . . . . . . . . . . . 115

A.1 Simplifications applied to equations of equilibrium. . . . . . . . . . . 134

B.1 Number of coefficients chosen for polynomials in Rayleigh-Ritz technique. . . 142

B.2 Coefficients of Rayleigh-Ritz solutions. . . . . . . . . . . . . . . . . 143 


\section{Chapter 1}

\section{Introduction}

Composite materials, those materials composed of two or more constituents, are being used more frequently as primary structural members in products ranging from those in the aerospace and automotive industries to civil and recreational applications. As primary members, structures made of composite materials must undergo a thorough stress analysis as part of the design process to ensure their reliability in service.

High-strength and high-stiffness fibers embedded in a matrix form a common composite material system. If the fibers are oriented in a preferred plane or direction, the bulk material will tend to have some degree of anisotropy. For example, the mechanical strength in the preferred plane or direction will differ, perhaps substantially, from the strength on other planes or in other directions. Additionally, structures made from these fibrous composites are commonly manufactured by stacking layers to form a laminate. In such structures, the layer interfaces are a common failure location, as normal and shear strengths transverse to the interface are generally much lower than the in-plane strengths of the laminate. Thus, when performing a stress analysis on a laminated composite structure, determining the stresses transverse to the layers is necessary. However, laminated composites are often used in plateor shell-type structures, so analyses using plate or shell theories are often used. Classical plate or shell theories grossly miscalculate the transverse stresses. Even the commonly used 
shear-deformable theories, which represent an attempt to predict the transverse stresses, misrepresent the distribution and magnitude of these stresses. Fully three-dimensional analyses of these structures, while more accurate than a plate or shell analysis, may either be intractable (in the case of analytical solutions) or more expensive computationally (in the case of numerical solutions). Hence, while it is desirable to use the plate or shell analyses for simplicity, some method is needed to at least estimate the transverse stresses.

The work herein presents the development of a technique to provide such an estimate. The technique is based on integrating the equilibrium equations from the theory of elasticity through the thickness of the laminate, using the stresses as computed by a plate or shell analysis. The technique is known as stress recovery, and it is applied to several example problems wherein geometric nonlinearities, in the sense of von Kármán, and dynamic effects are important.

Several authors have previously considered the ability of the stress recovery technique to estimate the transverse stresses in both geometrically linear and geometrically nonlinear problems. Their contributions are described in Chapter 2. The work described herein extends what is found in the literature by way of the following objectives:

1. Developing a formulation for the geometrically nonlinear stress recovery procedure that is less complex than other implementations presented in the literature.

2. Evaluating which terms of the geometrically nonlinear equilibrium equations are necessary for accurate stress recovery, as previous works present various simplifications.

3. Comparing results from the developed stress recovery procedure, for both quasi-static and dynamic example problems, to results obtained by a benchmark three-dimensional finite element analysis.

Chapter 3 presents the current formulation of the geometrically nonlinear stress recovery procedure for application to flat plates. Its application to solutions, such as a finite element solution, in which discretized data are obtained is discussed in Section 3.4. 
In Chapter 4, the developed geometrically nonlinear stress recovery procedure is first applied to the case of a semi-infinite plate under quasi-static transverse and shear loading. The semi-infinite extent of the plate provides simplifications which reduce the complexity of the stress recovery procedure, thereby providing example problems containing the necessary components to test the ability of the geometrically nonlinear stress recovery procedure to estimate transverse stresses without the algebraic overhead of the full equilibrium equations.

With the knowledge gained from the semi-infinite example problems, plates of finite dimensions under the influence of a quasi-static distributed pressure load are considered in Chapter 5. In addition to presenting the results of the transverse stress distribution at various locations within the finite plate, Section 5.2.2 also contains a discussion of the contribution of various algebraic terms in the geometrically nonlinear equilibrium equations to the distribution of the recovered transverse stresses. Finally, the convergence and accuracy of the stress recovery procedure are examined in Sections 5.2.3 and 5.2.4, respectively.

The effect of inertia on the geometrically nonlinear stress recovery procedure is considered in Chapter 6. The quasi-static pressure load of Chapter 5 is replaced with a time-varying pressure load. The comparison of the recovered transverse stresses and those obtained by a benchmark three-dimensional finite element analysis is reported in Section 6.4. The chapter includes, in Section 6.5, a discussion of the computation time required to apply an implementation of the developed geometrically nonlinear stress recovery procedure compared to the time required to obtain results from the fully three-dimensional analysis.

The work concludes in Chapter 7 with a summary of the findings in Chapters 4 to 6 and an analysis of the fulfillment of the objectives listed earlier in this chapter. 


\section{Chapter 2}

\section{Background}

\subsection{The History of Plate Theory of Composites}

\subsubsection{Anisotropic Plate Theory}

To the Western world, Lekhnitskii is often considered the first author who systematically introduced anisotropy into the study of solid mechanics.

So begin Tsai and Cheron in the preface to their translation of S.G. Lekhnitskii's second edition of Anisotropic Plates [1]. It is in Lekhnitskii's presentation of the theory of bending ${ }^{1}$ of anisotropic plates where a first mention of determining transverse stresses in a plate by integrating the equilibrium equations is found [1]. Lekhnitskii omits the transverse normal stress in the case of small deflections ("in any event, the deflections do not exceed the thickness" of the plate [1]). In the consideration of plates undergoing large deflections, a discussion of transverse stresses is altogether absent.

Lekhnitskii credits von Kármán ${ }^{2}$ with introducing the governing equations of isotropic plates undergoing finite rotations. The difficulty in solving such problems is the interaction between the in-plane forces and bending moments. If either one or the other exists, the

\footnotetext{
${ }^{1}$ Lekhnitskii credits M.T. Huber [2] as the main developer of the approximate theory of bending of anisotropic plates.

${ }^{2}$ von Kármán, Th., Encyclopedia of Mathematical Sciences, V. IV, 1910, p. 349
} 
governing equations for small deflections still hold; if both exist, the problem "is considerably more complicated," as the integration of the governing equation involves nonlinear terms. Lekhnitskii notes that the exact solution for even a simple problem involving large deflections is yet unknown ${ }^{3}$.

\subsubsection{Classical Laminated Plate Theory}

The plates studied by Lekhnitskii, though anisotropic, were homogeneous. The development of the mechanics of inhomogeneous, anisotropic materials was introduced ${ }^{4}$ by Dong et al. [4] in 1962, although Reissner and Stavsky [5] and Stavsky [6] developed the theory for laminated orthotropic plates in 1961.

Pagano developed ${ }^{5}$ exact (in the context of linear elasticity) solutions for simply-supported semi-infinite [9] and finite [10] plates and compared these solutions to those given by the CLPT (classical laminated plate theory). The work concludes that CLPT "leads to a very poor description of laminate response" for small span-ratios, but converges to the exact solution for higher span-ratios. Thus, CLPT is an adequate analysis for "thin" laminates, although the concept of thickness for orthotropic materials depends not only on geometric ratios of the plate, but on differences in elastic properties of the laminae [10]. Evidence is given from Pagano [10], as shown in Figure 2.1; for a plate span-ratio of 10, the central plate deflection as given by classical plate theory ("CPT") is within approximately $12 \%$ of that given by an exact elasticity solution. Likewise, for a $\left[0^{\circ} / 90^{\circ} / 0^{\circ}\right]_{T}$ laminate ("3-LAYER COMPOSITE $(a=b) ")$ with the same span-ratio of 10 , the central plate deflection from classical plate theory is within $55 \%$ of that given by an exact elasticity solution. (The third line,

\footnotetext{
${ }^{3}$ Anisotropic plates was published in 1968.

${ }^{4}$ Dong et al. note: "Since the completion of this manuscript, a recent book by Ambartsumyan [3] has appeared. The general theory of anisotropic laminated shells, derived in a slightly different manner, is contained in Chapter II."

${ }^{5}$ Srinivas and Rao [7] developed solutions for a similar problem at approximately the same time as Pagano, though published later the same year. Some authors have additionally cited Vlasov [8] as solving the problem 13 years early, though an English translation of this publication could not be found. Unlike Srinivas and Rao, Pagano's work includes a comparison of the calculated transverse stresses from the elasticity solution to those found by applying the geometrically linear stress recovery procedure to results from a plate analysis.
} 
marked "SW PLATE" references a sandwich plate; though not of interest here, it serves to reinforce the preceding argument.)

Pagano's work $[9,10]$ is also important in that it is one of the first to compare the results of the transverse stresses obtained directly by the theory of elasticity to those obtained by integrating the equilibrium equations using the in-plane stresses from plate theory. It is interesting to note that Pagano does not show a comparison between the transverse normal stress as predicted by the exact and CLPT solutions, merely stating that CLPT (and using the equations of equilibrium) predicts this stress quite accurately [10]. Also noteworthy is the fact that both in-plane and transverse stresses given by CLPT converge more rapidly to the exact solution than the plate deflections [9-11], as errors for stresses are within $10 \%$ for laminates with span-to-thickness ratios as low as 20, while errors in displacements are greater than $10 \%$ for span-to-thickness ratios of at least 30 .

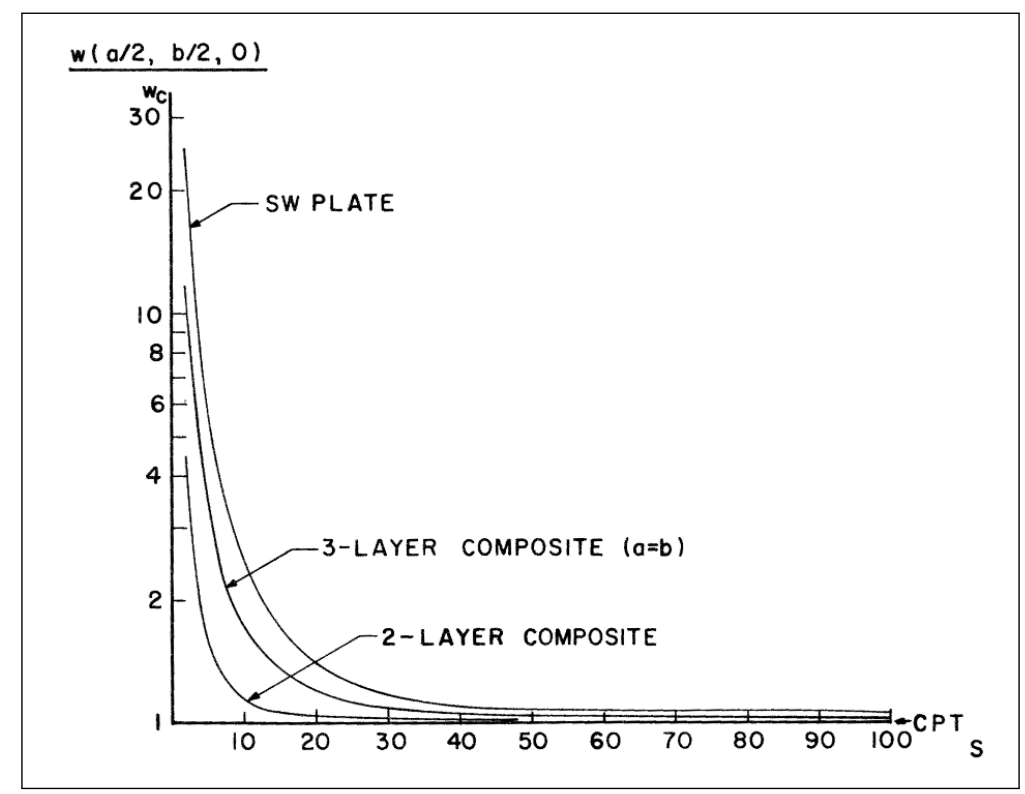

Figure 2.1: Central plate deflection given by classical plate theory ("CPT") and an exact elasticity solution with varying span-ratios. (From Pagano [10], Figure 2; used by permission; see Appendix D.1.) 


\subsubsection{Shear-Deformable Plate Theory}

Indeed, central to CLPT is the Kirchhoff hypothesis ${ }^{6}$, which results in the transverse strains being zero. In order to improve upon this assumption, Reissner [13], in 1945, developed a plate theory which accounted for the transverse shear effects through variational principles. Reissner's work was extended by Hildebrand, Reissner, and Thomas [14] in 1949 to curved shells. In 1951, Mindlin [15] derived the results of Reissner [13] through directly integrating the equations of equilibrium (rather than through variational methods). Additionally, Mindlin identified the relationship between the shear correction factor and Poisson's ratio of the material. Girkmann and Beer [16] subsequently extended Reissner's theory to orthotropic plates. According to Jones [11], the generalization to a laminated structure in which the principal material directions of the laminae align with the plate axes was made by Ambartsumyan [3]. Whitney [17] modified, then generalized, Ambartsumyan's work to symmetric laminates whose orthotropic layers have arbitrary orientations. Yang et al. [18] developed a Mindlin-type theory for laminated anisotropic plates including inertia terms, which Whitney and Pagano [19] modified.

Ashton and Whitney [20] compare the distribution of transverse shear stress as calculated by Whitney's shear-deformable theory to that given by the theory of elasticity [9]. Additionally, they calculate the transverse shear stress using the equilibrium equations of elasticity (with in-plane results from the shear-deformable theory). The three solutions (via the theory of elasticity, via Whitney's shear-deformable theory ${ }^{7}$ and that obtained by integrating equilibrium equations using in-plane data from Whitney's shear-deformable theory) are superimposed by Jones [11], as shown in Figure 2.2. The shear stress as given by the theory of elasticity (via Pagano [9]) is labeled "ELASTICITY SOLUTION," that given by Whit-

\footnotetext{
${ }^{6}$ Several authors use the term "Kirchhoff-Love hypothesis" or "Love-Kirchhoff hypothesis." Here the hypothesis of normals remaining straight, normal to the deformed mid-surface, and inextensible is simply referred to as the "Kirchhoff" hypothesis, though Love's [12] contribution is recognized.

${ }^{7}$ Whitney's shear-deformable solution, labeled as "SHEAR-DEFORMATION THEORY," is found by applying the transverse shear stress results from Whitney's shear-deformable plate theory and assuming a piecewise parabolic distribution through the plate thickness.
} 
ney's shear-deformable theory (via constitutive relations) is marked "SHEAR-DEFORMATION THEORY," and that resulting from use of the equilibrium equations is "MODIFIED SHEARDEFORMATION THEORY." Jones notes the excellent agreement between results from the "MODIFIED-SHEAR DEFORMATION THEORY" and "ELASTICITY SOLUTION."

\subsubsection{Higher-Order Theories}

The shear-deformable theories mentioned above are often referred to as first-order shear deformation theories; the in-plane displacements are first-order functions of the laminate thickness coordinate and, therefore, the transverse shear strains are constant through the laminate thickness. Thus, these theories do not allow for transverse shear stresses to vanish on the bounding surfaces of the plate, and require a shear-correction factor to account for the discrepancy [21, 22]. Third-order theories allow a parabolic distribution of transverse

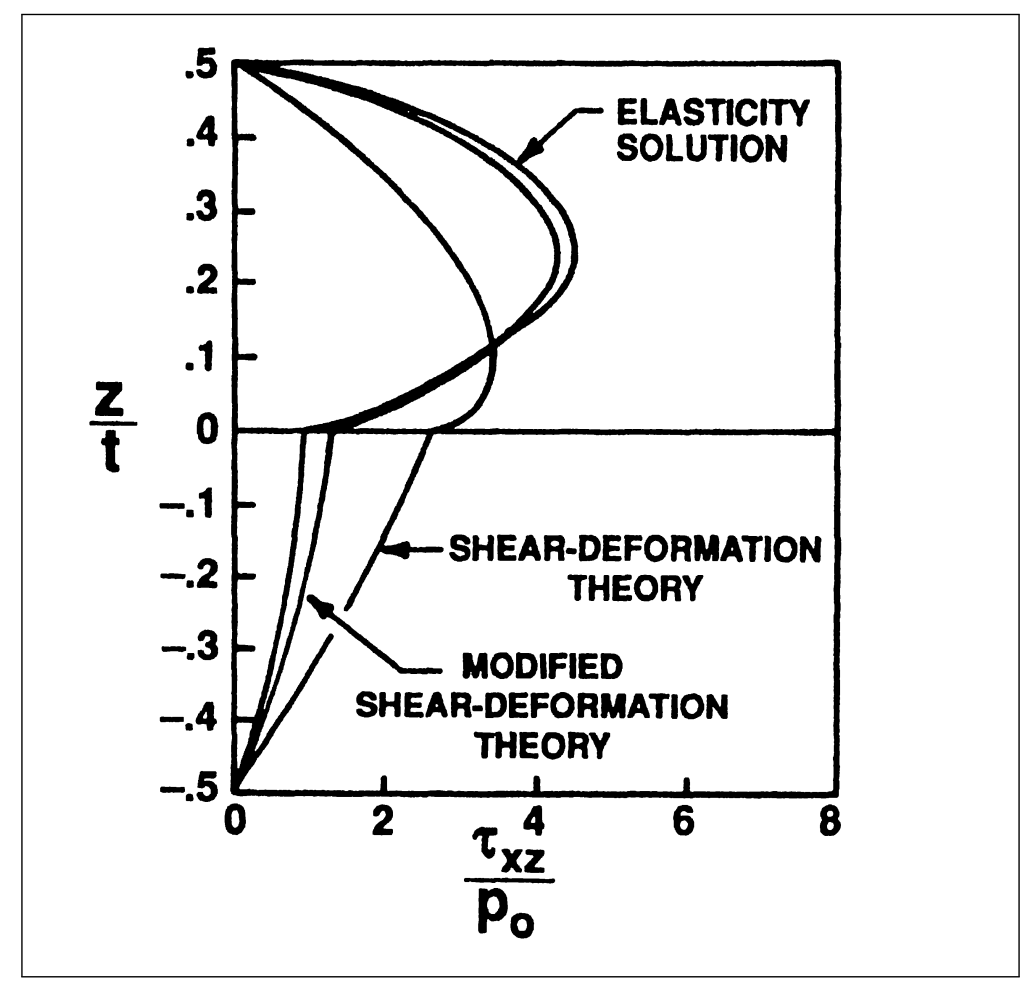

Figure 2.2: Transverse shear stress distribution along edge of semi-infinite strip. (From Jones [11], Figure 6-23; used by permission; see Appendix D.2.) 
shear stresses through the laminate thickness. Reddy [21] reviewed many third-order plate theories and concluded that all variationally derived theories are special cases of his 1984 publication [23]. Reddy [22] also noted that theories higher than third-order are generally not used, as they give little additional accuracy, particularly considering the disproportionate increase in computational effort.

The laminated plate theories discussed thus far may all be termed equivalent single layer ESL theories [22]. That is, a layered inhomogeneous plate is represented by an equivalent homogeneous plate; equivalent in the sense that the force and moment resultants at a cross section obtained by the integration of tractions through the thickness are identical. The assumption of a perfect bond between layers is then a necessity to these theories. Additionally, the kinematics of the equivalent homogeneous plate are defined such that transverse strains are continuous through the thickness. Because the material properties are discontinuous in the laminate, transverse stresses will not be continuous through the thickness, a result which does not satisfy Newton's third law.

\subsubsection{Layerwise Theories}

To account for the discrepancy of discontinuous transverse stresses, layerwise (LW) theories [24] describe the kinematics of individual laminae within a laminate. The continuous (through-the-thickness) piecewise assumed displacement functions may result in discontinuous strains which, because of the discontinuous layer properties, may result in continuous interlaminar stresses. Reddy has demonstrated the accuracy of such methods and presents [24] stress distributions through the thickness of a $\left[0^{\circ} / 90^{\circ} / 0^{\circ}\right]_{T}$ laminate subjected to a sinusoidally distributed transverse load. For in-plane and transverse stresses, the stresses given by Reddy's layerwise finite-element method virtually overlay Pagano's [10] elasticity solution for a thick plate (span-to-thickness ratio of 4 ).

However, because the LW methods employ nearly full three-dimensional kinematics, the efficiency of such methods may be on the order of that of a conventional three-dimensional 
finite-element solution. Additionally, LW finite elements are susceptible to hourglassing-type phenomena, particularly when modeling thin laminates. [24]

Carrera [25] considers layer-wise mixed theories to calculate interlaminar stresses. An overview of the state of equivalent single-layer (ESL) and layer-wise (LW) models is given. The author then proposes a layerwise approach using a mixed variational formulation based on Reissner's variational principle. Results are given for thick (span-to-thickness ratios greater than 4), square, cross-ply plates and compared with elasticity solutions (by Pagano), and other ESL and LW solutions. Mixed formulations in Carrera's work (with assumed parabolic forms of in-plane displacement variation through the thickness) give results in close agreement to the elasticity solution and better than work by references using other equivalent single-layer or layerwise solutions. In particular, the interlaminar stresses given by Carrera appear much closer to elasticity solutions than those of previous works. Carrera interestingly notes that the transverse shear stresses may be evaluated through integration of the equilibrium equations, but that results are inaccurate, particularly in thick plates. Furthermore, Carrera asserts that the procedure cannot be implemented in the general case of asymmetric laminates or nonlinear analyses.

\subsection{Determination of Interlaminar Stresses}

Many authors agree that the determination of interlaminar stresses in a composite laminate is an important part of laminate analysis; Pagano [9, 26], Jones [11], Hyer [27], and Puppo and Evensen [28] all note the importance of determining interlaminar stresses. Not only are the transverse strengths of composites typically much less than the in-plane strengths [27], but the interfaces of a laminate tend to be matrix-rich as well, adding to the inherent weakness of the interface. Consequently, much effort has been placed on accurately determining the distribution of interlaminar stresses in composite laminates. Indeed, a motivation for the higher-order plate theories discussed previously is a need for more accurate representations 
of interlaminar stresses than those given by CLPT, as the plane-stress assumption of CLPT precludes accurate calculation of these stresses. [9]

\subsubsection{Constitutive Equations}

The most direct method of determining the transverse stresses, for both analytical and numerical methods, is to apply the constitutive equations to the solution for the displacement field, solving directly for the stresses. However, except for three-dimensional or quasi-threedimensional theories, the stress field given by this method is far from accurate. This is not surprising, as the displacements through the thickness in reduced theories are approximations and not truly valid. For example, the transverse shear and normal strains within the framework of the classical plate theory are zero through the entire plate thickness. Using the constitutive law to calculate the transverse stresses from these strains yields transverse stresses that are identically zero. The cost of this inaccuracy is offset by the computational efficiency of the model. This is not implying that the constitutive law is flawed. Rather, calculating the stress field using the strain field and a constitutive law yields poor results for the transverse stresses, with the error being in the assumptions of the theory.

Similar to the classical plate theory, the first-order shear deformation theory yields, via constitutive equations, constant transverse shear stresses and zero transverse normal stress through the thickness. Not until the third-order are plate theories capable of predicting reasonable representations of the transverse stresses from constitutive equations. Yet even these are unable to represent laminates, in which the material properties may change drastically from layer to layer. The layerwise theories are able to account for the piecewise properties through a laminate thickness, though these are seen to be nearly as computationally onerous as full three-dimensional models. 


\subsubsection{Equilibrium Considerations-Stress Recovery}

The shortcomings of calculating the transverse stress field directly from constitutive equations may be overcome by use of the equations of equilibrium. Given an accurate representation of the in-plane stress state, the three equilibrium equations may be integrated through the plate thickness to determine the remaining three (transverse) stresses. Advantages include a fairly accurate representation of the distribution of transverse stresses, even for low-order plate theories, and fulfillment of the continuity requirement in tractions between layers (which cannot be realized using constitutive equations with any equivalent single layer theory). The accuracy of the equilibrium method depends on the accuracy of the given in-plane stresses and, in particular, their spatial gradients.

\subsubsection{Stress Recovery for Geometrically Linear Problems}

In the context of infinitesimal deformations, the stress recovery procedure uses the equations of motion for infinitesimal deformations. In a Cartesian coordinate system $x-y$ - $z$, in the absence of body forces and ignoring inertial effects, the equilibrium equations for infinitesimal deformations are given as

$$
\begin{aligned}
& \frac{\partial \sigma_{x x}}{\partial x}+\frac{\partial \tau_{x y}}{\partial y}+\frac{\partial \tau_{x z}}{\partial z}=0 \\
& \frac{\partial \tau_{x y}}{\partial x}+\frac{\partial \sigma_{y y}}{\partial y}+\frac{\partial \tau_{y z}}{\partial z}=0 \\
& \frac{\partial \tau_{x z}}{\partial x}+\frac{\partial \tau_{y z}}{\partial y}+\frac{\partial \sigma_{z z}}{\partial z}=0
\end{aligned}
$$

in which the stresses $\sigma_{x x}, \sigma_{y y}, \sigma_{z z}, \tau_{y z}, \tau_{x z}$, and $\tau_{x y}$ are the Cauchy stresses oriented in the reference coordinate system and differentiation is performed in the reference coordinate system. These three equations, while formally constituting a set of linear first-order partial differential equations involving the stresses, can be considered as three linear algebraic equations in the stress gradients. Indeed, the gradient of one stress in a particular coordinate 
direction causes there to be gradients in the other directions involving other stresses. The above three equations can be integrated through the thickness ( $z$-direction) of the plate so the transverse stresses $\left(\tau_{y z}, \tau_{x z}, \sigma_{z z}\right)$ are functions of the in-plane stresses $\left(\sigma_{x x}, \sigma_{y y}, \tau_{x y}\right)$ :

$$
\begin{aligned}
\tau_{x z} & =-\int_{z_{0}}^{z}\left(\frac{\partial \sigma_{x x}}{\partial x}+\frac{\partial \tau_{x y}}{\partial y}\right) \mathrm{d} z+\tau_{x z}^{0}(x, y) \\
\tau_{y z} & =-\int_{z_{0}}^{z}\left(\frac{\partial \tau_{x y}}{\partial x}+\frac{\partial \sigma_{y y}}{\partial y}\right) \mathrm{d} z+\tau_{y z}^{0}(x, y) \\
\sigma_{z z} & =-\int_{z_{0}}^{z}\left(\frac{\partial \tau_{x z}}{\partial x}+\frac{\partial \tau_{y z}}{\partial y}\right) \mathrm{d} z+\sigma_{z z}^{0}(x, y)
\end{aligned}
$$

in which a superscript naught indicates the value of a stress at a reference position, typically the upper or lower plate surface. In this way, the transverse stresses $\left(\tau_{y z}, \tau_{x z}, \sigma_{z z}\right)$, though initially perhaps neglected, are recovered from the known in-plane stresses $\left(\sigma_{x x}, \sigma_{y y}, \tau_{x y}\right)$. This is the basis for the procedure being commonly termed stress recovery.

Pagano $[9,10]$ compared transverse stresses calculated from Eq. (2.2) to exact solutions from the linear theory of elasticity and found good agreement, providing the plate spanto-thickness ratio (referred to simply as span-ratio from here forward) is sufficiently large (i.e., the Kirchhoff hypothesis is sufficiently valid). Nearly every equivalent single layer plate theory uses the equilibrium method to determine the transverse stresses.

Pryor and Barker [29] implement Whitney's [17] shear-deformable theory in a finite element formulation, integrating equilibrium equations to calculate the transverse shear stresses. Transverse normal stress is not mentioned. According to Pryor and Barker [29], "the thickness concept, as it is known for isotropic plates, is different for heterogeneous anisotropic plates. For such plates, the distortion of the deformed normal due to transverse shear is dependent, not only on the laminate thickness, but also on the orientation and degree of orthotropy of the individual layers [29]." Engblom and Ochoa [30] integrate the equation of equilibrium in the transverse direction to find the transverse normal stress.

Bhaskar and Varadan [31] use a shear-deformable theory to find a closed-form (but approximate) solution to the intralaminar stresses for an infinitely long, simply-supported, 
cylindrical shell. Interlaminar stresses are then found by integrating the linear, dynamic equations of equilibrium through the thickness of the shell. Their goal is to determine the dynamic magnification factors (the ratio of the maximum dynamic response to the corresponding static response) in cylindrical shells under sinusoidal loading. Results are compared to the exact solution by Varadan and Bhaskar [32]. The authors apply their formulation to the cases of a sinusoidal load and a centrally located uniform load, both loads considering a step and rectangular pulse function in the time domain.

\subsubsection{Stress Recovery for Geometrically Nonlinear Problems}

For problems in which the strain-displacement relations are nonlinear, the equilibrium equations of Eq. (2.1) cease to apply. If strains are small compared to unity and rotations of body elements are small (though finite) compared to unity, the equilibrium equations are given instead by

$$
\begin{aligned}
\frac{\partial}{\partial x}\left(\sigma_{x x}-\omega_{z} \tau_{x y}+\omega_{y} \tau_{x z}\right) & +\frac{\partial}{\partial y}\left(\tau_{x y}-\omega_{z} \sigma_{y y}+\omega_{y} \tau_{y z}\right) \\
& +\frac{\partial}{\partial z}\left(\tau_{x z}-\omega_{z} \tau_{y z}+\omega_{y} \sigma_{z z}\right)+F_{X}^{*}=0 \\
\frac{\partial}{\partial x}\left(\omega_{z} \sigma_{x x}+\tau_{x y}-\omega_{x} \tau_{x z}\right) & +\frac{\partial}{\partial y}\left(\omega_{z} \tau_{x y}+\sigma_{y y}-\omega_{x} \tau_{y z}\right) \\
& +\frac{\partial}{\partial z}\left(\omega_{z} \tau_{x z}+\tau_{y z}-\omega_{x} \sigma_{z z}\right)+F_{Y}^{*}=0 \\
\frac{\partial}{\partial x}\left(-\omega_{y} \sigma_{x x}+\omega_{x} \tau_{x y}+\tau_{x z}\right) & +\frac{\partial}{\partial y}\left(-\omega_{y} \tau_{x y}+\omega_{x} \sigma_{y y}+\tau_{y z}\right) \\
& +\frac{\partial}{\partial z}\left(-\omega_{y} \tau_{x z}+\omega_{x} \tau_{y z}+\sigma_{z z}\right)+F_{Z}^{*}=0
\end{aligned}
$$

in which $\omega_{x}, \omega_{y}$, and $\omega_{z}$ represent the rotations about the axes of the undeformed global coordinate system, and $F_{X}^{*}, F_{Y}^{*}$, and $F_{Z}^{*}$ represent body force effects, including inertial effects in the sense of D'Alembert, aligning with the axes of the undeformed global coordinate system. Because the rotations $\omega_{x}, \omega_{y}$, and $\omega_{z}$ are small but finite, rather than infinitesimal, they are commonly termed moderate rotations. 
Reddy [24] notes that the partial differential equations that govern the behavior of laminated composite plates cannot, in general, be solved exactly. While some exact elasticity solutions considering simple geometries and boundary and loading conditions are available, those including geometric nonlinearities do not yet exist. Indeed, the governing equations that must be solved become complicated quickly, as evidenced by the progression from Eq. (2.1) to Eq. (2.3).

Barbero and Reddy [33] employ a geometrically nonlinear finite element formulation which includes the von Kármán nonlinearity (moderate rotations) in the kinematics. Results are presented in the nonlinear regime, but the transverse normal stress is not shown and transverse shear stresses are not compared to those from a three-dimensional solution. Further, the equilibrium equations which are integrated to find the transverse stresses neglect the nonlinear terms.

Byun and Kapania [34] formulate a post-processing procedure which retains some of the geometrically nonlinear terms of the equilibrium equations for the transverse normal stress. Rotations about the transverse plate axis, $\omega_{z}$, are neglected. Terms in which transverse stresses are multiplied by rotations are neglected as well. Thus, using the authors' notation, equilibrium equations, for the $k^{\text {th }}$ layer, are stated as

$$
\begin{array}{r}
\sigma_{x, x}^{k}+\tau_{x y, y}^{k}+\tau_{x z, z}^{k}=0 \\
\tau_{x y, x}^{k}+\sigma_{y, y}^{k}+\tau_{y z, z}^{k}=0 \\
\tau_{x z, x}^{k}+\tau_{y z, y}^{k}+\sigma_{z, z}^{k}+\sigma_{x}^{k} w_{, x x}+2 \tau_{x y}^{k} w_{, x y}+\sigma_{y}^{k} w_{, y y}=0
\end{array}
$$

in which a comma in the subscript denotes differentiation, and the last three terms of Eq. (2.4c) (those containing the transverse deflection $w$ ) are the nonlinear terms. Only numerical results for small-deflection (i.e. geometrically linear) solutions are presented by Byun and Kapania [34] (albeit for rather thick plates with span-to-thickness ratios of 4).

Park et al. [35] mentioned Reissner [36] and Al-Qarra [37] as authors noting the impor- 
tance of geometric nonlinearities in the analysis of laminated composites. They indicated that few papers they had seen account for geometric nonlinearities. Park et al. proceed in describing their procedure, which is based upon their work in predictor-corrector methods (Park and Kim [38, 39]), but incorporates the effects of geometrical nonlinearities. The base finite element model contains two unknowns, displacements and strains which have independently assumed forms. The post-processing procedure consists of the following steps:

1. Obtained data from a mixed plate finite element formulation.

2. Calculate transverse stresses from linear equilibrium equations.

3. Calculate transverse strains from initial transverse stresses through constitutive relations.

4. Calculate displacements from strains.

5. Correct displacements through matching averages and slopes with those from the initial finite element analysis from Step 1.

6. Recalculate displacements.

7. Calculate transverse strains through strain-displacement relations.

8. Iterate steps $4-7$ until convergence criterion is satisfied.

9. Integrate nonlinear equilibrium equations with updated displacements and stresses.

Results are shown for a composite panel (span-ratio of 100) and sandwich plate (span-ratio of 20) and compared to results from a three-dimensional finite element analysis. Transverse stress distributions compare well to distributions from their benchmark three-dimensional finite element solution. 


\subsubsection{Numerical Methods}

Several authors have published methods to determine interlaminar stresses from reducedorder models that are similar to the stress recovery procedure. Those cited here employ the finite element method either as a post-processing routine to recover the transverse stresses, or by developing custom finite element formulations.

Chaudhuri and Seide [40] use a previously developed triangular finite element [41] and recover interlaminar shear stresses by forcing the transverse shear stresses to vanish at the bounding surfaces and satisfying continuity of the transverse stresses at the interfaces. They use a layerwise constant shear angle theory and match interface stresses through the linear equilibrium equations. They emphasize the ability of their method to find accurate transverse stresses for asymmetrically laminated plates.

Lee and Lee [42] formulated a post-processing procedure for determining transverse stresses in composite and sandwich structures undergoing finite rotations. The approach uses displacements and stresses as calculated by a plate finite element analysis. A point of interest is considered in the plate (at an element centroid) and an additional finite element analysis is performed at that location through the thickness of the plate using onedimensional elements. The formulation for these elements uses the geometrically nonlinear equilibrium equations. Thus, the equations of equilibrium are not directly integrated, but are used in the formulation of a finite element through the thickness of the plate.

To obtain the necessary gradients of stresses, the authors fit polynomials to stress values at several points in the $x$ - and $y$-directions. They note that other methods (in particular the super-convergent recovery technique [43]) can be used, inputting the results into their post-processing finite element analysis.

Lee and Lee [42] compare solutions of several examples problems using their technique to analytical or three-dimensional finite element solutions of these problems: a cantilever beam, a clamped composite plate, and a clamped sandwich plate. For geometrically linear 
solutions, good agreement is shown for the transverse shear stresses, but their model and the results from a three-dimensional finite element solution differ in the transverse normal stress. For geometrically nonlinear solutions, no three-dimensional analysis is presented for comparison.

Lee et al. [44] extended the work of Lee and Lee [42] to include inertia effects. Panels with span-ratios of 64 and 128 are evaluated under the influence of sinusoidal and triangular time-varying pressure loads. No comparison is made to a three-dimensional solution.

Dakshina Moorthy and Reddy [45] use a layerwise displacement finite element with enhanced strains to determine deflections of a thick laminated composite plate. They add an interface model using the penalty method to determine the interlaminar stresses, strains, and strain energy release rates at a delamination. These methods, they note, work well for thick plates, but tend to behave erratically for thinner plates (span-to-thickness ratios above 40). The layerwise finite elements are used for each sub-laminate, that is, a location for delamination is chosen a priori, the resulting separate layers (sub-laminates) are then modeled with the layer-wise elements. The sub-laminates are joined through adhesive contact, modeled with the penalty method. From these contact forces, the tractions at the delamination are calculated.

Fagiano et al. [46] follow Dakshina Moorthy and Reddy [45] by modeling each lamina of a laminate as a discrete body of three-dimensional finite elements. They then extend that work by enforcing compatibility between layers using Lagrange multipliers (cf. the penalty method [45]), and the resulting traction on the interlaminar boundary is interpolated over the entire surface (cf. per element group [45]). The authors then obtain more accurate interlaminar stresses from a three-dimensional analysis, as they note that "three-dimensional stress states cannot, in general, be accurately analysed" with plate theory. Stresses are found around the free-edge of a central hole in a three-layer cross-ply rectangular laminate (the $\left[0^{\circ} / 90^{\circ} / 0^{\circ}\right]_{T}$ used by Pagano $\left.[9,10]\right)$. The authors build upon the work in a second paper $[47]$ by automating the stress recovery routine to: 
1. Perform a three-dimensional analysis using Abaqus.

2. Extract model stress and displacement data using Abaqus' Python API.

3. Do stress recovery in MATLAB.

4. Insert recovered stresses in Abaqus database file using Abaqus' Python API.

5. View results in Abaqus CAE.

They validate the routine on the same rectangular plate as in their first paper, then use the implementation to solve for interlaminar stresses in a simply-supported cylindrical shell, comparing results to the exact solution by Varadan and Bhaskar [32].

\subsection{Summary of Considerations for Geometric Nonlinearities}

Few authors have considered the accurate estimation of transverse stresses of laminates undergoing large deflections. Chia [48] notes the importance of including rotation terms in calculating stresses and strains, especially for thin plates. The influence of rotations about in-plane axes overwhelm those of rotations about a perpendicular axis, and Chia neglects the latter for kinematic relations and equilibrium equations. Libai and Simmonds [49] mention, briefly, that one may estimate transverse shear stresses by "integrating the differential equations of equilibrium in the thickness direction" but do not show it and no discussion is presented as to whether the nonlinear equations must be used or the linear equations would suffice.

Papers by Barbero and Reddy [33], Engelstad et al. [50], Byun and Kapania [34], Park

et al. [35], Lee and Lee [42], and Lee et al. [44] have included geometrically nonlinear effects to some extent, although the procedures can become complex and, perhaps, difficult to implement. Furthermore, these are all carried out in the finite element method. Of those 
mentioned, Byun and Kapania [34] included some nonlinear terms, but not others, in the equilibrium equation for the transverse direction. No mention is made as to why some are omitted. Park et al. [35] apply the integration of simplified nonlinear equilibrium equations as a last step in a predictor-corrector method, after the displacement distributions have been corrected from a plate finite element analysis. Lee et al. [44] employ an additional finite element analysis (after the finite element analysis used to find the in-plane response) to solve the nonlinear equations of equilibrium, including inertial effects. Results of transverse stresses are presented (though only as time-histories), but are not compared to any other solution (such as a three-dimensional finite element solution). 


\section{Chapter 3}

\section{Geometrically Nonlinear}

\section{Stress Recovery}

The geometrically nonlinear stress recovery procedure is now developed for application to flat plates. The application of the procedure to recover stresses from a discretized solution, such as a finite element solution, is discussed. The method by which the accuracy of the procedure will be evaluated in subsequent chapters is also developed.

\subsection{Geometrically Nonlinear Equilibrium Equations}

Consider the undeformed flat plate shown in Figure 3.1a. A global coordinate system $X-Y-Z$ is positioned at the geometric center of the plate, with the $X-Y$ plane coincident with the plate geometric midsurface, generally taken as the plate reference surface. Additionally, a body-centered coordinate system $x-y-z$ coincides with the global coordinate system in the undeformed configuration. The deformed configuration of the plate is shown in Figure 3.1b. The global coordinate system is unchanged, but the body-centered coordinate system, originally Cartesian, is now curvilinear.

From Novozhilov [51], the equilibrium equations for a geometrically nonlinear analysis considering small strains and moderate (in the sense of von Kármán) rotations are given by 


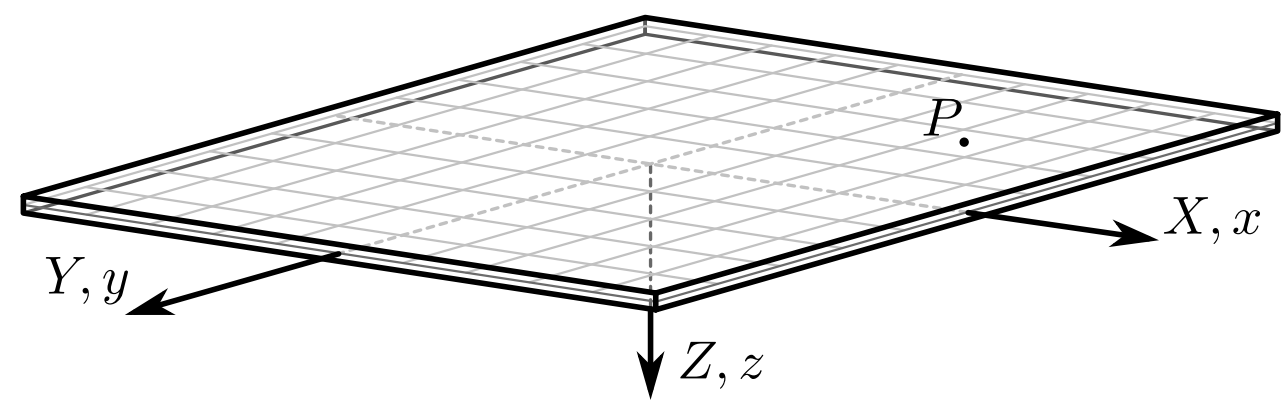

(a) Undeformed configuration.

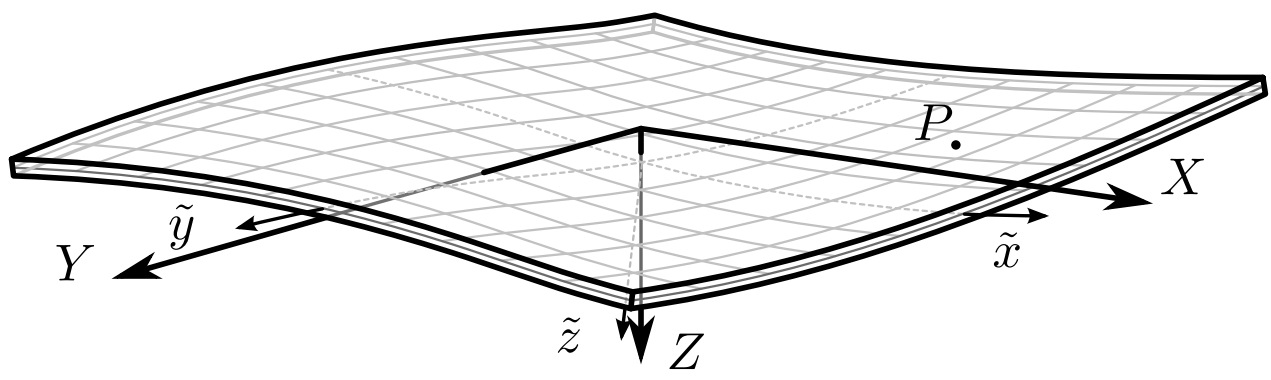

(b) Deformed configuration.

Figure 3.1: Coordinate system definitions.

$$
\begin{aligned}
\frac{\partial}{\partial x}\left(\sigma_{x x}-\omega_{z} \tau_{x y}+\omega_{y} \tau_{x z}\right) & +\frac{\partial}{\partial y}\left(\tau_{x y}-\omega_{z} \sigma_{y y}+\omega_{y} \tau_{y z}\right) \\
& +\frac{\partial}{\partial z}\left(\tau_{x z}-\omega_{z} \tau_{y z}+\omega_{y} \sigma_{z z}\right)+F_{X}^{*}=0 \\
\frac{\partial}{\partial x}\left(\omega_{z} \sigma_{x x}+\tau_{x y}-\omega_{x} \tau_{x z}\right) & +\frac{\partial}{\partial y}\left(\omega_{z} \tau_{x y}+\sigma_{y y}-\omega_{x} \tau_{y z}\right) \\
& +\frac{\partial}{\partial z}\left(\omega_{z} \tau_{x z}+\tau_{y z}-\omega_{x} \sigma_{z z}\right)+F_{Y}^{*}=0 \\
\frac{\partial}{\partial x}\left(-\omega_{y} \sigma_{x x}+\omega_{x} \tau_{x y}+\tau_{x z}\right) & +\frac{\partial}{\partial y}\left(-\omega_{y} \tau_{x y}+\omega_{x} \sigma_{y y}+\tau_{y z}\right) \\
& +\frac{\partial}{\partial z}\left(-\omega_{y} \tau_{x z}+\omega_{x} \tau_{y z}+\sigma_{z z}\right)+F_{Z}^{*}=0
\end{aligned}
$$

where the stress components are given in the body-centered $x-y-z$ coordinate system, and 
the terms $\omega_{x}, \omega_{y}$, and $\omega_{z}$ are given by

$$
\begin{aligned}
\omega_{x} & =\frac{1}{2}\left(\frac{\partial w}{\partial y}-\frac{\partial v}{\partial z}\right) \\
\omega_{y} & =\frac{1}{2}\left(\frac{\partial u}{\partial z}-\frac{\partial w}{\partial x}\right) \\
\omega_{z} & =\frac{1}{2}\left(\frac{\partial v}{\partial x}-\frac{\partial u}{\partial y}\right)
\end{aligned}
$$

which, for moderate rotations, can be considered the rotations about the global $X, Y$, and $Z$ axes, respectively [51]. For the derivation of Eq. (3.1) from the equations of equilibrium from the nonlinear theory of elasticity, the reader is directed to Appendix A, where Eq. (3.1) is given by Eq. (A.13).

If the only body forces that exist are those arising due to non-zero accelerations in the inertial terms, then, by D'Alembert's principle,

$$
\begin{aligned}
& F_{X}^{*}=-\rho a_{X} \\
& F_{Y}^{*}=-\rho a_{Y} \\
& F_{Z}^{*}=-\rho a_{Z}
\end{aligned}
$$

However, to keep the general applicability of the stress recovery procedure, the body force terms will retain the notation found in Eq. (3.1). The relations of Eq. (3.3) may then be applied at any point in the outline that follows.

\subsection{The Stress Recovery Procedure}

With the equilibrium equations of Eq. (3.1), the geometrically nonlinear stress recovery procedure begins by integrating each equation with respect to $z$. For Eq. (3.1a), this integration 
gives

$$
\begin{aligned}
\left(\tau_{x z}-\right. & \left.\omega_{z} \tau_{y z}+\omega_{y} \sigma_{z z}\right)\left.\right|_{z^{0}} ^{z}= \\
& -\int_{z^{0}}^{z}\left(\frac{\partial}{\partial x}\left(\sigma_{x x}-\omega_{z} \tau_{x y}+\omega_{y} \tau_{x z}\right)+\frac{\partial}{\partial y}\left(\tau_{x y}-\omega_{z} \sigma_{y y}+\omega_{y} \tau_{y z}\right)+F_{X}^{*}\right) \mathrm{d} z
\end{aligned}
$$

where the terminology $\left.f\right|_{z^{0}} ^{z}$ indicates the expression $f$ evaluated at $z_{0}$ is to be subtracted from the expression $f$ evaluated at an arbitrary location $z$ within the thickness of the plate. Likewise, the remaining equilibrium equations Eqs. (3.1b) and (3.1c) are integrated:

$$
\begin{aligned}
\left(\omega_{z} \tau_{x z}\right. & \left.+\tau_{y z}-\omega_{x} \sigma_{z z}\right)\left.\right|_{z^{0}} ^{z}= \\
& -\int_{z^{0}}^{z}\left(\frac{\partial}{\partial x}\left(\omega_{z} \sigma_{x x}+\tau_{x y}-\omega_{x} \tau_{x z}\right)+\frac{\partial}{\partial y}\left(\omega_{z} \tau_{x y}+\sigma_{y y}-\omega_{x} \tau_{y z}\right)+F_{Y}^{*}\right) \mathrm{d} z \\
\left(-\omega_{y} \tau_{x z}\right. & \left.+\omega_{x} \tau_{y z}+\sigma_{z z}\right)\left.\right|_{z^{0}} ^{z}= \\
& -\int_{z^{0}}^{z}\left(\frac{\partial}{\partial x}\left(-\omega_{y} \sigma_{x x}+\omega_{x} \tau_{x y}+\tau_{x z}\right)+\frac{\partial}{\partial y}\left(-\omega_{y} \tau_{x y}+\omega_{x} \sigma_{y y}+\tau_{y z}\right)+F_{Z}^{*}\right) \mathrm{d} z
\end{aligned}
$$

The terms on the right hand sides of Eq. (3.4) are functions of the values and gradients of the rotations, the in-plane stress components, and the transverse shear stress components. Let the expressions on the right hand sides of Eq. (3.4) be given by functions $f_{x z}, f_{y z}$, and $f_{z z}$. Because the transverse stress components (namely $\tau_{x z}$ and $\tau_{y z}$ ) appear on both sides of Eq. (3.4), an iterative scheme is introduced whereby initial values of the transverse stress components $\tau_{x z}$ and $\tau_{y z}$ are used to calculate a first iteration of the left hand side transverse stress components. Let the iteration step be denoted by a parenthetic superscript such that the initial value of the transverse shear stress $\tau_{x z}$ is given by $\tau_{x z}^{(0)}$, while the first iteration of that stress is given by $\tau_{x z}^{(1)}$. With this notation, the $i^{\text {th }}$ iteration of the right hand sides of Eq. (3.4) are written as

$$
f_{x z}^{(i)}=-\int_{z^{0}}^{z}\left(\frac{\partial}{\partial x}\left(\sigma_{x x}-\omega_{z} \tau_{x y}+\omega_{y} \tau_{x z}^{(i)}\right)+\frac{\partial}{\partial y}\left(\tau_{x y}-\omega_{z} \sigma_{y y}+\omega_{y} \tau_{y z}^{(i)}\right)+F_{X}^{*}\right) \mathrm{d} z
$$




$$
\begin{aligned}
& f_{y z}^{(i)}=-\int_{z^{0}}^{z}\left(\frac{\partial}{\partial x}\left(\omega_{z} \sigma_{x x}+\tau_{x y}-\omega_{x} \tau_{x z}^{(i)}\right)+\frac{\partial}{\partial y}\left(\omega_{z} \tau_{x y}+\sigma_{y y}-\omega_{x} \tau_{y z}^{(i)}\right)+F_{Y}^{*}\right) \mathrm{d} z \\
& f_{z z}^{(i)}=-\int_{z^{0}}^{z}\left(\frac{\partial}{\partial x}\left(-\omega_{y} \sigma_{x x}+\omega_{x} \tau_{x y}+\tau_{x z}^{(i)}\right)+\frac{\partial}{\partial y}\left(-\omega_{y} \tau_{x y}+\omega_{x} \sigma_{y y}+\tau_{y z}^{(i)}\right)+F_{Z}^{*}\right) \mathrm{d} z
\end{aligned}
$$

For plate solutions in which the rotations are independent of the thickness coordinate, $z$, Eq. (3.4) now be rewritten as

$$
\begin{aligned}
\left(\tau_{x z}-\tau_{x z}^{0}\right)-\omega_{z}\left(\tau_{y z}-\tau_{y z}^{0}\right)+\omega_{y}\left(\sigma_{z z}-\sigma_{z z}^{0}\right) & =f_{x z} \\
\omega_{z}\left(\tau_{x z}-\tau_{x z}^{0}\right)+\left(\tau_{y z}-\tau_{y z}^{0}\right)-\omega_{x}\left(\sigma_{z z}-\sigma_{z z}^{0}\right) & =f_{y z} \\
-\omega_{y}\left(\tau_{x z}-\tau_{x z}^{0}\right)+\omega_{x}\left(\tau_{y z}-\tau_{y z}^{0}\right)+\quad\left(\sigma_{z z}-\sigma_{z z}^{0}\right) & =f_{z z}
\end{aligned}
$$

with the superscript zero indicating the value of that transverse stress component evaluated at the reference location $z=z_{0}$.

Equation (3.6) may be written more compactly in matrix form:

$$
\left(\begin{array}{ccc}
1 & -\omega_{z} & \omega_{y} \\
\omega_{z} & 1 & -\omega_{x} \\
-\omega_{y} & \omega_{x} & 1
\end{array}\right) \cdot\left\{\begin{array}{c}
\tau_{x z}-\tau_{x z}^{0} \\
\tau_{y z}-\tau_{y z}^{0} \\
\sigma_{z z}-\sigma_{z z}^{0}
\end{array}\right\}=\left\{\begin{array}{c}
f_{x z} \\
f_{y z} \\
f_{z z}
\end{array}\right\}
$$

Solving Eq. (3.7) for the transverse stresses $\tau_{x z}, \tau_{y z}$, and $\sigma_{z z}$ and adding notation to indicate the iteration step, the $i^{\text {th }}$ iteration of the recovered transverse stress components is given as

$$
\left\{\begin{array}{c}
\tau_{x z}^{(i)} \\
\tau_{y z}^{(i)} \\
\sigma_{z z}^{(i)}
\end{array}\right\}=\left(\begin{array}{ccc}
1 & -\omega_{z} & \omega_{y} \\
\omega_{z} & 1 & -\omega_{x} \\
-\omega_{y} & \omega_{x} & 1
\end{array}\right)^{-1}\left\{\begin{array}{l}
f_{x z}^{(i-1)} \\
f_{y z}^{(i-1)} \\
f_{z z}^{(i-1)}
\end{array}\right\}+\left\{\begin{array}{c}
\tau_{x z}^{0} \\
\tau_{y z}^{0} \\
\sigma_{z z}^{0}
\end{array}\right\}
$$


The inverse of the rotation matrix is given by

$$
\left(\begin{array}{ccc}
1 & -\omega_{z} & \omega_{y} \\
\omega_{z} & 1 & -\omega_{x} \\
-\omega_{y} & \omega_{x} & 1
\end{array}\right)^{-1}=\left(\begin{array}{ccc}
\frac{1+\omega_{x}^{2}}{1+\omega_{x}^{2}+\omega_{y}^{2}+\omega_{z}^{2}} & \frac{\omega_{x} \omega_{y}+\omega_{z}}{1+\omega_{x}^{2}+\omega_{y}^{2}+\omega_{z}^{2}} & \frac{-\omega_{y}+\omega_{x} \omega_{z}}{1+\omega_{x}^{2}+\omega_{y}^{2}+\omega_{z}^{2}} \\
\frac{\omega_{x} \omega_{y}-\omega_{z}}{1+\omega_{x}^{2}+\omega_{y}^{2}+\omega_{z}^{2}} & \frac{1+\omega_{y}^{2}}{1+\omega_{x}^{2}+\omega_{y}^{2}+\omega_{z}^{2}} & \frac{\omega_{x}+\omega_{y} \omega_{z}}{1+\omega_{x}^{2}+\omega_{y}^{2}+\omega_{z}^{2}} \\
\frac{\omega_{y}+\omega_{x} \omega_{z}}{1+\omega_{x}^{2}+\omega_{y}^{2}+\omega_{z}^{2}} & \frac{-\omega_{x}+\omega_{y} \omega_{z}}{1+\omega_{x}^{2}+\omega_{y}^{2}+\omega_{z}^{2}} & \frac{1+\omega_{z}^{2}}{1+\omega_{x}^{2}+\omega_{y}^{2}+\omega_{z}^{2}}
\end{array}\right)
$$

With rotations considered small compared to unity, Eq. (3.9) may be simplified as

$$
\left(\begin{array}{ccc}
1 & -\omega_{z} & \omega_{y} \\
\omega_{z} & 1 & -\omega_{x} \\
-\omega_{y} & \omega_{x} & 1
\end{array}\right)^{-1} \approx\left(\begin{array}{ccc}
1 & \omega_{z} & -\omega_{y} \\
-\omega_{z} & 1 & \omega_{x} \\
\omega_{y} & -\omega_{x} & 1
\end{array}\right)
$$

so that Eq. (3.8) becomes

$$
\left\{\begin{array}{c}
\tau_{x z}^{(i)} \\
\tau_{y z}^{(i)} \\
\sigma_{z z}^{(i)}
\end{array}\right\}=\left(\begin{array}{ccc}
1 & \omega_{z} & -\omega_{y} \\
-\omega_{z} & 1 & \omega_{x} \\
\omega_{y} & -\omega_{x} & 1
\end{array}\right)\left\{\begin{array}{l}
f_{x z}^{(i-1)} \\
f_{y z}^{(i-1)} \\
f_{z z}^{(i-1)}
\end{array}\right\}+\left\{\begin{array}{c}
\tau_{x z}^{0} \\
\tau_{y z}^{0} \\
\sigma_{z z}^{0}
\end{array}\right\}
$$

\subsection{Application to Laminated Plates}

Consider a plate, laminated through the thickness, as depicted in Figure 3.2, such that the outermost layer in the $+z$-direction is the first layer ${ }^{1}$, Layer \#1. The outermost location of Layer $\# 1$ is the bottom of the laminated plate, located at $z=z_{0}$, and the thickness of that layer is given by $t_{1}$. This continues through to the outermost layer in the $-z$-direction, Layer $\# N$, whose outermost location is the top of the laminated plate, located at $z=z_{N}$.

The recovered transverse stresses of Eq. (3.11) are calculated on a layer-by-layer basis

\footnotetext{
${ }^{1}$ The notation of the order of layers in the laminate of Figure 3.2 is reverse that of classical lamination theory. This is intentional, so that the recovery process starts from $j=1$ at the $+z$-surface, which is traction-free in the example problems, and proceeds to the $j=N$ at the $-z$-surface, where tractions are applied in the example problems. This arrangement is merely convenient for the example problems studied. Layer numbers and the integration process could just as easily start from the $-z$-surface.
} 


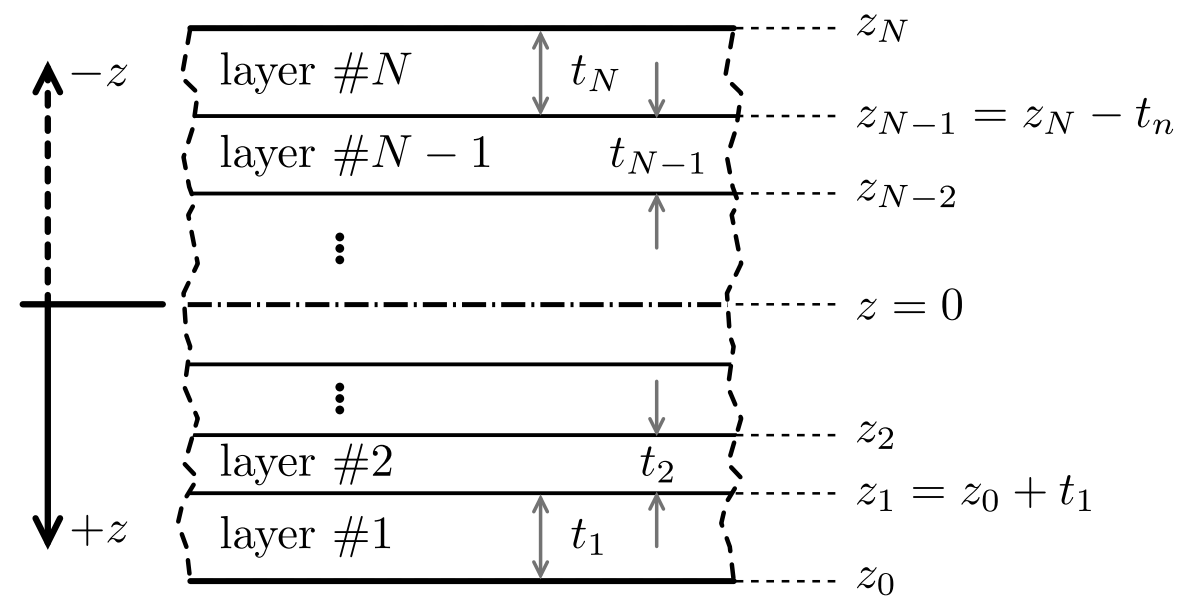

Figure 3.2: Notation of layer numbering and locations through the thickness of a laminated plate.

through the thickness of the laminate so that, for the $j^{\text {th }}$ layer, the reference stresses $\tau_{x z}^{0}$, $\tau_{y z}^{0}$, and $\sigma_{z z}^{0}$ are the recovered transverse stresses from the $(j-1)^{\text {th }}$ layer calculated at the interface of the two layers. For Layer $\# 1$, the values of $\tau_{x z}^{0}, \tau_{y z}^{0}$, and $\sigma_{z z}^{0}$ are the traction boundary conditions at the bottom surface. Note that the traction boundary condition for the top surface is not used in this method. Using a prefixed superscript to identify the layer number, Eq. (3.11) is rewritten as

$$
\left\{\begin{array}{c}
{ }^{j} \tau_{x z}^{(i)} \\
{ }^{j} \tau_{y z}^{(i)} \\
{ }^{j} \sigma_{z z}^{(i)}
\end{array}\right\}=\left(\begin{array}{ccc}
1 & \omega_{z} & -\omega_{y} \\
-\omega_{z} & 1 & \omega_{x} \\
\omega_{y} & -\omega_{x} & 1
\end{array}\right)\left\{\begin{array}{c}
j f_{x z}^{(i-1)} \\
j f_{y z}^{(i-1)} \\
j f_{z z}^{(i-1)}
\end{array}\right\}+\left\{\begin{array}{l}
\left.{ }^{j-1} \tau_{x z}^{0}\right|_{z=z_{j-1}} \\
j-\left.1 \tau_{y z}^{0}\right|_{z=z_{j-1}} \\
j-\left.1 \sigma_{z z}^{0}\right|_{z=z_{j-1}}
\end{array}\right\}
$$

With the rotations $\omega_{x}, \omega_{y}$, and $\omega_{z}$ given from the solution of a plate analysis, the intensive portion of the calculation of Eq. (3.12) is in determining the values of the integrals of Eq. (3.5) $\left({ }^{j} f_{x z},{ }^{j} f_{y z}\right.$, and $\left.{ }^{j} f_{z z}\right)$ in each layer. Recall again the integral functions of Eq. (3.5), repeated here (omitting the iteration notation), are

$$
f_{x z}=-\int_{z^{0}}^{z}\left(\frac{\partial}{\partial x}\left(\sigma_{x x}-\omega_{z} \tau_{x y}+\omega_{y} \tau_{x z}\right)+\frac{\partial}{\partial y}\left(\tau_{x y}-\omega_{z} \sigma_{y y}+\omega_{y} \tau_{y z}\right)+F_{X}^{*}\right) \mathrm{d} z
$$




$$
\begin{aligned}
& f_{y z}=-\int_{z^{0}}^{z}\left(\frac{\partial}{\partial x}\left(\omega_{z} \sigma_{x x}+\tau_{x y}-\omega_{x} \tau_{x z}\right)+\frac{\partial}{\partial y}\left(\omega_{z} \tau_{x y}+\sigma_{y y}-\omega_{x} \tau_{y z}\right)+F_{Y}^{*}\right) \mathrm{d} z \\
& f_{z z}=-\int_{z^{0}}^{z}\left(\frac{\partial}{\partial x}\left(-\omega_{y} \sigma_{x x}+\omega_{x} \tau_{x y}+\tau_{x z}\right)+\frac{\partial}{\partial y}\left(-\omega_{y} \tau_{x y}+\omega_{x} \sigma_{y y}+\tau_{y z}\right)+F_{Z}^{*}\right) \mathrm{d} z
\end{aligned}
$$

Expanding the differentiation of Eq. (3.5) within the integrals gives

$$
\begin{aligned}
f_{x z}=-\int_{z^{0}}^{z}( & \sigma_{x x, x}-\omega_{z, x} \tau_{x y}-\omega_{z} \tau_{x y, x}+\omega_{y, x} \tau_{x z}+\omega_{y} \tau_{x z, x} \\
& \left.+\tau_{x y, y}-\omega_{z, y} \sigma_{y y}-\omega_{z} \sigma_{y y, y}+\omega_{y, y} \tau_{y z}+\omega_{y} \tau_{y z, y}+F_{X}^{*}\right) \mathrm{d} z \\
f_{y z}=-\int_{z^{0}}^{z}( & \omega_{z, x} \sigma_{x x}+\omega_{z} \sigma_{x x, x}+\tau_{x y, x}-\omega_{x, x} \tau_{x z}-\omega_{x} \tau_{x z, x} \\
& \left.+\omega_{z, y} \tau_{x y}+\omega_{z} \tau_{x y, y}+\sigma_{y y, y}-\omega_{x, y} \tau_{y z}-\omega_{x} \tau_{y z, y}+F_{Y}^{*}\right) \mathrm{d} z \\
f_{z z}=-\int_{z^{0}}^{z}( & -\omega_{y, x} \sigma_{x x}-\omega_{y} \sigma_{x x, x}+\omega_{x, x} \tau_{x y}+\omega_{x} \tau_{x y, x}+\tau_{x z, x} \\
& \left.-\omega_{y, y} \tau_{x y}-\omega_{y} \tau_{x y, y}+\omega_{x, y} \sigma_{y y}+\omega_{x} \sigma_{y y, y}+\tau_{y z, y}+F_{Z}^{*}\right) \mathrm{d} z
\end{aligned}
$$

in which a comma denotes differentiation with respect to the coordinates that follow. It is worth repeating that the integration of Eq. (3.14) is performed only within the layer of interest. Each expansion of the integrands of Eq. (3.14) consists of eleven terms. These terms are tabulated in Table 3.1

For solutions in which the in-plane stresses are known in analytical form, evaluating each of the terms of Table 3.1, and thus Eq. (3.14), is straight-forward. For solutions in which these stresses are only known at discrete positions within the body, such as a finite element solution, the process of evaluating Eq. (3.14) deserves additional attention.

\subsection{Application to Discretized Solutions}

Consider a discretized solution such as that from a plate finite element solution. Suppose the elements are constant strain, four-noded, reduced integration elements. Each element 
Table 3.1: Listing of Terms in Integrands of Eq. (3.14)

\begin{tabular}{cccc}
\hline$\#$ & $f_{x z}$ & $f_{y z}$ & $f_{z z}$ \\
\hline 1 & $\sigma_{x x, x}$ & $\omega_{z, x} \sigma_{x x}$ & $-\omega_{y, x} \sigma_{x x}$ \\
2 & $-\omega_{z, x} \tau_{x y}$ & $\omega_{z} \sigma_{x x, x}$ & $-\omega_{y} \sigma_{x x, x}$ \\
3 & $-\omega_{z} \tau_{x y, x}$ & $\tau_{x y, x}$ & $\omega_{x, x} \tau_{x y}$ \\
4 & $\omega_{y, x} \tau_{x z}$ & $-\omega_{x, x} \tau_{x z}$ & $\omega_{x} \tau_{x y, x}$ \\
5 & $\omega_{y} \tau_{x z, x}$ & $-\omega_{x} \tau_{x z, x}$ & $\tau_{x z, x}$ \\
\hline 6 & $\tau_{x y, y}$ & $\omega_{z, y} \tau_{x y}$ & $-\omega_{y, y} \tau_{x y}$ \\
7 & $-\omega_{z, y} \sigma_{y y}$ & $\omega_{z} \tau_{x y, y}$ & $-\omega_{y} \tau_{x y, y}$ \\
8 & $-\omega_{z} \sigma_{y y, y}$ & $\sigma_{y y, y}$ & $\omega_{x, y} \sigma_{y y}$ \\
9 & $\omega_{y, y} \tau_{y z}$ & $-\omega_{x, y} \tau_{y z}$ & $\omega_{x} \sigma_{y y, y}$ \\
10 & $\omega_{y} \tau_{y z, y}$ & $-\omega_{x} \tau_{y z, y}$ & $\tau_{y z, y}$ \\
\hline 11 & $-\rho a_{X}$ & $-\rho a_{Y}$ & $-\rho a_{Z}$ \\
\hline
\end{tabular}

contains one integration point for each sub-region through the thickness, say a region in the range $z^{*} \leq z \leq z^{* *}$, each integration point located at the element centroid. A sub-region could be an entire single layer of material, e.g. $z_{N} \leq z \leq z_{N-1}$ in Figure 3.2, in which case the layer would have one integration point through the thickness. Or, the sub-region could be a region within a layer, in which case the layer would have more than one integration point through the thickness. The former layer will be referred to as a physical layer, while the latter will be referred to as a numerical layer.

A cross-section of such a scheme is presented in Figure 3.3, where the finite elements lie on a reference surface $z=0$, and the thickness of the element is depicted as eight discrete layers, each with a single integration point. Although these layers are shown with equal

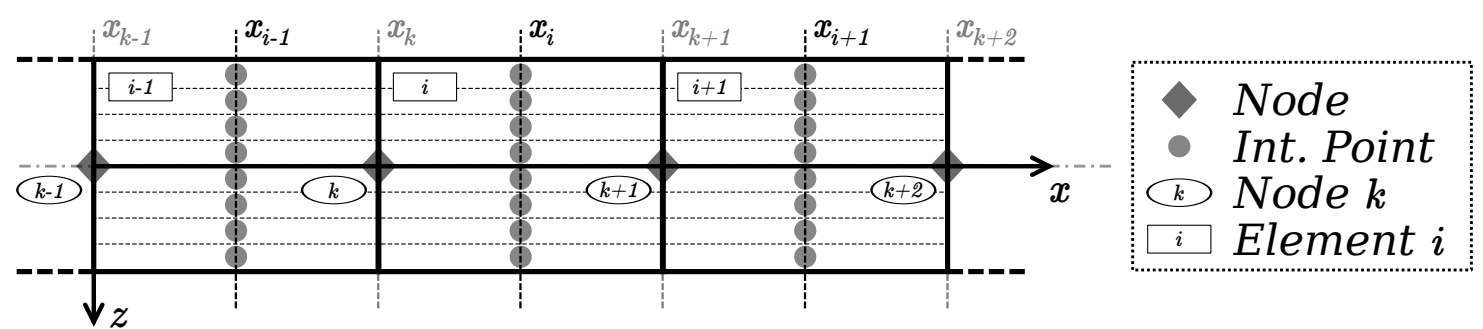

Figure 3.3: Example locations of known values from finite element solution, shown as a cross-section view of three shell elements. 
thicknesses, this need not be the case in general. As mentioned, a physical layer may be modeled with more than one numerical layer. Regardless, for a given finite element, the numerical values of a particular response within a numerical layer is constant within the plane and thickness of that layer, namely the value computed at the integration point. Finally, with a displacement-based solution with a finite element formulation based on the classical or first-order shear deformable plate theory, the displacements and rotations are given only at the element nodes, meaning they are constant through the thickness of the plate.

\subsubsection{Calculation of Terms of the Integrands}

When intralaminar stresses found by a numerical solution such as a finite element model are to be used in the stress recovery procedure, partial derivatives of these stresses, and other quantities of interest, are typically unavailable. Rather, values of stresses, displacements, and rotations are known only at discrete locations. Therefore, partial derivatives must be approximated by use of a finite-difference method.

Consider a finite element solution for which plate response is given at the nodal and integration point locations depicted in Figure 3.3. The $x-z$ plane of an eight-layer laminated plate is illustrated, along with discretization by way of three finite elements, $i-1$, $i$, and $i+1$, outlined with bold lines. The finite element nodes, identified by solid diamonds $k-1, k$, $k+1$, and $k+2$, are on the plate reference plane, $z=0$, while three sets of eight integration points, one integration point per layer and identified by solid circles, are distributed through the thickness at known $z$-locations. The $x$-locations of the three sets of integration points, which are element centroidal locations, are identified by the element numbers, namely, $x_{i-1}$, $x_{i}$, and $x_{i+1}$. Stresses are given at the integration points, while displacements and rotations are given at the nodal locations.

With each integral of Eq. (3.14), stresses, rotations, gradients of each, and accelerations must be determined. Values of stresses may be taken directly from the integration point 
data of the element of interest. Values of rotations and accelerations, which are located at the nodes of the element, are averaged from each node. In the case of the discretization of Figure 3.3, $\omega_{x}$ is found by

$$
\omega_{x}=\frac{\omega_{x}^{(k)}+\omega_{x}^{(k+1)}}{2}
$$

The gradients of rotations are found using the central difference method, such that $\omega_{y, x}$ is given by

$$
\frac{\partial \omega_{y}}{\partial x}=\frac{\omega_{y}^{(k+1)}-\omega_{y}^{(k)}}{x_{k+1}-x_{k}}
$$

Values of gradients of stresses are found by the central difference method, using elements on either side of the element of interest. For example, $\sigma_{x x, x}$ is found by

$$
\frac{\partial \sigma_{x x}}{\partial x}=\frac{\sigma_{x x}^{(i+1)}-\sigma_{x x}^{(i-1)}}{x_{i+1}-x_{i-1}}
$$

\subsubsection{Calculation of Rotations}

If a discretized solution provides all three rotations $\left(\omega_{x}, \omega_{y}\right.$, and $\omega_{z}$, for moderate rotations) at nodal locations as described by Figure 3.3, values of rotations may be used directly from the solution, and the calculation of gradients of rotations proceeds as discussed in Section 3.4.1. However, if rotations are not provided, the definitions of $\omega_{x}, \omega_{y}$, and $\omega_{z}$ in Eq. (3.2) may be used, with partial derivatives found by the finite difference method outlined in Section 3.4.1.

\subsubsection{Calculations Using Piecewise Constant Stresses}

Suppose data from a plate analysis are given for a laminate made of three physical layers, each of a different material. The three physical layers are modeled with four, one, and four numerical layers, respectively, so that there are nine integration points through the laminate. 
Stress data are given at each of those integration points. As shown in Figure 3.4, where the three layers are illustrated, a conservative assumption is that the stress values are constant within each layer, following the data computed at each of the nine integration points. For a classical plate analysis the shear and normal stresses are zero, and for a first-order shear deformation analysis the shear stresses are constant within a physical layer and the normal stress is zero. Either way, then, considering the integration of the first equilibrium equation (Eq. (3.1a)) given by $f_{x z}$ of Eq. (3.5a), all of the terms of the integrand are independent of

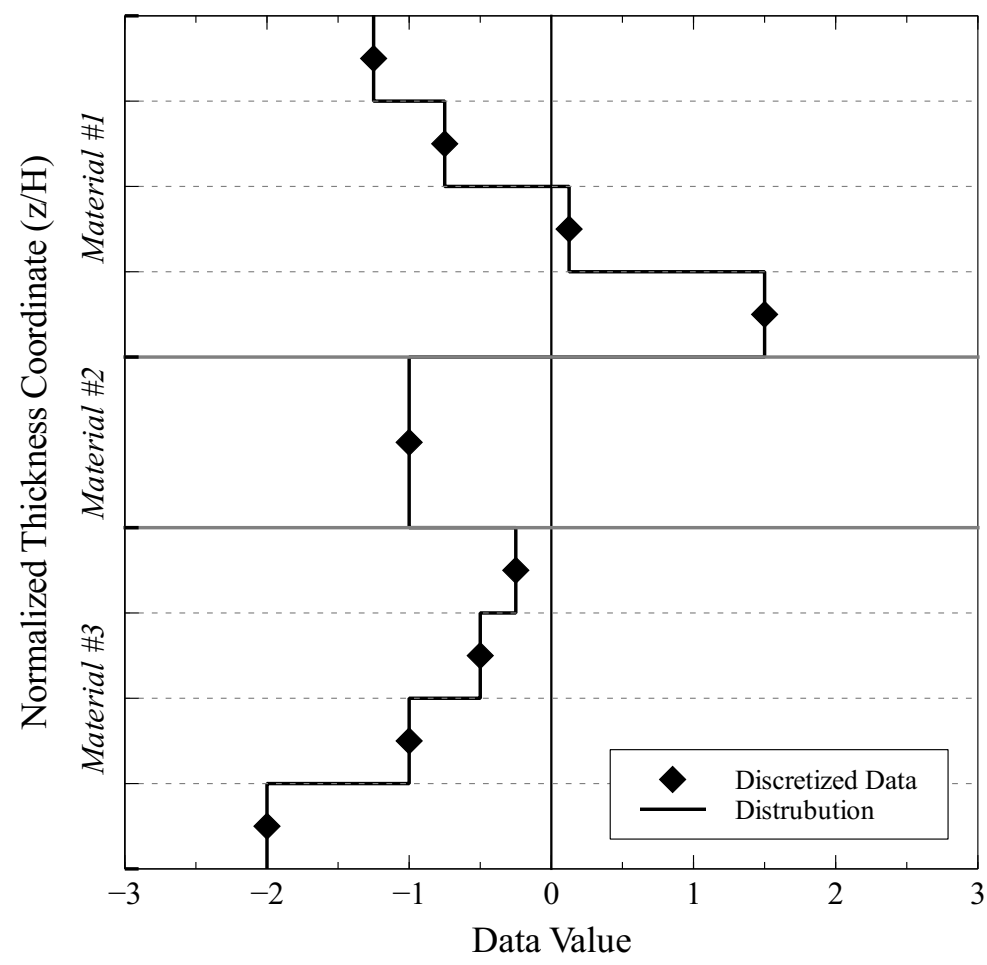

Figure 3.4: Example of Constant Data Distribution through Layers 
$z$ within each layer, and the integration can progress as follows:

$$
\begin{aligned}
f_{x z} & =-\int_{z^{0}}^{z}\left(\frac{\partial}{\partial x}\left(\sigma_{x x}-\omega_{z} \tau_{x y}+\omega_{y} \tau_{x z}\right)+\frac{\partial}{\partial y}\left(\tau_{x y}-\omega_{z} \sigma_{y y}+\omega_{y} \tau_{y z}\right)+F_{X}^{*}\right) \mathrm{d} z \\
& =-\left(\frac{\partial}{\partial x}\left(\sigma_{x x}-\omega_{z} \tau_{x y}+\omega_{y} \tau_{x z}\right)+\frac{\partial}{\partial y}\left(\tau_{x y}-\omega_{z} \sigma_{y y}+\omega_{y} \tau_{y z}\right)+F_{X}^{*}\right) \int_{z^{0}}^{z} \mathrm{~d} z \\
& =-\left(\frac{\partial}{\partial x}\left(\sigma_{x x}-\omega_{z} \tau_{x y}+\omega_{y} \tau_{x z}\right)+\frac{\partial}{\partial y}\left(\tau_{x y}-\omega_{z} \sigma_{y y}+\omega_{y} \tau_{y z}\right)+F_{X}^{*}\right)\left(z-z_{0}\right) \\
& =-C_{x z}\left(z-z_{0}\right)
\end{aligned}
$$

in which $C_{x z}$ is simply the sum of terms in the $f_{x z}$ column of Table 3.1:

$$
\begin{aligned}
C_{x z}=( & \sigma_{x x, x}-\omega_{z, x} \tau_{x y}-\omega_{z} \tau_{x y, x}+\omega_{y, x} \tau_{x z}+\omega_{y} \tau_{x z, x} \\
& \left.+\tau_{x y, y}-\omega_{z, y} \sigma_{y y}-\omega_{z} \sigma_{y y, y}+\omega_{y, y} \tau_{y z}+\omega_{y} \tau_{y z, y}+F_{X}^{*}\right)
\end{aligned}
$$

Analogous values of $C_{y z}$ and $C_{z z}$ are found in the same manner so that the recovered transverse stresses of Eq. (3.11) may be rewritten as

$$
\left\{\begin{array}{c}
\tau_{x z} \\
\tau_{y z} \\
\sigma_{z z}
\end{array}\right\}=\left(\begin{array}{ccc}
1 & \omega_{z} & -\omega_{y} \\
-\omega_{z} & 1 & \omega_{x} \\
\omega_{y} & -\omega_{x} & 1
\end{array}\right)\left\{\begin{array}{l}
-C_{x z}\left(z-z_{0}\right) \\
-C_{y z}\left(z-z_{0}\right) \\
-C_{z z}\left(z-z_{0}\right)
\end{array}\right\}+\left\{\begin{array}{c}
\tau_{x z}^{0} \\
\tau_{y z}^{0} \\
\sigma_{z z}^{0}
\end{array}\right\}
$$

\subsubsection{Calculations Using Piecewise Linear ${ }^{2}$ Stresses}

Consider the same three-layer, three-material laminate discussed in Section 3.4.3 regarding a constant distribution of data through each layer. Instead of the assumption of a constant distribution through each layer, suppose that a piecewise linear distribution of the stress data through the thickness of the laminate is assumed. This assumed distribution is valid if

\footnotetext{
${ }^{2}$ The term linear here is used to describe a variation that may be represented by an equation in the form of $y=m \cdot x+b$. This is not to imply that such a variation has the property of linearity such that $f(\alpha \cdot x)=\alpha \cdot f(x)$.
} 
the following criteria are met for each iteration:

- A linear distribution through the layer is interpolated between consecutive integration points if the material and fiber orientation at each integration point are identical.

- A linear distribution is extrapolated to interfaces between integration points whose material or fiber orientation differ.

- A constant distribution is assumed if an integration point does not neighbor another integration point with the same material and fiber orientation.

An example of such a distribution is shown in Figure 3.5, with identical discretized data to that of Figure 3.4. In such a distribution, stresses and stress gradients follow a linear distribution in each half of each numerical layer. For each half of the numerical layers, the

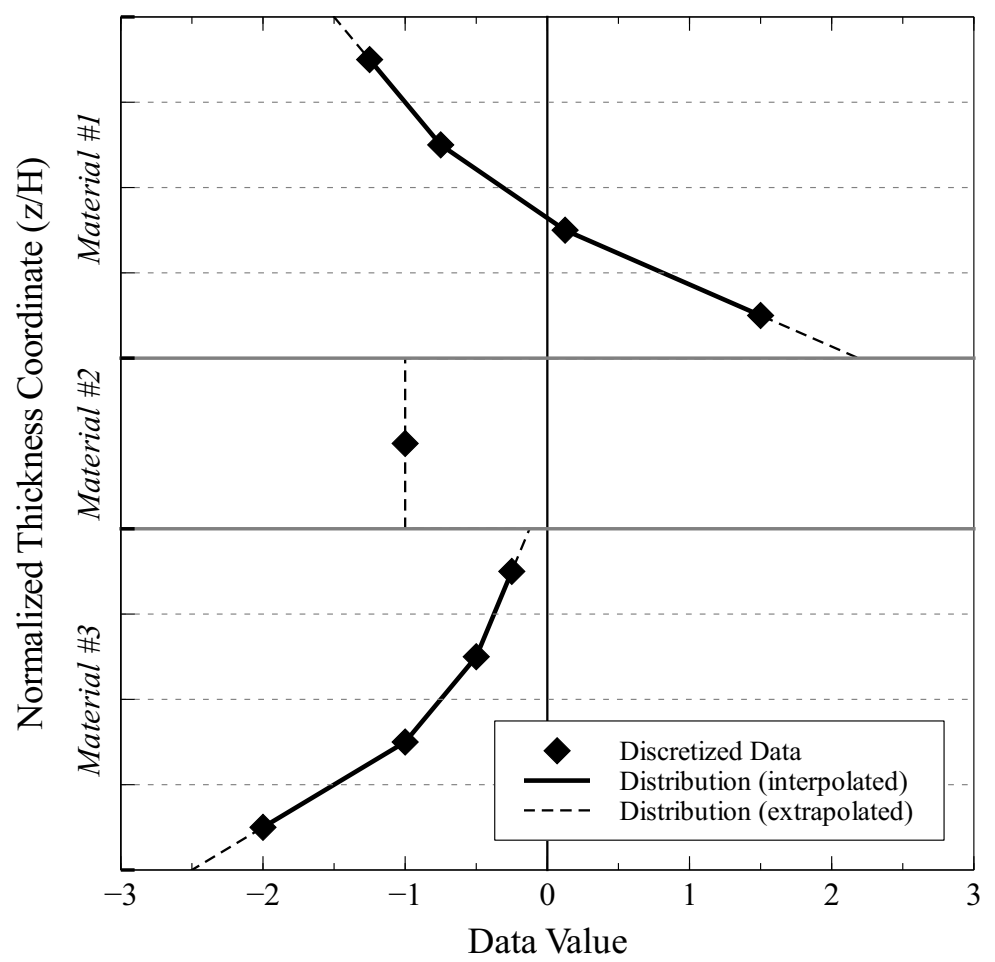

Figure 3.5: Example of Linear Data Distribution through Layers 
distribution of, for example, $\sigma_{x x}$ may then be given by

$$
\sigma_{x x}=m_{\sigma_{x x}} z+b_{\sigma_{x x}}
$$

where $m_{\sigma_{x x}}$ is the slope and $b_{\sigma_{x x}}$ is the intercept of a line describing the distribution of $\sigma_{x x}$ within the half numerical-layer. Let $M_{x z}$ be the sum of the slopes of the distributions of each term in the $f_{x z}$ column of Table 3.1. Likewise, let $B_{x z}$ be the sum of the intercepts of the distributions of each term in the $f_{x z}$ column of Table 3.1. With that notation, the integration of $f_{x z}$ of Eq. (3.5a) proceeds as

$$
\begin{aligned}
f_{x z} & =-\int_{z^{0}}^{z}\left(M_{x z} z+B_{x z}\right) \mathrm{d} z \\
& =-\int_{z^{0}}^{z}\left(M_{x z} z\right) \mathrm{d} z-\int_{z^{0}}^{z}\left(B_{x z}\right) \mathrm{d} z \\
& =-M_{x z} \int_{z^{0}}^{z} z \mathrm{~d} z-B_{x z} \int_{z^{0}}^{z} \mathrm{~d} z \\
& =-M_{x z}\left(\frac{z^{2}-z_{0}^{2}}{2}\right)-B_{x z}\left(z-z_{0}\right)
\end{aligned}
$$

Similar notation may be made for the remaining equilibrium equations so that the recovered transverse stresses in each half numerical-layer in the assumed piecewise linear distribution are given by

$$
\left\{\begin{array}{c}
\tau_{x z} \\
\tau_{y z} \\
\sigma_{z z}
\end{array}\right\}=\left(\begin{array}{ccc}
1 & \omega_{z} & -\omega_{y} \\
-\omega_{z} & 1 & \omega_{x} \\
\omega_{y} & -\omega_{x} & 1
\end{array}\right)\left\{\begin{array}{l}
-M_{x z}\left(\frac{z^{2}-z_{0}^{2}}{2}\right)-B_{x z}\left(z-z_{0}\right) \\
-M_{y z}\left(\frac{z^{2}-z_{0}^{2}}{2}\right)-B_{y z}\left(z-z_{0}\right) \\
-M_{z z}\left(\frac{z^{2}-z_{0}^{2}}{2}\right)-B_{z z}\left(z-z_{0}\right)
\end{array}\right\}+\left\{\begin{array}{c}
\tau_{x z}^{0} \\
\tau_{y z}^{0} \\
\sigma_{z z}^{0}
\end{array}\right\}
$$

It should be noted that when using stresses from a classical plate analysis in the recovery procedure, the transverse stresses are zero. Thus, in the first iteration, the constants $m$ and $b$ for the interlaminar stresses are zero. When using a plate analysis based on first-order shear deformation theory, the constant $m$ for the transverse shear stresses is zero. In subsequent iterations, however, the coefficients $m$ and $b$ for the interlaminar stresses are not generally 
zero.

\subsection{Contribution of Individual Terms}

Recall the integral functions of Eq. (3.14), and the terms which make up the integrands, listed in Table 3.1. As each integrand is simply a sum of the terms listed in Table 3.1, each term may be integrated individually through the thickness of the given layer. Thus, the contribution of each of those terms to the resulting recovered transverse stress may be determined. If this type of calculation is made for each term of the integrand, the total distribution may be found by simply summing the resulting distribution of each term. This, in fact, describes the implementation of the stress recovery procedure which is used for the results conveyed in the subsequent chapters. However, the explicit contribution of each term of the coefficients is discussed in-depth in Chapter 5 .

\subsection{Measurement of Procedure Accuracy}

For each recovered transverse stress based on the procedure developed here, the corresponding traction at the lower plate surface must be known (see Figure 3.2) ${ }^{3}$. However, the tractions at the upper surface are not included in the formulation; thus, the values of the recovered stresses at the upper surface compared to their actual known values may be used as an indication of the accuracy of the recovery process. This will be done in subsequent discussions.

\footnotetext{
${ }^{3}$ Again, the recovery procedure presented here could just as easily have used the upper plate surface as the reference surface. In that case, the tractions at the upper surface would be used in the recovery of the transverse stresses, while the tractions at the lower surface may be used to compare to the recovered stresses at that surface.
} 


\section{Chapter 4}

\section{Stress Recovery in a}

\section{Semi-Infinite Domain}

The results of this section have been published [52] as:

T. B. Hartman, M. W. Hyer, and S. W. Case (May 2012a). Geometrically Nonlinear Stress Recovery in Composite Laminates. AIAA Journal (50)5: 1156-1168 and presented [53] as:

T. B. Hartman, M. W. Hyer, and S. W. Case (Apr. 2011). Stress Recovery in Composite Laminates. In: 52nd AIAA/ASME/ASCE/AHS/ASC Structures, Structural Dynamics, and Materials Conference. Denver, CO

As a first step to applying the geometrically nonlinear stress recovery procedure, an example problem of a semi-infinite plate is examined. This allows simplification of the stress recovery procedure by reducing the algebraic overhead required, while retaining the necessary components to examine the applicability of the geometrically nonlinear stress recovery procedure. The procedure will be applied to each a plate-type analytical solution as well as a plate-type finite element solution. The former provides functions of the stresses which may be differentiated analytically, while the latter provides plate response only at 
discrete locations such that the finite difference scheme of Section 3.4 must be employed to calculate the necessary derivatives. Recovered stresses from both solutions are compared to results from a benchmark finite element solution.

\subsection{Description of Example Problems}

The example problems to which the stress recovery procedure will be applied are based on the plate geometry shown in Figure 4.1. The plate considered is symmetrically-laminated, infinite in extent in the $y$-direction, of span $a$ in the $x$-direction, of thickness $H$, and centrally loaded over part of its span. The $x y z$ coordinate system is located at the plate's mid-surface, the reference surface of the analysis. The displacements in the $x$-, $y$-, and $z$-directions are denoted as $u, v$, and $w$. As shown in Figure 4.2, the plate is partially loaded with either a uniformly-distributed transverse load $q_{0}$, which remains oriented in the $z$-direction, or a uniformly-distributed shear load $t_{0}$, which follows the deformations of the plate, that is, a follower load. In either case, the loads are applied in a quasi-static manner so that inertial effects may be ignored. These two loading conditions offer a variety of plate responses; in particular, interlaminar stress states. The span of the partial load is $\alpha \cdot a, 0<\alpha \leq 1$. The plate is pinned at $x= \pm a / 2$ so motion in the $x$ - and $z$-direction is constrained. These restraints lead to nonlinear geometric effects in the form of membrane forces within the plate when the plate deflects out of plane. Here the out-of-plane deflections will be limited to the

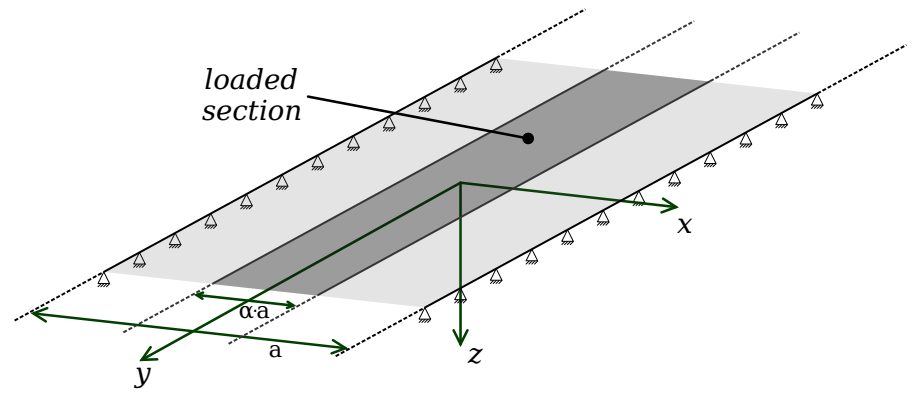

Figure 4.1: View of semi-infinite plate. 
extent that the rotations associated with the plate deformations can be considered moderate.

The plate is assumed to behave elastically, and because of the plate's infinite extent in the $y$-direction, it will be assumed that

$$
\frac{\partial(\quad)}{\partial y}=0
$$

where ( ) represents any component of the plate's response. To further simplify the problem, it will be assumed that the displacement in the $y$-direction is everywhere restrained, that is,

$$
v(x, y, z)=0
$$

Because of the orientation of the $x y z$ analysis coordinate system, the in-plane stress components $\sigma_{x x}, \sigma_{y y}$, and $\tau_{x y}$ will be referred to as the intralaminar stresses, while the out-of-plane stress components $\tau_{x z}, \tau_{y z}$, and $\sigma_{z z}$ will be referred to as the interlaminar stresses, where standard notation for the stress components is used. Of the three interlaminar stress components, the discussion here will focus on the two primary components for the example

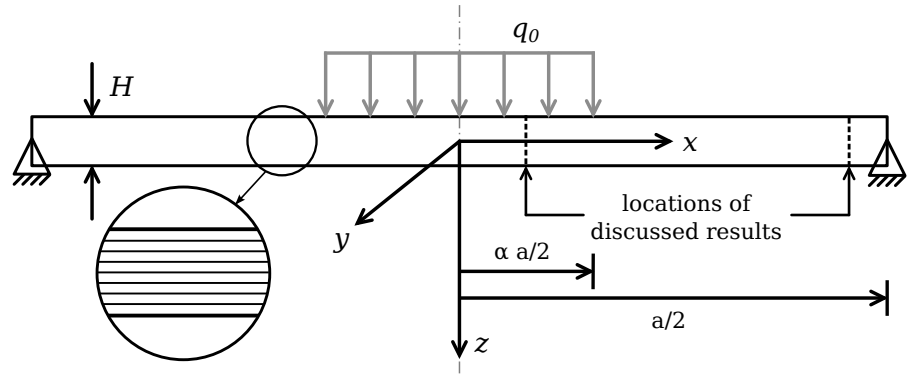

(a) Plate with transverse load.

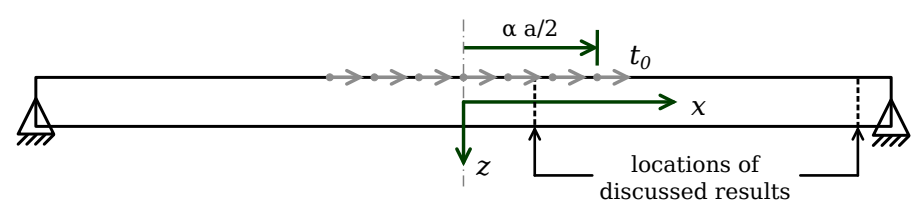

(b) Plate with shear load.

Figure 4.2: Cross section of semi-infinite plate. 
problems, namely, the shear stress $\tau_{x z}$ and the normal stress $\sigma_{z z}$. Results for these stresses will discussed for the span-wise locations shown in Figure 4.2, specifically, within the loaded portion of the plate at $x / a=0.05$, and near the supports at $x / a=0.45$. These locations are selected because they offer a variety of boundary conditions for the interlaminar stresses on the top and bottom plate surfaces and a variety of interlaminar stress responses. Overall, these problems, though simplified, have the features necessary to verify that the iterative stress recovery procedure presented is accurate and the extension to more complex problems is simply a matter of keeping track of more algebra.

\subsection{Simplification of Stress Recovery Procedure}

Because of the infinite extent of the plate in the $y$-direction, the rotations (c.f. Eq. (3.2)) about the $x$ - and $z$-axes are zero,

$$
\omega_{x}=\omega_{z}=0
$$

as are any gradients in $Y$. With these simplifications, the integrated geometrically nonlinear equilibrium equations, Eq. (3.4), for $\tau_{x z}$ and $\sigma_{z z}$ for the quasi-static semi-infinite example problems become

$$
\begin{aligned}
\left.\left(\tau_{x z}+\omega_{y} \sigma_{z z}\right)\right|_{z^{0}} ^{z} & =-\int_{z^{0}}^{z}\left(\frac{\partial}{\partial x}\left(\sigma_{x x}+\omega_{y} \tau_{x z}\right)\right) \mathrm{d} z \\
\left.\left(-\omega_{y} \tau_{x z}+\sigma_{z z}\right)\right|_{z^{0}} ^{z} & =-\int_{z^{0}}^{z}\left(\frac{\partial}{\partial x}\left(-\omega_{y} \sigma_{x x}+\tau_{x z}\right)\right) \mathrm{d} z
\end{aligned}
$$

Applying the recovery procedure described in Section 3.2 results in the system of equations

$$
\left\{\begin{array}{c}
\tau_{x z}^{(i)} \\
\sigma_{z z}^{(i)}
\end{array}\right\}=\left(\begin{array}{cc}
1 & -\omega_{y} \\
\omega_{y} & 1
\end{array}\right)\left\{\begin{array}{c}
f_{x z}^{(i-1)} \\
f_{z z}^{(i-1)}
\end{array}\right\}+\left\{\begin{array}{c}
\tau_{x z}^{0} \\
\sigma_{z z}^{0}
\end{array}\right\}
$$


with the terms of the integrands $f_{x z}$ and $f_{z z}$ listed in Table 4.1. The stress recovery procedure for the semi-infinite example problems is then identical to that described by Section 3.2, albeit with reduced algebraic overhead.

\subsection{Results}

\subsubsection{Problem Parameters}

Results are obtained for three different plates: an isotropic plate made of a single layer of steel and two laminated composite plates made of eight layers of graphite-epoxy, one with a stacking sequence of $\left[0^{\circ} / 90^{\circ}\right]_{2 S}$, the other with a stacking sequence of $\left[ \pm 30^{\circ} / 0^{\circ} / 90^{\circ}\right]_{S}$. These plates will be referred to as isotropic, cross-ply, and angle-ply plates, respectively. All plates are of width $a=1.0 \mathrm{~m}$ and the partial uniform load covers $20 \%$ of the plate width, that is, $\alpha$ of Figures 4.1 and 4.2 is 0.2 . The total thickness of the steel plate is $0.010 \mathrm{~m}$. The graphiteepoxy layers are $150 \mu \mathrm{m}$ thick, so that the thickness of each laminated composite plate is $0.0012 \mathrm{~m}$. The material properties are given in Table 4.2, while the remaining parameters are listed in Table 4.3. For the composite plate the fiber orientation in the layers is measured relative to the $+x$-direction, where a positive fiber orientation corresponds to a positive rotation about the $+z$-axis, the usual convention.

Table 4.1: Listing of Terms in Integrands of Eq. (4.4)

\begin{tabular}{clc}
\hline$\#$ & \multicolumn{1}{c}{$f_{x z}$} & $f_{z z}$ \\
\hline 1 & $\sigma_{x x, x}$ & $-\omega_{y, x} \sigma_{x x}$ \\
2 & & $-\omega_{y} \sigma_{x x, x}$ \\
3 & $\omega_{y, x} \tau_{x z}$ & \\
4 & $\omega_{y} \tau_{x z, x}$ & $\tau_{x z, x}$ \\
\hline
\end{tabular}


Table 4.2: Material properties.

\begin{tabular}{lcrl}
\hline Material Name & Elastic Property & Value & Unit \\
\hline \multirow{2}{*}{ Steel } & $E$ & 210.0 & $\mathrm{GPa}$ \\
& $\nu$ & 0.3 & \\
\hline \multirow{6}{*}{ Graphite-epoxy } & $E_{1}$ & 155.0 & $\mathrm{GPa}$ \\
& $E_{2}$ & 12.1 & $\mathrm{GPa}$ \\
& $E_{3}$ & 12.1 & $\mathrm{GPa}$ \\
& $\nu_{23}$ & 0.458 & \\
& $\nu_{13}$ & 0.248 & \\
& $\nu_{12}$ & 0.248 & \\
& $G_{23}$ & 3.20 & $\mathrm{GPa}$ \\
& $G_{13}$ & 4.40 & $\mathrm{GPa}$ \\
& $G_{12}$ & 4.40 & $\mathrm{GPa}$ \\
\hline
\end{tabular}

Table 4.3: Plate parameters.

\begin{tabular}{lccccc}
\hline Plate Name & $a$ & $\alpha$ & $H$ & $q_{0}$ & $t_{0}$ \\
\hline Isotropic & $1.0 \mathrm{~m}$ & 0.2 & $0.0100 \mathrm{~m}$ & $250 \mathrm{kPa}$ & $2.5 \mathrm{MPa}$ \\
Cross-ply & $1.0 \mathrm{~m}$ & 0.2 & $0.0012 \mathrm{~m}$ & $2.5 \mathrm{~Pa}$ & $2.5 \mathrm{kPa}$ \\
Angle-ply & $1.0 \mathrm{~m}$ & 0.2 & $0.0012 \mathrm{~m}$ & $2.5 \mathrm{~Pa}$ & $2.5 \mathrm{kPa}$ \\
\hline
\end{tabular}

\subsubsection{Details of Plate Finite Element Model}

To investigate the developed stress recovery procedure when using intralaminar stresses computed by a numerical approach, the example problems are modeled as plates using the commercial code LS-DYNA [54]. The model consists of 50 Belytschko-Tsay four-node shell elements spanning the width of the plate, with a single element in the infinite-length direction. To simulate the infinite extent of the plate in the $y$-direction, nodes with the same initial $x$-coordinate are constrained to have equal displacements in the $x$-direction, equal displacements in the $z$-direction, and equal rotations about the $y$-axis. Additionally, all nodes are constrained from motion in the $y$-direction, as well as from rotation about the $x$ and $z$ axes. These restraints satisfy the conditions required by Eqs. (4.1) and (4.2). To satisfy the pinned boundary conditions at the plate edges, nodes at $x= \pm a / 2$ are constrained 
from motion in the $x$ - and $z$-directions. Each element is $0.02 \mathrm{~m}$ along the $x$-direction, and $0.04 \mathrm{~m}$ along the $y$-direction.

The transverse loading is modeled with non-follower forces acting in the $+z$-direction on the appropriate nodes within the loaded portion of the plate. To aid in the application of the follower shear load within the loaded portion of the plate, four-node rigid shell elements are attached normal to the reference surface of the plate, extending in the $-z$-direction. These rigid elements remain normal to the reference surface of the plate. Point loads, initially in the $x$-direction, act on the rigid elements a distance of $H / 2$ from the plate reference to simulate the shear force acting on the top surface of the plate.

The isotropic plate is modeled with eight integration points through the thickness, all located at the centroid of each plate element. The composite plates are modeled by specifying the thickness and fiber orientation of each layer. There is one integration point at the center of each layer, for a total of eight integration points through the thickness, again at the centroid of each plate element. All calculations are made using the LS-DYNA implicit solver. Geometrically linear solutions, considered for comparison, are obtained by applying a load six orders of magnitude less than the desired load, then increasing the resulting displacements and stresses by six orders of magnitude. For the geometrically linear stress recovery procedure, the rotation $\omega_{y}$ is set to zero in Eq. (3.1) and subsequent related equations. Iteration is not necessary.

\subsubsection{Details of Benchmark Three-Dimensional Finite Element Model}

The benchmark three-dimensional finite element solution uses eight-node generalized plane strain elements from the Abaqus [55] library (Abaqus element ID: CPEG8) to model the $x-z$ plane of the plate (see Figure 4.2). The element uses nine integration points, including a single point at the centroid of the element, at which all six stress components are reported. The single reference node required to model generalized plane strain is constrained from all motion. Nodes at $x= \pm a / 2, z=0$ (see Figure 4.2) are constrained from motion in the 
$x$ - and $z$-directions. Loads are applied to nodes within the loaded portion of the plate at $z=-H / 2$ as either non-following nodal forces in the $+z$-direction or follower nodal forces in the $+x$-direction. The isotropic plate is modeled with 3,010 elements of equal dimension in the span-wise direction of the plate and with sixteen elements of equal dimension in the thickness direction, resulting in approximately square elements. The composite plates are modeled using orthotropic engineering properties for the $0^{\circ}$ and $90^{\circ}$ layers and anisotropic stiffness components for the $\pm 30^{\circ}$ layers, all specified in the $x y z$ coordinate frame. The composite plates have 30,010 elements of equal dimension in the span-wise direction of the plate and sixteen elements of equal dimension in the thickness direction (two elements per composite layer). The composite plate are composed of more elements along the width of the plate to keep the element aspect ratio approximately equal to that of the mesh for the isotropic plate. All calculations are made using the implicit solver. The geometrically linear benchmark solutions are obtained by not invoking the geometrically nonlinear option in Abaqus. The finite-element code Abaqus was used to obtain the benchmark results simply because of familiarity with the generalized plane deformation elements in that code and their representation of off-axis layers.

\subsubsection{Details of Closed-Form Plate Solutions}

The closed-form plate response due to the $z$-direction transverse loading is developed by integrating the governing differential equilibrium equations and applying the associated boundary conditions. The result is an exact solution, in closed form, for the plate stresses and deformations within the context of classical laminated plate theory. The closed-form solution for the shear loading is an approximate solution based on the Rayleigh-Ritz technique. Details of these two closed-form solutions are given in Appendix B. Interlaminar stress results based on using these closed-form solutions in the integrands of Eq. (4.4), and the ensuing iteration scheme, are illustrated in the figures to follow, along with the interlaminar stresses computed using plate finite element results in the developed procedure, and results from the 
benchmark finite element model.

\subsubsection{Stress Recovery Results}

The nomenclature shown in Table 4.4 will be used to refer to the results from each of the previously described models of the example problems. Results of the stress recovery procedure based on the intralaminar stresses computed with plate elements in the finite element code LS-DYNA will be referred to simply as the "numerical" results. Interlaminar stress results computed by the benchmark three-dimensional solution based on solid elements in the finite element code Abaqus will be referred to as the "benchmark" results. Finally, results of the stress recovery procedure based on the intralaminar stresses computed by the closed-form solutions will each be referred to as "closed-form" results.

As an introduction to the results, but to also illustrate the influence of geometrically nonlinear effects on the problems being discussed, the closed-form solutions for the out-ofplane plate deflections across the span of the isotropic plate are shown in Figure 4.3 for both the transverse and shear loading cases. In Figure 4.3 the horizontal axes are the spanwise positions across the plate normalized by the span dimension $a$, and the vertical axes are the out-of-plane deflections normalized by the plate thickness $H$. Note that positive deflections are plotted downward to match the analysis coordinate system of Figures 4.1 and 4.2. Dashed lines represent results from the geometrically linear solutions, while solid lines represent results from the geometrically nonlinear solutions. The load levels for the linear and nonlinear solutions are the same and are given in Table 4.3. The plots in Figure 4.3

Table 4.4: Solution nomenclature.

\begin{tabular}{lccccc}
\hline \multirow{2}{*}{ Solution Type } & \multirow{2}{*}{ Written Name } & \multicolumn{2}{c}{ Plot Name } & \multicolumn{2}{c}{ Plot Style } \\
& & Linear & Nonlinear & Linear & Nonlinear \\
\hline Finite elements, plate theory & numerical & Num. & Num. NL & $\boldsymbol{\square}$ & $\mathbf{\square}$ \\
Benchmark, 3D finite elements & benchmark & Bench. & Bench. NL & $\boldsymbol{\Delta}$ & $\boldsymbol{\Delta}$ \\
Exact and Rayleigh-Ritz & closed-form & CF & CF NL & $-\ldots$ & - \\
\hline
\end{tabular}


typify the results of the composite plates as well. It is worth noting the effect of the geometric nonlinearities. In the transverse loading case, Figure 4.3a, the deflections predicted by the nonlinear solution are substantially less than those of the linear solution. This result is due to the addition of membrane stresses, which provide geometric stiffening. The forms of the deflected shapes, however, are similar. In the shear loading case, Figure 4.3b, the deflections predicted by the nonlinear solution are greater than those of the linear solution. Additionally, the forms of the deflections are vastly different, as the follower loading rotates with the plate so that a component of the loading acts in the $z$-direction to contribute to the out-of-plane displacement of the plate. For the cases illustrated in Figure 4.3 the deflections for the nonlinear solutions are of the order of one plate thickness. The interlaminar stresses for these deformed configurations are discussed next. The deflections and rotations from Figure 4.3 are summarized in Table 4.5, as are results from subsequent figures.

\subsubsection{Transverse Loading Case $\left(q_{0} \neq 0, t_{0}=0\right)$}

Results of the various calculations for the interlaminar shear and normal stresses for the isotropic, cross-ply, and angle-ply plates for the transverse load case are illustrated in Figures $4.4 \mathrm{a}$ to $4.4 \mathrm{c}$, respectively. The through-thickness stress distributions are shown for the location under the load, at $x / a=0.05$ (see Figure 4.2a). This span-wise location corresponds

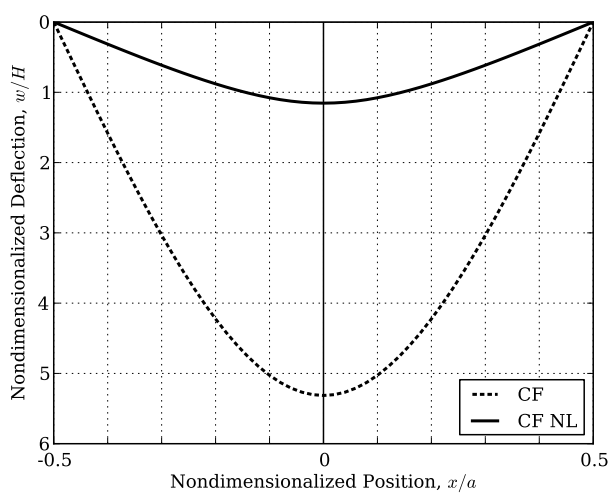

(a) Transverse loading.

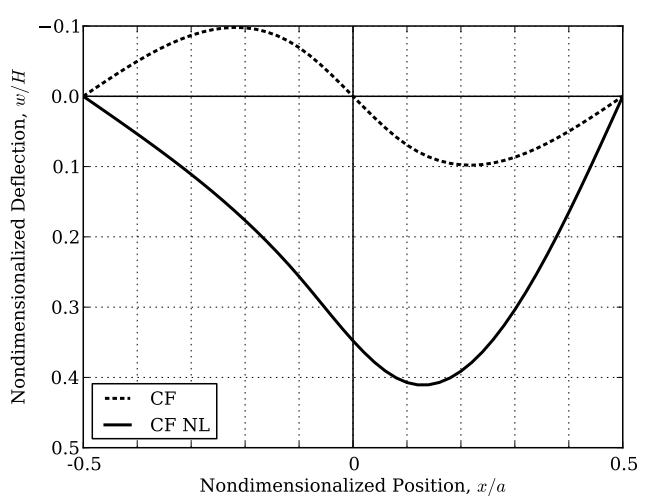

(b) Shear loading.

Figure 4.3: Deflection of isotropic plate. 
Table 4.5: Example problems solution kinematics.

\begin{tabular}{|c|c|c|c|c|c|}
\hline Loading & Nonlinearity & Plate & $\begin{array}{c}\text { Center Deflection } \\
w / H\end{array}$ & \multicolumn{2}{|c|}{$\omega_{y}$} \\
\hline \multirow{6}{*}{ normal } & & isotropic & 5.314 & & \\
\hline & linear & cross-ply & 0.533 & \multirow{2}{*}{\multicolumn{2}{|c|}{-}} \\
\hline & & angle-ply & 0.601 & & \\
\hline & \multirow{3}{*}{ nonlinear } & isotropic & 1.154 & $-7.818 \mathrm{E}-3$ & $-3.151 \mathrm{E}-2$ \\
\hline & & cross-ply & 0.397 & $-2.675 \mathrm{E}-4$ & $-1.407 \mathrm{E}-3$ \\
\hline & & angle-ply & 0.416 & $-2.824 \mathrm{E}-4$ & $-1.468 \mathrm{E}-3$ \\
\hline \multirow{6}{*}{ shear } & \multirow{3}{*}{ linear } & isotropic & 0 & & \\
\hline & & cross-ply & 0 & \multirow{2}{*}{\multicolumn{2}{|c|}{ - }} \\
\hline & & angle-ply & 0 & & \\
\hline & \multirow{3}{*}{ nonlinear } & isotropic & 0.348 & $6.160 \mathrm{E}-3$ & $-1.662 \mathrm{E}-2$ \\
\hline & & cross-ply & 0.469 & $8.959 \mathrm{E}-4$ & $-2.623 \mathrm{E}-3$ \\
\hline & & angle-ply & 0.501 & $9.879 \mathrm{E}-4$ & $-2.822 \mathrm{E}-3$ \\
\hline
\end{tabular}

to the location of an element centroid in both the plate and benchmark finite element models. The stresses have been normalized by the distributed load $q_{o}$, and the through-thickness locations have been normalized by the plate thickness, $H$. A positive through-thickness location is plotted downward to match the analysis coordinate system of Figures 4.1 and 4.2. In the figures, the solid and open square symbols represent results obtained by using the intralaminar stresses from the numerical plate model in the geometrically nonlinear and geometrically linear stress recovery procedures, respectively. The solid and open triangular symbols represent results obtained from the benchmark three-dimensional Abaqus finite element solution for geometrically nonlinear and geometrically linear analyses, respectively. The solid lines represent results obtained by using the intralaminar stresses from the closed-form plate solution in the stress recovery procedure, with consideration of geometric nonlinearities. The dashed lines represent results obtained when geometric nonlinearities are ignored.

There are several overall comments to be made regarding results shown in these figures. First, there is excellent agreement among the three stress calculations for any particular interlaminar stress component for any particular plate. The interlaminar shear and nor- 

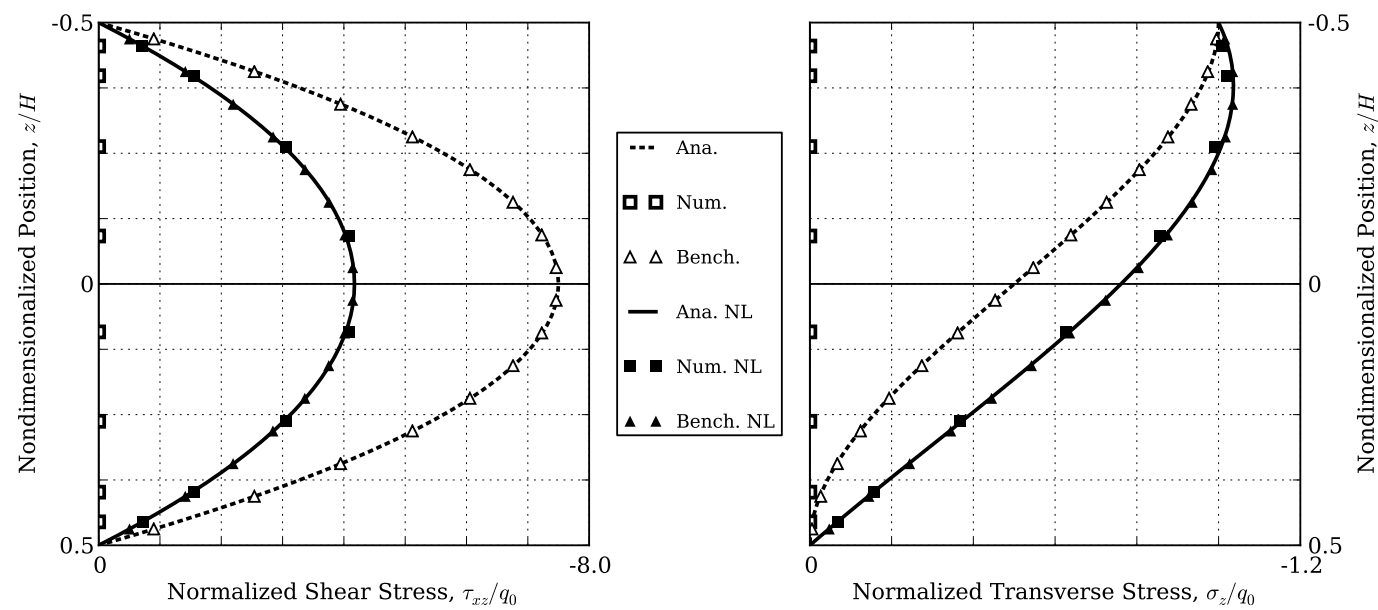

(a) Isotropic plate.
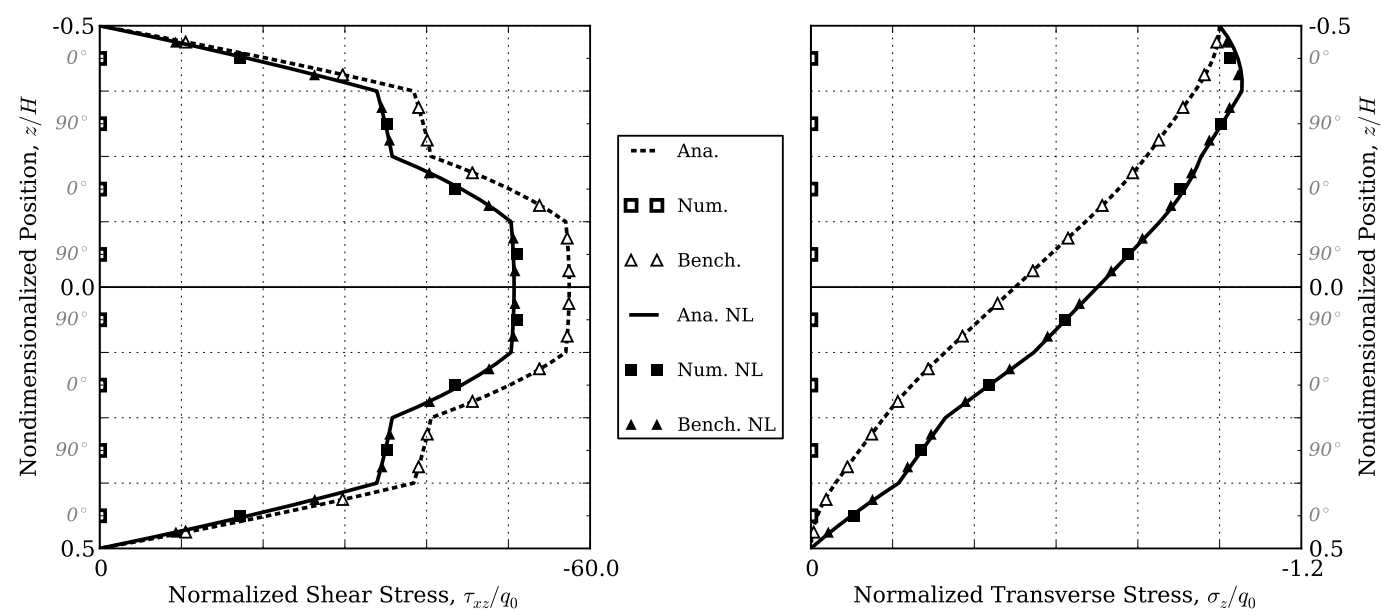

(b) Cross-ply plate.
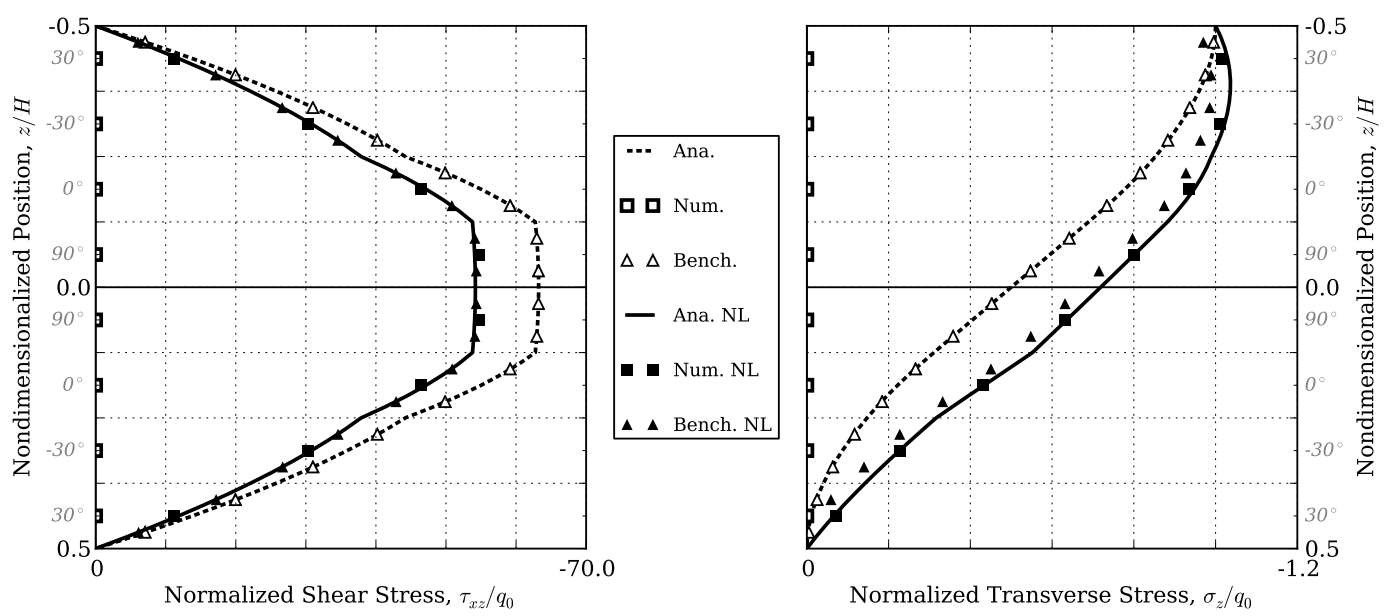

(c) Angle-ply plate.

Figure 4.4: Interlaminar stresses at $x=0.05 \cdot a$ due to transverse loading. 
mal stresses obtained by using the numerical plate model intralaminar stresses in the stress recovery procedure, as discussed in Section 3.4, agree well with results obtained from the benchmark three-dimensional finite element model, and by using the closed-form plate solution intralaminar stresses in the stress recovery procedure. It can thus be concluded that for the specific loading and boundary conditions considered here, the stress recovery procedure works well when intralaminar stresses from the numerical plate model are used, the focus of this paper. Second, there are noticeable differences between the results when including geometrically nonlinear effects and when they are not included. It can thus also be concluded that for the specific loading and boundary conditions, geometric nonlinearities should not be ignored. This reinforces the observations made in relation to Figure 4.3. Recall, the thickest plate considered (isotropic) is $1 \mathrm{~m}$ wide and $10 \mathrm{~mm}$ thick. The deflections of each of the plates, as mentioned, are on the order of one plate thickness, not large, yet geometric nonlinearities have an influence. Third, it is important to be aware of the difference in the normalized values of the stress components. In Figure 4.4, the normalized shear stresses $\tau_{x z}$ are larger than the normalized normal stresses $\sigma_{z z}$. Fourth, the boundary conditions at the top surfaces of the various plates as computed by the stress recovery procedure using either the numerical plate element stresses or the closed-form solution match well with the actual boundary conditions on those surfaces. Recall, with the developed stress recovery procedure the boundary conditions on the top surface do not enter into the calculations and thus are not explicitly enforced.

Considering the details for the isotropic plate in Figure $4.4 \mathrm{a}$, the zero shear stress boundary condition is enforced on the bottom surface as part of the stress recovery procedure, and the shear stress is correctly calculated to be zero on the top surface. The normal stress is smaller than the shear stress and is correctly predicted. The zero normal stress boundary condition is enforced on the bottom surface and the normal stress is correctly calculated to match a compressive value equal to the distributed transverse load on the top surface, that is, $\sigma_{z z} / q_{o}=-1$. It is interesting to note that when geometric nonlinearities are considered, 
the maximum compressive normal stress does not occur at the top surface, rather it occurs just below the top surface.

For the cross-ply plate, Figure 4.4b, the stress recovery procedure results in stresses that correlate very well with the benchmark solutions in regard to through-thickness rates of change. The normal stress is similar to the isotropic plate case, including the fact that the maximum stress occurs just below the top surface. For the cross-ply plate the normal stress is considerably smaller than the shear stress, more so than for the isotropic plate in Figure 4.4a due to the composite plate being thinner than the isotropic plate for the numerical examples considered here. For the geometrically nonlinear case and for the low stress levels near the bottom surface of the plate, there is a lack of smoothness in the through-thickness distribution due to the change of material properties from one layer to the next. The stress recovery procedure predicts the lack of smoothness quite well. Both computed shear and normal stresses match the boundary conditions on the top surface.

For the angle-ply plate (Figure 4.4c) the comments regarding normal stress for the crossply plate apply except for the fact that the results obtained from the benchmark threedimensional finite element model are shifted slightly to the left. As a result, the boundary conditions are not satisfied with the three-dimensional model.

In Figure 4.5, attention for the transverse loading case now turns to the through-thickness stress distributions near the plate support, $x / a=0.45$. This location also corresponds to the location of element centroids in the finite element models. At this location the shear stress is twice as large as at the under-load location considered in Figure 4.4. Furthermore, the boundary conditions at the top and bottom surface for both the shear and normal stresses are zero. Considering the normal stress component, since the boundary values of this stress are zero at both plate surfaces, the normal stress within the thickness is not expected to be large. As seen, this is indeed the case. For the geometrically linear cases the normal stresses are zero for all three plates, and for the geometrically nonlinear cases the magnitudes of the normal stresses are many times smaller than the magnitudes of the shear stresses. The 
two geometrically nonlinear finite element results reflect the $S$-shaped distribution for the isotropic plate, but neither satisfies the boundary conditions on both the top and bottom plate surfaces. For the cross-ply and angle-ply plates, where the normal stress component is even smaller in relation to the shear stress, the two geometrically nonlinear finite element calculations are not accurate. In fact, so as to not distort the scale of the normal stress plots for the cross- and angle-ply plates, the benchmark solution for the geometrically nonlinear case is not shown. For the cross-ply plate the normal stress distribution as computed by the benchmark solution for the geometrically nonlinear case is slightly $S$-shaped and centered around a normalized value of $\sigma_{z z} / q_{0}=0.4$. For the angle-ply plate the normal stress distribution is also slightly $S$-shaped and centered around a normalized value of $\sigma_{z z} / q_{0}=2.5$. Given the magnitude of the normal stress in relation to the shear stress, these errors are not considered a significant problem.

\subsubsection{Shear Loading Case $\left(q_{0}=0, t_{0} \neq 0\right)$}

The through-thickness shear and normal stress distributions for the isotropic, cross-ply, and angle-ply plates are illustrated in Figures 4.6a to 4.6c for the shear loading cases and for the location under the shear load. The format of the figures is the same as for the transverse loading except the stresses are normalized by the shear load $t_{o}$. It is important to note that the solid and dashed lines in these figures represent the results of using the Rayleigh-Ritz approximate solution to the plate problem. The Rayleigh-Ritz solution is still in closed form, but because it is approximate, some disagreement between the results based on this solution and the results from the benchmark solution might be expected, whereas this was not generally the case for the transverse loading results in Figures 4.4 and 4.5.

Considering the shear stress distributions in Figure 4.6, since the shear load is applied at the top surface of the plate, the normalized shear stress should be unity at that throughthickness location. An inspection of Figure 4.6 shows that this is indeed the case. Recall, as applied here, it is the zero shear stress boundary condition on the bottom surface that is 

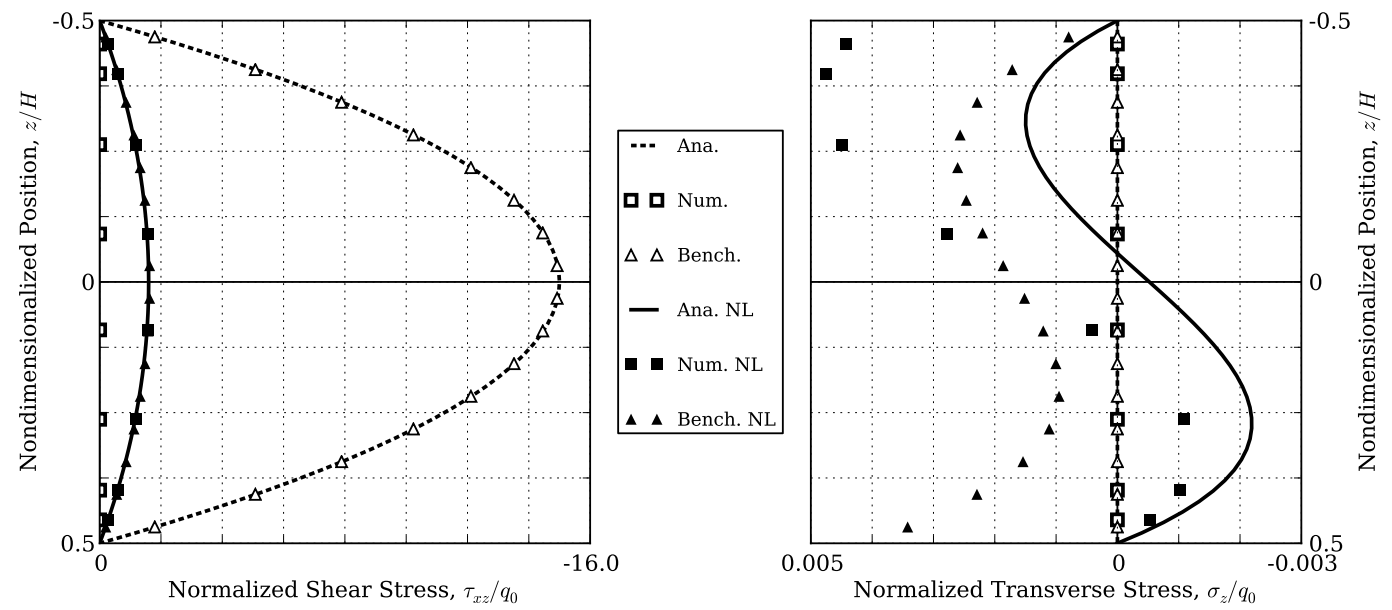

(a) Isotropic plate.
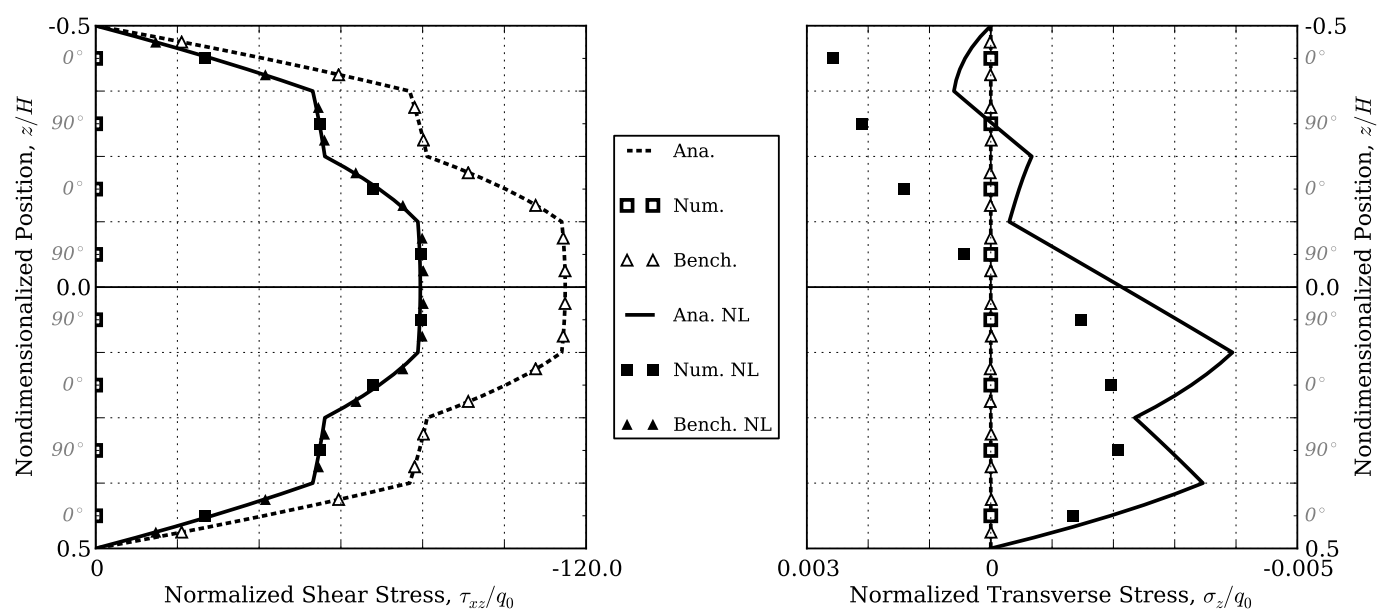

(b) Cross-ply plate.
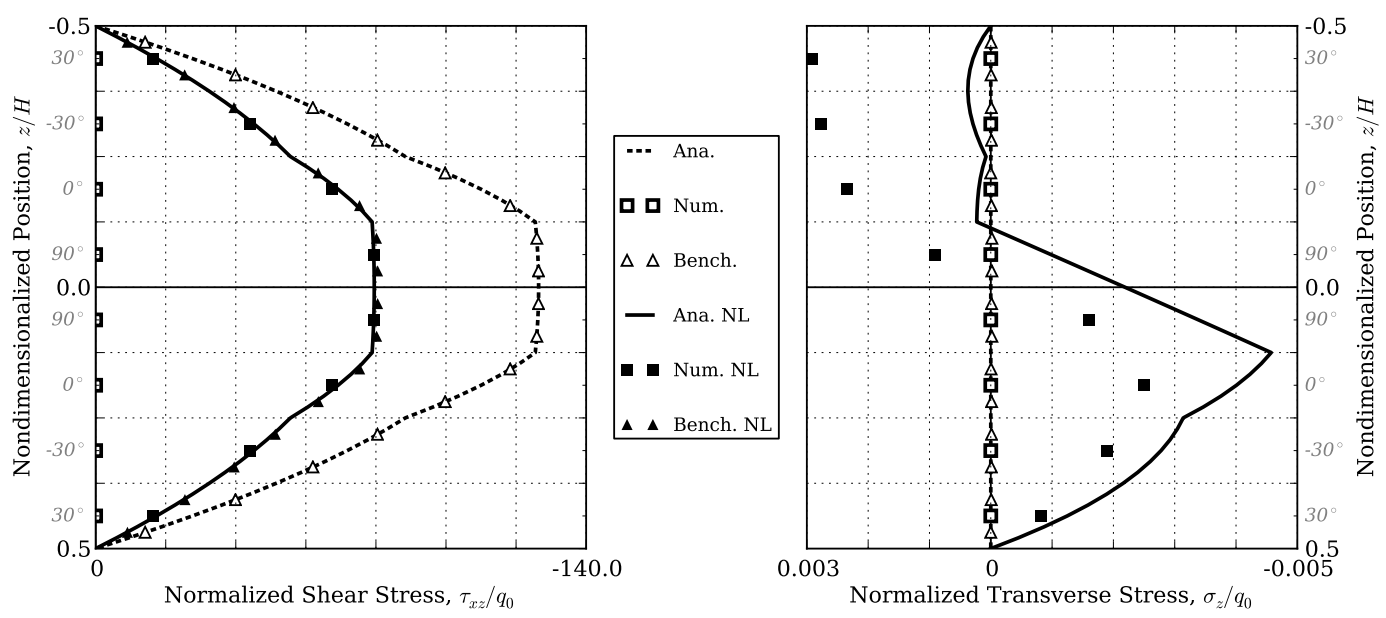

(c) Angle-ply plate.

Figure 4.5: Interlaminar stresses at $x=0.45 \cdot a$ due to transverse loading. 

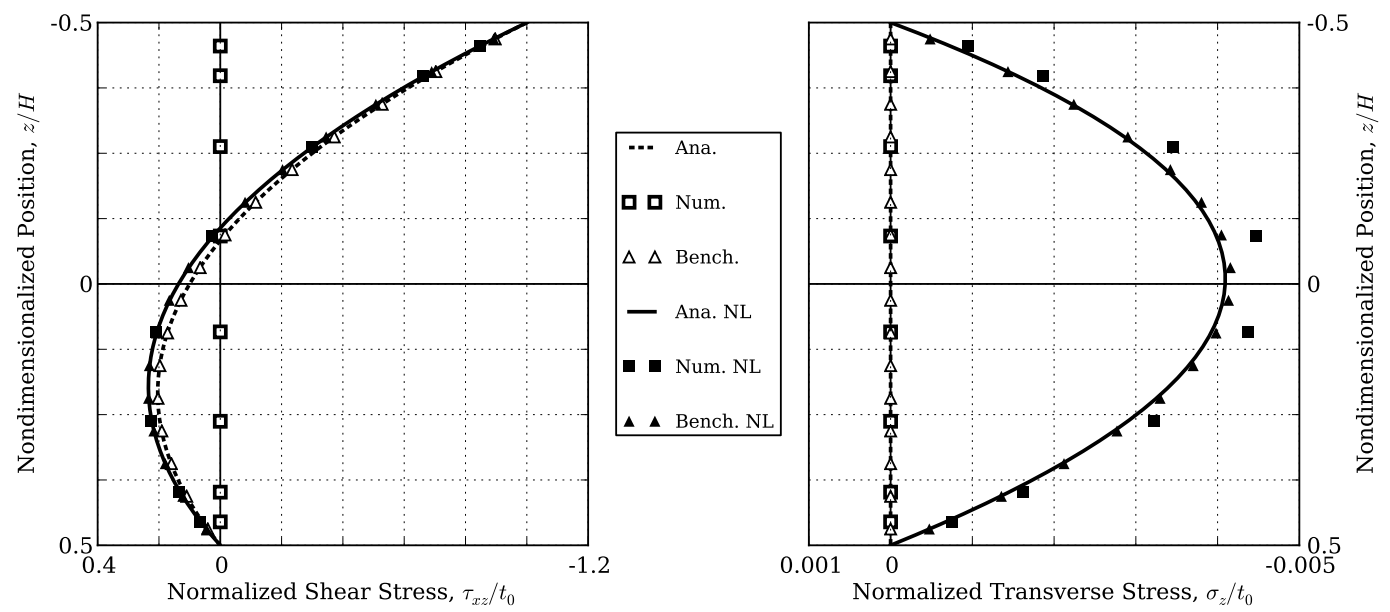

(a) Isotropic plate.
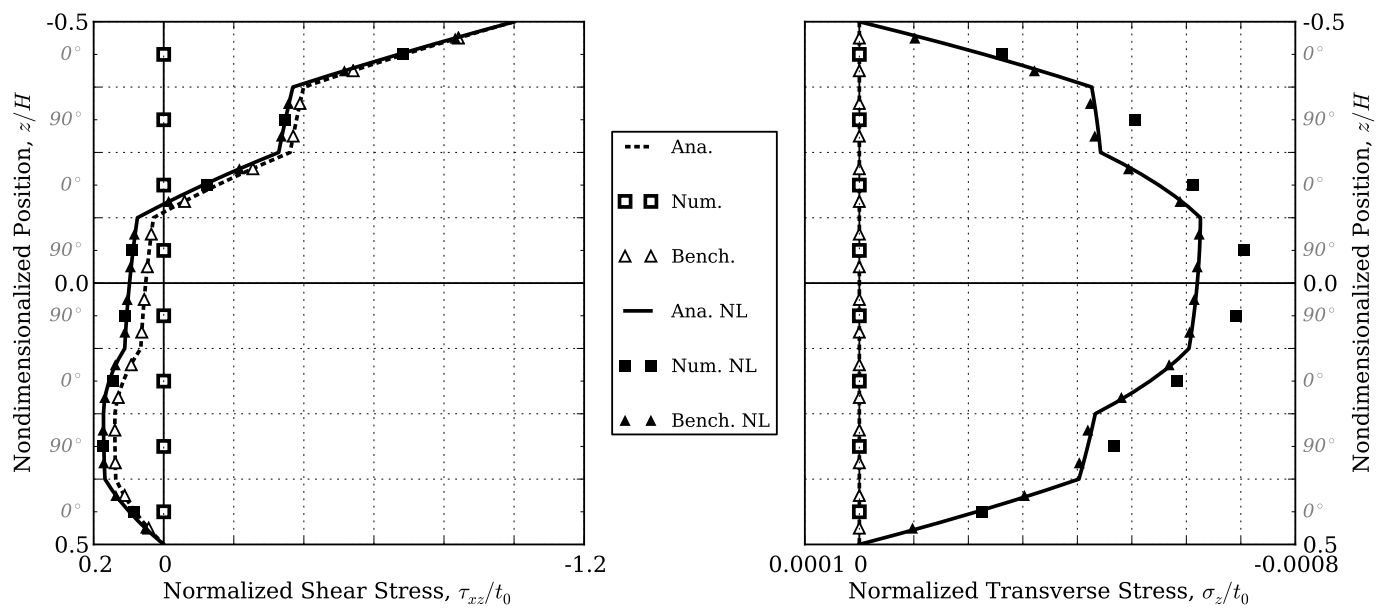

(b) Cross-ply plate.
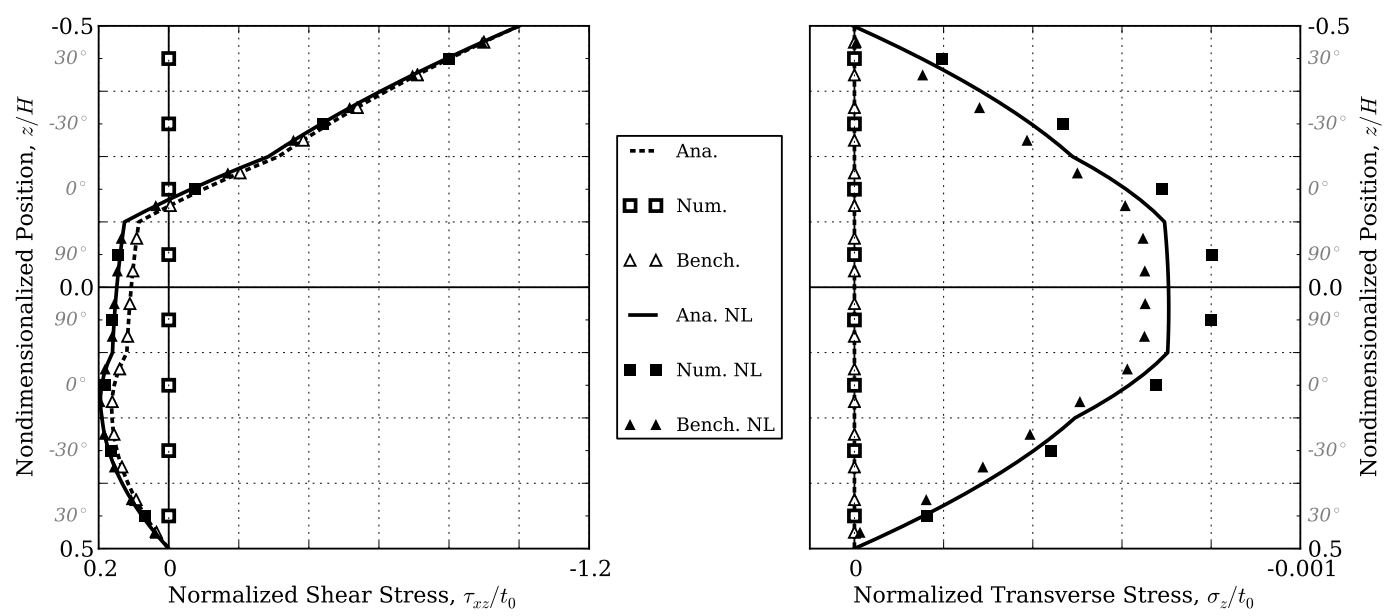

(c) Angle-ply plate.

Figure 4.6: Interlaminar stresses at $x=0.05 \cdot a$ due to shear loading. 
enforced, so the accurate matching of the shear stress boundary condition on the top plate surface is an indication of the accuracy of the developed stress recovery procedure. As seen, for all three plates, using the intralaminar stress results from numerical data in the stress recovery procedure to compute the shear stress distribution matches the distribution from the benchmark three-dimensional finite element results, and also when using the closed-form solution for the intralaminar stresses in the stress recovery procedure. Unlike the transverse load case, the shear stress changes sign within the plate thickness.

Considering the normal stresses in Figure 4.6, which are smaller than the counterpart shear stresses for each particular plate, the unenforced zero stress boundary condition on the top surface is satisfied in each case. For the geometrically linear cases, the normal stresses are identically zero for all three plates. Geometric nonlinearities result in nonzero values for the normal stress within the plate. The small normal stresses for the geometrically nonlinear case exhibit some scatter among the three solutions. For all plates, using the intralaminar stresses from the numerical model in the stress recovery procedure results in stresses that are somewhat different that those of the other two predictions. For the angle-ply case, the benchmark solution does not match the boundary conditions on the top and bottom surfaces. Using the closed-form solution for the intralaminar stresses in the stress recovery procedure agrees with the benchmark solution for the isotropic and cross-ply plates, while differing for the angle-ply plate.

Finally, for the shear loading case, the through-thickness stress distributions for the location near the plate support are illustrated in Figure 4.7. At that location the shear and normal stresses are known to be zero on both the top and bottom surfaces of the plate. Within the scale of the plots, this would appear to be the case for the shear stresses for each of the three plates, but this is not exactly the case for the closed-form solution for the normal stress. For the normal stresses the use of the closed-form solution results in a noticeable error at the top surface of the plate. It should be observed, however, that the normal stresses within the plates are much smaller than the shear stresses, and for the 

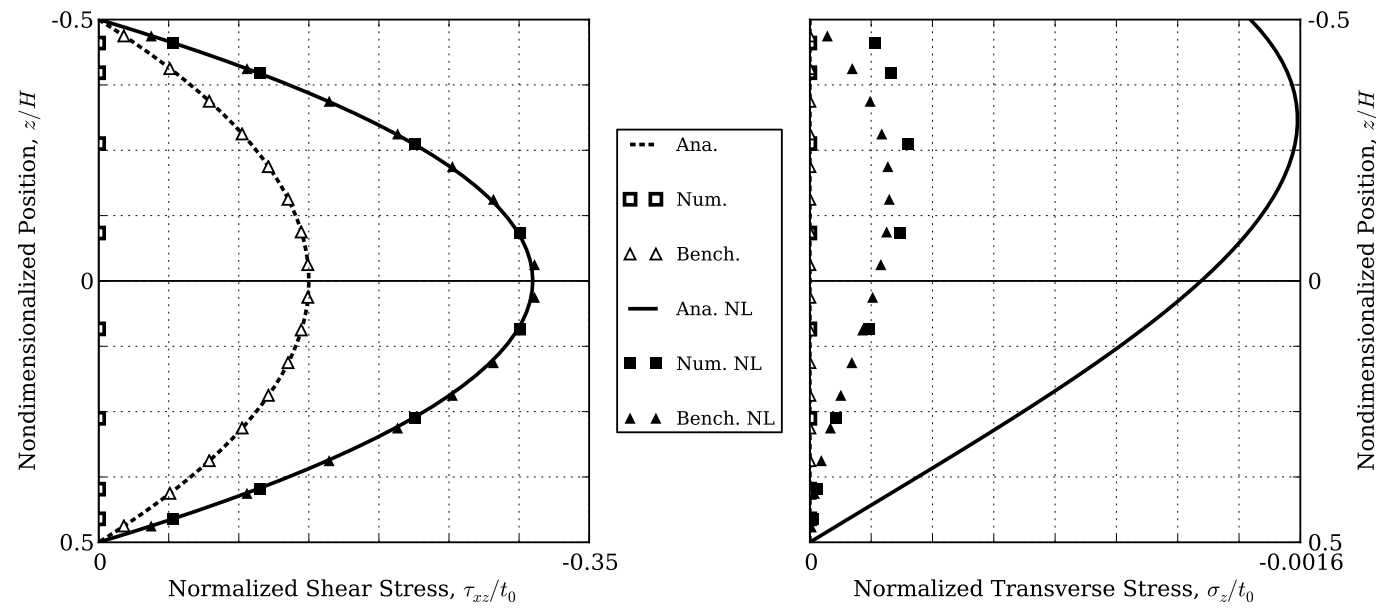

(a) Isotropic plate.
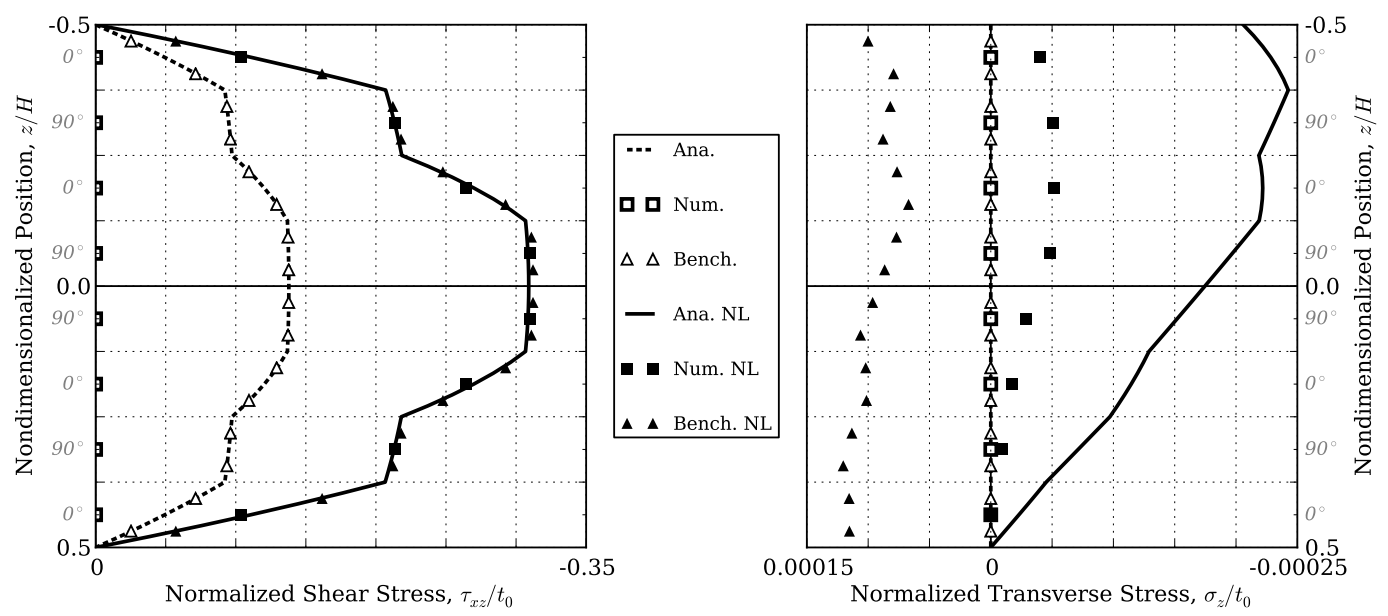

(b) Cross-ply plate.
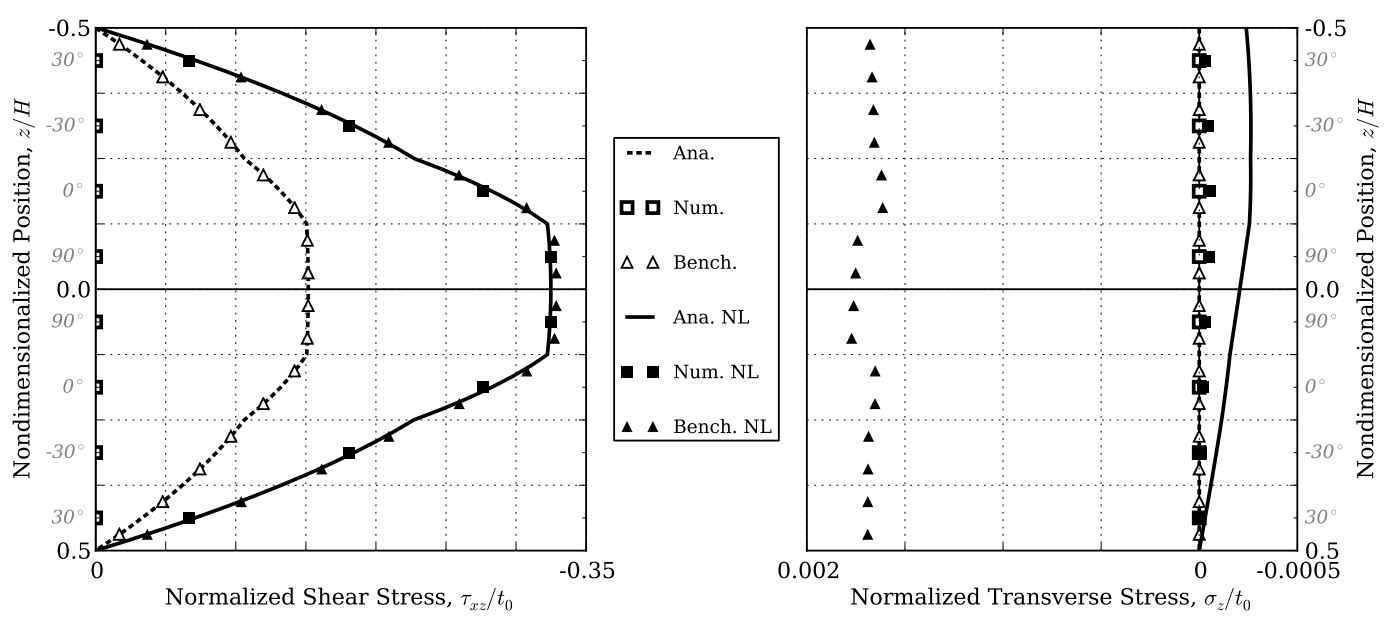

(c) Angle-ply plate.

Figure 4.7: Interlaminar stresses at $x=0.45 \cdot a$ due to shear loading. 
geometrically linear case are identically zero, so the error is of minimal consequence. Considering the details of the shear stresses, use of the intralaminar stresses from the numerical plate model results in interlaminar stresses that compare well with the stresses from the benchmark three-dimensional finite element model and from the closed-form solution. The influence of geometric nonlinearities is correctly represented using intralaminar stresses from the numerical plate model. For the normal stresses, the results from the benchmark model do not match the boundary conditions at the top and bottom surfaces, and there is a shift of those predictions as compared to those of using intralaminar stresses from numerical data in the stress recovery procedure. Again, the normal stresses are so small compared to the shear stresses that comparisons may not be meaningful, as the errors appear to be problem dependent and do not reflect on the generally good results obtained by using intralaminar stresses computed by the numerical plate model in the stress recovery procedure.

\subsection{Discussion and Concluding Remarks}

In this chapter, a straightforward stress recovery procedure was developed by which interlaminar stresses are found by direct use of the intralaminar stresses and cross-section rotations from a plate analysis. The procedure accounts for geometric nonlinearities, and the source of the stress and rotation data may come from any plate solution, though, as stated in the introduction, it is believed that the most important aspect of this paper is the application of the stress recovery procedure to numerical data. Thus, it is important to note that the calculation of the interlaminar stresses is separate from the calculation of the intralaminar stresses and cross-section rotations, so the technique may be applied to any numerical data, including those from a custom or commercial finite element analysis code.

Because the stress recovery procedure involves iteration, numerical integration, finitedifference approximations, and a reliance on finite element data, it is necessary to address the issue of convergence of the results. The iteration procedure converged to within much 
less than one percent by the second iteration $(i=2$ in Eq. (4.5)). Apart from the iteration procedure, convergence of the stress recovery results depends on the accuracy of the rotations and in-plane stresses from the finite element solution, and, in the case of $\sigma_{z z}$, the accuracy of the results of $\tau_{x z}$. Increasing the number of elements across the width of the plate results in more accurate results from the finite element analysis as well as better approximations for gradients using finite-differences. Increasing the number of integration points through the thickness of an element allows for a better representation of $\tau_{x z}$ and may result in a more accurate finite element solution. The convergence metric employed was based on how well the interlaminar normal stress matched the known traction on the top surface of the plate. As the interlaminar normal stress calculation depends upon finite-difference approximations in and numerical integration of two equilibrium equations, as well as the calculated value of the interlaminar shear stress from the first integration, using the normal stress as a metric is appropriate. Considering the isotropic, transversely loaded plate, using eight throughthickness integration points and 10 elements across the width resulted in $\sigma_{z z}$ at the top surface that was $32.3 \%$ less than the known boundary condition. Increasing the number of elements across the width to 30 gave a result $6.67 \%$ less. Increasing to 50 elements (the number used for the results shown) gave a $\sigma_{z z} 1.25 \%$ less. Similarly, using 50 elements but decreasing the number of through-thickness integration points to six increased the error to a value $1.96 \%$ less than the known stress. Further reducing to four integration points gave a result $4.23 \%$ less than the known traction boundary condition.

From the results presented, it is clear that the objective of developing a stress recovery procedure based on a finite element plate model and the geometrically nonlinear equilibrium equations of the theory of elasticity was accomplished. The procedure was applied to two example plate problems with consideration of isotropic and composite materials. One example problem, the transverse loading case, exhibited geometrically nonlinear effects in the form of membrane forces, while the other example problem, the shear loading case, exhibited geometrically nonlinear effects because the shear loading was considered to be a follower force, 
and membrane effects were also present. In each case the loading acted on only a portion of the plate. With two different loading cases, isotropic and composite materials, and the loading on only a portion of the plate, a variety of stress responses was examined. Additionally, there was a variety of traction conditions on the top surface of the plate. Except when one interlaminar stress component was very small compared to the other, the results from the developed stress recovery procedure were quite accurate. This statement of accuracy is supported by the good correlation with results obtained from the benchmark three-dimensional finite-element model, and from using closed-form plate solutions in the stress recovery procedure. This good correlation included both the distribution of the interlaminar stresses through the plate thickness, and the matching of traction boundary conditions on the top surface of the plate. Cases in which a recovered stress was orders of magnitude less than the other recovered stresses and lacked correlation to the known traction boundary condition are considered an issue.

From the discussion of the stress recovery procedure it is clear how the procedure can be extended to finite plates. The necessary stresses and rotations in the equilibrium equations dependent on the $y$-direction are available from a plate finite element analysis. The iteration procedure would then include these additional data. 


\section{Chapter 5}

\section{Stress Recovery in a Finite Domain}

Results from this section were presented [56] as:

T. B. Hartman, M. W. Hyer, and S. W. Case (Oct. 2012b). Geometrically Nonlinear Stress Recover in Composite Laminates Considering Inertial Effects. In: American Society for Composites 27th Technical Conference. Arlington, TX

The example problem of Chapter 4 involving a semi-infinite plate is now extended to a finite plate, where all three transverse stresses are to be found. Thus, rather than examining only a cross-section of a plate, the entire two-dimensional domain of the plate is available for analysis. No closed-form or semi-closed form solutions are used in the stress recovery procedure, rather only plate finite element stress and rotation data are used. A threedimensional finite element solution is again used as a benchmark.

To limit the scope of the problem, only the $\left[0^{\circ} / 90^{\circ}\right]_{2 S}$ cross-ply plate of Section 4.3.1 is examined. Properties of this plate remain unchanged, as given in Tables 4.2 and 4.3. Recall that the thickness of the eight-layer plate is $1.2 \mathrm{~mm}$.

Consider a square plate of the $\left[0^{\circ} / 90^{\circ}\right]_{2 S}$ cross-ply laminate, simply supported along all four edges, as shown in Figure 5.1. The simple supports restrict translation in all three directions, but allow rotation about the edges of the plate. The boundary conditions at the 


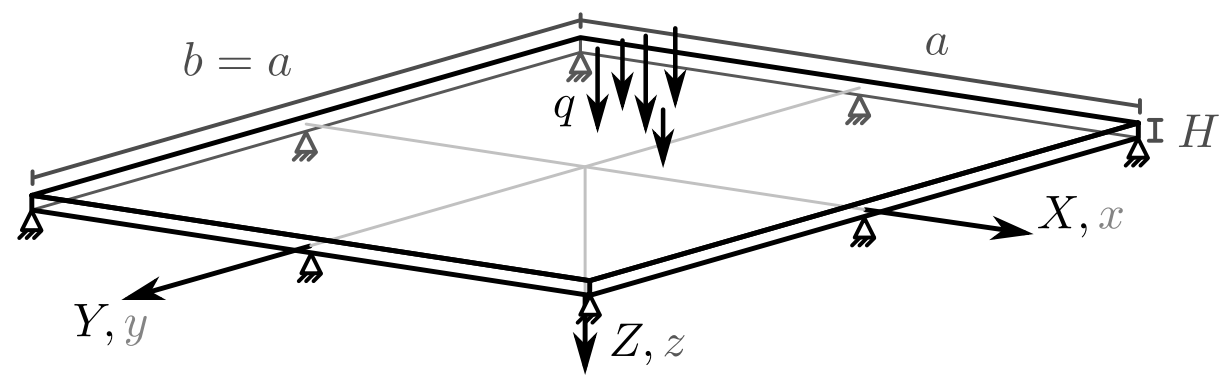

Figure 5.1: Simply supported plate.

$\pm x$-edges for the finite element plate analysis are given by

$$
\begin{array}{lll}
\left.u^{\circ}\right|_{x= \pm a / 2}=0 & \left.v^{\circ}\right|_{x= \pm a / 2}=0 & \left.w^{\circ}\right|_{x= \pm a / 2}=0 \\
\left.\omega_{x}\right|_{x= \pm a / 2}=0 & \left.M_{y}\right|_{x= \pm a / 2}=0 & \left.\omega_{z}\right|_{x= \pm a / 2}=0
\end{array}
$$

where $u^{\circ}, v^{\circ}$, and $w^{\circ}$ are the $x$-, $y$-, and $z$-displacements, respectively, at the plate reference surface. Those at the $\pm y$-edges are given by

$$
\begin{array}{lll}
\left.u^{\circ}\right|_{y= \pm b / 2}=0 & \left.v^{\circ}\right|_{y= \pm b / 2}=0 & \left.w^{\circ}\right|_{y= \pm b / 2}=0 \\
\left.M_{y}\right|_{y= \pm b / 2}=0 & \left.\omega_{y}\right|_{y= \pm b / 2}=0 & \left.\omega_{z}\right|_{y= \pm b / 2}=0
\end{array}
$$

The plate is loaded with a distributed load $q(x, y)$, shown in Figure 5.1, on the top surface. Unless otherwise stated, the distributed load will be a uniformly distributed live load such that the load follows the rotations of the plate (a change from the transverse dead load of Chapter 4).

$$
q(x, y)=q_{0}
$$

As such, the tractions at the top surface $(z=-H / 2)$ of the plate will be everywhere given by

$$
\tau_{x z}=0 \quad \tau_{y z}=0 \quad \sigma_{z z}=q_{0}
$$




\subsection{Description of Solution Methods}

As mentioned, the response of the square plate to a distributed pressure load is predicted using two methods. A plate finite element solution provides discretized data for use in the geometrically nonlinear stress recovery procedure, which is used to obtain estimations of the three transverse stresses. A three-dimensional finite element solution is also obtained and used as the benchmark to which the recovered stresses will be compared. Both finite element solutions are implemented in the commercial finite element code LS-DYNA [54], and share several common features.

Symmetry boundary conditions are employed in each model so that only a quarter of the plate $(0<x<a / 2,0<y<b / 2)$ is modeled. The symmetry boundary condition on the $x=0$ edge is applied by restricting displacement in the $x$-direction and, for the plate model, rotations about the $y$ - and $z$-axes. The symmetry boundary condition on the $y=0$ edge is applied by restricting displacement in the $y$-direction and, for the plate model, rotations about the $x$ - and $z$-axes.

Material properties from Table 4.2 are input to the linear elastic orthotropic material model (*MAT_ORTHOTROPIC_ELASTIC or *MAT_002). LS-DYNA's implicit solver is used, as the example problem is quasi-static, and automatic timestep control is employed, which allows as many solution steps to be used as are needed for convergence of the solution. Both the plate and three-dimensional finite element solutions are geometrically nonlinear, the LS-DYNA default solution technique.

\subsubsection{Finite Element Plate Solution}

A uniform mesh of four-noded constant-strain shell elements (LS-DYNA type 2; BelytschkoTsay) models the $0<x<a / 2,0<y<b / 2$ quarter domain of the plate. All elements are square and initially aligned with the $X-Y-Z$ global coordinate system. The Belytschko-Tsay element is a shear deformable element, with the default shear correction factor of 1.0 used 
for all analyses. The load is applied as a pressure load to each element, the value of which is $q(x, y)$ of Eq. (5.3) evaluated at the element centroid.

The simply supported edges at $x= \pm a / 2$ and $y= \pm b / 2$ are constrained from displacement and rotation according to Eqs. (5.1) and (5.2).

Unless otherwise specified, a mesh of 55 elements by 55 elements (total: 3025 ) is used with two integration points per physical layer for a total of 16 integration points through the thickness located at the centroid of each element. The effect of mesh density and number of integration points on the solutions will be discussed.

\subsubsection{Finite Element Benchmark 3D Solution}

The benchmark solution, to which the recovered transverse stresses will be compared, consists of a uniform mesh of eight-noded constant-strain solid elements (LS-DYNA type 1), with a single integration point located at the centroid of each element. The mesh is a discretization of the $0 \leq x \leq a / 2,0 \leq y \leq b / 2,-H / 2 \leq z \leq H / 2$ quarter domain of the plate.

Nodes on the $x=0$ plane of symmetry are constrained from translation in the $x$-direction, while nodes on the $y=0$ plane are constrained from translation in the $y$-direction. Because each node only has translational degrees of freedom, no constraints are imposed on the rotations. All elements are equally-sized right rectangular prisms with square faces initially parallel to the $X-Y-Z$ global coordinate system so that the numbers of elements along the $x$ and $y$ edges are equal. To model the eight-layered laminate, a minimum of one element per physical layer is required. To refine the mesh in the thickness dimension, an equal number of elements is used per each physical layer so that element size through the thickness is one of $H / 8, H / 16, H / 24$, etc. The effect of mesh density both in the $x$ - $y$ plane and through the thickness will be discussed.

A difficulty in comparing the plate solution to the benchmark three-dimensional solution is in the application of boundary conditions at the simply supported edges in the threedimensional solution. Application of the zero-displacement boundary condition to the nodes 
at the reference surface $(z=0)$ alone creates severe force discontinuities which not only cause an overly compliant response, but also cause difficulties in convergence near the simply supported edges. In seeking to avoid the problems associated with the force discontinuities, and to realistically model the type of response that is indicated by a simply-supported edge, the following method of constraint application is applied to the simply-supported edges of the solid model.

For an eight-layered solid model with two numerical layers per physical layer, consider the set of nodes located at $x=a / 2, y=0$, as shown in Figure 5.2a. In modeling this $x^{+}$ simply-supported edge, the node located at the reference surface (labeled $n=0$ ) is fixed from translation in all directions. To distribute the resultant force at this node as a result of these translation constraints, the other nodes are constrained as well. Using the notation described in Figure 5.2a, with the second node away from the reference surface node in the $-z$-direction labeled as Node $n=-2$, the following constraints are applied via LS-DYNA's *CONSTRAINT_LINEAR_GLOBAL keyword:

$$
\begin{aligned}
& 0=u^{n}+u^{-n} \\
& 0=v^{n}+v^{-n} \\
& 0=w^{n}+w^{-n}
\end{aligned}
$$

with $n$ ranging from one to eight for the laminate represented in Figure 5.2a. In Eq. (5.5), $u^{n}$ is the displacement in the global $X$-direction of the $n^{\text {th }}$ node, etc. For a plate with a spanratio $a / H$ of 60 and a normalized center deflection $w / H$ of unity, the resulting deformation is shown in Figure 5.2b. The shear-deformable model assumes the deformed edge remains straight. The deviation of the nodal positions of Figure 5.2b from a straight line (calculated by a least-squares fit of the deformed coordinates) is shown in Figure 5.2c. The deviation is very small, with a maximum value of about $5 \times 10^{-5}$ of a plate thickness. 


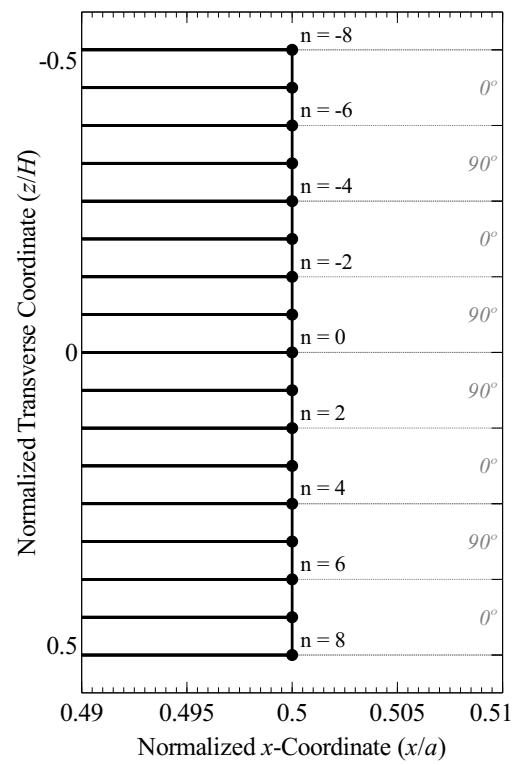

(a) Before deformation.

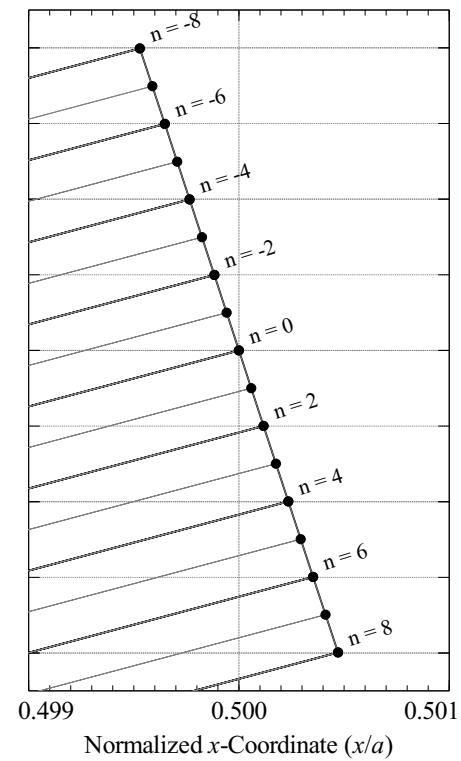

(b) After deformation.

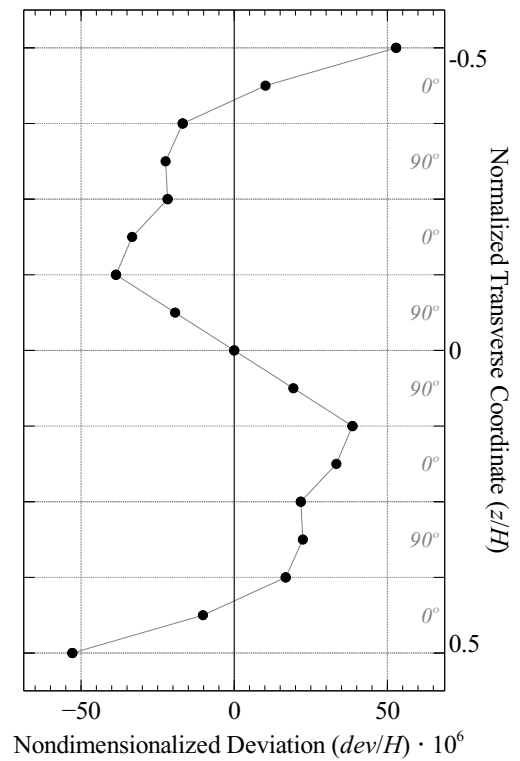

(c) Deviation from linear.

Figure 5.2: Deformation of nodes through the thickness at $x=a / 2, y=0$ due to enforced boundary condition.

\subsection{Results}

\subsubsection{Summary of Plate Response}

For a plate with span-ratio $a / H=60$, the relationship between the center deflection and applied uniform pressure load, as determined by the LS-DYNA plate model, is shown in Figure 5.3 for a variety of mesh densities. With only 15 plate elements per edge of the quarter model, the center displacement is converged to within $0.3 \%$ of results given by more refined meshes. As a representation of the magnitude of the deformations of this plate, Figure 5.4 shows a cross-section of the quarter model at $y=0$, with all plate dimensions and deformations shown to scale.

Considering now the plate response at a central plate deflection of one plate thickness $(w / H=1)$, the intralaminar stresses at the center of the quarter model $(x / a=0.25$, $y / b=0.25)$, as computed by LS-DYNA, are shown in Figure 5.5. The distribution of 


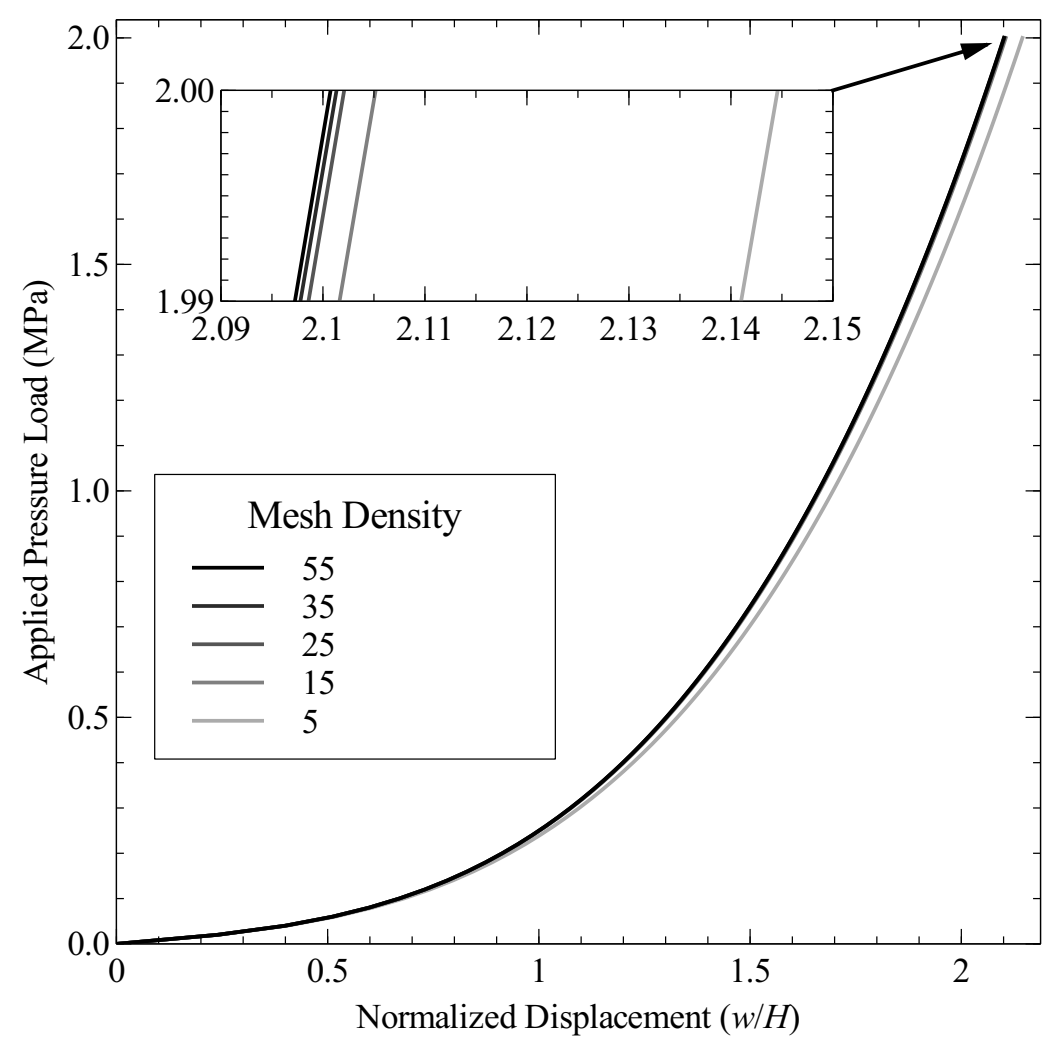

Figure 5.3: Force-displacement relationship for $a / H=60$ with varying mesh densities (elements along edge) of plate finite element model.

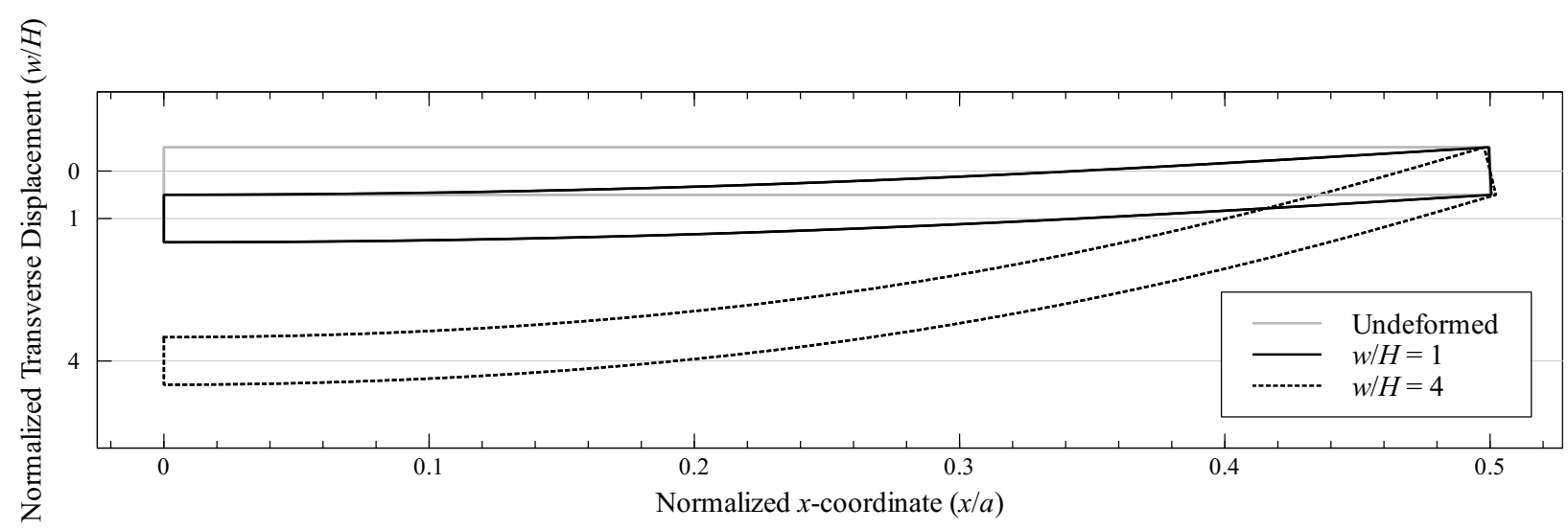

Figure 5.4: Plate displacement, to scale.

the in-plane normal stress $\sigma_{x x}$ through the thickness is shown in Figure 5.5a for the threedimensional benchmark analysis (solid circles), as well as varying mesh densities for the plate analysis. Note that the stresses for the plate analysis are shown considering the piecewise 
linear intra-layer interpolation scheme presented in Section 3.4.4. Even with the coarse five-by-five mesh, $\sigma_{x x}$ is within $1.5 \%$ of the converged two-dimensional solution.

The intralaminar normal stress $\sigma_{y y}$ and shear stress $\tau_{x y}$ are shown in Figures 5.5b and 5.5c, respectively. In these plots, the stresses as calculated by the three-dimensional benchmark solution are again shown as solid circles. Three sets of data are shown as calculated by the plate analysis using a mesh with 55 elements per edge (total: 3025). The solid line again shows the solution using the piecewise linear intra-layer interpolation scheme. Additionally, two data sets are shown for a plate analysis using one integration point per layer (eight total integration points), and four integration points per layer (32 total integration points). For these latter two data sets, the distribution is shown as piecewise constant through each numerical layer, as given directly by the plate finite element analysis, and as discussed in Section 3.4.3. Note that the interpolation scheme appropriately represents the distribution of stresses through the thickness, as compared to both the three-dimensional solution, as well as the trend of the plate analysis when increasing the number of integration points.

As mentioned, for the results to be shown, the benchmark three-dimensional finite element simulation was made with 55 elements along the $x$ - and $y$-edges of the plate and 24 elements through the thickness of the plate (three elements per physical layer). As shown in Figure 5.6, the intralaminar stresses are converged with a mesh of 25 elements per side and eight elements through the thickness $(25 \times 25 \times 8)$. However, in order to resolve the smaller transverse stresses, a more refined mesh is required. As shown in Figure 5.7, while the transverse normal stress $\sigma_{z z}$ is converged with the refined $55 \times 55 \times 8$ mesh, the transverse shear stresses require 24 elements through the thickness (particularly $\tau_{y z}$ ) before a converged solution is obtained. Note, especially, the unmet traction boundary conditions $\left(\tau_{y z}=0\right)$ at the top and bottom surface for the transverse shear stress $\tau_{y z}$. In order to be able to compare stress data from the stress recovery method, the refined mesh of 55 elements per edge and 24 elements through the thickness is used for all results shown herein. 


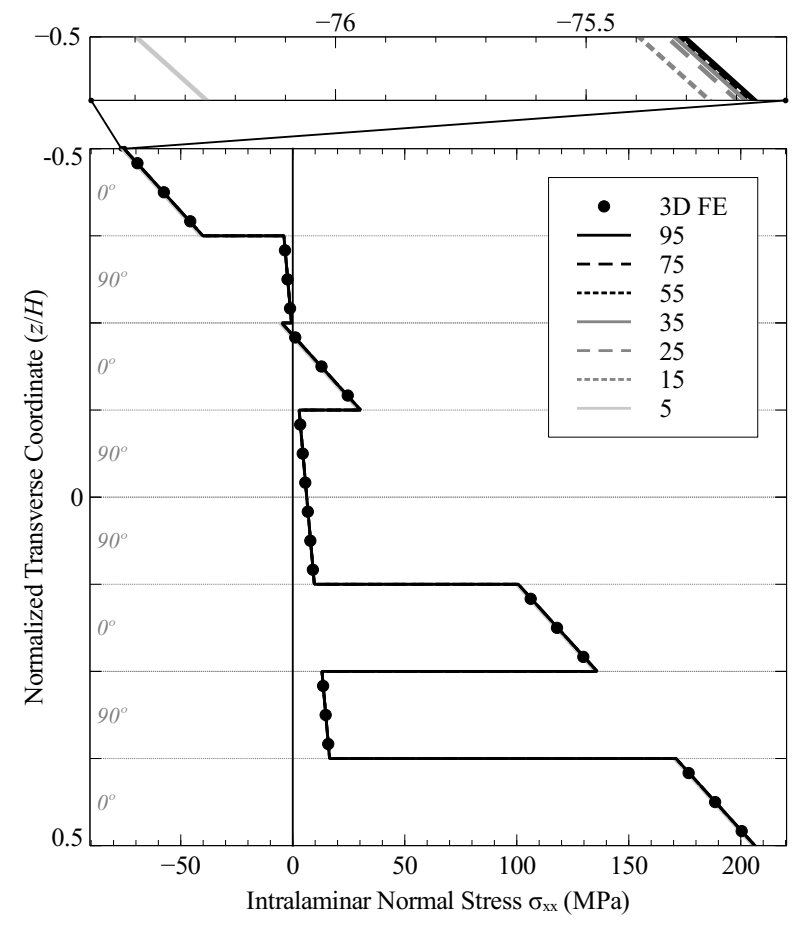

(a) Intralaminar normal stress $\sigma_{x x}$.

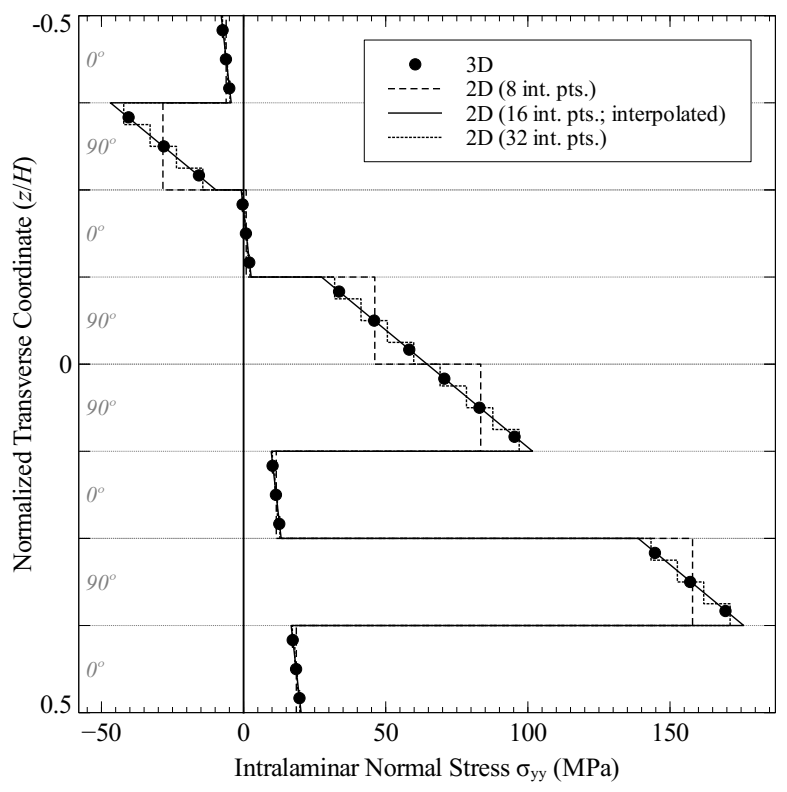

(b) Intralaminar normal stress $\sigma_{y y}$.

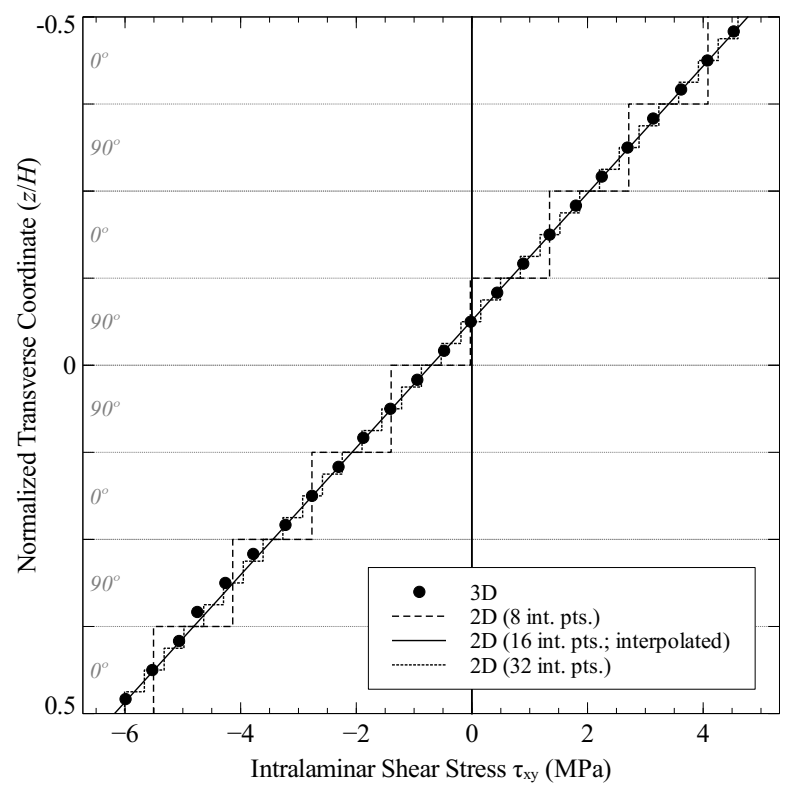

(c) Intralaminar shear stress $\tau_{x y}$.

Figure 5.5: Intralaminar stress distribution at center of quarter model $(x / a=0.25, y / b=$ $0.25)$. 


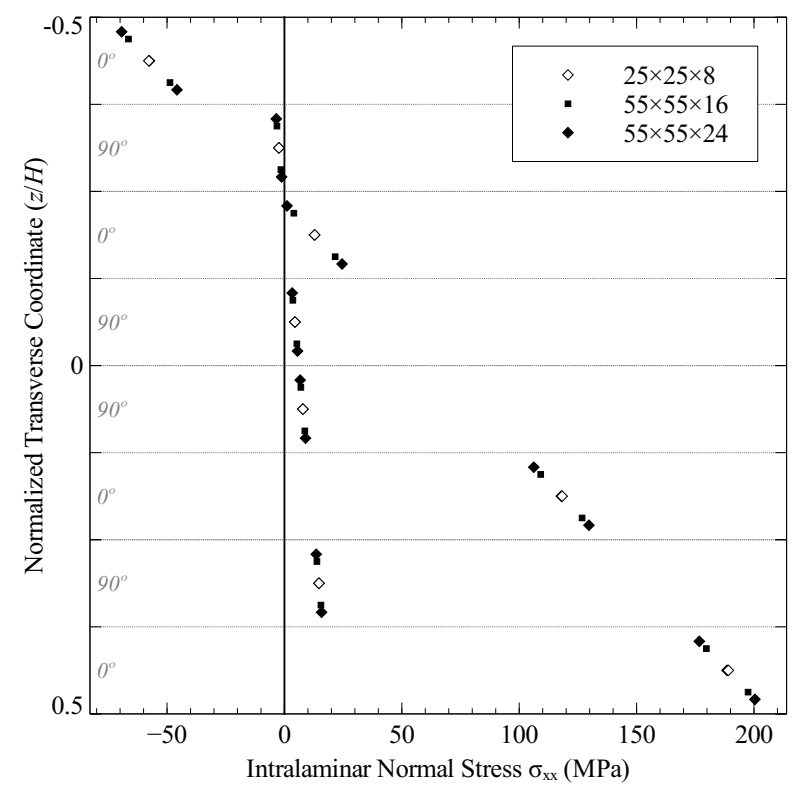

(a) Intralaminar normal stress $\sigma_{x x}$.

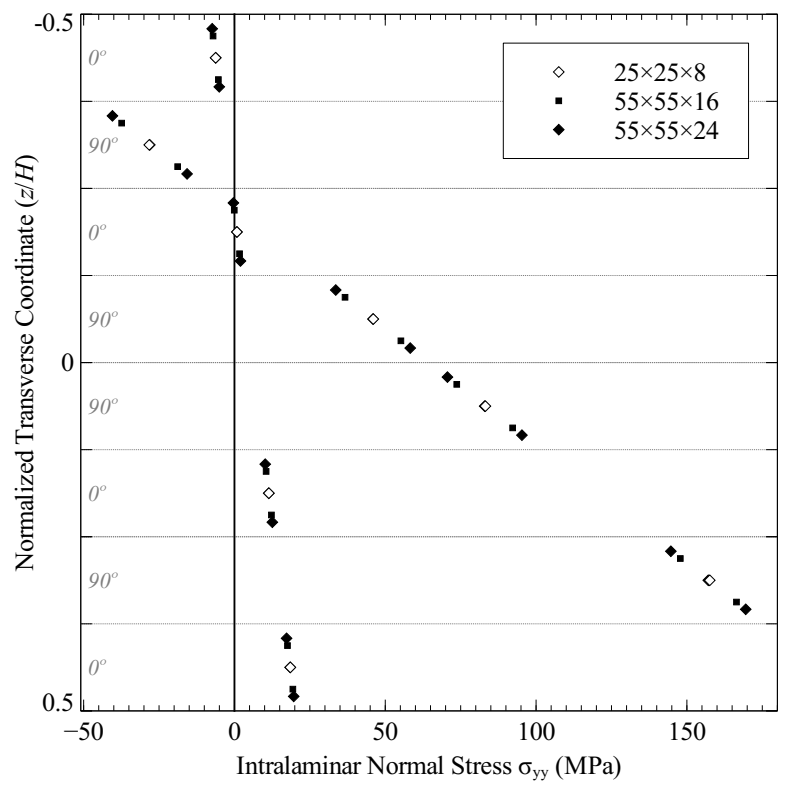

(b) Intralaminar normal stress $\sigma_{y y}$.

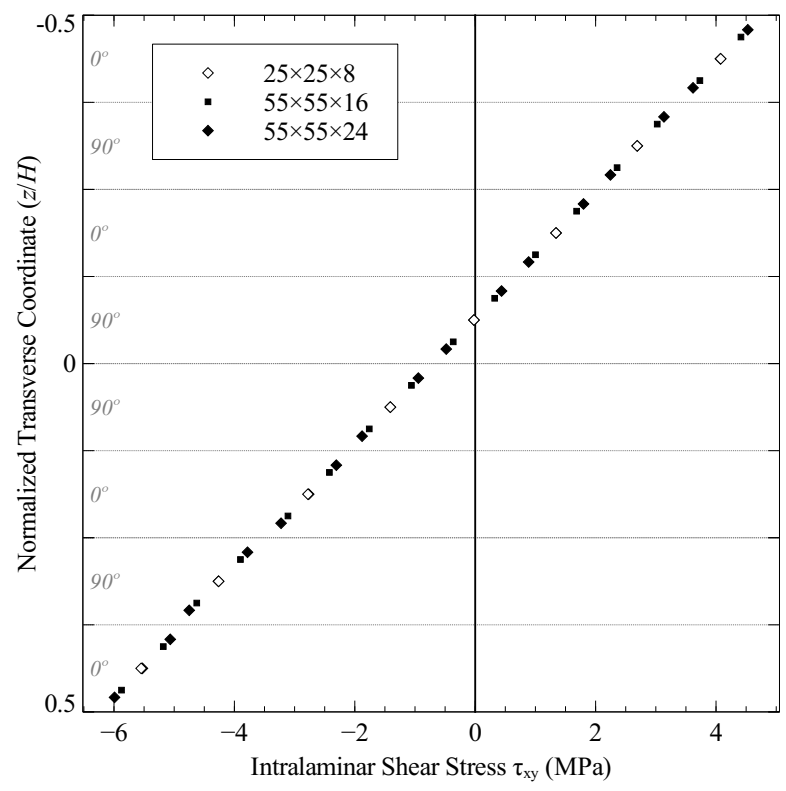

(c) Intralaminar shear stress $\tau_{x y}$.

Figure 5.6: Convergence of intralaminar stresses for benchmark solution at center of quarter model $(x / a=0.25, y / b=0.25)$. 


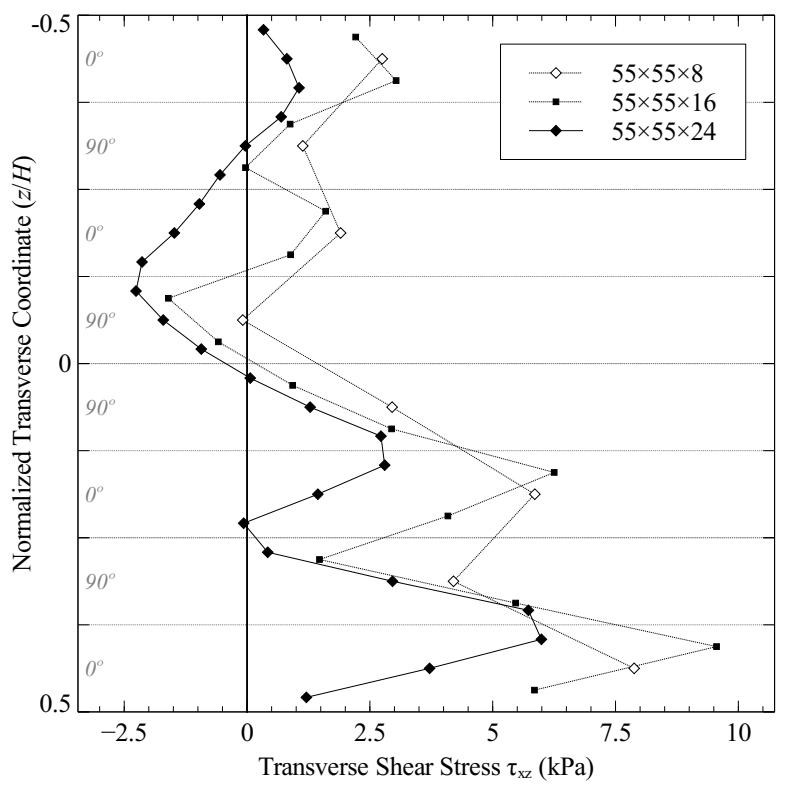

(a) Transverse shear stress $\tau_{x z}$.

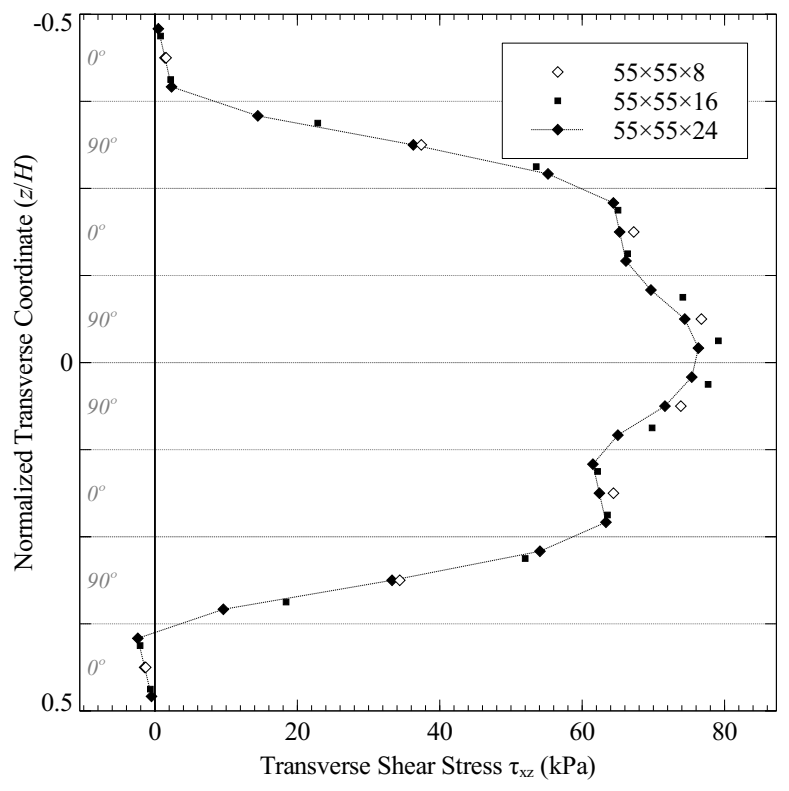

(b) Transverse shear stress $\tau_{y z}$.

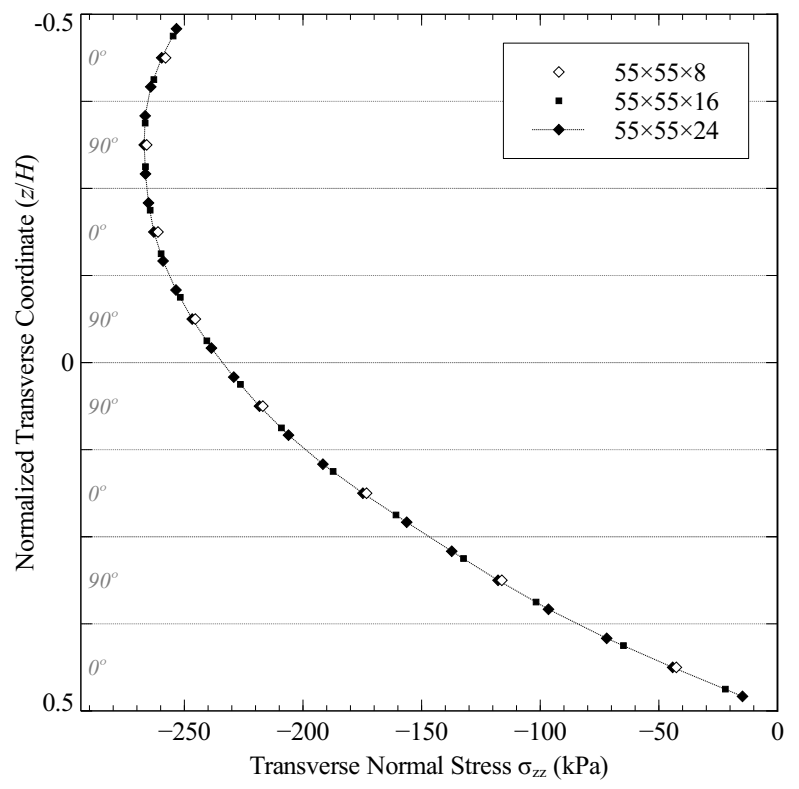

(c) Transverse normal stress $\sigma_{z z}$.

Figure 5.7: Convergence of tranverse stresses convergence for benchmark solution near plate center. 


\subsubsection{Recovery of Transverse Stresses}

In order to investigate the accuracy of the geometrically nonlinear stress recovery procedure, transverse stresses are recovered at four locations in the quarter plate model, shown in Figure 5.8, each which experience a unique state of stress. While the traction at the top surface is identical everywhere on the plate, the relative magnitude of the transverse shear stresses is known to vary within the plate. Point A lies at the center of the quarter model and will be termed the quarter-point. Data in plots which describe the response at the quarter-point will be depicted by the filled circle "๑". At this point, the magnitude of each transverse shear stress is similar and is moderate compared to other locations in the plate. As will be shown, the magnitudes of the transverse shear stresses are approximately equal to that of the transverse normal stress at this point. Point B lies near the center of the plate, four elements in from either edge (that is, at the centroid of the element with three elements between itself and the edge of the quarter model). The reason for this particular location will be described shortly. At Point B, the magnitudes of the transverse shear stresses are small compared to the transverse normal stress. (At the center of the plate, both transverse

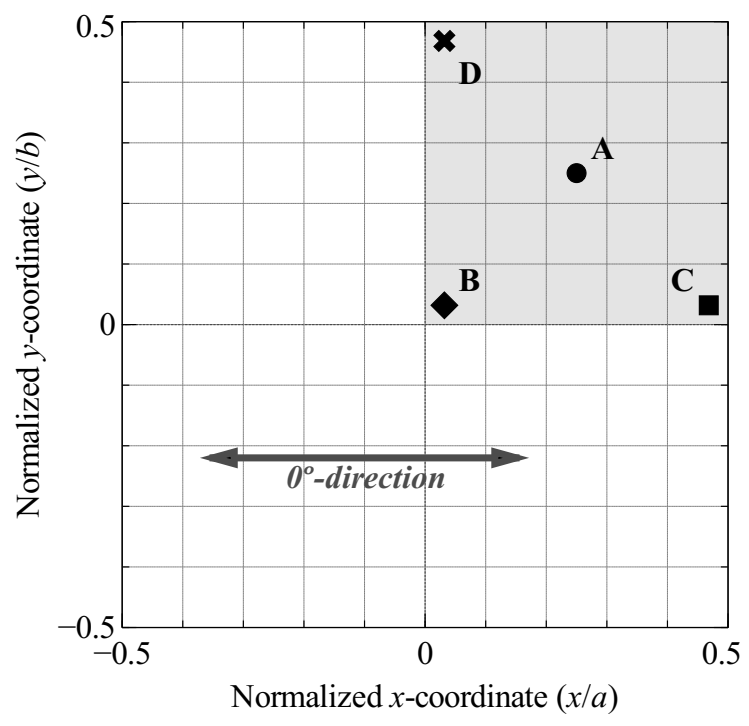

Figure 5.8: Locations of stress recovery results. 
shear stresses are identically zero.) Data for this location will be shown as the filled diamond

Points $\mathrm{C}$ and $\mathrm{D}$ lie near the center of the supported $x^{+}$- and $y^{+}$-edges of the plate, respectively. At Point $\mathrm{C}$, the transverse shear stress $\tau_{x z}$ will be large compared to the transverse normal stress, while the transverse shear stress $\tau_{y z}$ will be nearly zero, as it is near the $y=0$ plane of symmetry. Data from Point $\mathrm{C}$ will be shown as filled squares "⿴囗十". At Point $\mathrm{D}$ this relationship is reversed $-\tau_{y z}$ will be large while $\tau_{x z}$ will be nearly zero. Data from Point D will be shown as filled crosses "*." Like Point B, Points C and D lie four elements away from the edges of the plate. Table 5.1 summarizes the locations and magnitudes of stresses at each of these points.

As mentioned, Points B, C, and D all lie four elements from the edges of the plate. The current implementation of the stress recovery procedure uses the central difference method for calculating numerical derivatives of stresses required by Eq. (3.14). As such, data are required from elements bordering the element for which stresses are to be recovered. If two iterations of the stress recovery procedure are required, data are required from elements bordering the set of bordering elements used for the first iteration. Thus, for the $n^{\text {th }}$ iteration of the stress recovery procedure, data is required from elements that are $n$ elements away from the location where stresses are to be recovered.

This requirement may be shown graphically by considering Figure 5.9. A mesh of 49 square elements is shown. In Figure 5.9a, elements are highlighted to which the stress recovery procedure may be applied. Note that the elements bordering the edge of the mesh

Table 5.1: Summary of the properties of examined plate locations.

\begin{tabular}{cccccc}
\hline \multirow{2}{*}{ Point } & \multirow{2}{*}{ Plot Symbol } & \multicolumn{2}{c}{ Coordinate } & \multicolumn{2}{c}{ Stress Magnitude } \\
& & $x / a$ & $y / b$ & $\tau_{x z}$ & $\tau_{y z}$ \\
\hline A & $\bullet$ & 0.25000 & 0.25000 & moderate & moderate \\
B & $\bullet$ & 0.03182 & 0.03182 & small & small \\
C & $\mathbf{Q}$ & 0.46818 & 0.03182 & large & small \\
D & $\boldsymbol{*}$ & 0.03182 & 0.46818 & small & large \\
\hline
\end{tabular}




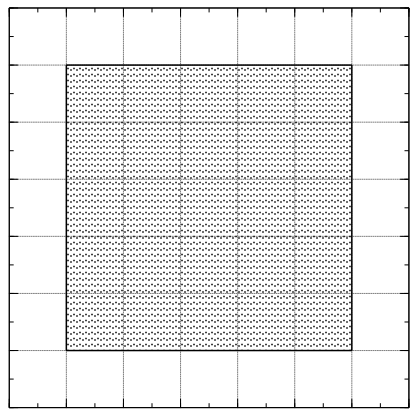

(a) First iteration.

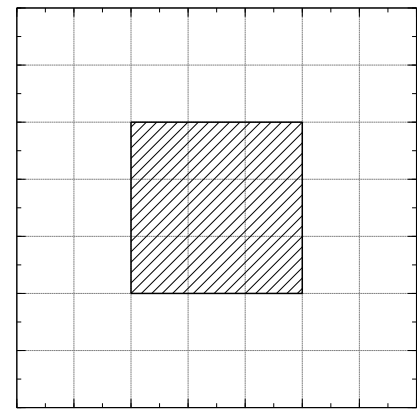

(b) Second iteration.

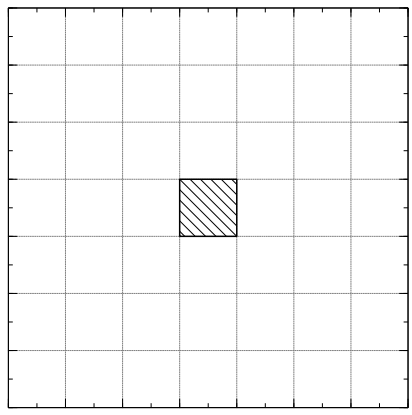

(c) Third iteration.

Figure 5.9: Graphical representation of elements involved in iteration procedure.

are not involved in the stress recovery, as stress gradients may not be found by the central difference method described in Section 3.4.1. After one iteration of the stress recovery procedure, the transverse stresses of the highlighted elements, and only the highlighted elements, in Figure 5.9a would be updated according to the stress recovery technique. In order to use stress data from the first iteration of the stress recovery procedure, the number of elements to which the stress recovery procedure may be applied in a second iteration is depicted in Figure 5.9b. Likewise, as shown in Figure 5.9c, only the center element of the 49-element mesh may be used for the third iteration of the stress recovery procedure. That is, in the $n^{\text {th }}$ iteration of the stress recovery procedure, data is required from elements that are $n$ elements away from the location where stresses are to be recovered.

It will be shown that the accurate recovery of the transverse normal stress requires two iterations of the procedure, so transverse stresses may be recovered accurately at elements which are at least two elements away from the edge. Additionally, it will be shown that the recovered stresses are inaccurate in the first three elements near the supported edges. Thus, distributions for recovered stresses are shown here for the fourth elements in from the edge.

The results of the stress recovery shown below are given for a plate with span-ratio $a / H=60$ and normalized center plate deflection $w / H=1$. A mesh of 55 elements per side with two integration points per layer (16 integration points total) is used and the piecewise linear intralaminar interpolation technique of Section 3.4.4 is employed. The result- 
ing stress distributions are normalized by the applied upper surface $(z=-H / 2)$ traction, $q_{0}=-250 \mathrm{kPa}$. The benchmark solution is given by the three-dimensional solution with 55 elements in the $x$ - and $y$-directions of the plate, with three elements per layer ( 24 elements through the thickness).

\subsubsection{Recovered Stresses at Quarter-Point, Point A}

Results for the recovery of the transverse stresses at the quarter-point, Point A, are shown in Figure 5.10. Consider first the recovery of the transverse shear stress $\tau_{x z}$, given in Figure 5.10a. Stresses from the three-dimensional benchmark solution are given as discrete points shown as solid circles "๑". (Note the horizontal stress axis is normalized by the known normal traction at the top surface, $q_{0}=-250 \mathrm{kPa}$. While the largest magnitude of the shear stress is negative, its normalized value is positive.) The recovered $\tau_{x z}$ from the geometrically nonlinear stress recovery procedure discussed herein is shown as the solid line. For comparison, the results of recovering the transverse shear stress $\tau_{x z}$ via the geometrically linear stress recovery procedure are shown as the dashed line. The difference in the geometrically linear procedure is that all terms of Eqs. (3.5) and (3.23) containing the rotations are neglected (that is, $\omega_{x}=\omega_{y}=\omega_{z}=0$ ).

Results of the recovery of $\tau_{x z}$ from both the geometrically linear and geometrically nonlinear procedures show good agreement with the stresses from the benchmark solution, with the geometrically linear procedure showing slightly better agreement.

The results of the recovery of the second transverse shear stress $\tau_{y z}$ are shown in Figure 5.10b, with the same sets of data as in Figure 5.10a. In this case, the benchmark solution is clearly more in agreement with the geometrically linear procedure than the geometrically nonlinear procedure. Additionally, $\tau_{y z}$ from the geometrically linear procedure matches the known shear traction $\left(\tau_{y z}=0\right)$ at the top surface of the laminate $(z / H=-0.5)$, while that of the geometrically nonlinear procedure does not match that traction boundary condition.

The results of the recovery of the transverse normal stress $\sigma_{z z}$ are shown in Figure 5.10c, 


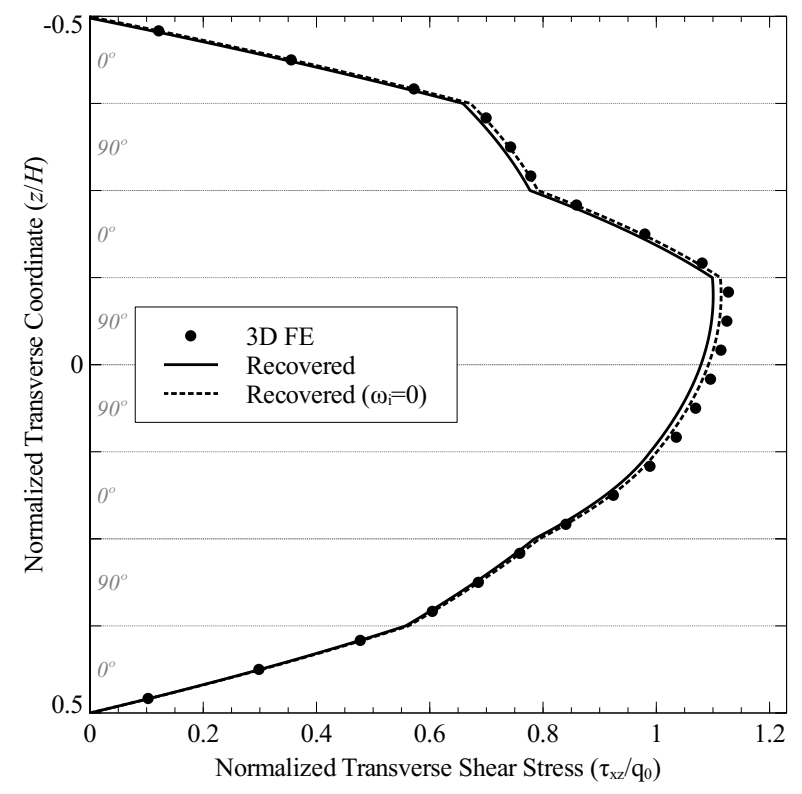

(a) Transverse shear stress $\tau_{x z}$.

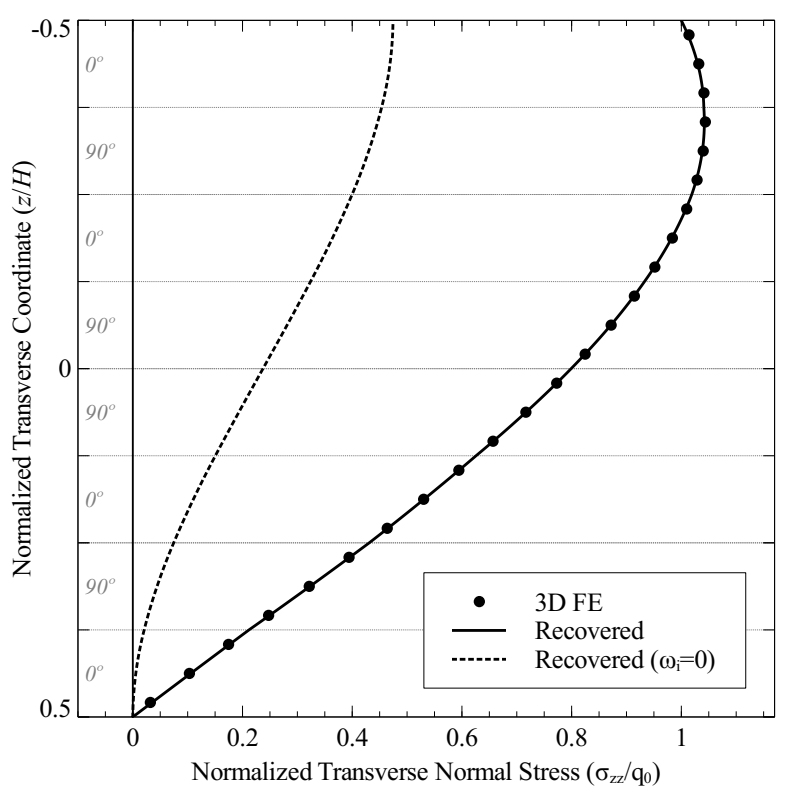

(c) Transverse normal stress $\sigma_{z z}$.

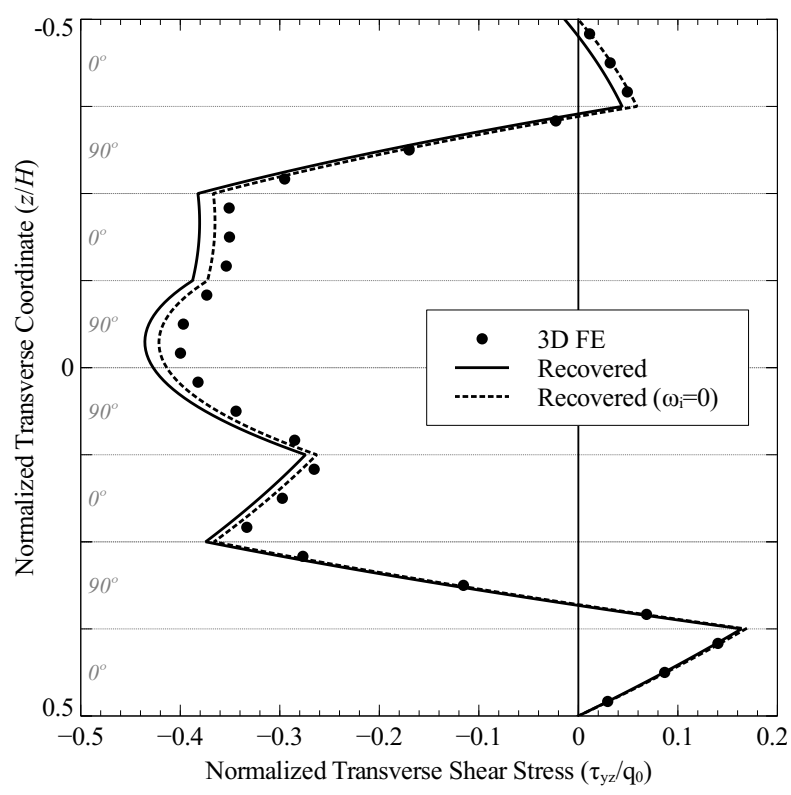

(b) Transverse shear stress $\tau_{y z}$.

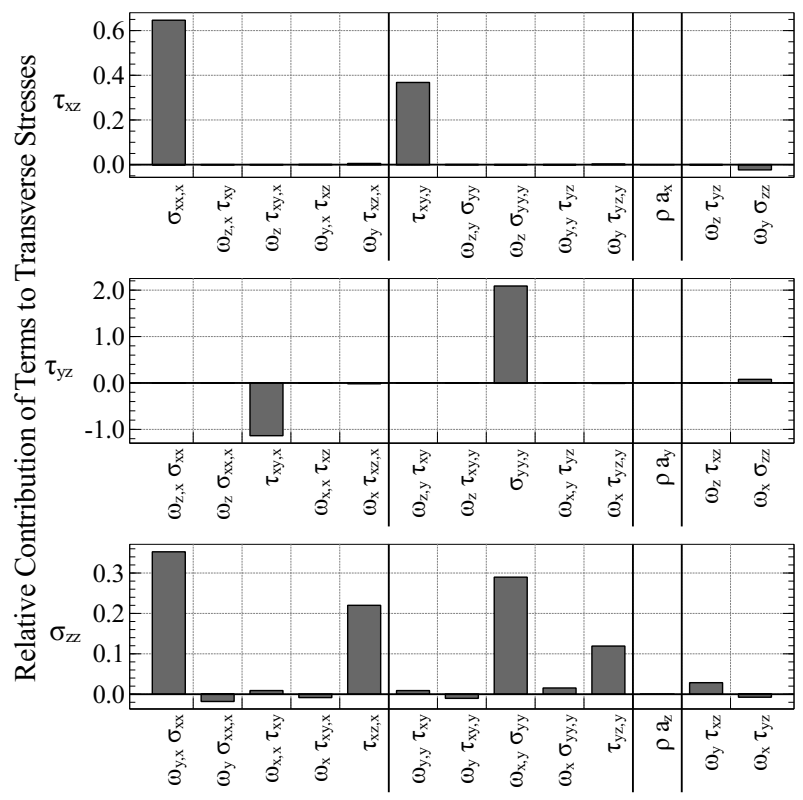

(d) Contributions of terms of Table 3.1.

Figure 5.10: Results of stress recovery at Point A, the quarter-point. 
again with the same sets of data as in Figures 5.10a and 5.10b. The distribution of the geometrically nonlinear recovery procedure shows excellent agreement with the benchmark solution, as well as with the known traction boundary condition at the top surface of the laminate. In this case, the results of neglecting the rotation terms in the stress recovery procedure result in a grossly misrepresented stress distribution, given in the dashed line of Figure 5.10c.

Finally, Figure 5.10d shows the relative contribution of each term of the integrands of Eq. (3.14) to the distribution of the three recovered transverse stresses. Each of the three plots corresponds to the three transverse stresses $\tau_{x z}, \tau_{y z}$, and $\sigma_{z z}$, and likewise, the three columns of Table 3.1. The first eleven columns of each plot correspond to the eleven rows of terms in Table 3.1-the eleven terms in the integrands of Eq. (3.14). (Note that in the current quasi-static analysis, the inertial terms, and the associated columns, are identically zero.) The remaining two columns are the contribution of the other two transverse stresses to the stress of interest (the effect of solving the matrix equation Eq. (3.11)).

The normalized contribution of each term is then computed as follows. Consider the first plot of Figure 5.10d, that for $\tau_{x z}$. The first column of that plot represents the term $\sigma_{x x, x}$ that appears in the integral equation for $\tau_{x z}$ (Eq. (3.4a)). If only that term $\left(\sigma_{x x, x}\right)$ were to be integrated to find $f_{x z}$ in Eq. (3.8), the resulting distribution of $f_{x z}$ would be that given in Figure 5.11a. Integrating that distribution, considering only the portion of the curve that is positive, gives a value of $0.151 \mathrm{Nm}$. Likewise, integrating considering only the portion of the curve that is negative gives $-146.4 \mathrm{~N} \mathrm{~m}$. The integral of the recovered $\tau_{x z}$ (Figure 5.11c) is $-226.2 \mathrm{Nm}$. Thus, $\sigma_{x x, x}$ contributes to the distribution of $\tau_{x z}$ towards a positive stress by

$$
\frac{\int_{H / 2}^{-H / 2}\left(+\int_{H / 2}^{z} \sigma_{x x, x} \mathrm{~d} z\right) \mathrm{d} z}{\int_{H / 2}^{-H / 2} \tau_{x z} \mathrm{~d} z}=\frac{0.151 \mathrm{Nm}}{-226.2 \mathrm{Nm}}=-6.68 \times 10^{-4}
$$




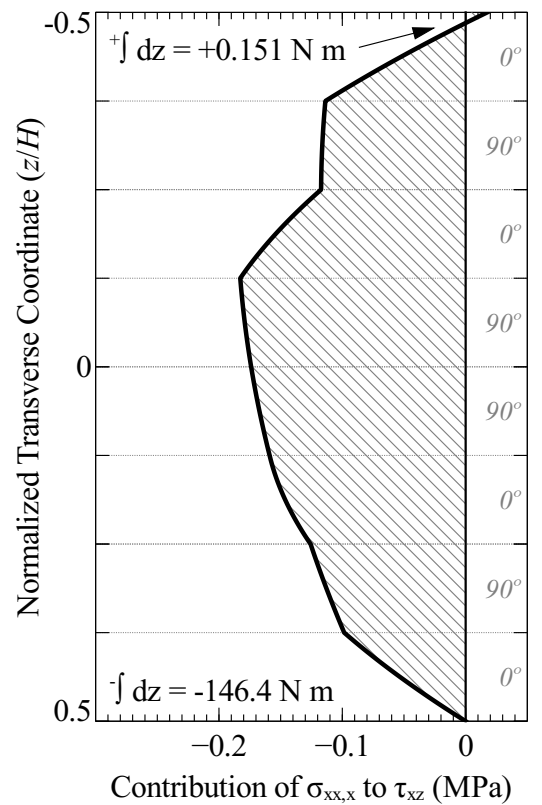

(a) Contribution from $\sigma_{x x, x}$.

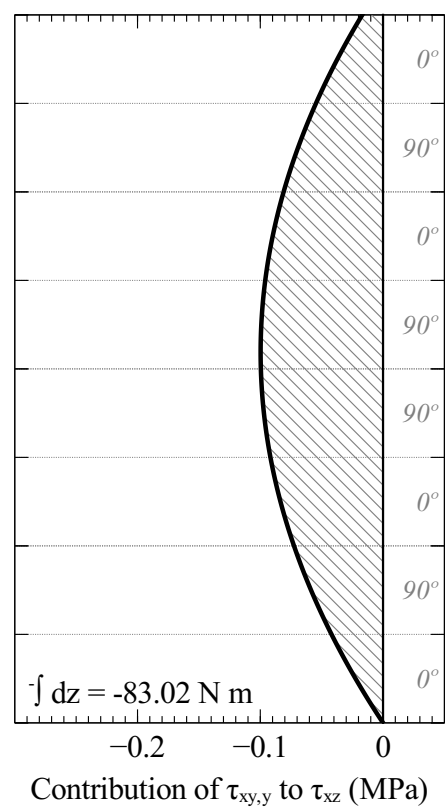

(b) Contribution from $\tau_{x y, y}$.

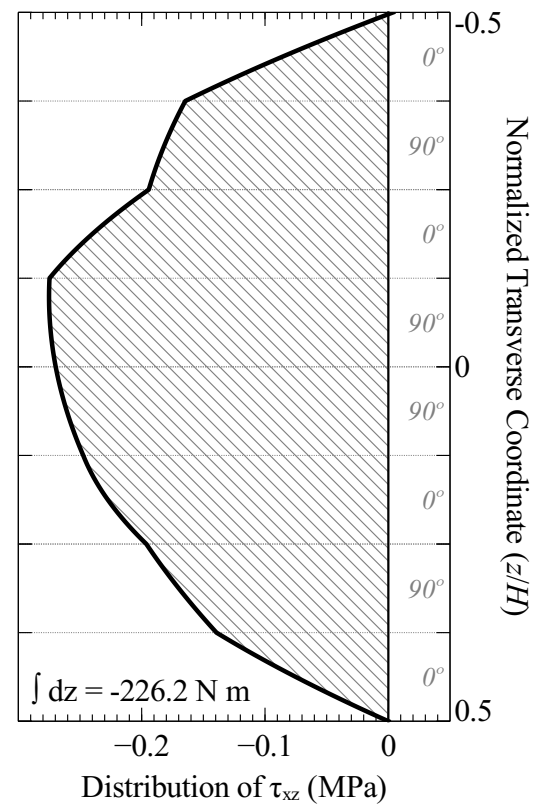

(c) Total distribution of $\tau_{x z}$.

Figure 5.11: Contribution of individual terms from Table 3.1 to $\tau_{x z}$ at quarter-point.

Likewise, $\sigma_{x x, x}$ contributes to the distribution of $\tau_{x z}$ towards a negative stress by

$$
\frac{\int_{H / 2}^{-H / 2}\left(-\int_{H / 2}^{z} \sigma_{x x, x} \mathrm{~d} z\right) \mathrm{d} z}{\int_{H / 2}^{-H / 2} \tau_{x z} \mathrm{~d} z}=\frac{-146.4 \mathrm{Nm}}{-226.2 \mathrm{~N} \mathrm{~m}}=0.647
$$

The positive contribution of $\sigma_{x x, x}$ is negligible, but $\sigma_{x x, x}$ contributes negatively $64.7 \%$ toward the total distribution of $\tau_{x z}$. The first column of the $\tau_{x z}$ plot of Figure 5.10d then shows a normalized contribution of 0.647 . (The bottom of the bar in the first column is in fact at $-6.68 \times 10^{-4}$, but cannot be distinguished because of the scale of the plot.)

Figure 5.10d may now be used to determine which terms (tabulated in Table 3.1) of the geometrically nonlinear equilibrium equations are necessary to consider in the recovery of the transverse stresses. Considering $\tau_{x z}$, the first and sixth columns, corresponding to $\sigma_{x x, x}$ and $\tau_{x y, y}$, are the key contributors, while the last column, $\omega_{y} \sigma_{z z}$, contributes a fraction in the negative direction. The last term is in fact the visible difference between the solid and dashed lines of Figure 5.10a. The contribution of the remaining terms is negligible. 
The terms which contribute to the recovery of $\tau_{y z}$ are those represented in the third, eighth, and thirteenth columns of the center plot in Figure 5.10d, $\tau_{x y, x}, \sigma_{y y, y}$, and $\omega_{x} \sigma_{z z}$. Again, the last term contributes to the visible difference between the solid and dashed lines of Figure 5.10b.

Finally, the bottom plot of Figure 5.10d depicts the contributions of terms to $\sigma_{z z}$. The key terms appear to be $\omega_{y, x} \sigma_{x x}, \tau_{x z, x}, \omega_{x, y} \sigma_{y y}$, and $\tau_{y z, y}$. However, if the third and sixth columns, $\omega_{x, x} \tau_{x y}$ and $\omega_{y, y} \tau_{x y}$, are added to this list, the remaining columns contribute a combined total of only $0.046 \%$ to the distribution of $\sigma_{z z}$. These six terms match those given in the equilibrium equations of Byun and Kapania [34] in Eq. (2.4).

\subsubsection{Recovered Stresses near Plate Center, Point B}

Our attention now turns toward the center of the plate, with the recovered stresses at Point $\mathrm{B}$ shown in Figure 5.12. The organization of the plots is identical to those of Point $\mathrm{A}$ in Figure 5.10.

Again, the geometrically linear stress recovery procedure shows better agreement with the known zero shear traction at the top surface compared to the geometrically nonlinear stress recovery. Although the transverse shear stress $\tau_{x z}$ appears to show a large discrepancy between the various solution methods, this stress is an order of magnitude less than the opposing transverse shear stress $\tau_{y z}$.

Considering the transverse normal stress distribution in Figure 5.12c, the geometrically nonlinear stress recovery procedure again shows excellent agreement with the benchmark solution, while the geometrically linear stress recovery procedure actually predicts a tensile traction at the top surface.

Finally, the key terms which contribute to the recovery of the transverse stresses near the center of the plate are within those given by Byun and Kapania [34] in Eq. (2.4). 


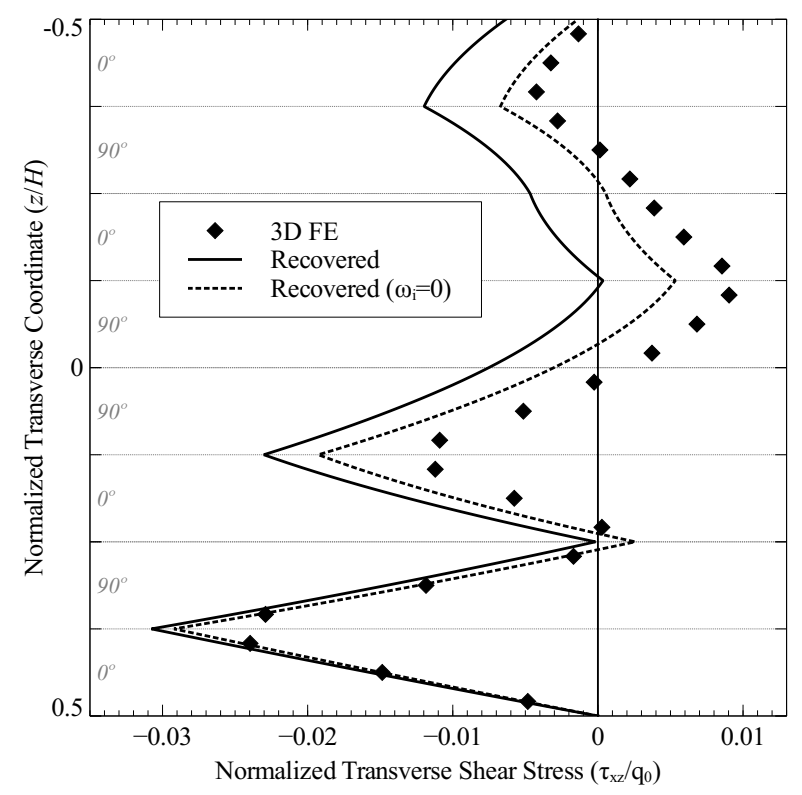

(a) Transverse shear stress $\tau_{x z}$.

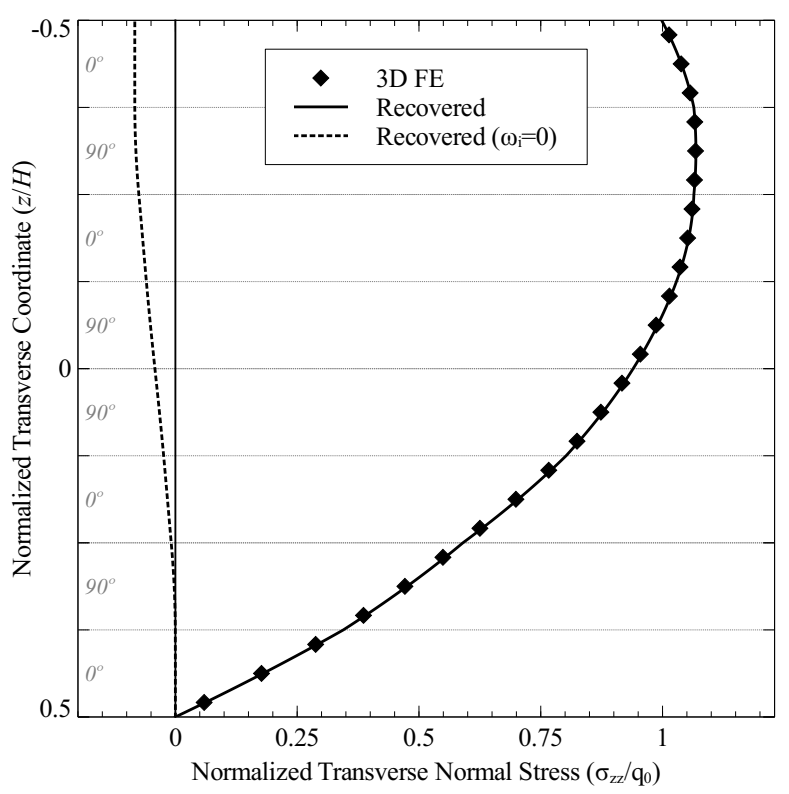

(c) Transverse normal stress $\sigma_{z z}$.

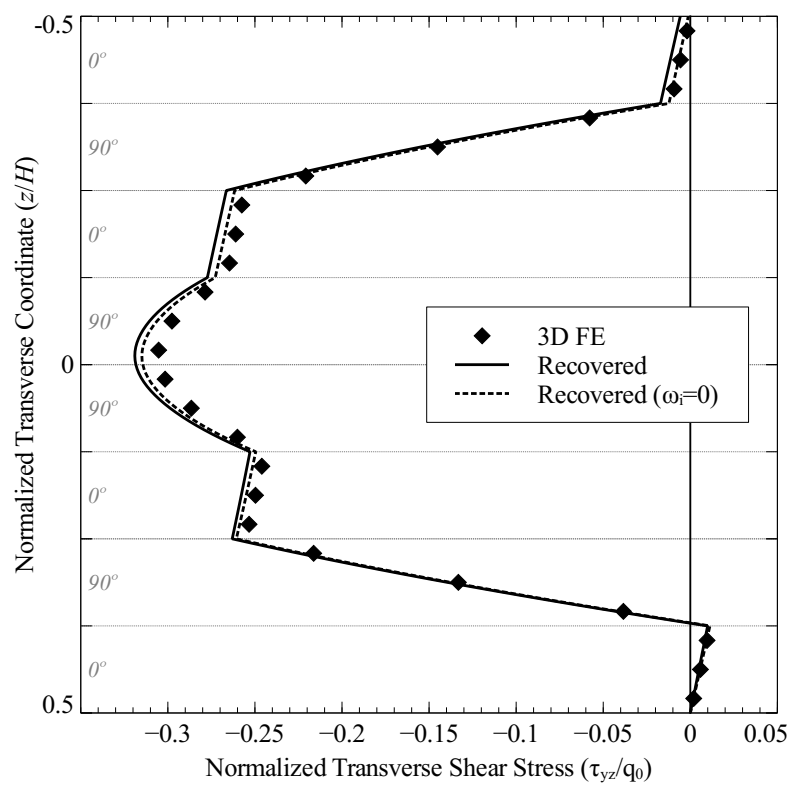

(b) Transverse shear stress $\tau_{y z}$.

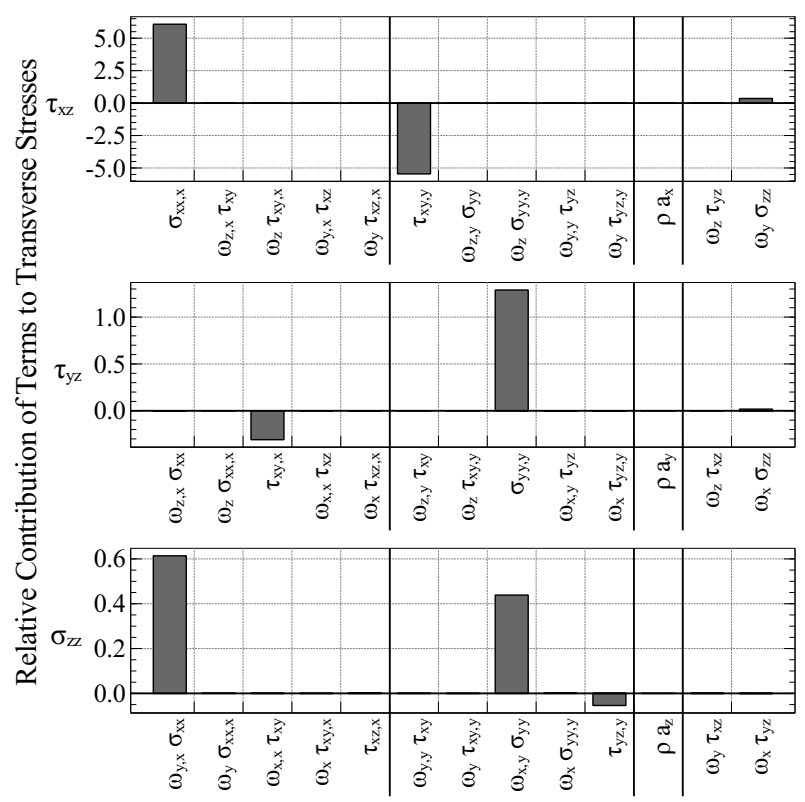

(d) Contributions of terms of Table 3.1.

Figure 5.12: Results of stress recovery at Point B, near plate center. 


\subsubsection{Recovered Stresses near $x^{+}$-Edge, Point C}

With Figure 5.13, attention now turns to the recovered stresses near the center of the supported $x=a / 2$ edge, Point $\mathrm{C}$, where the shear stress $\tau_{x z}$ is expected to be at its peak magnitude.

The peak magnitude of $\tau_{x z}$ in Figure 5.13a is nearly 13 times that of the applied normal traction at the top surface. The distributions of $\tau_{x z}$ from the geometrically linear and nonlinear stress recovery procedures are nearly identical, and show excellent agreement with the benchmark solution.

This distribution of $\tau_{y z}$ in Figure 5.13b appears better with the contribution of the nonlinear term $\omega_{x, x} \tau_{x z}$ (see Figure 5.13d; neglected by Byun and Kapania [34]). Additionally, in this case the geometrically nonlinear recovery better predicts the top surface traction $\left(\tau_{y z}=0\right)$ and overall distribution compared to the geometrically linear recovery method $\left(\omega_{x}=\omega_{y}=\omega_{z}=0\right)$. However, both methods underestimate the shear stress compared to the benchmark solution.

The recovered transverse normal stress from the geometrically nonlinear procedure closely predicts the known traction at the top surface (although it is over-predicted by $0.15 \%$ ). Again, the benchmark solution shows some lack of convergence in the $0^{\circ}$-layers, as the known traction boundary conditions do not appear satisfied.

Although some terms absent from Byun and Kapania [34] appear to contribute to the recovery of $\sigma_{z z}$, their combined contribution is $0.29 \%$.

\subsubsection{Recovered Stresses near $y^{+}$-Edge, Point D}

Lastly, with Figure 5.14, consider the recovered stresses near the center of the supported $y=b / 2$ edge, where the shear stress $\tau_{y z}$ is expected to be at its peak magnitude. In this case, the peak magnitude of the shear stress $\tau_{y z}$ is nearly 12 times that of the applied normal traction at the top surface.

The results are similar to those at Point $\mathrm{C}$ given (Figure 5.13). The distribution of the 


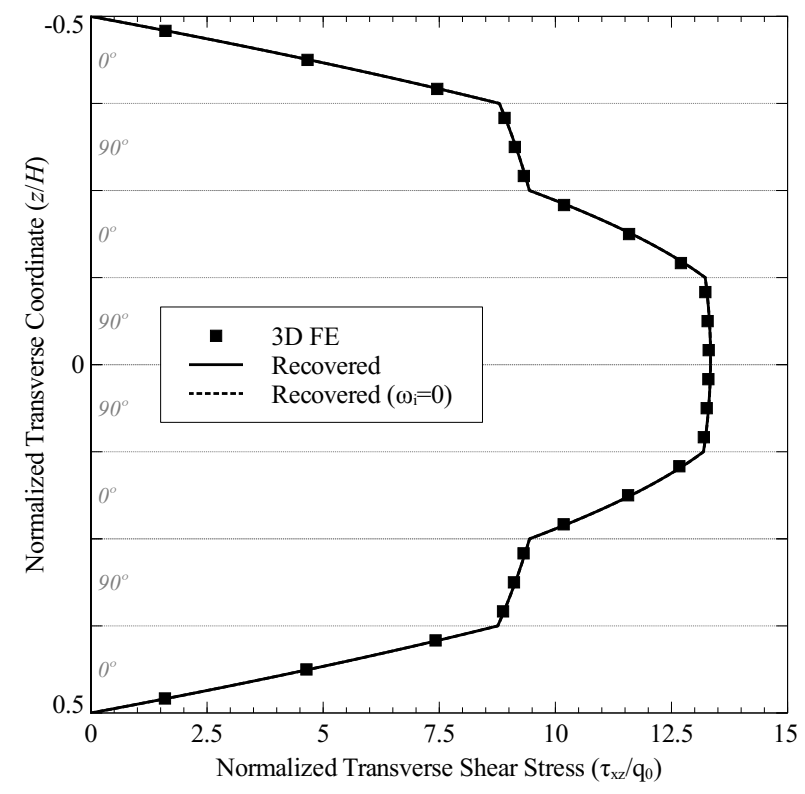

(a) Transverse shear stress $\tau_{x z}$.

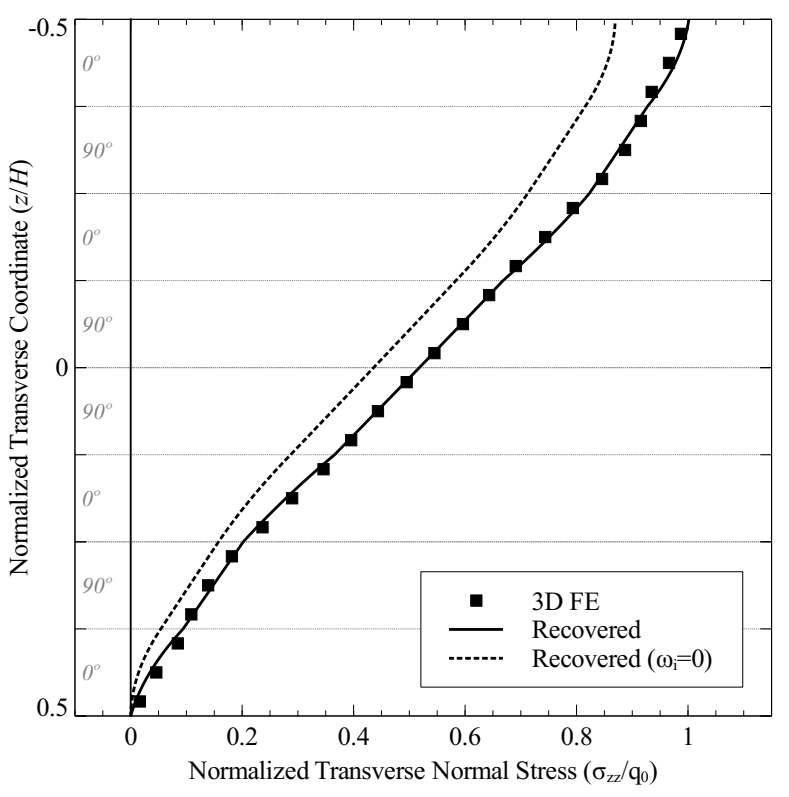

(c) Transverse normal stress $\sigma_{z z}$.

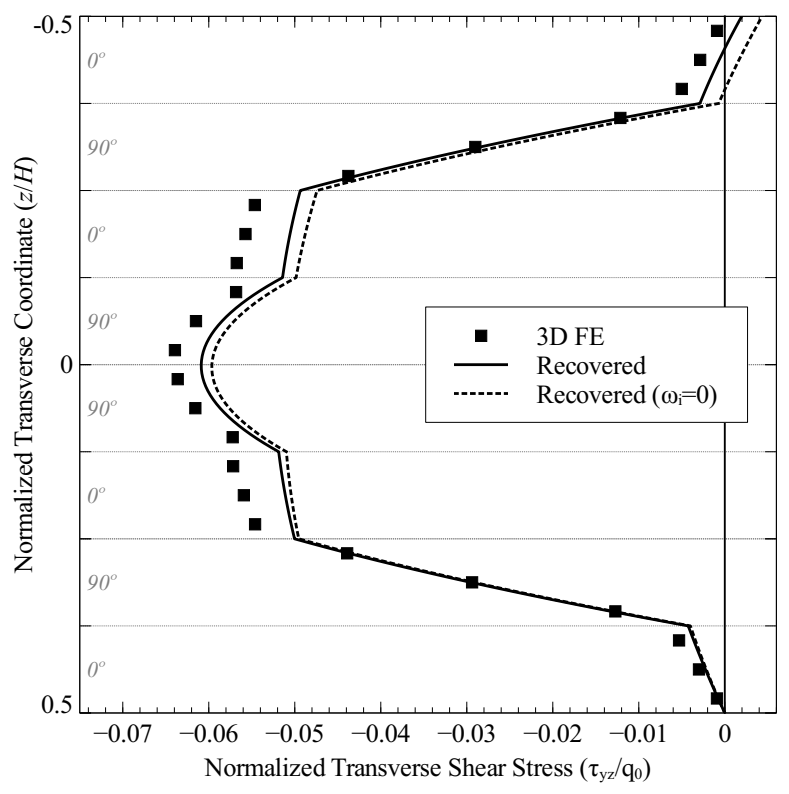

(b) Transverse shear stress $\tau_{y z}$.

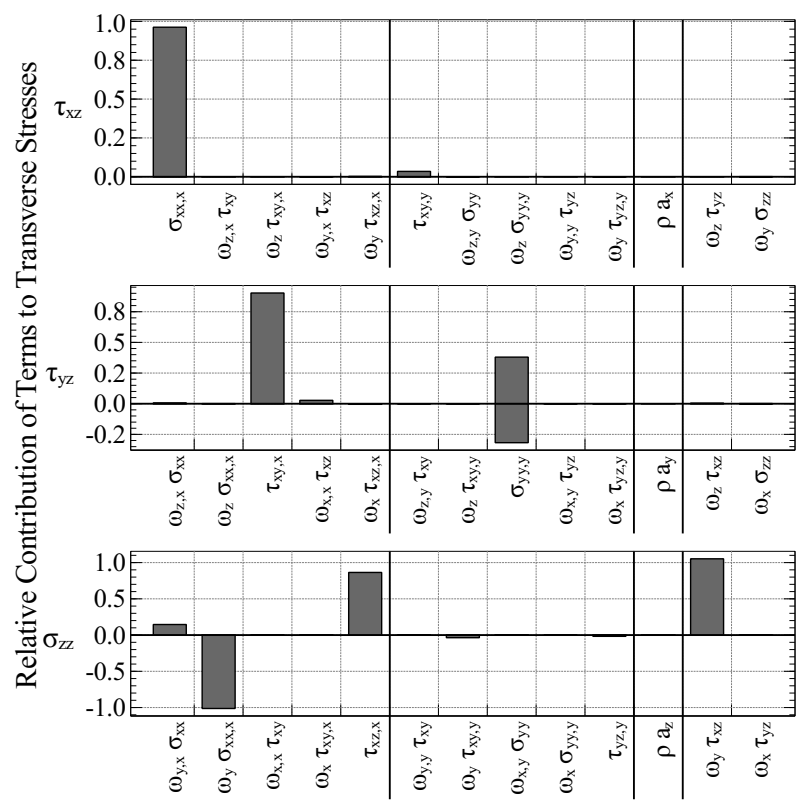

(d) Contributions of terms of Table 3.1.

Figure 5.13: Results of stress recovery at Point C, near $x^{+}$-edge. 


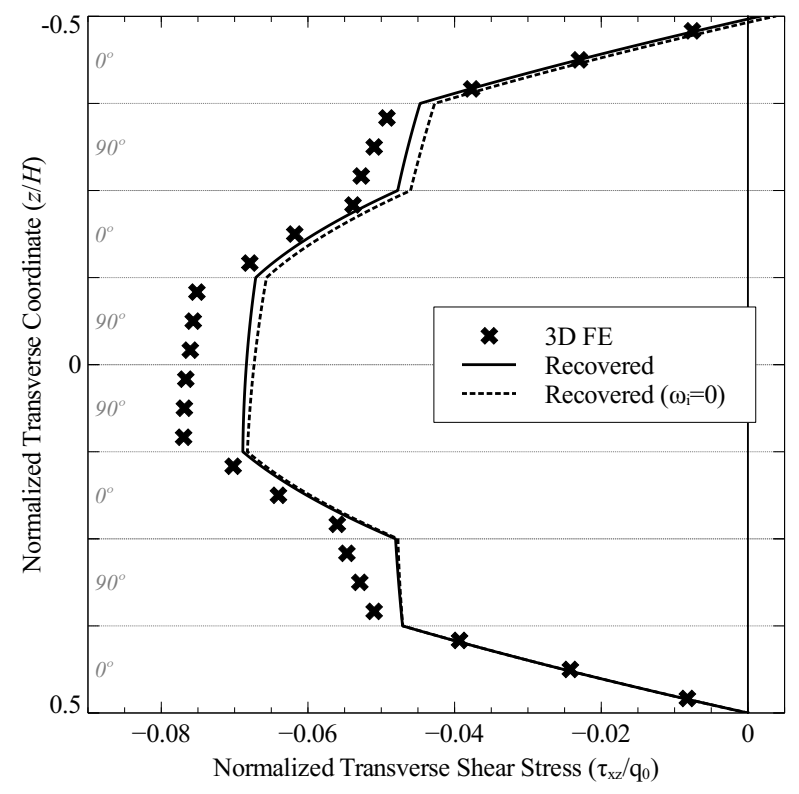

(a) Transverse shear stress $\tau_{x z}$.

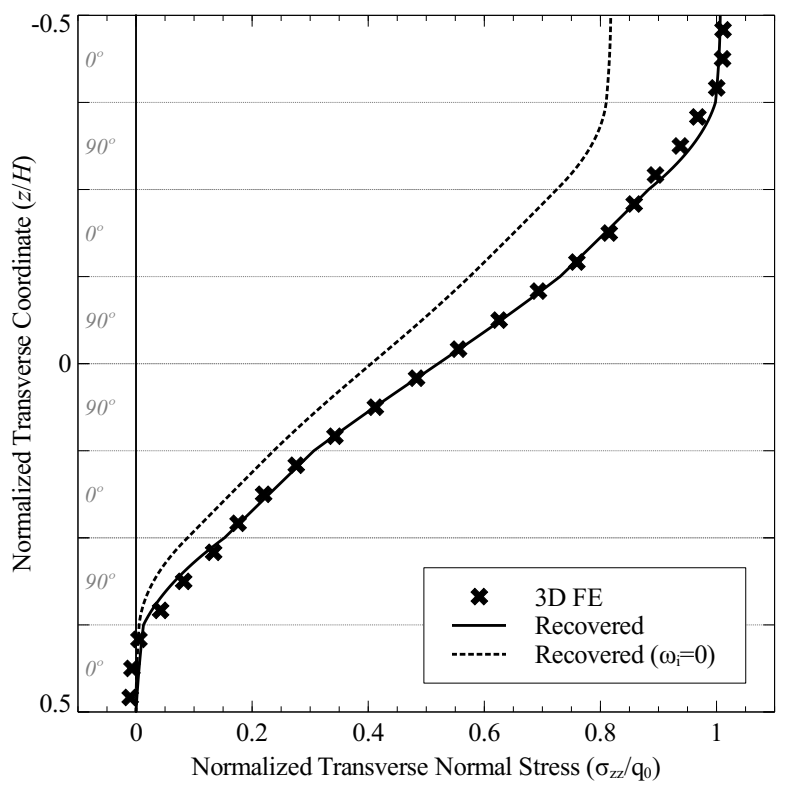

(c) Transverse normal stress $\sigma_{z z}$.

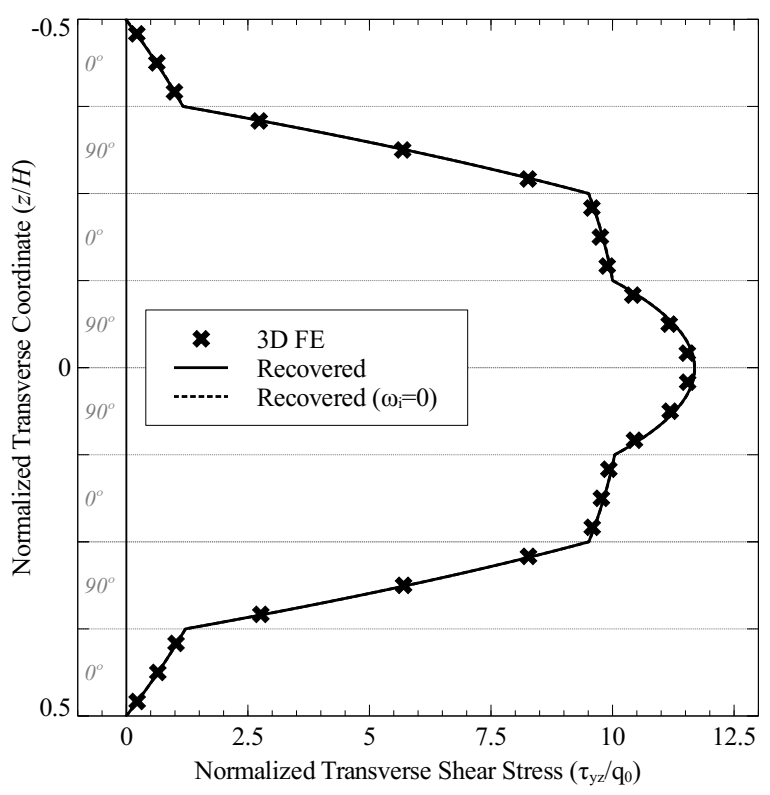

(b) Transverse shear stress $\tau_{y z}$.

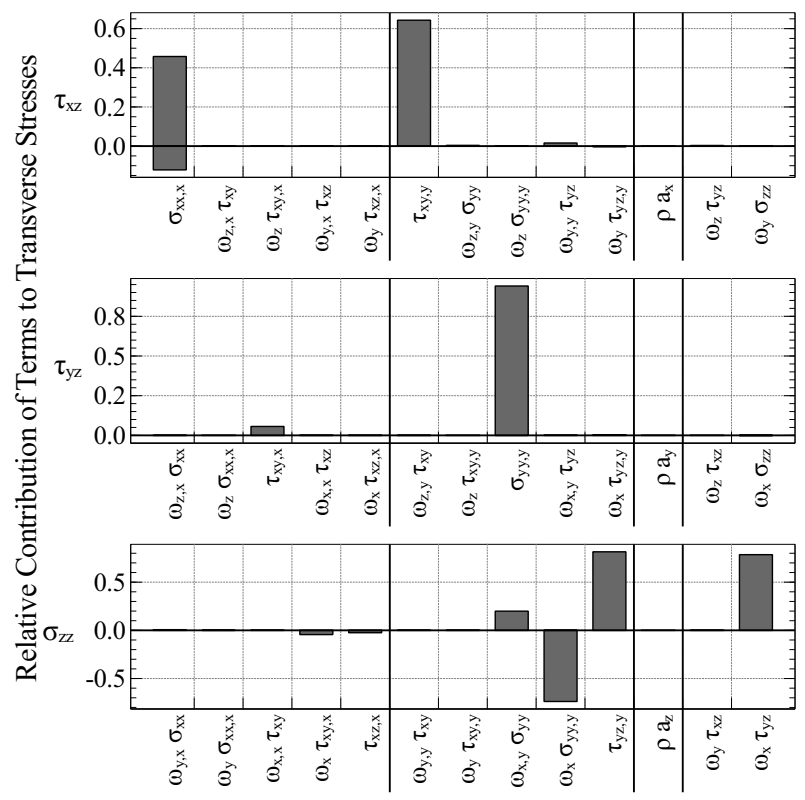

(d) Contributions of terms of Table 3.1.

Figure 5.14: Results of stress recovery at Point D, near ${ }^{+} y$-edge. 
recovered shear stress $\tau_{y z}$, both from the geometrically linear and nonlinear stress recovery procedures, shows good agreement with that from the benchmark solution. Both procedures underestimate the second shear stress $\tau_{x z}$, although the recovered stress from the geometrically nonlinear procedure appears slightly better.

The transverse normal stress recovered from the geometrically nonlinear procedure again closely predicts the top surface boundary condition (the value is $0.64 \%$ higher than the applied traction), while the recovery neglecting nonlinear terms is removed from the true distribution. Using only terms from Byun and Kapania [34] improves the predicted top surface boundary condition by $0.31 \%$.

\subsubsection{Analysis of Convergence of Recovery Procedure}

Two aspects of convergence of the geometrically nonlinear stress recovery procedure must be discussed. The procedure depends on the convergence of the solution of a plate-type finite element analysis, not only in the displacements and stresses, but also in the derivatives of these. In the recovery of the transverse normal stress, second derivatives of these quantities are important. Secondly, the iteration process (Section 3.2) of the stress recovery procedure must converge. These two types of convergence are discussed below.

\subsubsection{Convergence of Finite Element Results}

Figure 5.15 shows the three recovered transverse stresses at the quarter-point (see Figure 5.10) for varying mesh densities of the two-dimensional finite element analysis, where the number in the legend indicates the number of elements along a side of the one-quarter plate model. The two transverse shear stresses are converged at a mesh of 35-by-35 elements (results are within 1\% of those given by the 95-by-95 mesh), although even a 15-by-15 mesh shows good results. (Recall from Section 5.2.1 that the in-plane response was converged at a 5-by-5 mesh.) The transverse normal stress is converged (again, results are within $1 \%$ of those given by more refined meshes) even with the 15-by-15 element mesh. Surprisingly, a 


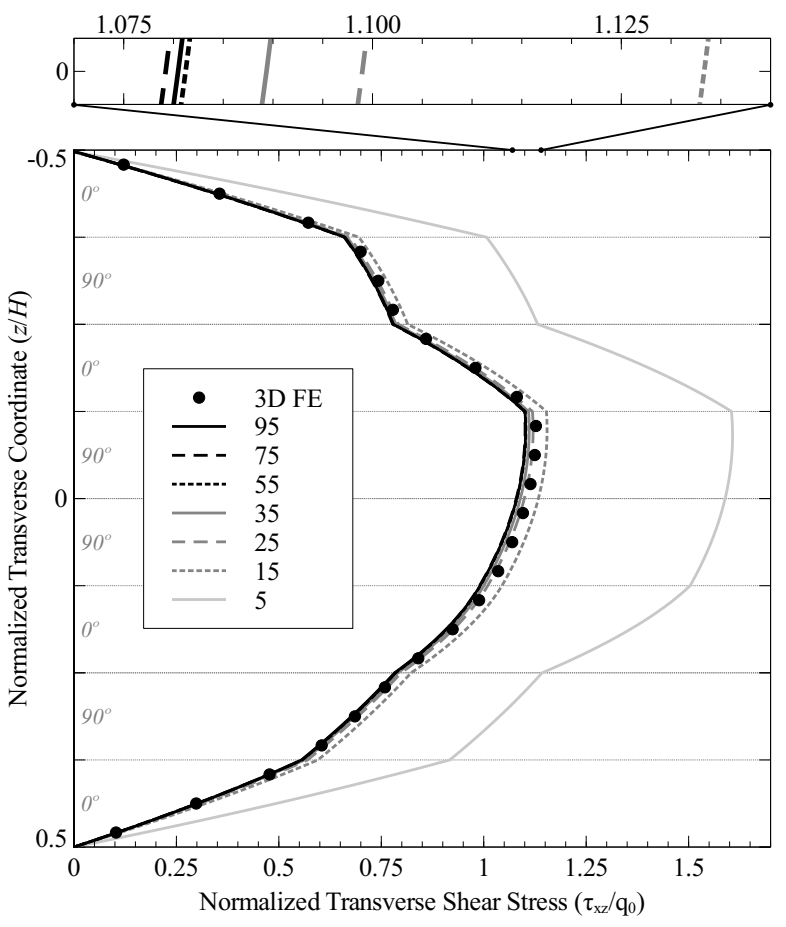

(a) Transverse shear stress $\tau_{x z}$.

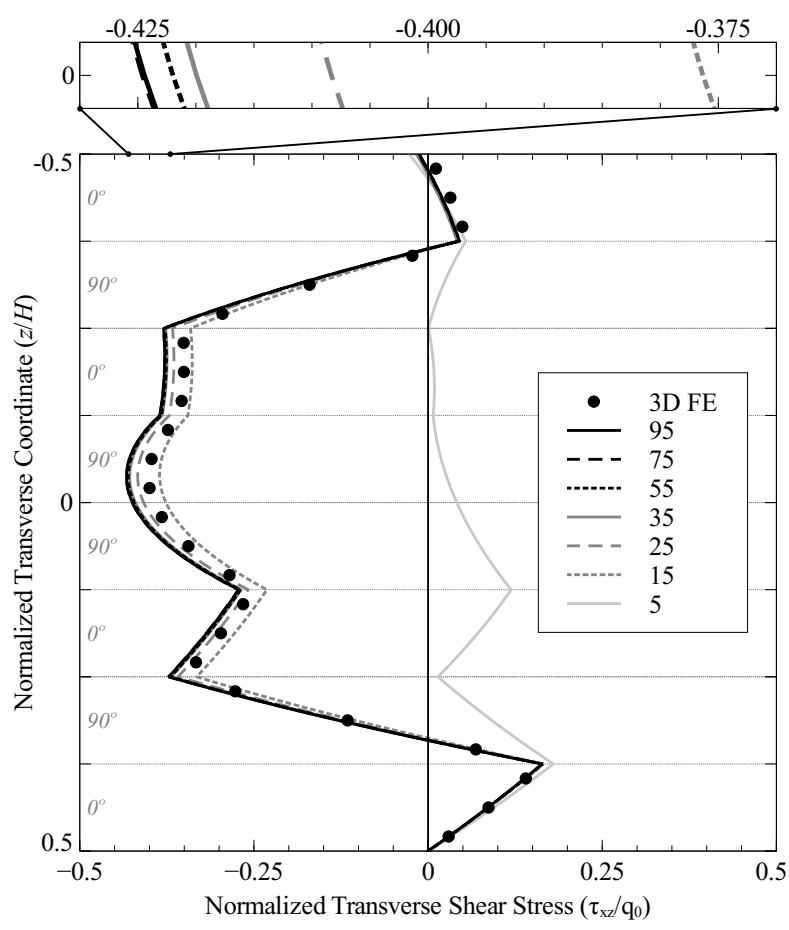

(b) Transverse shear stress $\tau_{y z}$.

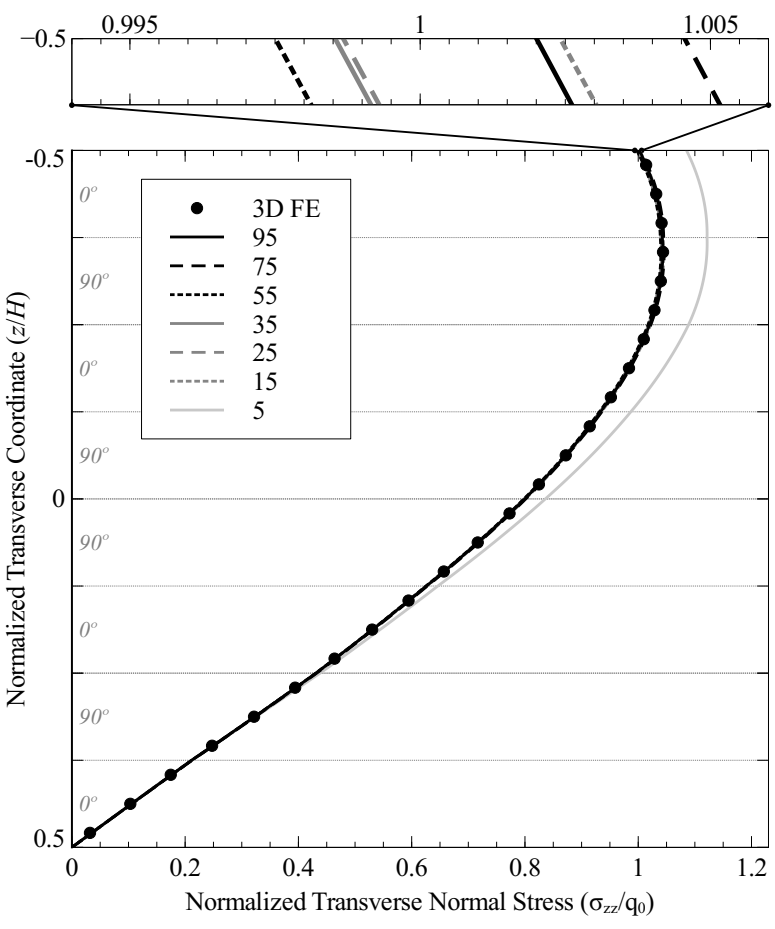

(c) Transverse normal stress $\sigma_{z z}$.

Figure 5.15: Convergence of finite element results at Point A, the quarter-point. 
very coarse five-by-five element mesh yields a transverse normal stress within $10 \%$ of the converged distribution. For two iterations of the stress recovery procedure, this five-by-five element mesh provides the minimum number elements required for current implementation of the stress recovery at the quarter-point.

\subsubsection{Convergence of the Iteration Procedure}

Figure 5.16 shows the three recovered transverse stresses at the quarter-point (see Figure 5.10) for successive iterations of the stress recovery procedure.

As shown by Figures 5.16a and 5.16b, a single iteration is sufficient for recovery of the transverse shear stresses. Because the transverse shear stresses depend primarily on the intralaminar stresses (see Figures 5.10d to 5.14d), which are unchanged from iteration to iteration, the iterating process does not add to the accuracy of the recovery of these stresses.

For the transverse normal stress, two iterations are required for a converged solution. The first iteration is accurate in its prediction of the traction boundary condition at the top surface, but lacks accuracy in the distribution of the transverse normal stress. The shear-deformable finite element solution provides accurate (in an integral sense) values of the transverse shear stresses, which allows for the quasi-accurate recovery of the first iteration. However, for the true distribution to be recovered, the distribution of the transverse shear stresses (specifically, their derivatives) must be accurately known. These accurate distributions are not available until after the first iteration of the procedure.

Were a Kirchhoff-type element formulation to be used rather than the shear-deformable formulation, the conclusions of this section would remain the same, although the first recovery iteration of the transverse normal stress would yield an inaccurate prediction of the top surface traction boundary condition, in addition to an inaccurate distribution through the thickness. 


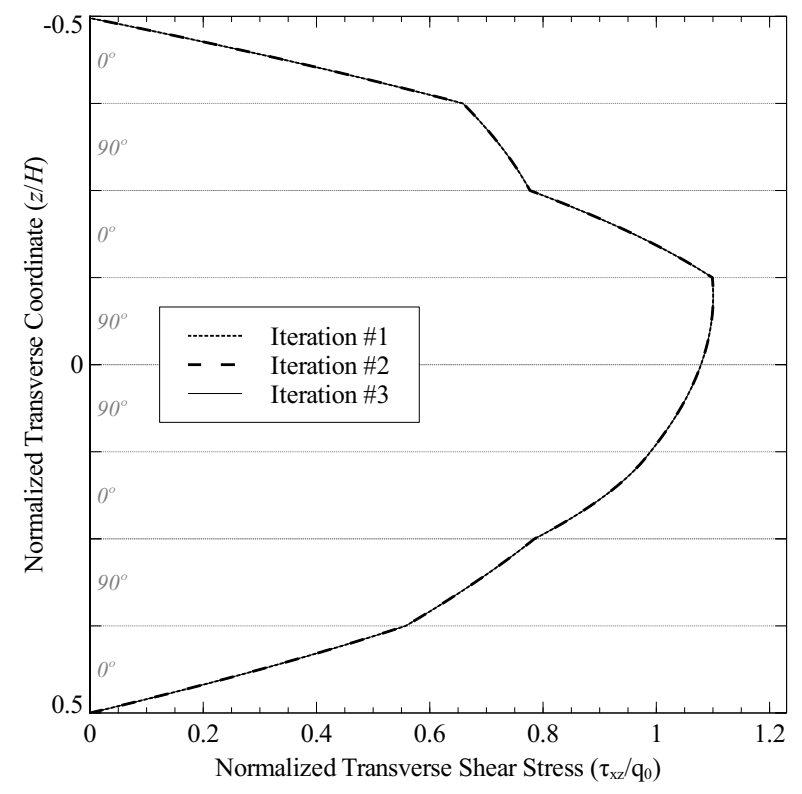

(a) Transverse shear stress $\tau_{x z}$.

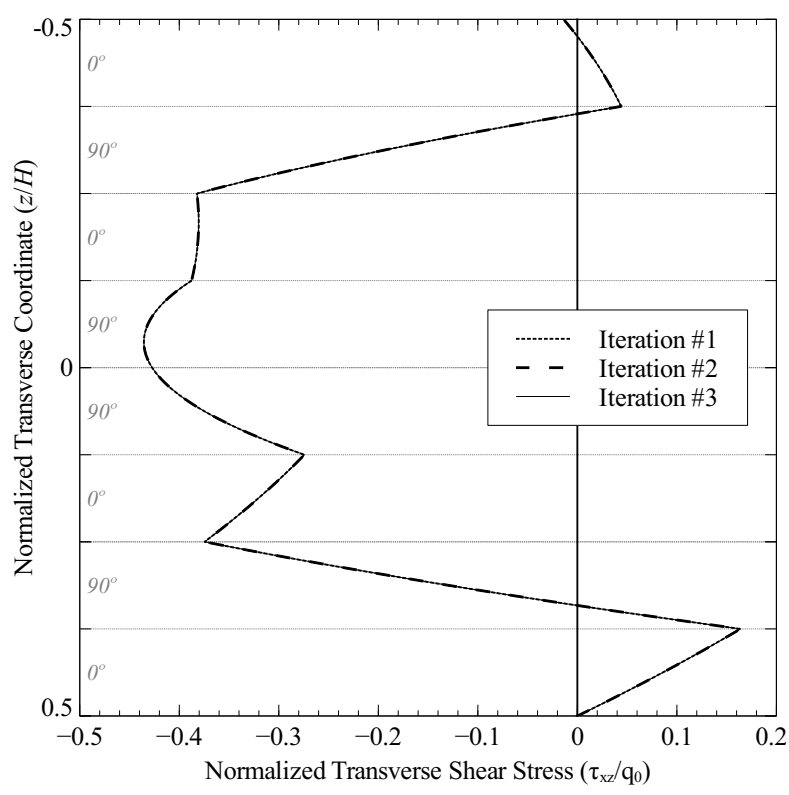

(b) Transverse shear stress $\tau_{y z}$.

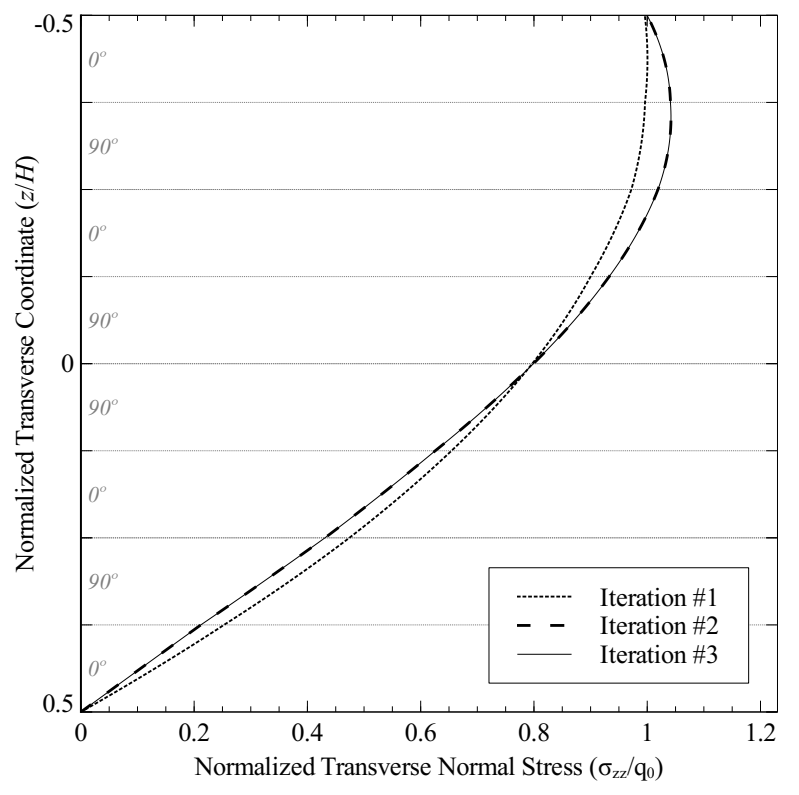

(c) Transverse normal stress $\sigma_{z z}$.

Figure 5.16: Convergence of iteration procedure at Point A, the quarter-point. 


\subsubsection{Analysis of Accuracy of Recovery Procedure}

While the accuracy of the geometrically nonlinear stress recovery procedure has been demonstrated for a single example problem, a knowledge of the limits of the process is important. Bounds for the applicability of the geometrically nonlinear stress recovery procedure are now sought. Four criteria are considered:

- relative thickness of the plate (span-ratio $a / H)$

- magnitude of rotations of plate elements

- location within the plate

- types of loading distributions

The relative thickness of the plate does not directly affect the stress recovery procedure, but rather affects the accuracy of the intralaminar stresses as calculated by a plate-type analysis. The magnitude of rotations of plate elements must be considered, as the equilibrium equations are based on the assumption that the rotations are moderate. Further, certain locations in a finite element analysis are known to present difficulties in determining the mechanical response of a body, particularly near boundaries. Finally, the accuracy of the recovery procedure in analyzing a non-uniformly loaded plate will be determined.

\subsubsection{Accuracy with Varying Plate Span-Ratio}

For the recovered stresses of Figures 5.10 and 5.12 to 5.14 shown previously, a moderately thick plate with a span-ratio $a / H=60$ was considered. This span-ratio is now varied to consider thicker and thinner plates. A two-dimensional solution is known to lose accuracy with increasing plate thickness (span-ratio). Likewise, higher-order plate theories are known to have difficulties resolving stresses for thinner (higher span-ratio) plates. Figure 5.17a shows the percent error between the recovered transverse normal stress at the top surface of 
the laminate compared to the known traction boundary condition at the top surface, as a function of plate span-ratio.

In order to compare similar quantities as the plate span-ratio is varied, the ratio of the plate span to the deflection at the center of the plate was kept close to constant. That is, for the results shown in Figure 5.17, the quantity $a / w$ remains relatively constant at a value of 60 . For the previously considered plate with a span-ratio $a / H=60$, this corresponds to a center plate deflection of one plate thickness. The variation of $a / w$ with the remaining span-ratios considered is shown in Table 5.2.

As observed in Figure 5.17a, even for relatively thick plates, the error in the top surface traction at the four points considered is always below 3.5\%. While the thickest plate with $a / H=15$ deflects only a quarter of a plate thickness, the rotation of the geometrically nonlinear equilibrium equations contribute to the accuracy of the recovered stresses. As expected, thinner plates present less error in the recovery solution. Furthermore, the recovery procedure for extremely thin plates, even at a normalized center deflection of $w / H \approx 14$, gives

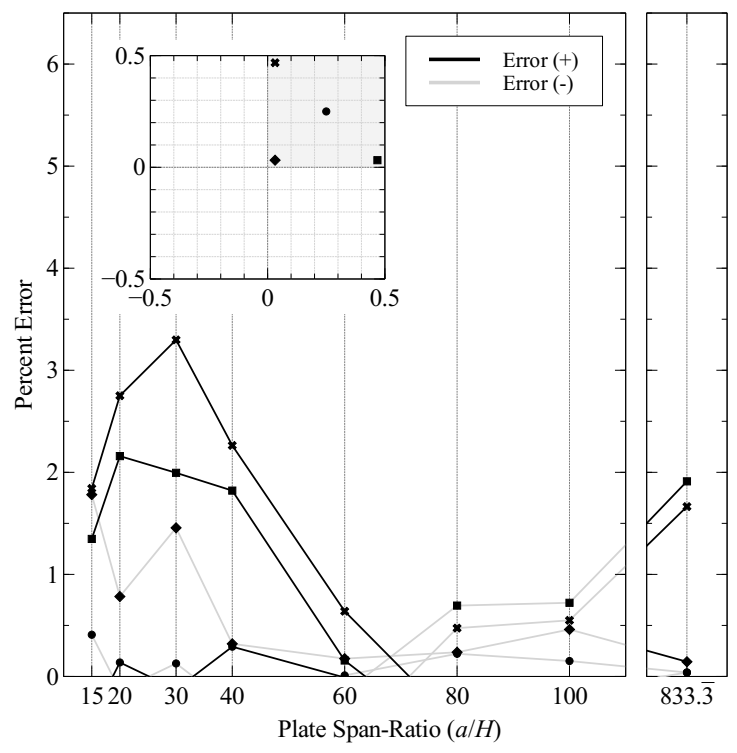

(a) Error.

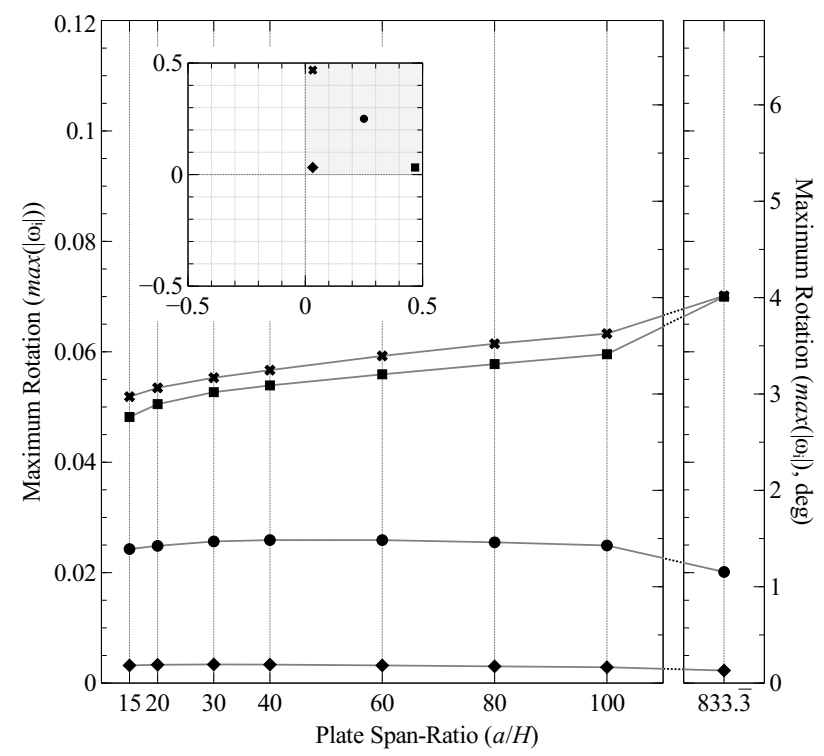

(b) Rotations.

Figure 5.17: Error in recovered $\sigma_{z z}$ at top surface and plate rotations as a function of plate span-ratio. 
Table 5.2: Details of solutions with varying span-ratios.

\begin{tabular}{ccccc}
\hline $\begin{array}{c}\text { Span Ratio } \\
(a / H)\end{array}$ & $\begin{array}{c}\text { Width } \\
(a, \mathrm{~mm})\end{array}$ & $\begin{array}{c}\text { Normalized Center } \\
\text { Deflection }(w / H)\end{array}$ & $\begin{array}{c}\text { Difference } \\
\text { from } a / w=60\end{array}$ & $\begin{array}{c}\text { Applied Traction } \\
(\mathrm{kPa})\end{array}$ \\
\hline 15 & 18 & 0.2502 & $-0.0766 \%$ & -5010 \\
20 & 24 & 0.3334 & $-0.0150 \%$ & -2400 \\
30 & 36 & 0.5002 & $-0.0317 \%$ & -914 \\
40 & 48 & 0.6667 & $-0.0050 \%$ & -501 \\
60 & 72 & 1.0001 & $-0.0083 \%$ & -250 \\
80 & 96 & 1.3326 & $0.0563 \%$ & -169 \\
100 & 120 & 1.6671 & $-0.0250 \%$ & -131 \\
$833 . \overline{3}$ & 1000 & 13.881 & $0.0580 \%$ & -16.33 \\
\hline
\end{tabular}

results with less than two percent error. Although the center deflection of the thickest plate $(a / H=15)$ is only one-quarter the plate thickness, accounting for geometric nonlinearities is still necessary in the stress recovery procedure, as demonstrated in the distribution of the transverse stresses shown in Figure 5.18.

While studying the effect of plate span-ratio on recovery convergence, an attempt was made to keep the plate rotations at the four locations (A, B, C, and D) the same for all span-ratios. The rotations at the four points for the various span-ratios are illustrated in Figure 5.17b and, as seen, the rotations did not vary much with span-ratio.

\subsubsection{Accuracy with Varying Plate Rotations}

Attention turns to how increasing plate rotations affect the recovery procedure. With a spanratio $a / H=60$, the error as a function of plate deflection, and thus plate rotations, in the recovered top surface boundary condition is shown in Figure 5.19a. Figure 5.19b shows the corresponding absolute value of the maximum of the three rotations $\left(\max \left(\left|\omega_{x}\right|,\left|\omega_{y}\right|,\left|\omega_{z}\right|\right)\right.$, or simply $\max |\omega|)$ for the four plate locations considered. Even for rotations of rather large magnitude, $\max |\omega| \approx 0.20\left(11.5^{\circ}\right)$, the error remains less than $7 \%$. Error in recovered stresses at points in the plate with less rotations (the quarter-point and near the center) remains low even for large relative plate deflections. 


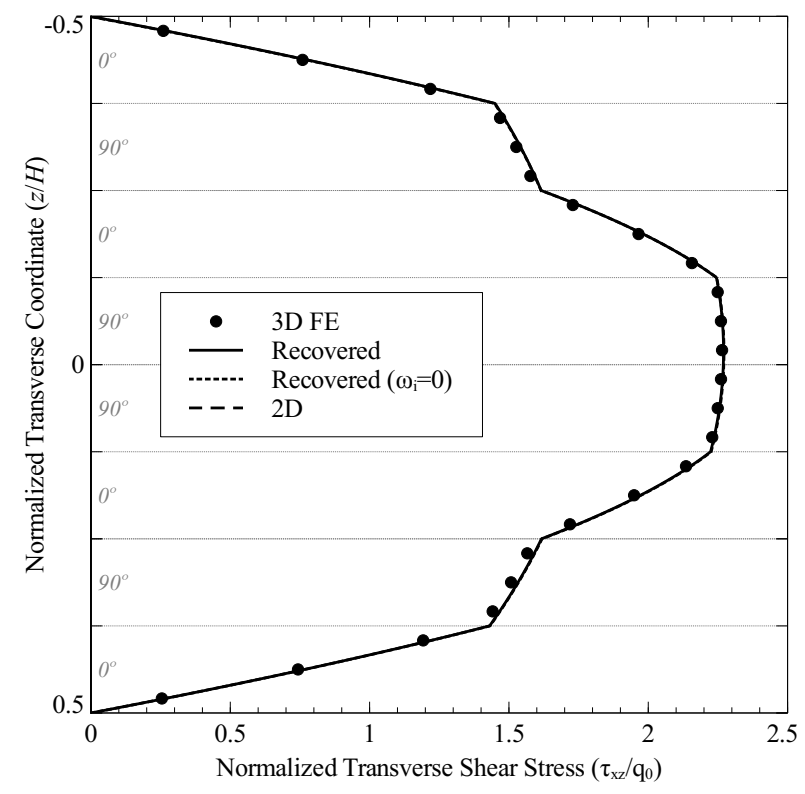

(a) Transverse shear stress $\tau_{x z}$.

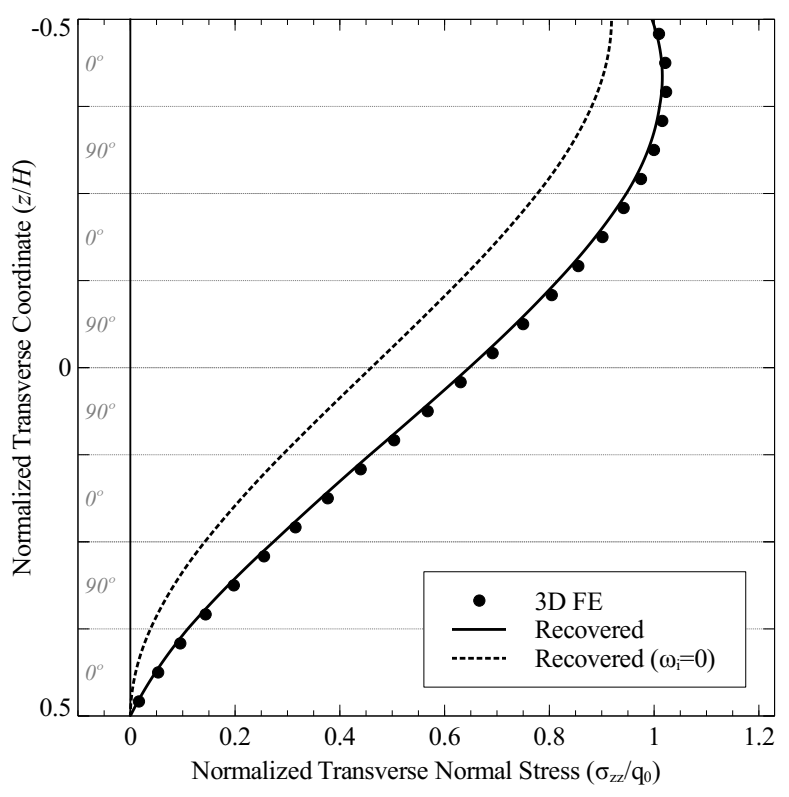

(c) Transverse normal stress $\sigma_{z z}$.

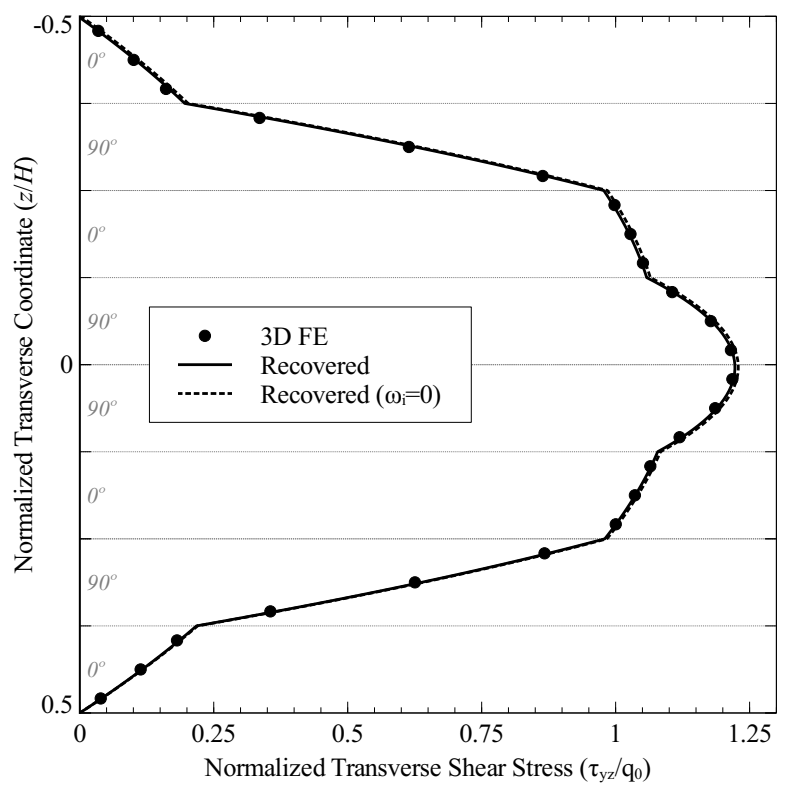

(b) Transverse shear stress $\tau_{y z}$.

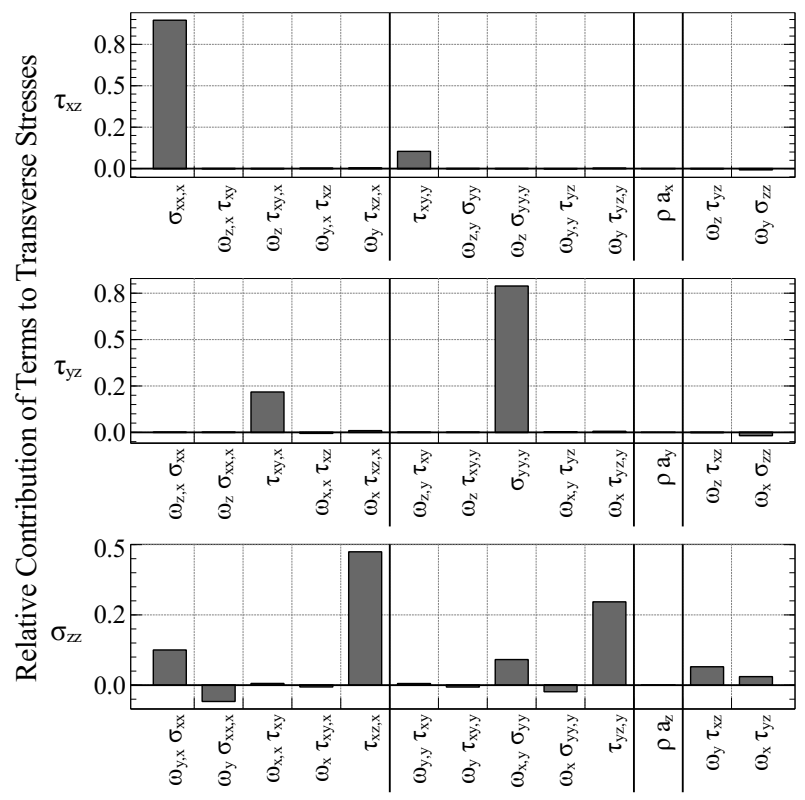

(d) Contributions of terms of Table 3.1.

Figure 5.18: Results of stress recovery at quarter-point of thick $(a / H=15)$ plate. 


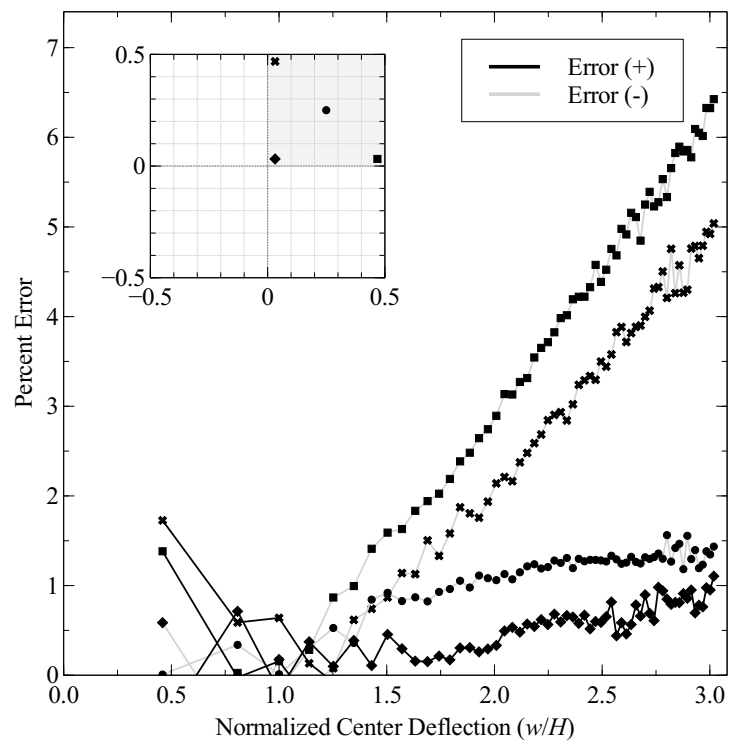

(a) Error.

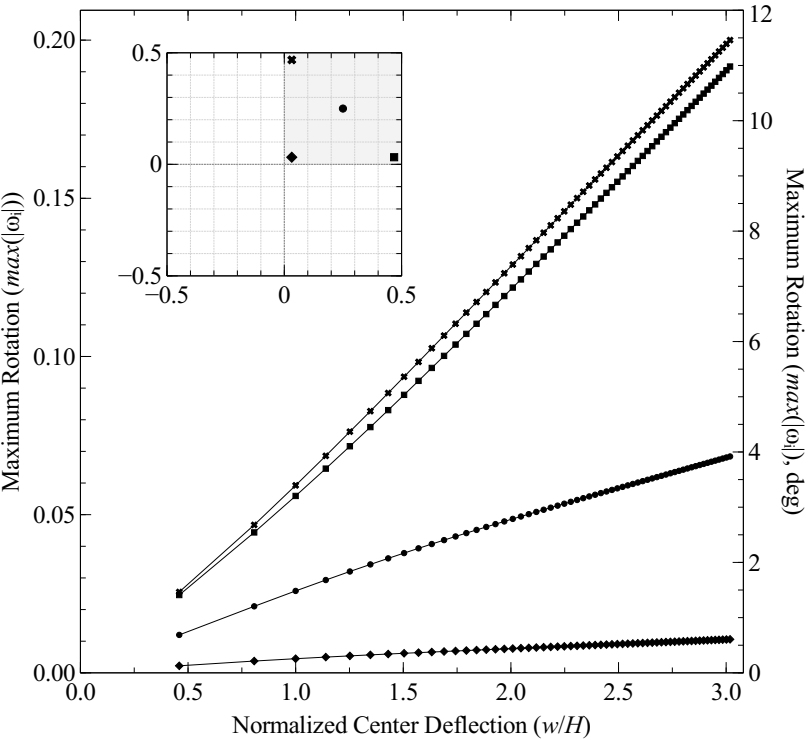

(b) Rotations.

Figure 5.19: Error in recovered $\sigma_{z z}$ at top surface and rotations as a function of plate deflection.

\subsubsection{Accuracy with Varying Locations within Plate}

To this point, only four discrete points have been considered for the recovery of the transverse stresses. The same plate with span-ratio $a / H=60$ and center plate deflection of $w / H=1$ is now used to determine the accuracy of the recovery procedure over the entire domain. Using a finite element mesh with 25 elements per edge of the quarter-model, the error in the predicted top surface boundary condition in $\sigma_{z z}$ compared to the known traction boundary condition is represented in Figure 5.20a. The plot covers the domain of the quarter-model plate, and is discretized to a 25 -by-25 mesh so that each square represents a single finite element. Because the current implementation of the stress recovery procedure requires plate response information from neighboring elements, elements on the boundary of the domain are omitted from the recovered solution and depicted with a filled pattern. The remaining elements in the domain are represented by a gray-scale fill - a white fill corresponds to a deviation of $0 \%$ from the known traction boundary condition, while a dark gray fill corresponds to a 


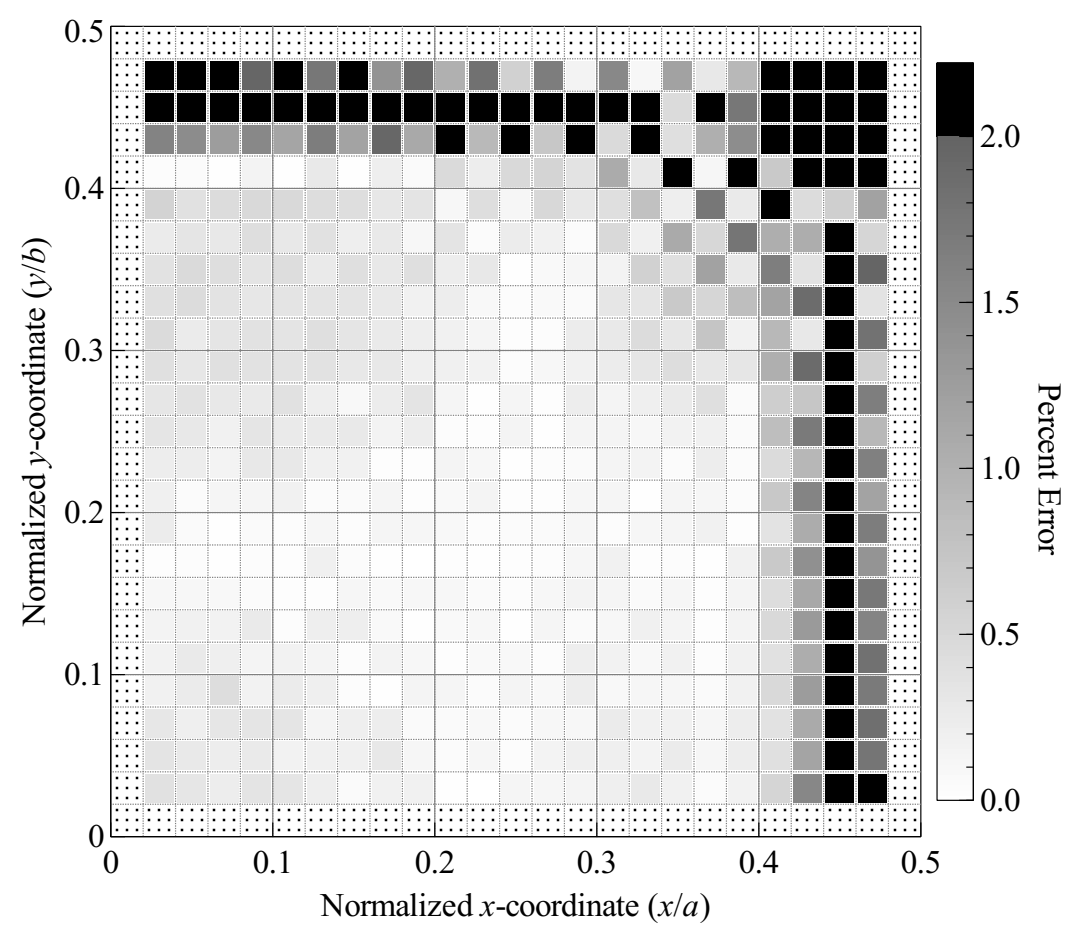

(a) Error for $25 \times 25$ mesh.

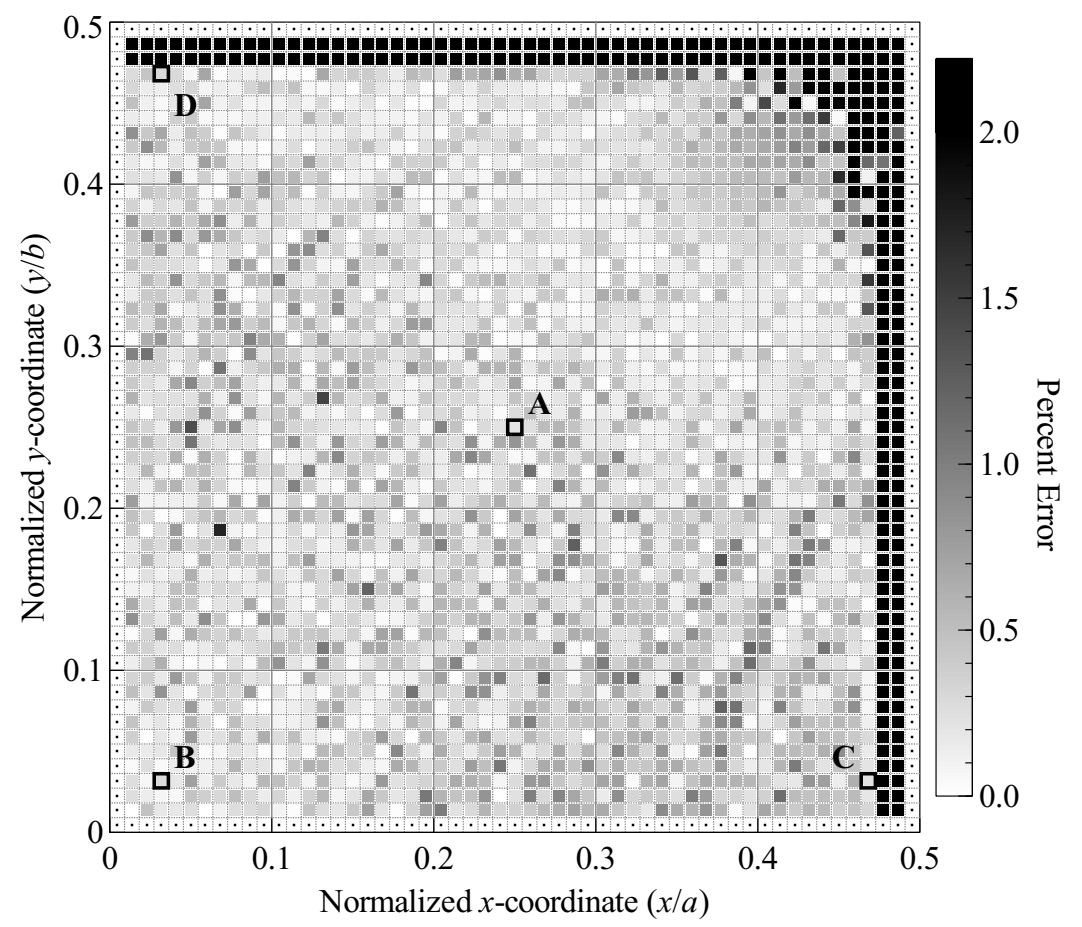

(b) Error for $55 \times 55$ mesh.

Figure 5.20: Error in recovered $\sigma_{z z}$ at top surface for all elements, varying mesh density. 
deviation of $2 \%$. Error above $2 \%$ is shown as black, regardless of its magnitude.

The results of Figure 5.20a show that the stress recovery in the majority of the plate domain produces accurate results, while elements near the supported edges of the plate produce rather inaccurate results. In fact, error for elements near the $x=a / 2, y=b / 2$ corner of the plate reaches values of greater than $100 \%$.

In an attempt to alleviate the error near the supported edges of the plate, the 55-by-55 described previously is used for recovering stresses over the whole quarter-model domain. The results are shown in Figure 5.20b, with the same data representation as Figure 5.20a. Additionally, the four points which have been previously examined are outlined for reference. With the refined mesh, a portion of elements near the supported edges still produce quite inaccurate results for the stress recovery procedure. However, note that the band of elements with high error has narrowed alonge the edges of the plate.

\subsubsection{Accuracy with Varying Loading Distributions}

Lastly, the accuracy of the stress recovery procedure to a non-uniformly distributed traction loading is determined. In place of the uniform load, a bi-harmonic distributed load described by

$$
q(x, y)=q_{0} \cdot \cos \left(\frac{x \pi}{a}\right) \cdot \cos \left(\frac{y \pi}{b}\right)
$$

is applied to the top surface of the plate, and the recovery procedure is applied to all the elements in the quarter-model domain (excluding those on the model boundary). The central plate deflection is kept at one plate thickness $(w / H \approx 1)$. To verify that the recovered boundary condition of $\sigma_{z z}$ is recovered, Figure 5.21a shows the distribution of the recovered $\sigma_{z z}$ at the top surface of the laminate. A bi-harmonic variation in the gray-scale value is expected and, as shown in Figure 5.21a, is approximately achieved. The error of the recovered $\sigma_{z z}$ at the top surface from the known traction boundary condition is shown in 


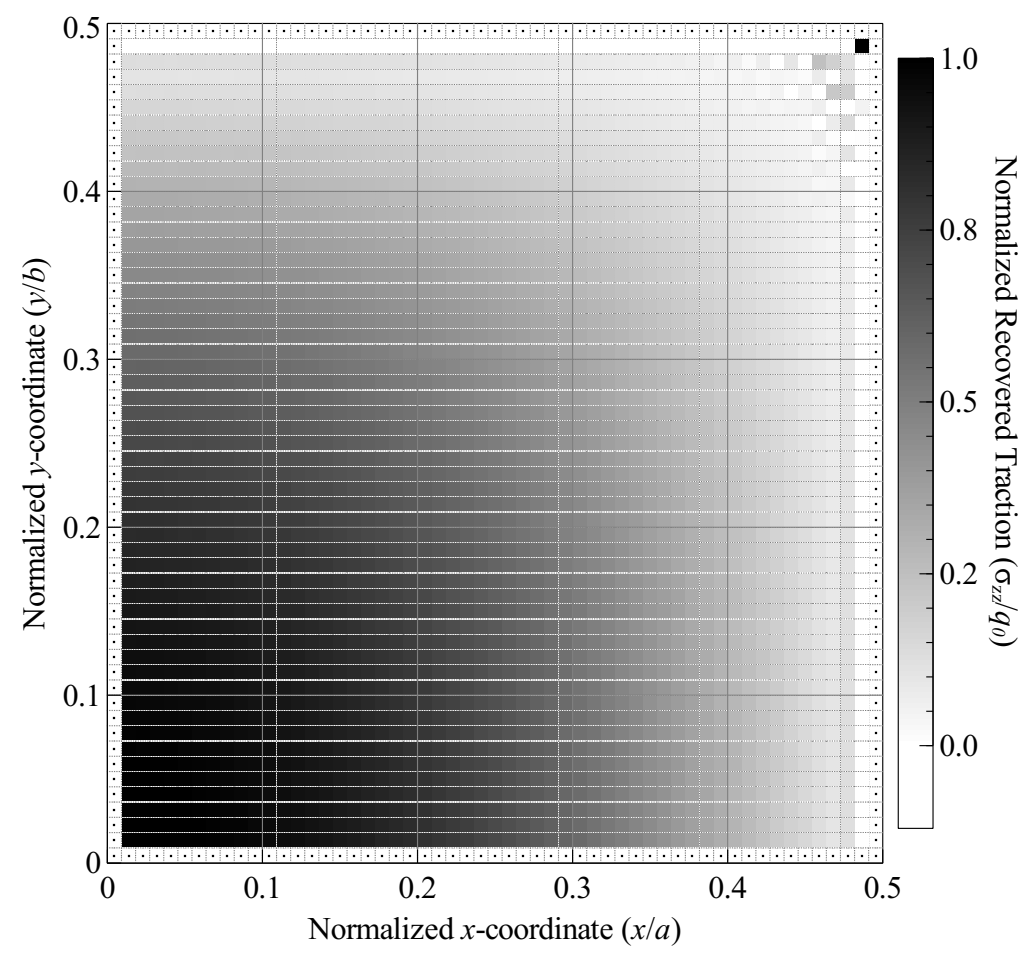

(a) Value of recovered $\sigma_{z z}$.

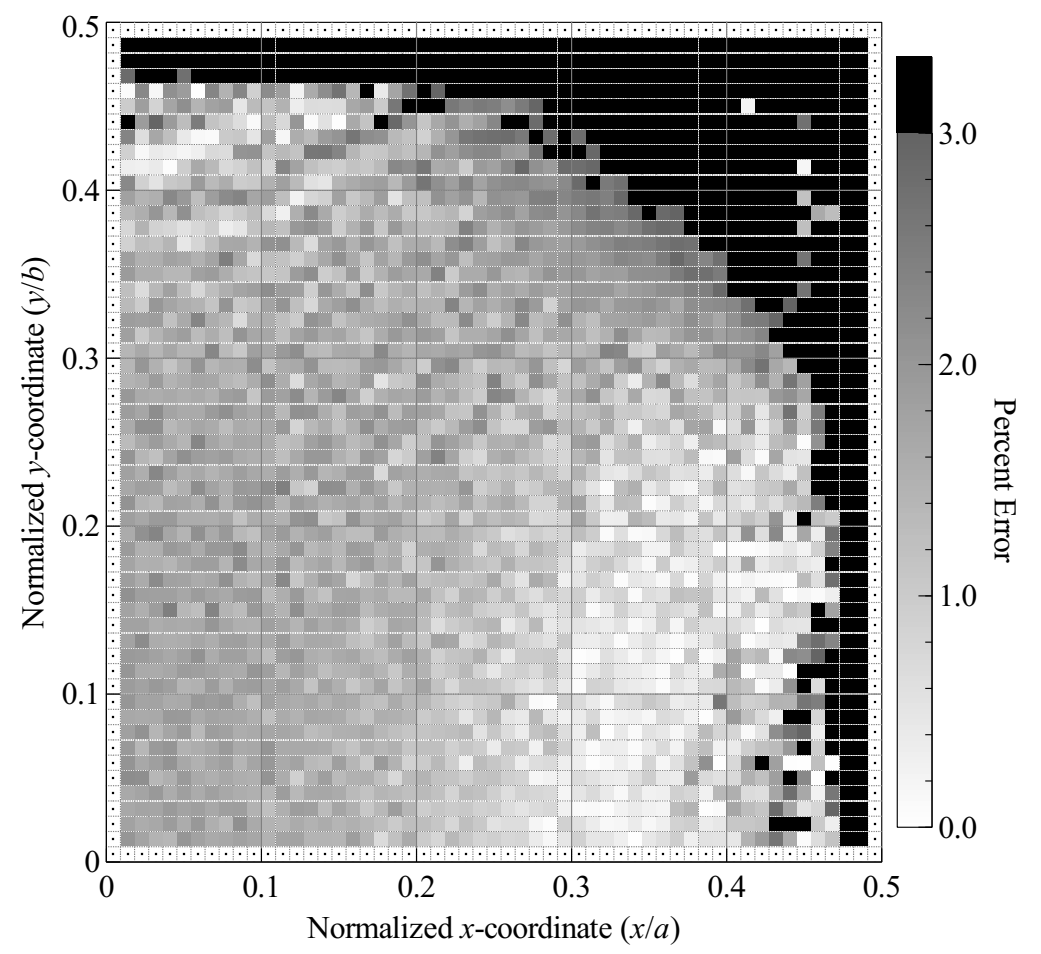

(b) Error.

Figure 5.21: Value and error of recovered $\sigma_{z z}$ for all elements under biharmonic transverse loading. 
Figure 5.21b, with the same representation as in Figure 5.20, except that the scale has been increased to $3 \%$. Most of the domain again shows an accurate recovery of the transverse normal stress, with elements near the supported edges of the plate producing high error. Note that in addition to the fact that the elements in error are near a supported edge, the value of the applied traction at the top surface decreases toward the supported edges. While the absolute error may remain the same, the percent error will necessarily increase.

\subsection{Conclusions}

The work in this chapter has demonstrated the accuracy of the developed geometrically nonlinear stress recovery procedure. The scheme was tested on a finite plate undergoing large deflections under the influence of a transverse normal pressure load. Transverse stress distributions at several points in the plate, with varying magnitudes of stresses and stress gradients, were inspected. The transverse normal stress in all cases closely matched results from the benchmark three-dimensional finite element solution. Transverse shear stresses, when not small compared to the other two transverse stresses, also showed good agreement with the benchmark solution. In all cases, the terms contained in the geometrically nonlinear equilibrium equations as presented by Byun and Kapania [34] are sufficient for the accurate recovery of the transverse stresses.

Additionally, the accuracy of the stress recovery procedure was demonstrated with various loadings and plate geometry. With plate span-ratios $a / H$ ranging from 15 to 833, error in the recovered top surface normal traction was less than 3.5\%. With plate center deflections of up to three plate thicknesses, error in the recovered top surface normal traction was less

than $6.5 \%$. Finally, the accuracy of the stress recovery procedure was good throughout the entire domain of the plate, excepting near the supported edges. Nonetheless, with mesh refinement, the width of this region was shown to decrease. 


\section{Chapter 6}

\section{Stress Recovery}

\section{Considering Inertial Effects}

The effects of considering the inertial terms of the equilibrium equations in the geometrically nonlinear stress recovery procedure are now investigated. The square, simply-supported plate of Chapter 5 is loaded with a uniform distributed time-varying load, rather than the previously quasi-static load. Prior to considering the results of the recover procedure in two model dynamic problems, the qualitative change expected with the inclusion of the body force terms are discussed. Then, the dynamic response of the $\left[0^{\circ} / 90^{\circ}\right]_{2 S}$ eight-layer plate, whose properties are unchanged from those given in Table 4.2, and span-ratio is fixed at

$a / H=60$, is considered. The density of the material is given as $1890 \mathrm{~kg} \mathrm{~m}^{-3}$. Finally, results are presented from of the geometrically nonlinear stress recovery procedure applied to two loading cases of this specific plate: a uniformly distributed pressure load with a constant loading rate, and a uniformly distributed pressure load with a sinusoidally varying amplitude. 


\subsection{Effect of Inertial Terms on Stress Recovery}

Recall the solution of the transverse stresses given by the geometrically nonlinear stress recovery procedure in Eq. (3.12), repeated here (omitting the iteration notation):

$$
\left\{\begin{array}{c}
\tau_{x z} \\
\tau_{y z} \\
\sigma_{z z}
\end{array}\right\}=\left(\begin{array}{ccc}
1 & \omega_{z} & -\omega_{y} \\
-\omega_{z} & 1 & \omega_{x} \\
\omega_{y} & -\omega_{x} & 1
\end{array}\right)\left\{\begin{array}{l}
f_{x z} \\
f_{y z} \\
f_{z z}
\end{array}\right\}+\left\{\begin{array}{l}
\left.\tau_{x z}\right|_{z=z_{j-1}} \\
\left.\tau_{y z}\right|_{z=z_{j-1}} \\
\left.\sigma_{z z}\right|_{z=z_{j-1}}
\end{array}\right\}
$$

The variables $f_{x z}, f_{y z}$, and $f_{z z}$ were given by Eq. (3.14), here applying D'Alembert's principle from Eq. (3.3), and are given as

$$
\begin{aligned}
f_{x z} & =-\int_{z^{0}}^{z}\left(\frac{\partial}{\partial x}\left(\sigma_{x x}-\omega_{z} \tau_{x y}+\omega_{y} \tau_{x z}\right)+\frac{\partial}{\partial y}\left(\tau_{x y}-\omega_{z} \sigma_{y y}+\omega_{y} \tau_{y z}\right)-\rho a_{X}\right) \mathrm{d} z \\
f_{y z} & =-\int_{z^{0}}^{z}\left(\frac{\partial}{\partial x}\left(\omega_{z} \sigma_{x x}+\tau_{x y}-\omega_{x} \tau_{x z}\right)+\frac{\partial}{\partial y}\left(\omega_{z} \tau_{x y}+\sigma_{y y}-\omega_{x} \tau_{y z}\right)-\rho a_{Y}\right) \mathrm{d} z \\
f_{z z} & =-\int_{z^{0}}^{z}\left(\frac{\partial}{\partial x}\left(-\omega_{y} \sigma_{x x}+\omega_{x} \tau_{x y}+\tau_{x z}\right)+\frac{\partial}{\partial y}\left(-\omega_{y} \tau_{x y}+\omega_{x} \sigma_{y y}+\tau_{y z}\right)-\rho a_{Z}\right) \mathrm{d} z
\end{aligned}
$$

For plate analyses, the accelerations are constant through the thickness (that is, only given at the reference surface). The density is constant through any given layer, so the contribution of the inertial terms to $f_{x z}, f_{y z}$, and $f_{z z}$ is linear in $z$ in each layer ${ }^{1}$. Omitting the stress terms in Eq. (3.14), Eq. (3.12) is expressed as

$$
\left\{\begin{array}{c}
\tau_{x z} \\
\tau_{y z} \\
\sigma_{z z}
\end{array}\right\}=\left(\begin{array}{ccc}
1 & \omega_{z} & -\omega_{y} \\
-\omega_{z} & 1 & \omega_{x} \\
\omega_{y} & -\omega_{x} & 1
\end{array}\right)\left\{\begin{array}{l}
-\rho a_{X} z \\
-\rho a_{Y} z \\
-\rho a_{Z} z
\end{array}\right\}+\left\{\begin{array}{c}
\left.\tau_{x z}\right|_{z=z_{j-1}} \\
\left.\tau_{y z}\right|_{z=z_{j-1}} \\
\left.\sigma_{z z}\right|_{z=z_{j-1}}
\end{array}\right\}
$$

Calculations for results presented here have shown that $\omega_{x}$ and $\omega_{y}$ are on the order of $10^{-1}$, with $\omega_{z}$ orders of magnitude less. Likewise, the transverse acceleration $a_{Z}$ is orders

\footnotetext{
${ }^{1}$ That is, the inertial terms in $f_{x z}, f_{y z}$, and $f_{z z}$ can be expressed by first order polynomials.
} 
of magnitude greater than the in-plane accelerations $a_{X}$ and $a_{Y}$. Thus, a few properties of Eq. (6.2) can be noted. First, the inertial terms contribute linearly within each physical layer. Second, the acceleration in the global $Z$-direction contributes to $\tau_{x z}$ and $\tau_{y z}$ on the same order as, or even greater than, $a_{X}$ and $a_{Y}$. This contribution will be noticeable, but only if the magnitude of the transverse shear stresses is small. Finally, with the approximate magnitudes mentioned and for a laminate with constant density through the thickness, the recovered top-surface traction including inertia will differ from the value ignoring inertia by approximately $\rho a_{Z} H$. For stress recovery of a geometrically linear problem ${ }^{2}$, this is the exact difference, and the stress distribution is shown in Figure 6.1. Here the bold solid line represents the transverse normal stress distribution including inertial terms. This differs from the well-known piecewise cubic variation of the normal stress through the thickness for a quasi-static load (thin line) by the contribution of the inertial term $\int \rho a_{Z} z \mathrm{~d} z$ (dashed line).

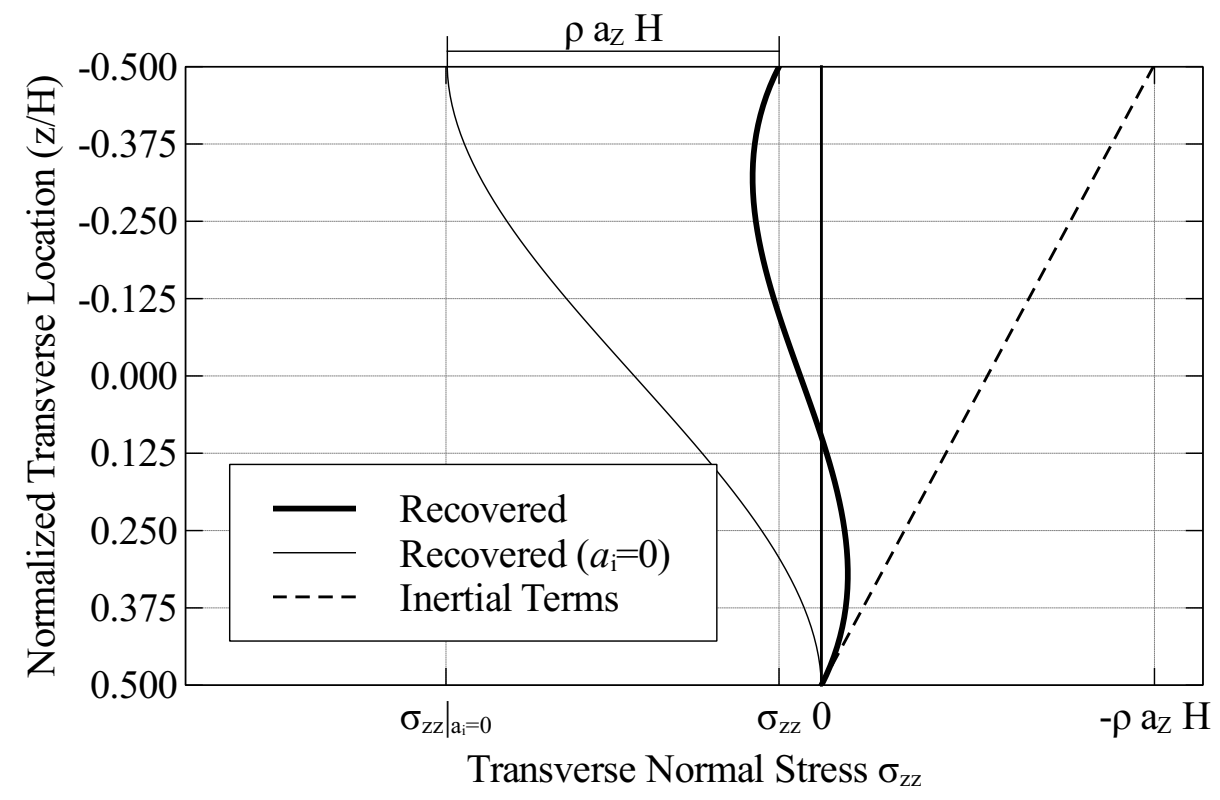

Figure 6.1: Stress recovery of linear dynamic problem including inertial terms.

\footnotetext{
${ }^{2}$ The example shown is for the same eight-ply layup discussed. The span-ratio was increased to $a / H \approx 833$ $(a=1 \mathrm{~m})$ and the undamped steady state vibratory response was found with an applied forcing frequency of $90 \%$ that of the natural frequency $\left(f=0.9 \omega_{n}\right)$. The results are given at the quarter-point at the peak plate deflection (corresponding to the peak acceleration).
} 


\subsection{Plate Dynamic Response}

For the model plate with span-ratio $a / H=60$, the first natural frequency based on classical linear vibration analysis is $1049 \mathrm{~Hz}$. However, as the plate undergoes a finite deflection, this frequency will change with plate deflection. With the tensile membrane stresses that develop due to the pinned boundary conditions, the natural frequency will increase with increasing deflection [57]. If a static uniform pressure load is applied to the plate, the dependence of the first natural frequency on the center plate deflection is depicted in Figure 6.2. (The frequency of the next three modes are shown for this same loading condition for reference.) Figure 6.2 was obtained by conducting an eigenvalue analysis at incremental load levels for the quasistatic uniformly distributed load case studied in Section 5.2. The three-dimensional finite element model from Section 5.1.2 was modified using LS-DYNA's *CONTROL_IMPLICIT_-

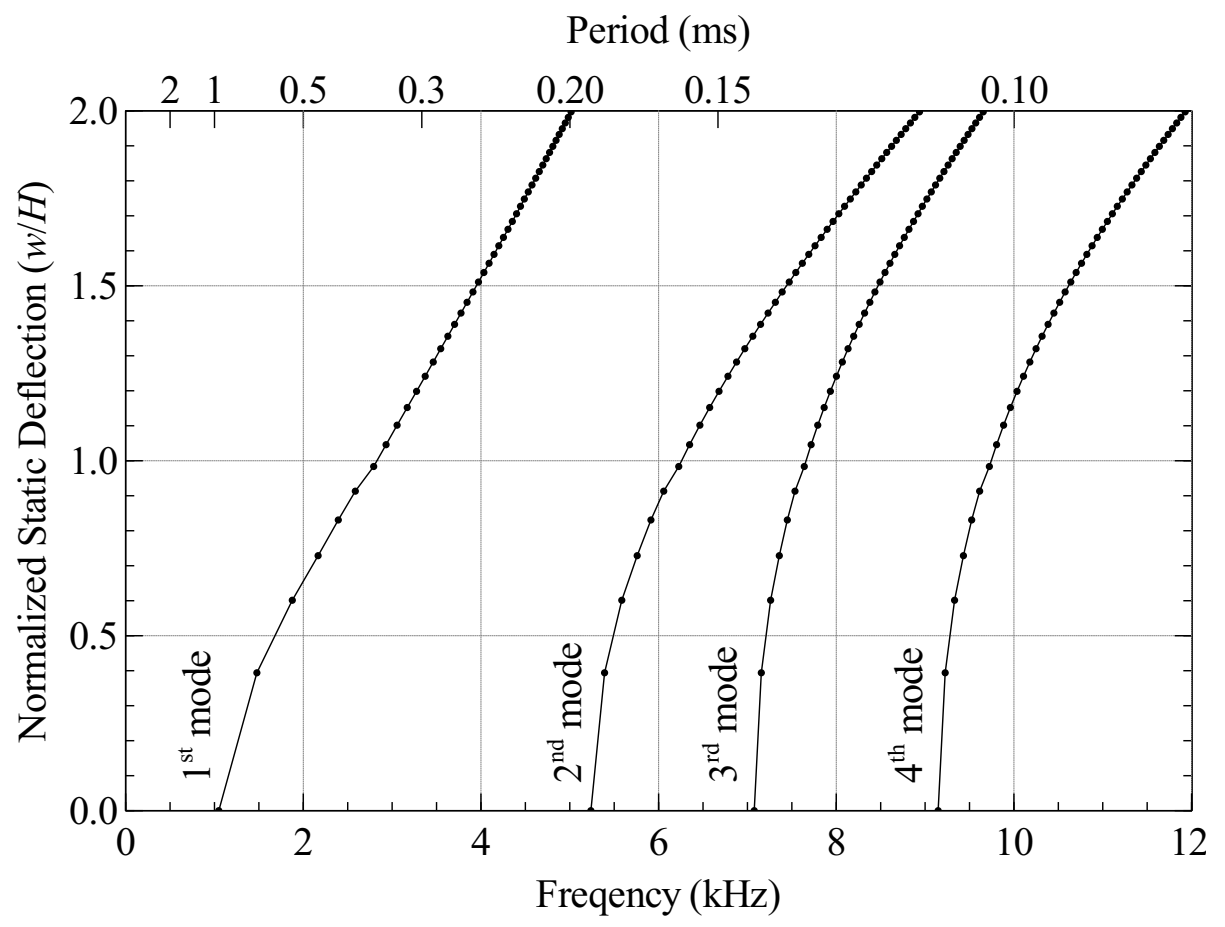

Figure 6.2: Linear natural frequency and period dependence on plate deflection due to a uniform static load. 
EIGENVALUE card with an intermittent eigenvalue analysis defined. The corresponding mode shapes for the first four modes are displayed in Figure 6.3.

\subsection{Details of Finite Element Models}

The benchmark three-dimensional finite element model for the dynamic loading cases herein is identical to that given in Section 5.1, except that LS-DYNA's explicit solver is used. For the results given, the benchmark solution is nearly converged with a rectangular mesh of 55 elements per side of the one-quarter plate model and two elements per physical layer (16 elements total through the thickness). However, the plate finite element model used for obtaining in-plane stress data for input to the geometrically nonlinear stress recovery procedure is now obtained using Abaqus [55] finite element software. The reason for the switch from LS-DYNA for the plate finite element solution is that stress results from the LS-DYNA solution did not match those from the LS-DYNA three-dimensional finite element solution. However, results from the Abaqus plate finite element solution agreed well with the LS-DYNA three-dimensional finite element solution. Details of the disagreement are given in Appendix C. The Abaqus plate finite element model has the same constraints and loading conditions as the LS-DYNA implicit plate finite element model. The rectangular mesh contains 55 elements (Abaqus type S4R shear-deformable four-noded shell elements) along each side of the one-quarter plate. The dynamic solution is obtained by explicit time integration. 


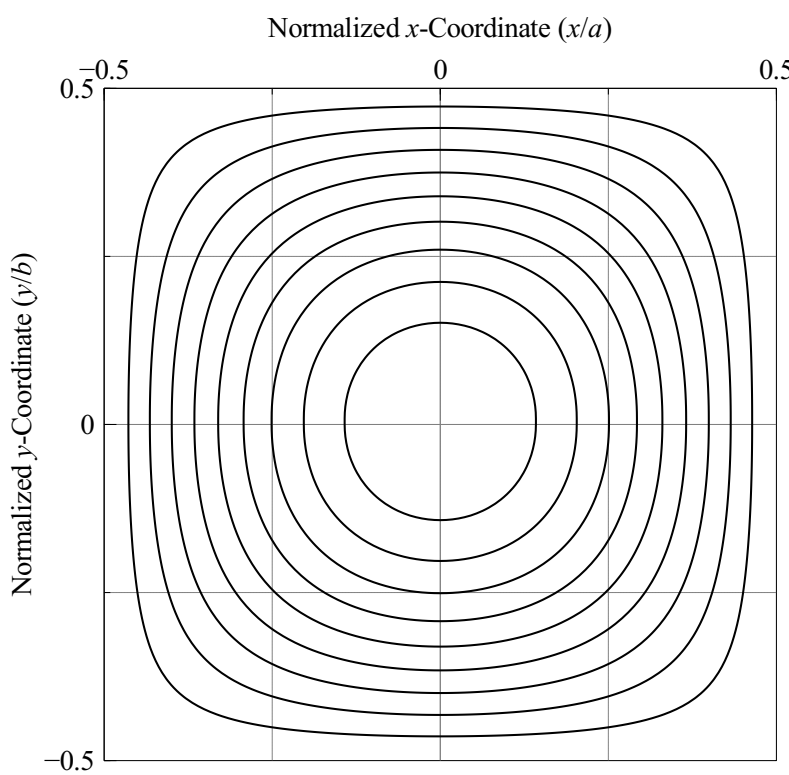

(a) First mode.

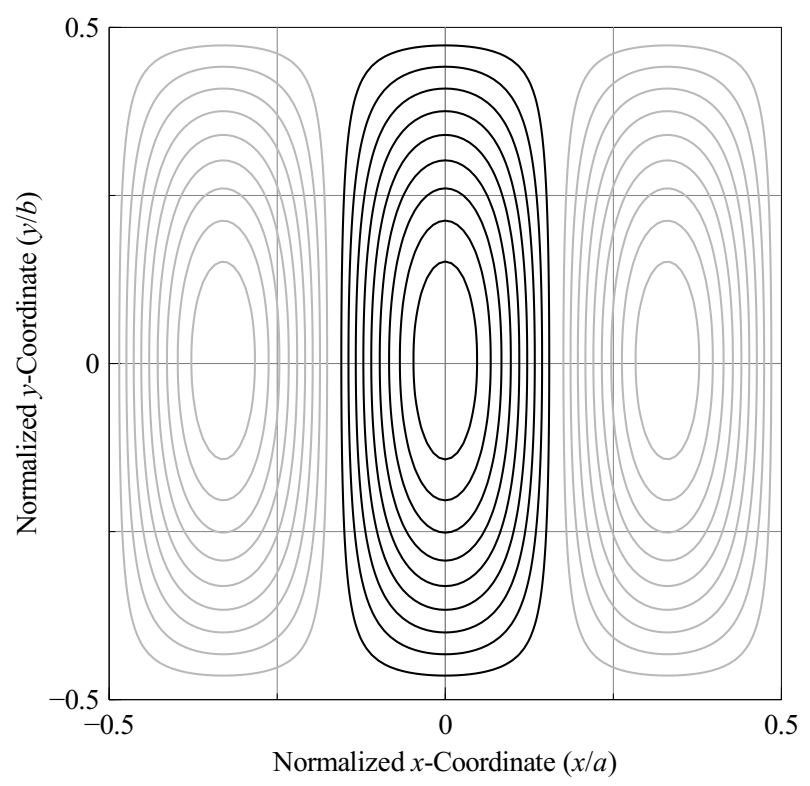

(c) Third mode.

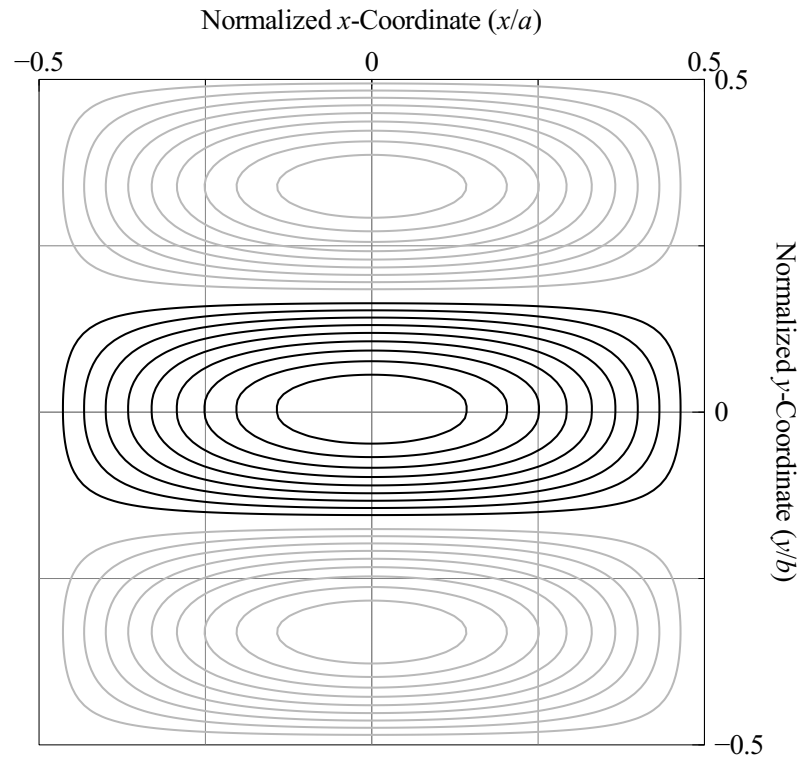

(b) Second mode.

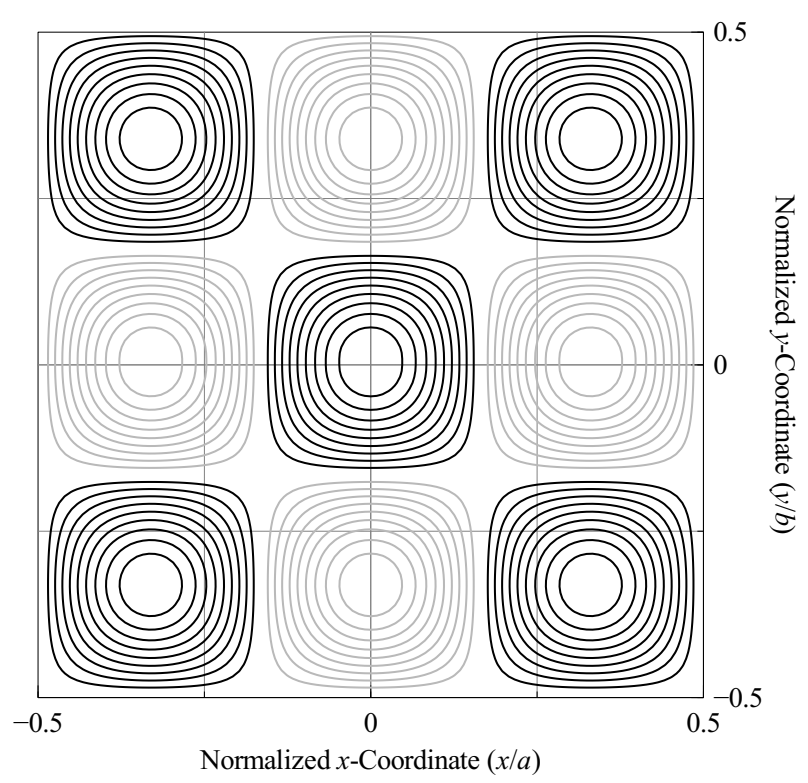

(d) Fourth mode.

Figure 6.3: First four mode shapes of dynamic plate response. 


\subsection{Results}

\subsubsection{Ramp Loading}

A ramp load is applied to the plate of interest such that

$$
q(x, y, t)=\alpha t
$$

where $\alpha$ is a constant describing the loading rate. A value of $\alpha=3 \times 10^{3} \mathrm{MPas}^{-1}$ results in the deflection and acceleration time histories of the center point of the plate as given in Figure 6.4. This loading rate was chosen because geometrically nonlinear effects become important in the plate response prior to the first oscillation. Notice that while the period of the first vibration mode is $0.954 \mathrm{~ms}$ in the undeformed configuration, the period of oscillation of the plate is about $0.2 \mathrm{~ms}$ in the deformed configuration. This value matches the period of the first mode at a center plate deflection of two plate thicknesses, as shown in Figure 6.2.

Consider the accuracy of the stress recovery procedure in predicting the transverse stresses at the quarter-point at $t=0.28 \mathrm{~ms}$. At this time, the nonlinearities are large $(w / H \approx 1.7$ at the center), but the accelerations, and, thus, inertial terms, are relatively small. Results are shown in Figure 6.5, with the same plot organization as discussed in Section 5.2.2. However, an additional thin line represents the distribution predicted by the stress recovery procedure ignoring inertial effects. The effect of neglecting acceleration in the recovery of the transverse shear stresses is virtually unnoticeable. Furthermore, neglecting nonlinear terms in the recovery procedure produces distributions which agree slightly better with the three-dimensional solution compared to including these terms.

The distribution of the transverse normal stress (Figure 6.5c) agrees slightly better when including inertial terms than when ignoring them, even for the relatively low value of acceleration at $t=0.28 \mathrm{~ms}$. The recovered transverse normal stress at the top surface is noticeably offset from the known traction, though the error is small at $3.1 \%$. 


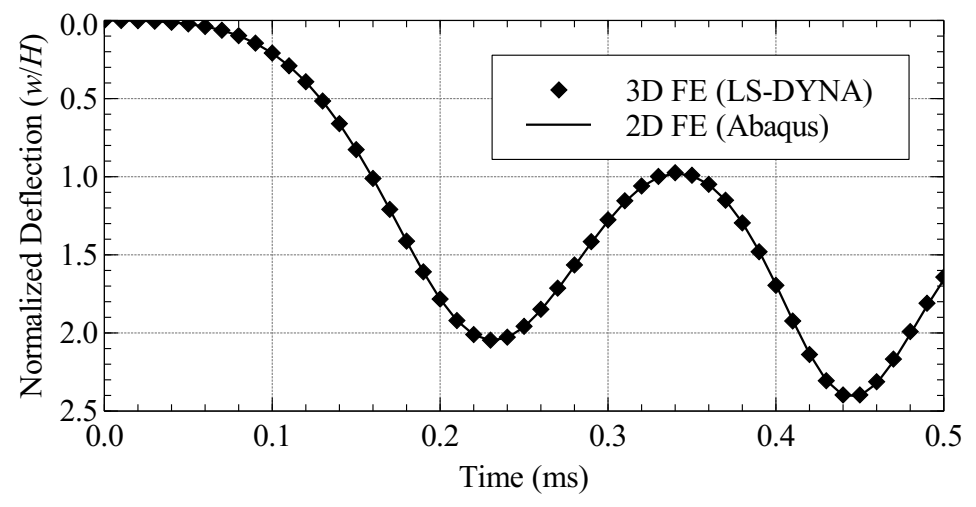

(a) Deflection.

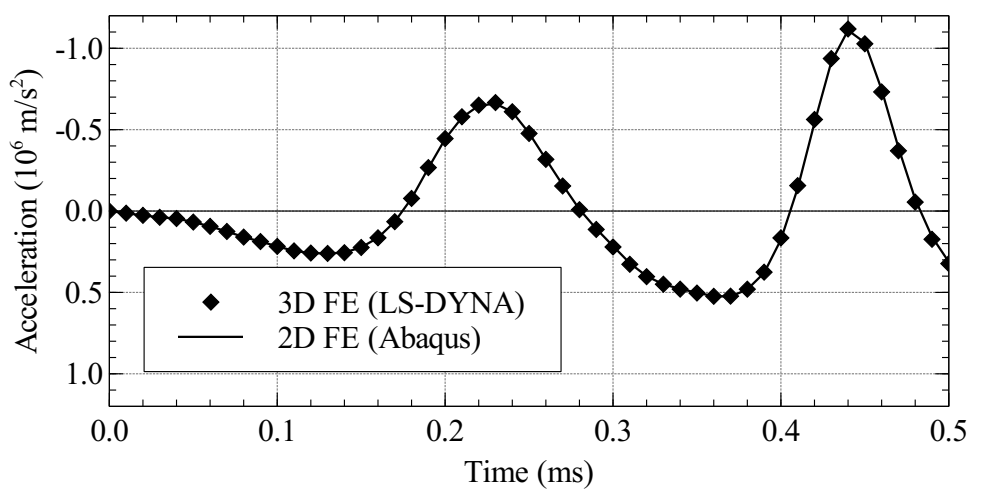

(b) Acceleration.

Figure 6.4: Kinematics of ramp loading at plate center point $x=y=0$.

The contribution of individual terms of the equilibrium equations shown in Figure 6.5d shows that, for the shear stresses, the equilibrium equations given by Byun and Kapania [34] are sufficient for an accurate representation. Furthermore, including only the terms of Byun and Kapania (in addition to the inertial term) gives a distribution that differs by only $0.1 \%$ from the recovery including all the terms.

Attention now turns toward recovering the transverse stresses at $t=0.22 \mathrm{~ms}$, where the plate deflection is slightly larger and the transverse acceleration is near a peak value. The resulting distributions are shown in Figure 6.6. The transverse shear stresses recovered by the geometrically nonlinear procedure now show less agreement with the three-dimensional solution, although the magnitudes of these stresses are a fraction of the magnitude of the 


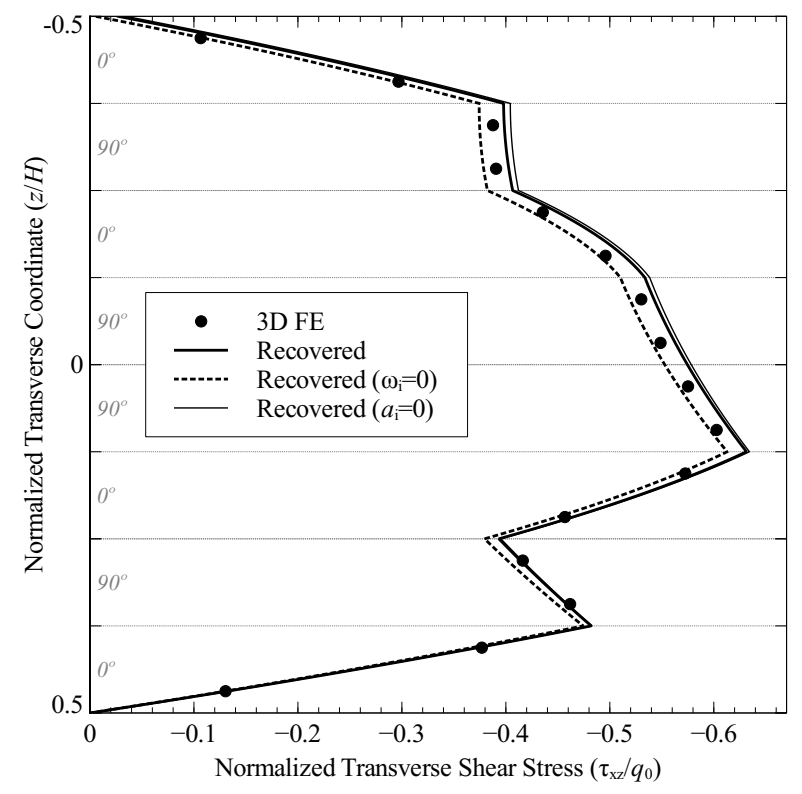

(a) Transverse shear stress $\tau_{x z}$.

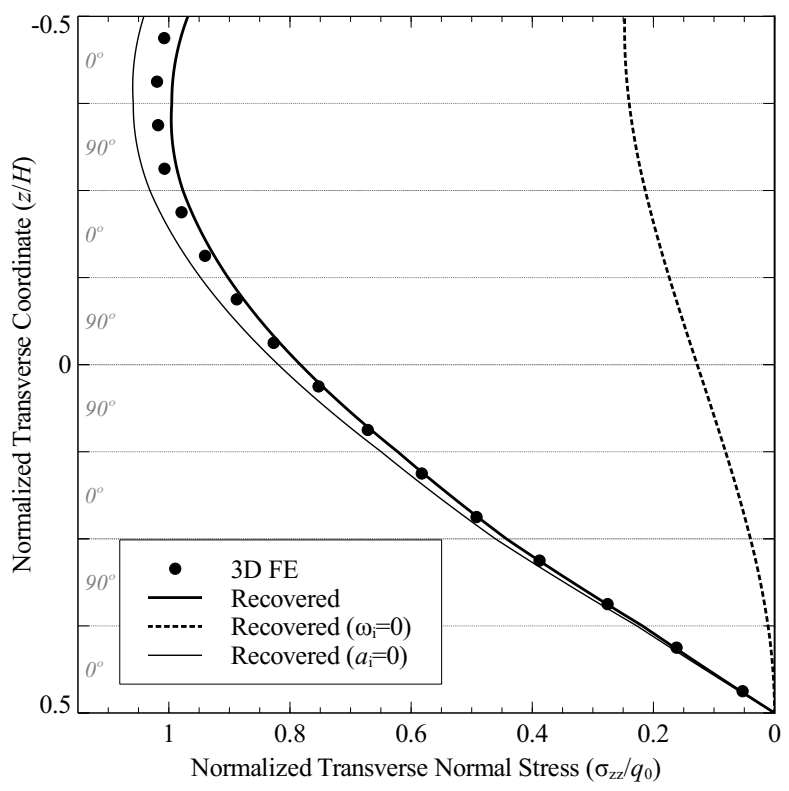

(c) Transverse normal stress $\sigma_{z z}$.

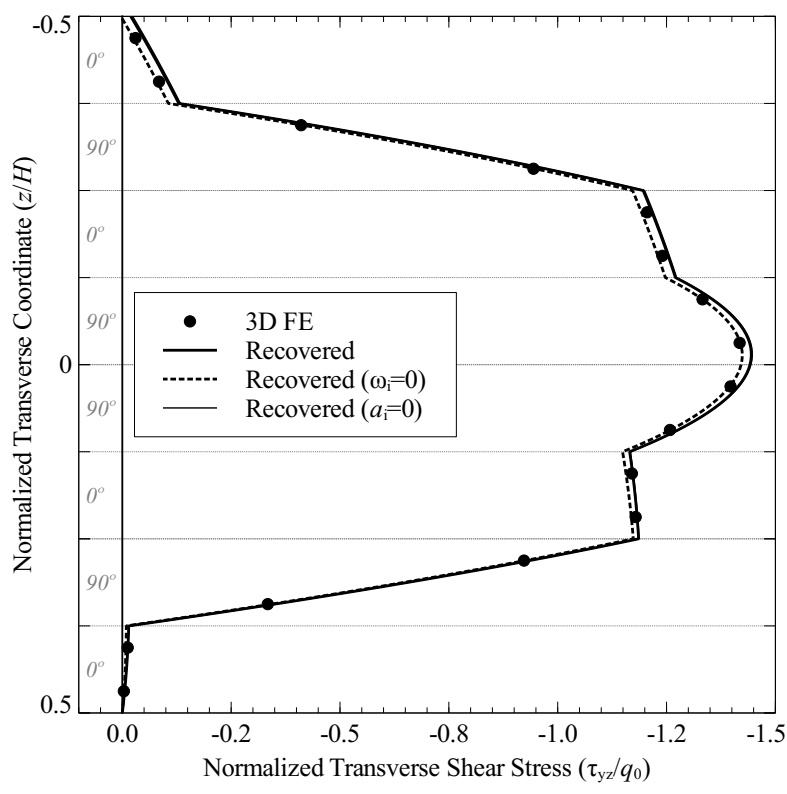

(b) Transverse shear stress $\tau_{y z}$.

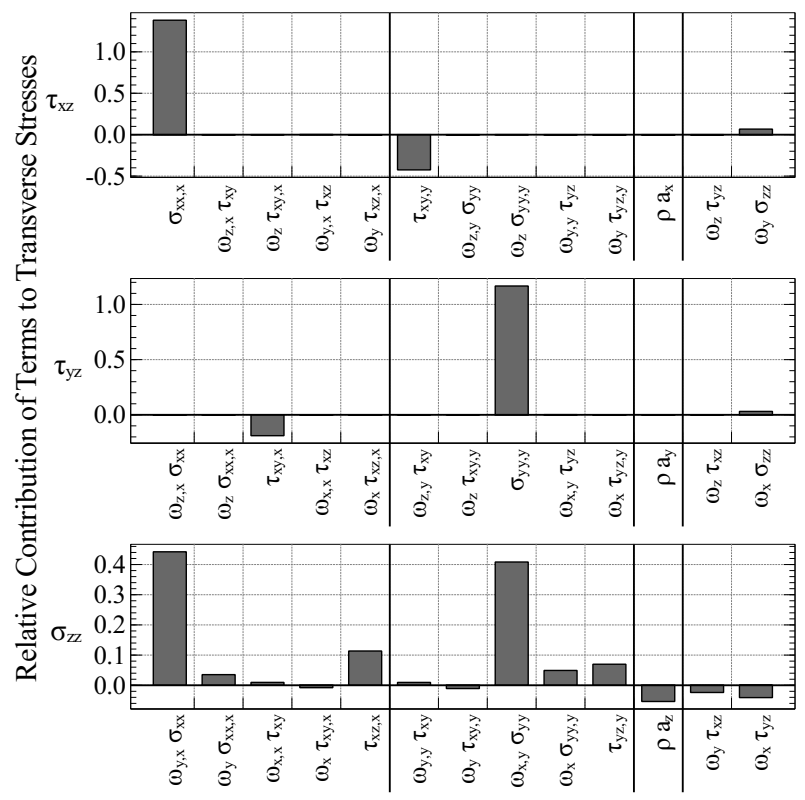

(d) Contributions of terms of Table 3.1.

Figure 6.5: Results of stress recovery at Point A. 


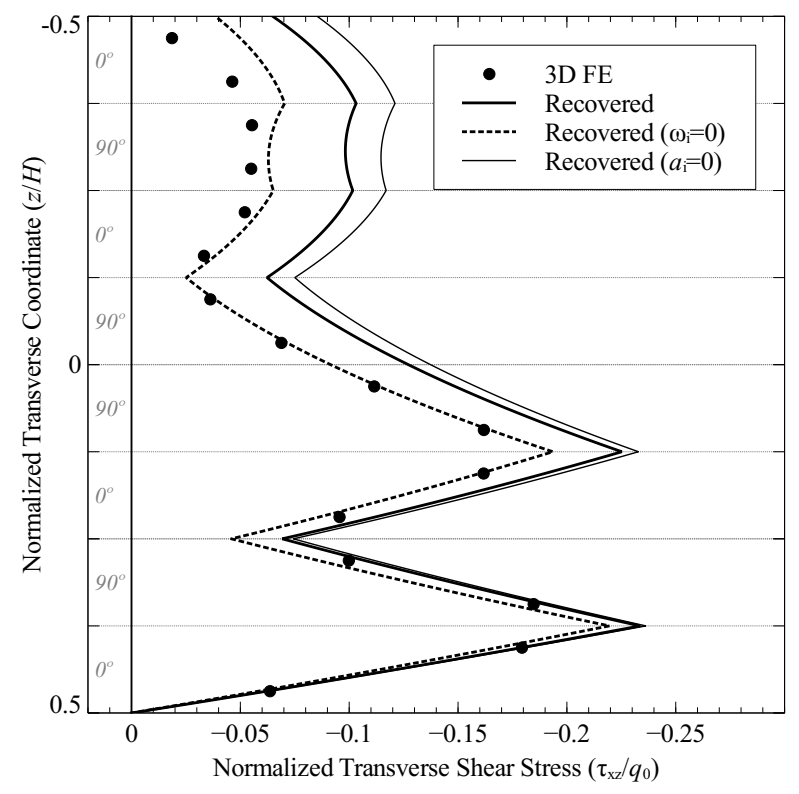

(a) Transverse shear stress $\tau_{x z}$.

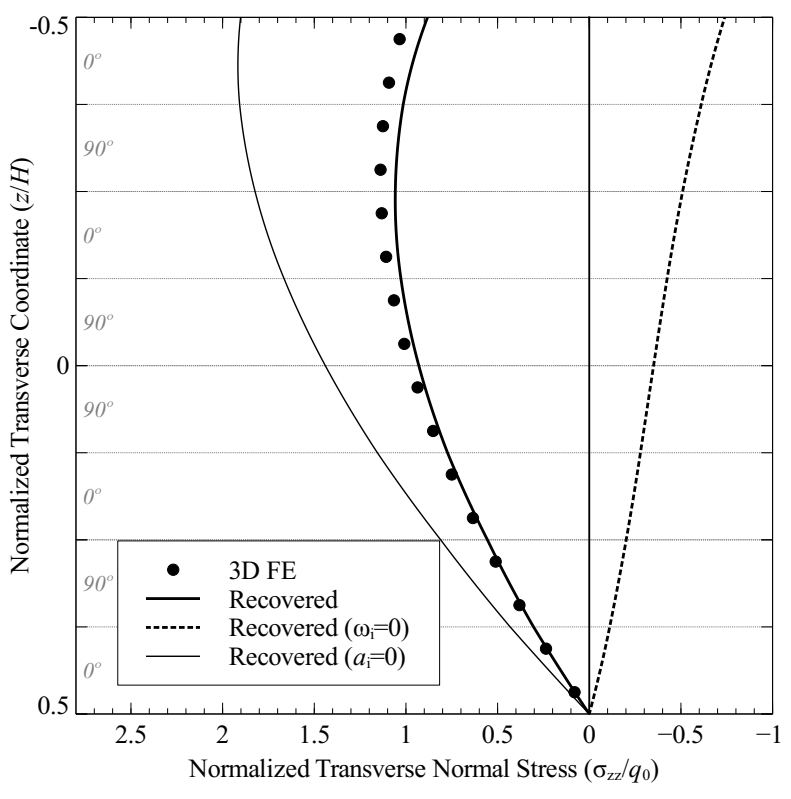

(c) Transverse normal stress $\sigma_{z z}$.

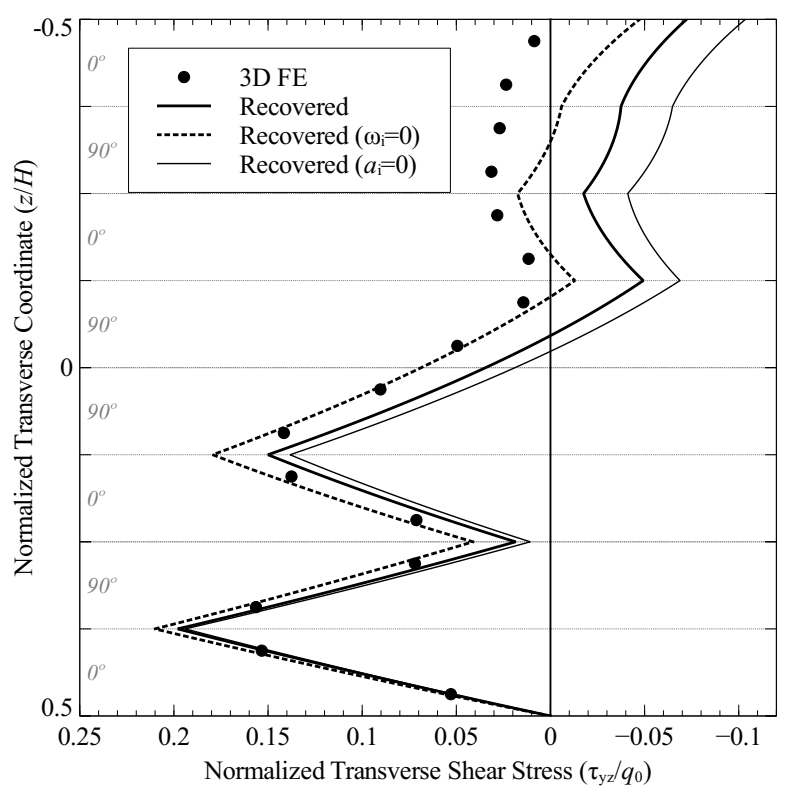

(b) Transverse shear stress $\tau_{y z}$.

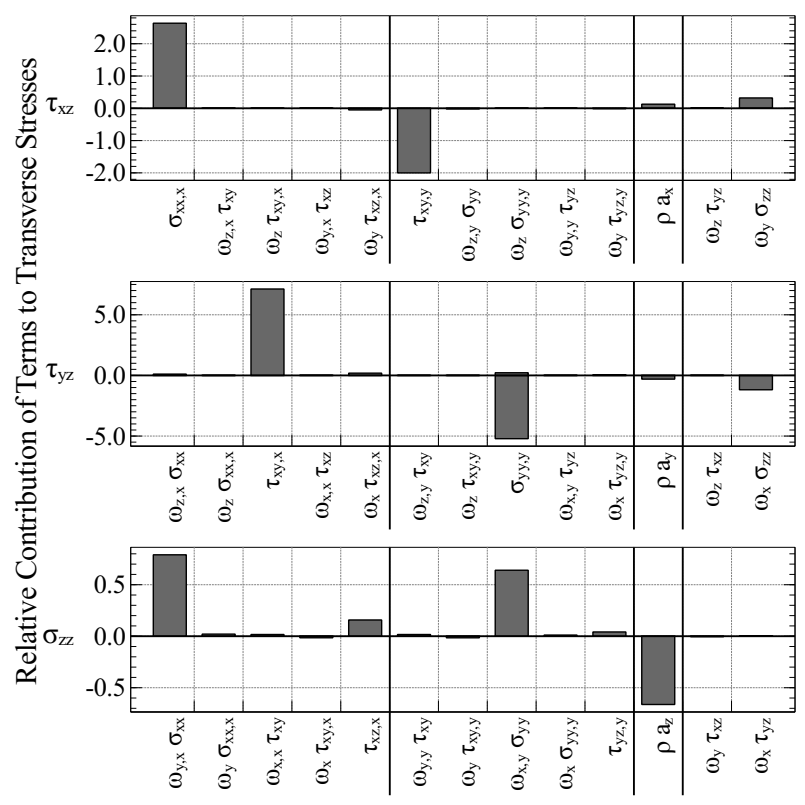

(d) Contributions of terms of Table 3.1.

Figure 6.6: Results of stress recovery at Point A, the quarter-point. 
transverse normal stress. Considering the distributions of the transverse normal stress in Figure 6.6c, ignoring either the rotation or acceleration terms leads to large error in the recovery process. The error in the recovered stresses of the current procedure is $12 \%$.

\subsubsection{Sinusoidal Loading}

The second dynamic load to be considered is the harmonic pressure load given by

$$
q(x, y, t)=\beta \sin \left(\omega_{f} t\right)
$$

where $\beta$ is a constant describing the peak magnitude of the harmonic load, and $\omega_{f}$ is the forcing frequency. A forcing frequency of $90 \%$ of the natural frequency of the plate was chosen such that

$$
\omega_{f}=0.9 \cdot 1048.6 \mathrm{~Hz}=943.7 \mathrm{~Hz}
$$

A value for $\beta$ of $100 \mathrm{kPa}$ results in a peak plate center deflection of about one plate thickness. The normalized center plate deflections of the first $3.0 \mathrm{~ms}$, as predicted by a plate finite element model (using Abaqus) and a solid finite element model (using LS-DYNA), are shown in Figure 6.7. The first period of the center deflection time history (solid line) is isolated and plotted with the forcing function (dashed line) in Figure 6.8. Discrete points are shown for every $1 / 20^{\text {th }}$ of a cycle; these points are the times at which the stress recovery procedure will be applied. Smoothing has not been applied to the deflection history between these points. The response is certainly not in a steady state for the period shown. A longer time history of the plate deflection is shown in Figure 6.9a, and it is observed that after five loading cycles the response deviates slightly from that shown in Figure 6.8. After 18 cycles, a slight beating phenomenon begins to occur, and the response given from cycles 20 to 40 begins to approximately repeat itself through at least 150 cycles. The time-history of the deflection near the plate quarter-point, Point A, is shown in Figure 6.9b. Note that the response near the quarter-point corresponds to that at the plate center, except that the 


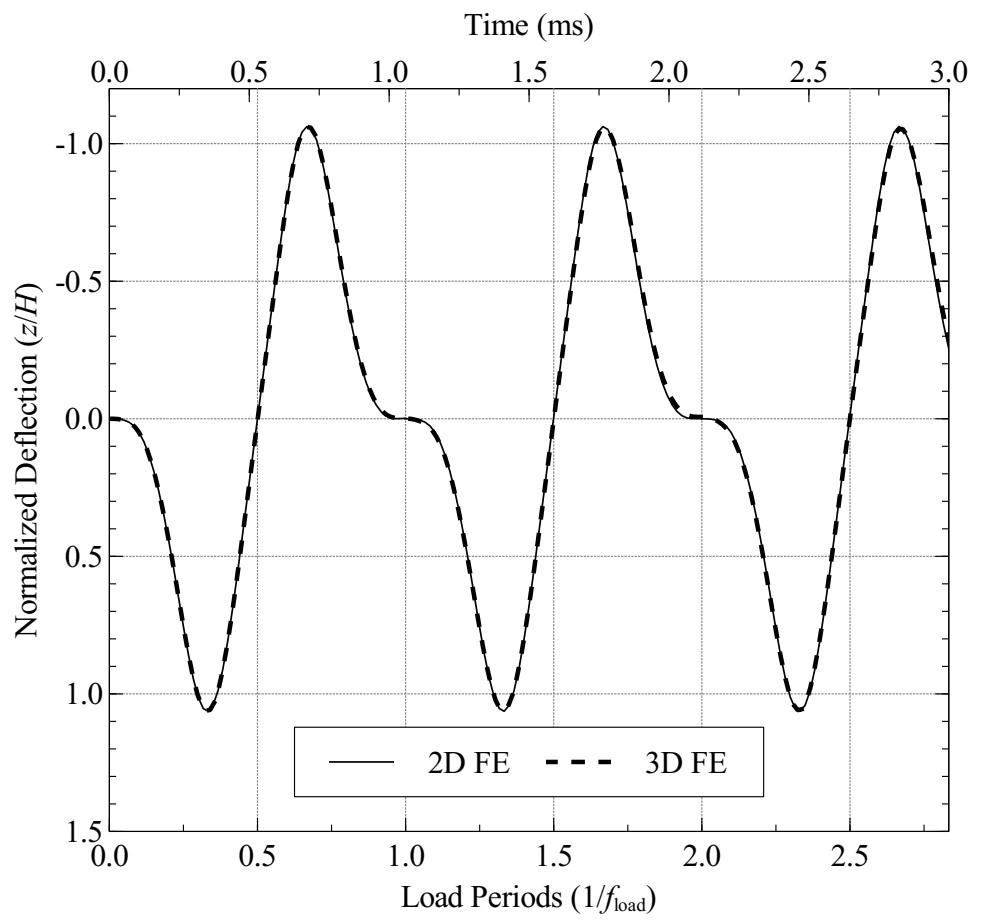

Figure 6.7: Three cycles of harmonic loading response.

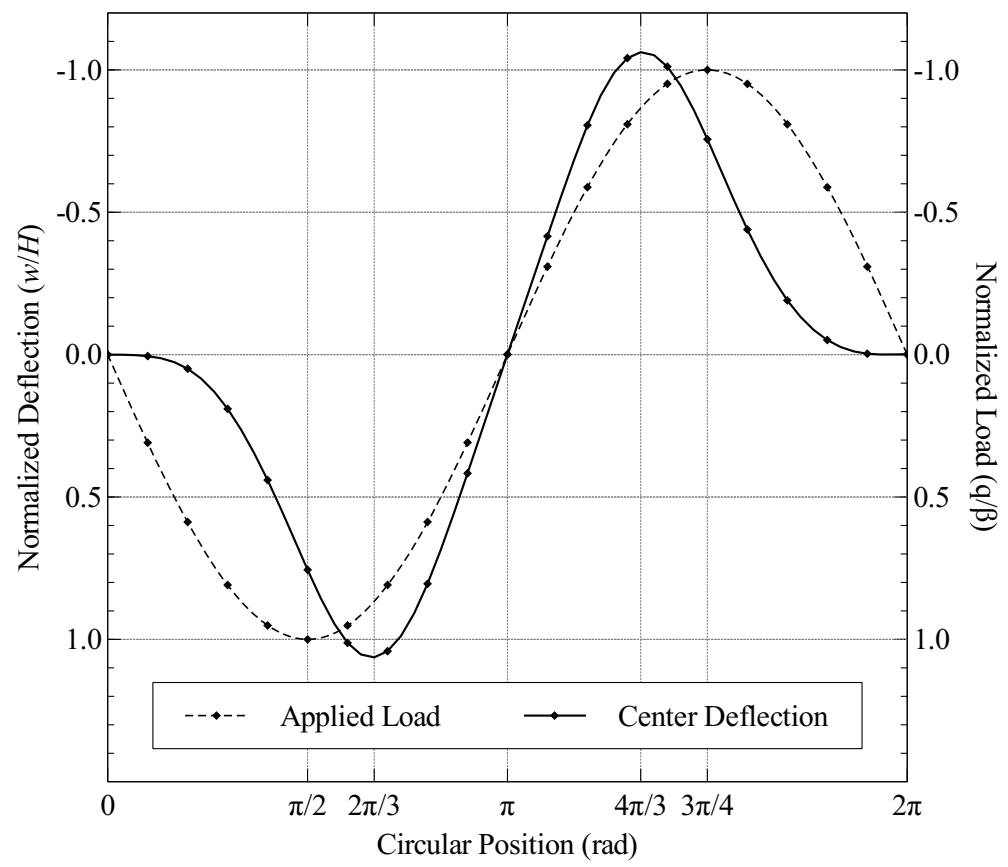

Figure 6.8: First period of harmonic loading response. 


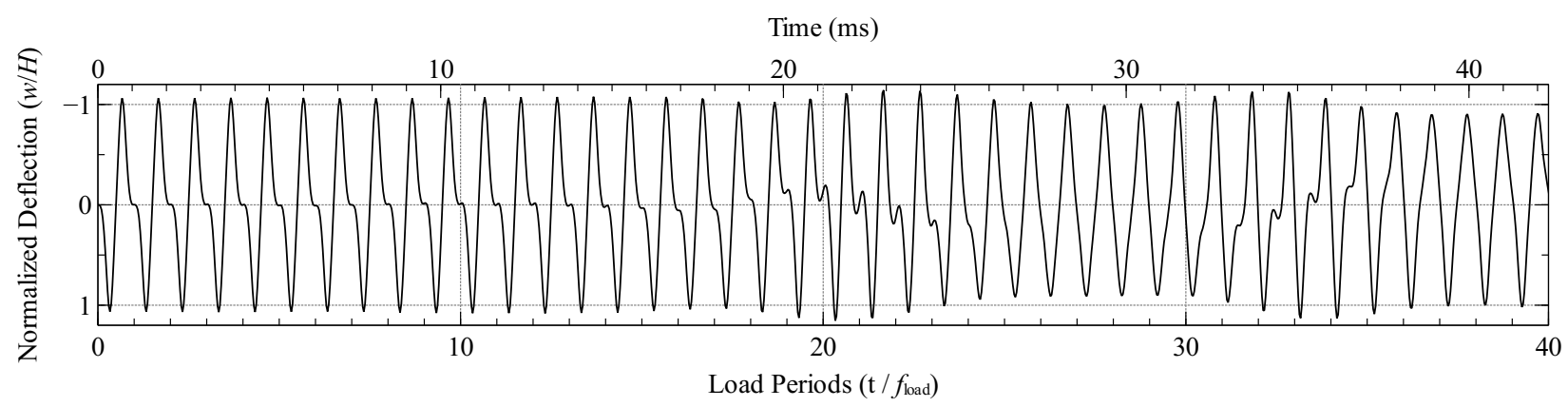

(a) Displacement history of node at center of plate $(x=y=0)$.

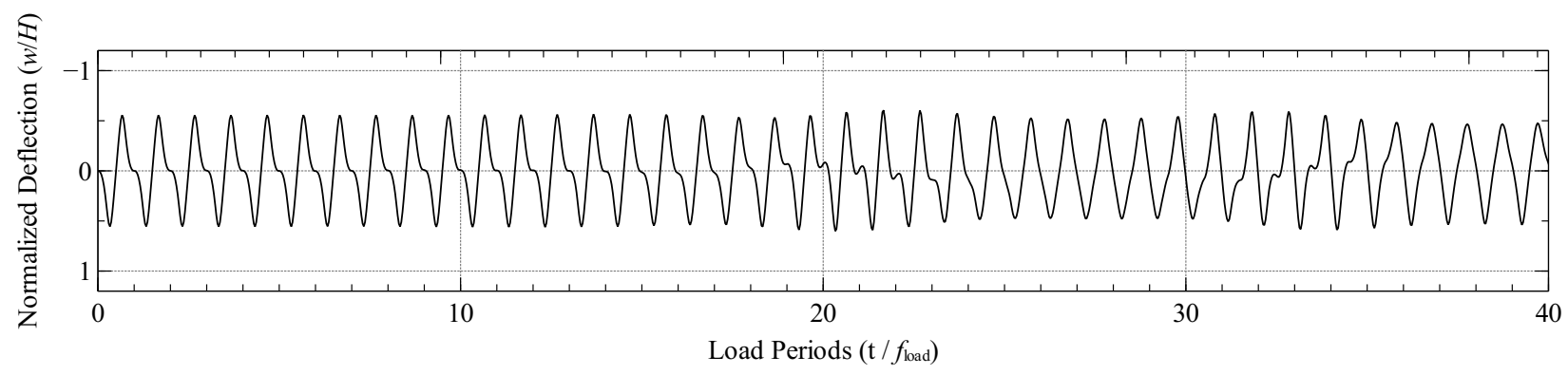

(b) Displacement history of node nearest quarter-point $(x=y \approx a / 2)$.

Figure 6.9: Plate deflection time-history.

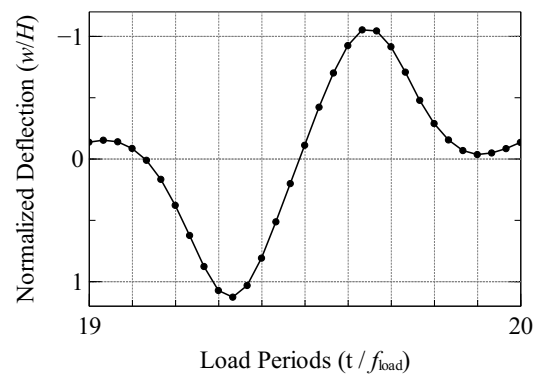

(a) $20^{\text {th }}$ cycle.

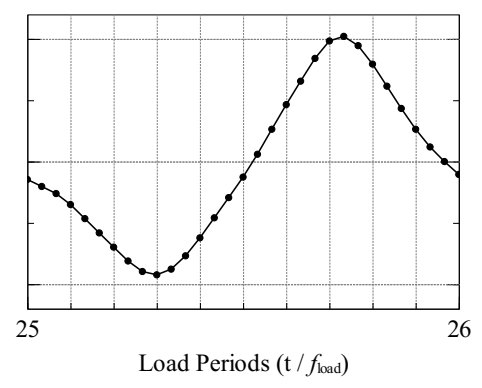

(b) $26^{\text {th }}$ cycle.

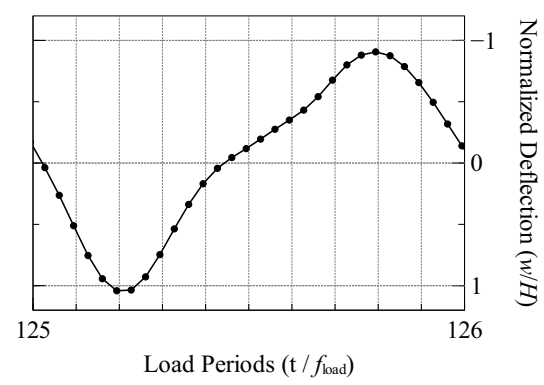

(c) $126^{\text {th }}$ cycle.

Figure 6.10: Detail of plate center deflection time-history.

deflection is approximately half that at the plate center. This serves as evidence that the beating phenomenon is not related to stress waves propagating within the plate; rather, it reflects the nonlinear vibratory response of the entire plate. Regardless of the fact that the first cycle does not represent the steady state response, it will still serve to demonstrate the accuracy of the stress recovery procedure through a variety of stress states and acceleration 
amplitudes, which can be considered periodic, at least through the first several cycles of plate response.

With the periodic response of Figure 6.8, the geometrically nonlinear stress recovery procedure considering inertial effects is applied to recover the transverse stresses at the quarter-point at 20 equal intervals (the discretized points shown in Figure 6.8) through the cycle. A time sequence of the results are shown for the complete cycle in Figure 6.11. Beginning at $t=0$, the transverse stresses $\tau_{x z}, \tau_{y z}$, and $\sigma_{z z}$ are shown from left to right with time increasing in each subsequent frame, as indicated along the right side of the figure. Recovered distributions are shown as solid lines while results obtained from the threedimensional benchmark finite element analysis are shown as discrete points. A complete one-quarter of the cycle is shown on each page; note that the last frame from each quarter cycle is repeated as the first frame for the next quarter cycle.

Through each frame of the cycle, the distribution predicted by the geometrically nonlinear stress recovery procedure shows excellent agreement with the benchmark three-dimensional solution. While the shear stresses show approximate symmetry between the two half-cycles, the form of the transverse normal stress is distinctly different in the portion of the cycle with a tensile normal traction compared to the portion in which the traction is compressive. This difference is due primarily to geometrically nonlinear effects.

\subsection{Computation Time of Stress Recovery}

A key reason for employing the stress recovery procedure is that estimates of the full stress field may be found from two-dimensional plate solutions, solutions which are typically much more computationally efficient compared to three-dimensional analyses. However, the stress recovery process takes a finite amount of time to recover the transverse stress results. It is then reasonable to question whether the time required to recover these stresses voids the time gained in moving to the two-dimensional analysis. 

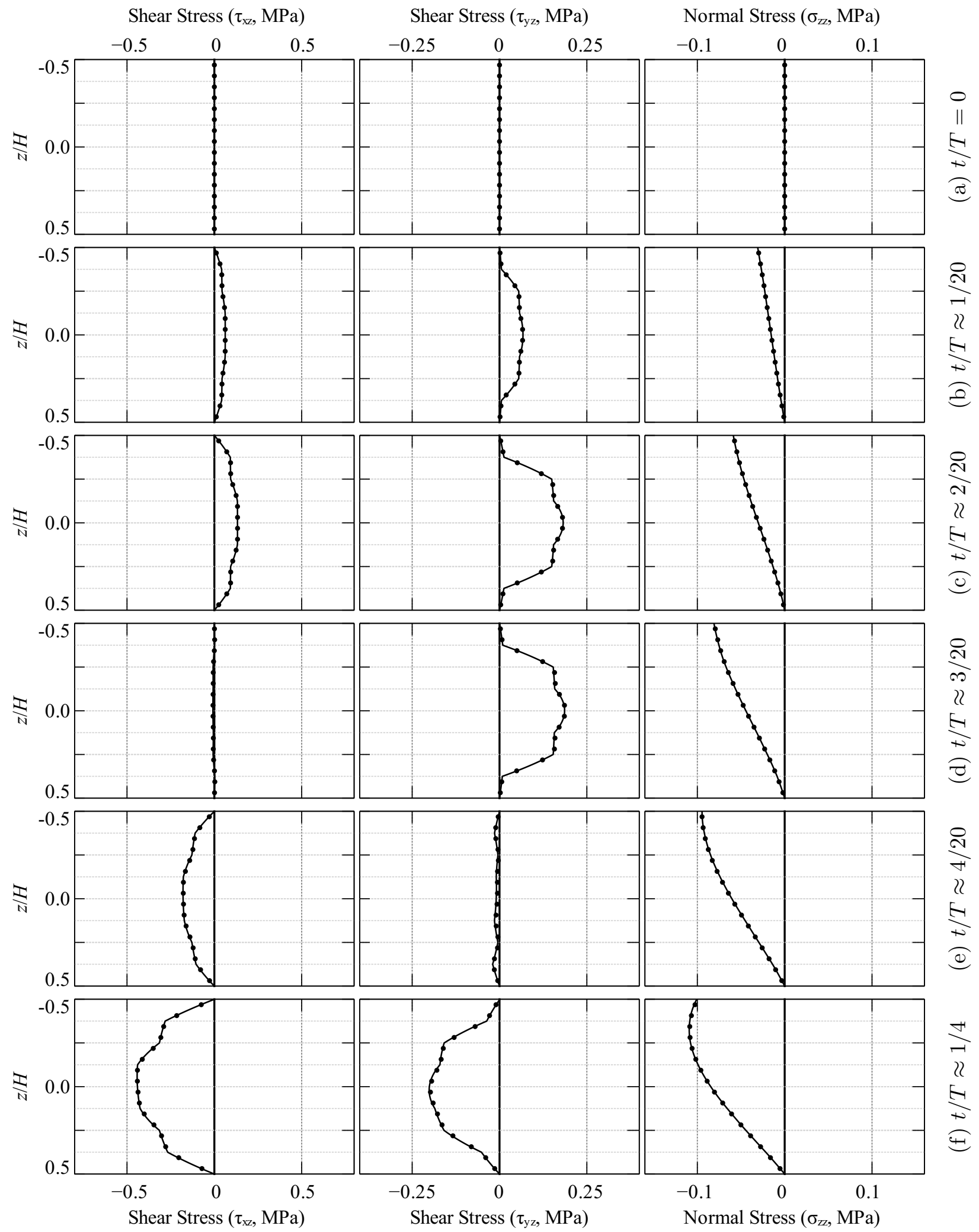

Figure 6.11: Time sequence of transverse stresses via stress recovery (solid lines) and threedimensional analysis (filled circles) for one cycle at $f=943.7 \mathrm{~Hz}$, Point A, the quarter-point. 

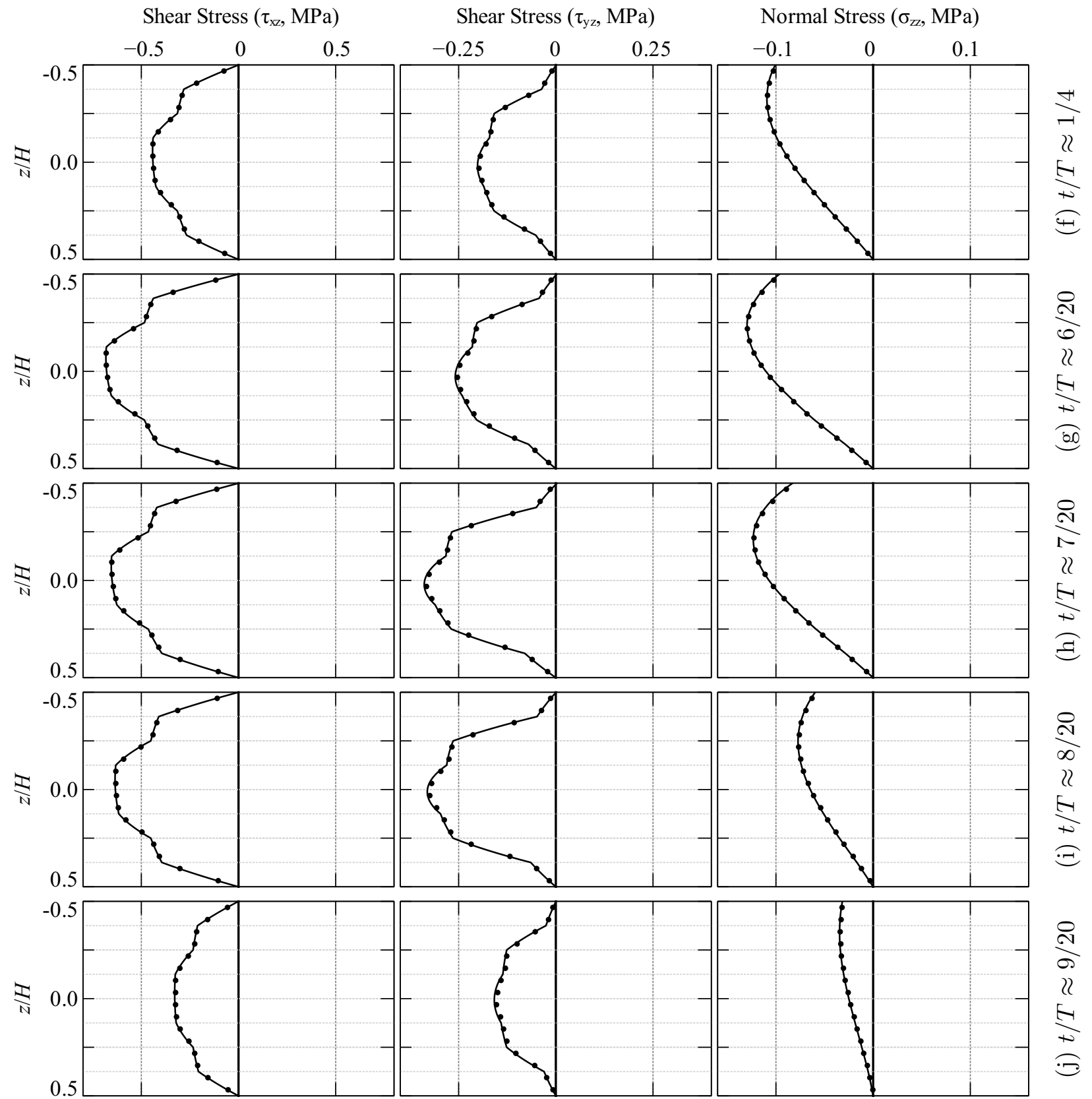

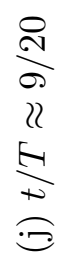
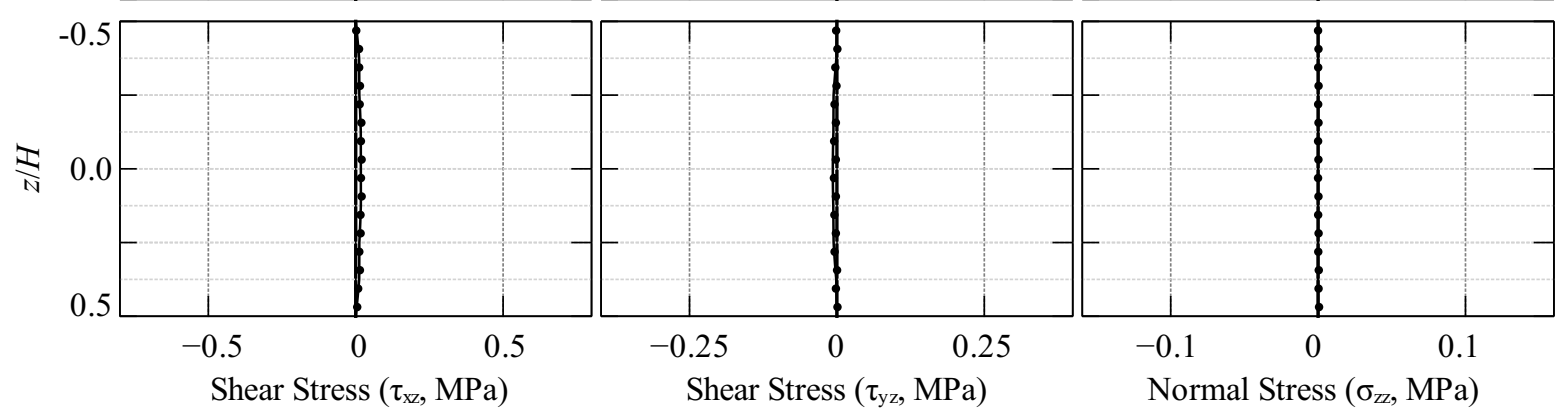

$\stackrel{N}{-1}$
$\frac{1}{-1}$
$\stackrel{+}{=}$

(cont.) Time sequence of transverse stresses via stress recovery (solid lines) and threedimensional analysis (filled circles) for one cycle at $f=943.7 \mathrm{~Hz}$, Point A, the quarter-point. 

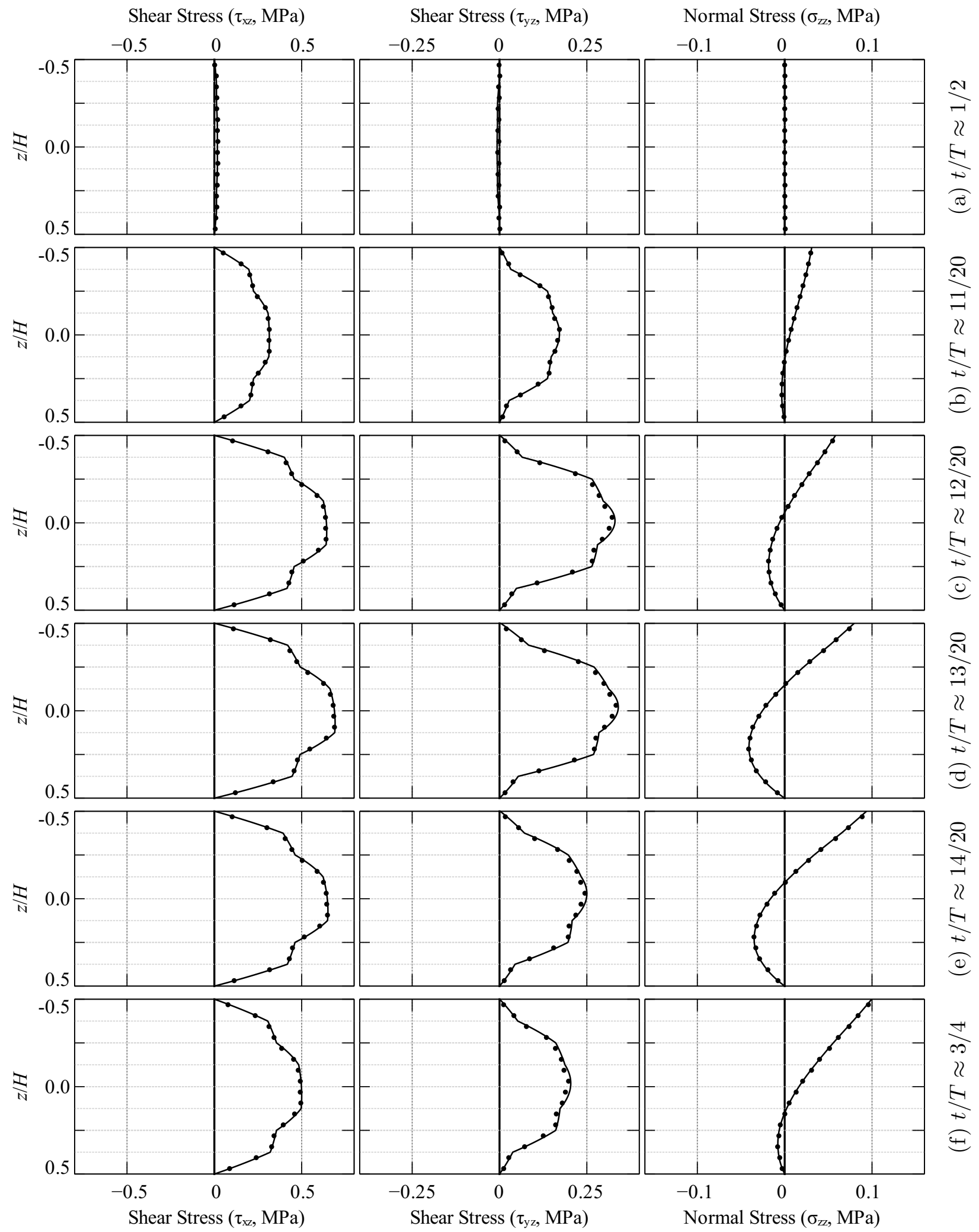

(cont.) Time sequence of transverse stresses via stress recovery (solid lines) and threedimensional analysis (filled circles) for one cycle at $f=943.7 \mathrm{~Hz}$, Point A, the quarter-point. 

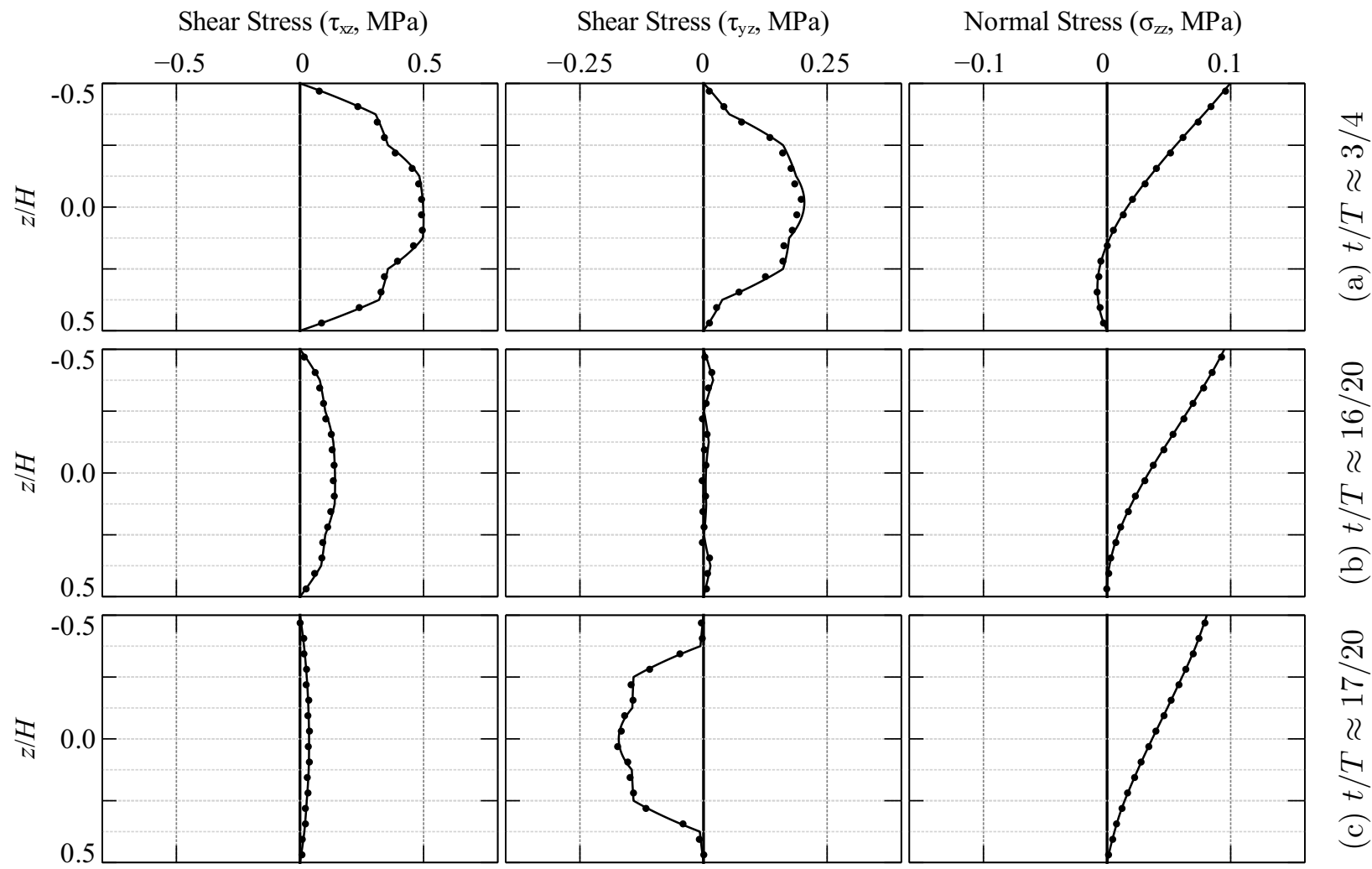

$\stackrel{0}{N}$
N
21
$\frac{1}{\infty}$
$\stackrel{0}{0}$
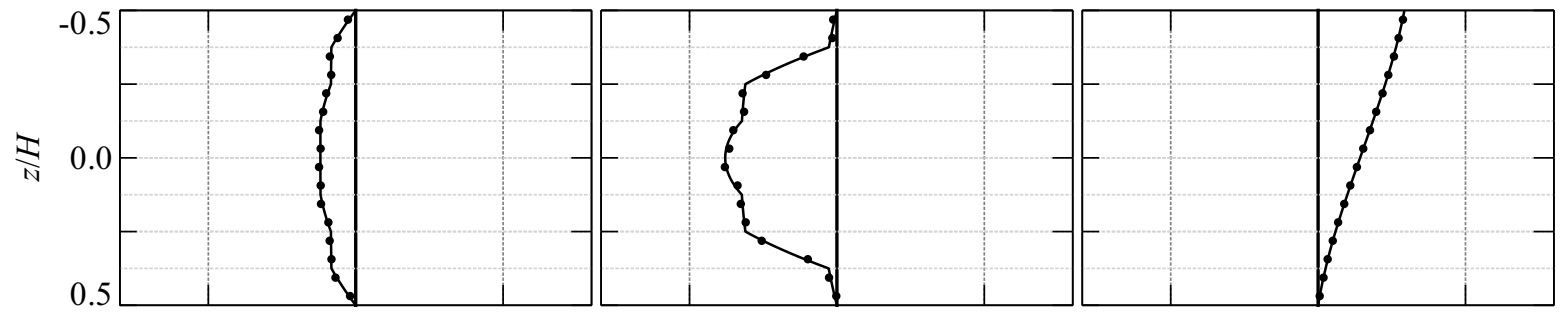

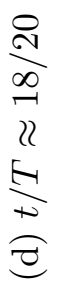
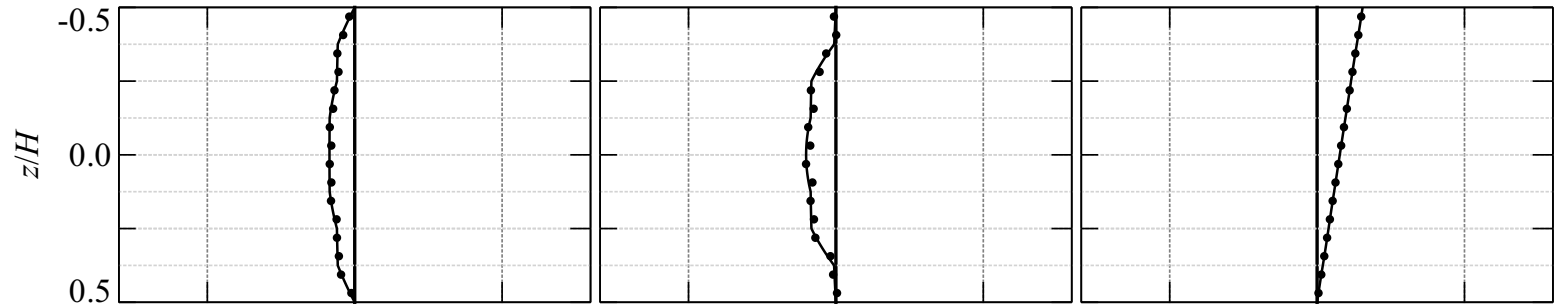

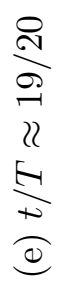
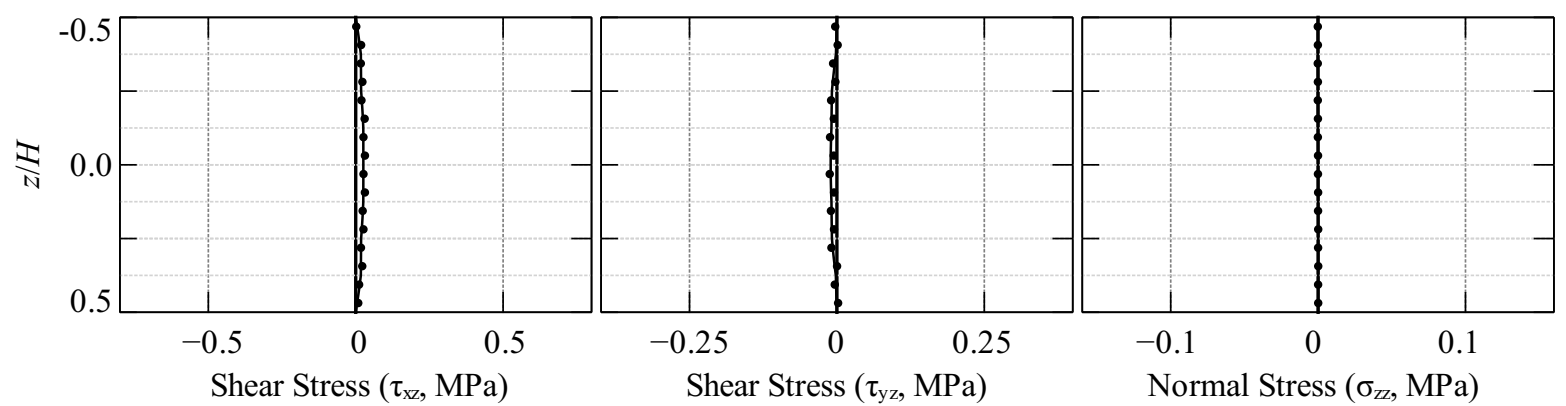

$\frac{-1}{21}$

(cont.) Time sequence of transverse stresses via stress recovery (solid lines) and threedimensional analysis (filled circles) for one cycle at $f=943.7 \mathrm{~Hz}$, Point A, the quarter-point. 
For analytical solutions in which a full three-dimensional analysis is intractable, the stress recovery process is certainly worthwhile. However, for a numerical solution such as the finite element method, the time saved by using a plate-type solution may be offset by the time required to apply the stress recovery procedure developed in Chapter 3. Here the computational time required to obtain finite element data from a plate solution and perform the stress recovery procedure on that data, is compared to the computational time required to obtain the three-dimensional finite element data.

Before proceeding, a word of caution is in order. Processing times (CPU times) of programs are known to vary greatly and depend on many factors. Among the most important are the language in which the program was written, the quality and efficiency of the program's source code, and the system on which the program is run. In order to compare run-times, these factors have been kept approximately consistent between program executions. However, while the finite element simulations run using commercially developed software, the software used to run the stress recovery process was developed in-house by an amateur programmer. Needless to say, the latter is far from being the most efficient. The comparisons then merely serve to demonstrate the efficacy of the recovery process, rather than reporting ultimate gains in computational times.

All computations take place on a dedicated compute cluster running Linux 2.6.18. Each node consists of eight Intel ${ }^{\circledR}$ Xeon ${ }^{\circledR}$ E5430 CPU's (6 MB cache, 2.66 GHz) with 32 GB shared memory. A single core is used for each program, and enough memory is provided to the LSDYNA simulations to avoid needing intermediate computations to be written to disk. For the plate finite element simulations, 1.5 GB yielded sufficient memory; for the three-dimensional finite element simulation, $10 \mathrm{~GB}$ was allocated.

The stress recovery procedure was implemented using the $\mathrm{C}++$ language and compiled using the GNU Compiler Collection [58] with the -03 optimization flag. The profiling information given below was prepared by gprof [59], with the -pg compiler flag added and a separate execution made. 
For the finite element simulations, the "Total CPU time" as reported in the d3hsp output file is used as the total computation time. For the stress recovery procedure, the time spent in user mode, as given by GNU's "time" command, is used as the total computation time. The recovery procedure was run for all elements in the 55-by-55 plate mesh (excepting the elements on the border), applying two iterations at each of 21 time points depicted in Figure 6.11.

While run times shown include the recovery of stresses at all elements (excluding boundary elements), for all 21 time points of Figure 6.11, a benefit of the stress recovery procedure is that transverse stresses may be recovered at a subset of elements, a subset of time steps, or both. Excluding areas of the plate or periods of time in which transverse stresses are not needed would dramatically reduce the computational effort compared to the results reported in Table 6.1. For example, Table 6.2 presents results for recovering the transverse stresses at the single quarter-point element for the 21 time points of Figure 6.11. In this case, the stress recovery procedure provides transverse stresses with only a small increase in computation time compared to the two-dimensional finite element anslysis.

A profile of the stress recovery implementation is presented in Table 6.3. To be noted is the large portion of time used to store and retrieve data (such as nodal coordinates, nodal displacements, element stresses, element connectivity, etc.). These data are stored primarily in the $\mathrm{C}++$ standard library's map object, which is known to not be the optimal data storage and retrieval mechanism. This is certainly an area that may be optimized in order

Table 6.1: Run time of stress recovery procedure for entire plate domain recovery.

\begin{tabular}{lcc}
\hline Process & $\begin{array}{c}\text { Two-Dimensional } \\
\text { \& Stress Recovery }\end{array}$ & Three-Dimensional \\
\hline finite element mesh & $55 \times 55$ & $55 \times 55 \times 16$ \\
\hline LS-DYNA execution & $0: 03: 08$ & $1: 57: 08$ \\
stress recovery & $0: 49: 34$ & - \\
\hline TOTAL & $0: 52: 42$ & $1: 57: 08$ \\
\hline
\end{tabular}


Table 6.2: Run time of stress recovery procedure for single element recovery.

\begin{tabular}{lcc}
\hline Process & $\begin{array}{c}\text { Two-Dimensional } \\
\& \text { Stress Recovery }\end{array}$ & Three-Dimensional \\
\hline finite element mesh & $55 \times 55$ & $55 \times 55 \times 16$ \\
\hline LS-DYNA execution & $0: 03: 08$ & $1: 57: 08$ \\
stress recovery & $0: 00: 05$ & - \\
\hline TOTAL & $0: 03: 13$ & $1: 57: 08$ \\
\hline
\end{tabular}

to see improvement in the results given in Table 6.1. Furthermore, element centroids are calculated for each calculation in which they are needed. Thus, rather than 3025 calls to calculate and store the centroids of the 3025 elements in the mesh, these routines are called 31528216 times. Meanwhile, the stress recovery routine, including calculation of stress gradients, currently requires only $5 \%$ of the total processing time.

While run time of programs provides a measure of time required to obtain solutions by various methods, additional time is required in pre-processing of simulation input and post-processing of data. Indeed, using the finite element method to obtain the mechanical response of a structure involves at least one mesh refinement process in order to verify convergence of the results. A benefit of a plate solution is that this refinement process is required only in two dimensions rather than the additional through-thickness dimension. Thus, the stress recovery procedure provides additional benefit than the 54 min suggested

Table 6.3: Profile of stress recovery implementation.

\begin{tabular}{lcc}
\hline Process description & \% Time & Total function calls \\
\hline Data storage \& retrieval & 72.34 & 1137450422 \\
Calculate element centroids & 8.06 & 31528216 \\
Stress recovery algorithm & 2.43 & 2809 \\
Calculate stress gradients & 2.43 & 14312928 \\
Find neighboring elements & 1.84 & 2755845 \\
\hline Other processes & 12.94 & 608167275 \\
\hline Total & 100.0 & 1794217495 \\
\hline
\end{tabular}


in Table 6.1. Additionally, if, for example, the interlaminar stresses were of interest in the $126^{\text {th }}$ cycle of Figure 6.10 rather than, say, the $20^{\text {th }}$ cycle, use of the three-dimensional finite element approach would require integrating in time through the $126^{\text {th }}$ cycle. This would require considerable computation time. On the other hand, integrating in time with a finite element plate analysis to reach the $126^{\text {th }}$ cycle, and then executing a stress recovery procedure, would require considerably less computation time.

\subsection{Conclusions}

This chapter has demonstrated the ability of the geometrically nonlinear stress recovery procedure to accurately predict the transverse stresses in a plate under dynamic loading conditions in which inertial terms contribute significantly to the stress distributions. For the example problems studied, error in the top surface boundary condition of the recovered transverse normal stress is always under 10\%, with those for the oscillatory loading being smaller.

Furthermore, the efficacy of the procedure with respect to computational time required compared to a full three-dimensional analysis has been demonstrated. While the current implementation of the procedure is not nearly optimized, it has been shown that the potential for time savings exists. This is especially true when considering factors such as the ability to

recover stresses in a select sub-domain of space or time, and the reduced effort of constructing and validating a full three-dimensional finite element simulation. 


\section{Chapter 7}

\section{Conclusions}

In Chapter 3, the formulation of a geometrically nonlinear stress recovery procedure was presented. This formulation is less complex than those set forth by other authors [34, 35, 40, 42, 44-47]. In particular, for in-plane stress data given by a finite element solution, the current procedure includes no correction of the in-plane response, no parametric fitting of in-plane stresses, and no additional finite element analyses.

The procedure was applied to example problems for quasi-static loading of semi-infinite (Chapter 4) and finite (Chapter 5) plates, as well as dynamic loading of finite plates (Chapter 6). For all cases, data from a three-dimensional finite element solution is provided alongside the data from the stress recovery procedure. Excellent agreement was found between the transverse normal stress distribution as estimated by the stress recovery procedure and that obtained from a three-dimensional solution. The accurate recovery of the transverse normal stress requires inclusion of the geometrically nonlinear terms (accounting for rotations of plate elements). For the example problems studied herein, estimation of the transverse shear stress distribution by the stress recovery technique compared well with the three-dimensional solution, provided the transverse shear stress was not orders of magnitude less than the transverse normal stress. However, for these problems, inclusion of the geometrically nonlinear terms does not provide a more accurate estimation of the transverse shear 
stresses compared to neglecting these terms.

Results shown for the finite plates of Chapters 5 and 6 included a study of the contribution of individual terms of the equilbrium equations. For the example problems considered, the equilibrium equations as presented by Byun and Kapania [34] included the necessary nonlinear terms for accurate recovery of the transverse stresses. However, there may exist problems to which the stress recovery procedure may be applied that require nonlinear terms which are excluded by Byun and Kapania.

The study presented in Section 5.2.4 found that the stress recovery procedure may be applied to a wide range of problems while maintaining an accurate estimation of the transverse stresses. The technique may be applied both to moderately thick and very thin plates (span-ratios of $15 \leq a / H \leq 1000$ were studied). Plate rotations of up to $0.2\left(12^{\circ}\right)$ are permitted with errors of less than $10 \%$.

Thus, the work herein has extended the state of the art of geometrically nonlinear stress recovery, as set forth in the introduction, by:

1. Developing a formulation for the geometrically nonlinear stress recovery procedure that is less complex than other implementations presented in the literature.

2. Evaluating which terms of the geometrically nonlinear equilibrium equations are necessary for accurate stress recovery, as previous works present various simplifications.

3. Comparing results from the developed stress recovery procedure, for both quasi-static and dynamic example problems, to results obtained by a benchmark three-dimensional finite element analysis.

Additionally, a software tool has been created which can process output from an LS-DYNA finite element simulation and provide recovered transverse stress results for simulations of a flat plate with a uniform mesh.

Important work yet to be completed is to extend the current geometrically nonlinear stress recovery procedure to shell structures in which curvature of the reference surface becomes 
important. In this case, the gradients of the equilibrium equations would change from the Carteasian coordinate system centered in the undeformed plate, to a curvilinear coordinate system centered in the undeformed shell. This extension would allow the stress recovery procedure to be applied to a much broader set of structural applications as compared to the flat plate examples shown.

An additional extension may be made to generalize the procedure by forgoing the restriction that rotations be moderate. In this case, Eq. (A.12) may be integrated through the thickness, with an interation scheme involving the transverse stresses and transverse strains ensuing.

The work presented here contains the necessary components required for applying the stress recovery procedure to the geometrically nonlinear deformations of flat plates for cases in which plate rotations are moderate. This procedure may be applied to data from any plate analysis, based on numerical or analytical solutions. 


\section{References}

[1] Lekhnitskii, S. G. (1968). Anisotropic plates. Trans. by S. W. Tsai and T. Cheron. 2nd ed. New York: Gordon and Breach, Science Publishers.

[2] Huber, M. (1921). Theory of Plates. L'vow.

[3] Ambartsumyan, S. A. (1961). Theory of Anisotropic Shells. Moscow.

[4] Dong, S. B., Pister, K. S., and Taylor, R. L. (1962). On the theory of laminated anisotropic shells and plates. J. Aerospace Sci (29)8: 969-975.

[5] Reissner, E. and Stavsky, Y. (1961). Bending and stretching of certain types of heterogeneous aeolotropic elastic plates. Journal of Applied Mechanics (28) 402.

[6] Stavsky, Y. (1961). Bending and stretching of laminated aeolotropic plates. Proc. Am. Soc. Civil Engrs (87) 31-42.

[7] Srinivas, S. and Rao, A. K. (1970). Bending, vibration and buckling of simply supported thick orthotropic rectangular plates and laminates. International Journal of Solids and Structures (6)11: 1463-1481.

[8] Vlasov, B. (1957). On one case of bending of rectangular thick plates. Vestnik Moskovskogo Universiteta. Seriëiı Matematiki, mekhaniki, astronomii, fiziki, khimii 2: 25-34.

[9] Pagano, N. J. (1969). Exact solutions for composite laminates in cylindrical bending. Journal of Composite Materials (3)3: 398-411.

[10] Pagano, N. J. (1970). Exact solutions for rectangular bidirectional composites and sandwich plates. Journal of Composite Materials (4)1: 20. 
[11] Jones, R. M. (1975). Mechanics of Composite Materials. Ed. by B. J. Clark. Washington, D.C.: Scripta Book Company.

[12] Love, A. E. H. (1888). The small free vibrations and deformation of a thin elastic shell. Philosophical Transactions of the Royal Society of London. A (179) 491-546.

[13] Reissner, E. (1945). The effect of transverse shear deformation on the bending of elastic plates. J. appl. Mech (12)2: 69-77.

[14] Hildebrand, F. B., Reissner, E., and Thomas, G. B. (1949). Notes on the foundations of the theory of small displacements of orthotropic shells. Tech. rep. NACA TN 1833. NACA.

[15] Mindlin, R. D. (1951). Influence of Rotatory Inertia and Shear on Flexural Motions of Isotropic, Elastic Plates. J. Appl. Mech (18)1: 31-38.

[16] Girkmann, K. and Beer, R. (1958). Application of Eric Reissner's Refind Plate Theory to Orthotropic Plates. Osterr. Ingenieur-Archiv. (12) 101-110.

[17] Whitney, J. M. (1969). The effect of transverse shear deformation on the bending of laminated plates. Journal of Composite Materials (3)3: 534.

[18] Yang, P. C., Norris, C. H., and Stavsky, Y. (1966). Elastic wave propagation in heterogeneous plates. International Journal of Solids and Structures (2)4: 665-684.

[19] Whitney, J. M. and Pagano, N. J. (1970). Shear deformation in heterogeneous anisotropic plates. Journal of Applied Mechanics (37) 1031.

[20] Ashton, J. E. and Whitney, J. M. (1970). Theory of laminated plates. Vol. 4. CRC Press.

[21] Reddy, J. N. (1990). A general non-linear third-order theory of plates with moderate thickness. International Journal of Non-Linear Mechanics (25)6: 677-686.

[22] Reddy, J. N. (1993). An evaluation of equivalent-single-layer and layerwise theories of composite laminates. Composite Structures (25)1-4: 21-35.

[23] Reddy, J. N. (1984). A simple higher-order theory for laminated composite plates. Journal of Applied Mechanics (51) 745. 
[24] Reddy, J. N. (2004). Mechanics of laminated composite plates and shells: theory and analysis. CRC.

[25] Carrera, E. (1998). Evaluation of layerwise mixed theories for laminated plates analysis. AIAA Journal (36)5: 830-839.

[26] Pagano, N. J., ed. (1989). Interlaminar Response of Composite Materials. Vol. 5. Composite Materials Series. Amsterdam: Elsevier.

[27] Hyer, M. W. (1998). Stress Analysis of Fiber-Reinforced Composite Materials. Boston: McGraw-Hill.

[28] Puppo, A. H. and Evensen, H. A. (1970). Interlaminar shear in laminated composites under generalized plane stress. Journal of composite materials (4)2: 204-220.

[29] Pryor, C. W. and Barker, R. M. (1971). A finite-element analysis including transverse shear effects for applications to laminated plates. AIAA Journal (9)5: 912-917.

[30] Engblom, J. J. and Ochoa, O. O. (1986). Finite element formulation including interlaminar stress calculations. Computers $\&$ Structures (23)2: 241-249.

[31] Bhaskar, K. and Varadan, T. K. (1993). Interlaminar stresses in composite cylindrical shells under transient loads. Journal of Sound and Vibration (168)3: 469-477.

[32] Varadan, T. K. and Bhaskar, K. (1991). Bending of laminated orthotropic cylindrical shells-An elasticity approach. Composite Structures (17)2: 141-156.

[33] Barbero, E. J. and Reddy, J. N. (1990). Nonlinear analysis of composite laminates using a generalized laminated plate theory. AIAA Journal (28)11: 1987-1994.

[34] Byun, C. and Kapania, R. K. (1992). Prediction of interlaminar stresses in laminated plates using global orthogonal interpolation polynomials. AIAA Journal (30) $2740-2749$.

[35] Park, B. C., Park, J. W., and Kim, Y. H. (2003). Stress recovery in laminated composite and sandwich panels undergoing finite rotation. Composite structures (59)2: 227-235. 
[36] Reissner, E. (1948). Finite deflections of sandwich plates. Journal of the Aeronautical Sciences (15)7: 435-440.

[37] Al-Qarra, H. H. (1988). Geometrically nonlinear finite element analysis of sandwich panels. Aeronautical Journal (92)919: Finite Element Bending Analysis;Nonlinear Finite Element Analysis;Sandwich Panels; 356-364.

[38] Park, J. W. and Kim, Y. H. (1999). Predictor-corrector procedure for displacements, stresses and their sensitivity coefficients in composite panels. Journal of Composite Materials (33)13: 1222.

[39] Park, J. W. and Kim, Y. H. (2000). Predictor-corrector approach for the analysis of sandwich panels. AIAA Journal (38)8: 1489-1493.

[40] Chaudhuri, R. A. and Seide, P. (1987). An approximate semi-analytical method for prediction of interlaminar shear stresses in an arbitrarily laminated thick plate. Computers 8 Structures (25)4: 627-636.

[41] Seide, P. and Chaudhuri, R. A. (1987). Triangular finite element for analysis of thick laminated shells. International journal for numerical methods in engineering (24)8: $1563-1579$.

[42] Lee, K. and Lee, S. W. (2003). A postprocessing approach to determine transverse stresses in geometrically nonlinear composite and sandwich structures. Journal of Composite Materials (37)24: 2207.

[43] Zienkiewicz, O. C. and Zhu, J. Z. (1992). Superconvergent patch recovery and a posteriori error estimates. Part 1: the recovery technique. International Journal for Numerical Methods in Engineering (33)7: 1331-1364.

[44] Lee, K., Park, H., and Lee, S. W. (Aug. 2008). A Post-Processing Scheme to Evaluate Transverse Stresses for Composite Panels under Dynamic Loads. CMES-Computer Modeling in Engineering \&5 Sciences (32)3: 113-122. 
[45] Dakshina Moorthy, C. M. and Reddy, J. N. (1999). Recovery of interlaminar stresses and strain energy release rates in composite laminates. Finite elements in analysis and design (33)1: 1-27.

[46] Fagiano, C. et al. (2010). Interlaminar stress recovery for three-dimensional finite elements. Composites Science and Technology (70)3: 530-538.

[47] Fagiano, C., Abdalla, M. M., and Gürdal, Z. (2010). Interlaminar stress recovery of multilayer composite shell structures for three-dimensional finite elements. Finite Elements in Analysis and Design (46)12: 1122-1130.

[48] Chia, C.-Y. (1980). Nonlinear Analysis of Plates. New York: McGraw-Hill.

[49] Libai, A. and Simmonds, J. G. (1988). The Nonlinear Theory of Elastic Shells. Academic Press.

[50] Engelstad, S. P., Reddy, J. N., and Knight, N. F. (1992). Postbuckling response and failure prediction of graphite-epoxy plates loaded in compression. AIAA Journal (30)8: 2106-2113.

[51] Novozhilov, V. V. (1953). Foundations of the Nonlinear Theory of Elasticity. Dover books on mathematics. Rochester, N.Y.: Dover Publications.

[52] Hartman, T. B., Hyer, M. W., and Case, S. W. (May 2012a). Geometrically Nonlinear Stress Recovery in Composite Laminates. AIAA Journal (50)5: 1156-1168.

[53] Hartman, T. B., Hyer, M. W., and Case, S. W. (Apr. 2011). Stress Recovery in Composite Laminates. In: 52nd AIAA/ASME/ASCE/AHS/ASC Structures, Structural Dynamics, and Materials Conference. Denver, CO.

[54] LS-DYNA v971 (2007). Livermore Software Technology Corporation. Livermore, CA.

[55] Abaqus (2011). v6.11. Dassault Systèms SIMULIA Corp. Providence, RI, 02909.

[56] Hartman, T. B., Hyer, M. W., and Case, S. W. (Oct. 2012b). Geometrically Nonlinear Stress Recover in Composite Laminates Considering Inertial Effects. In: American Society for Composites 27th Technical Conference. Arlington, TX. 
[57] Amabili, M. (2008). Nonlinear vibrations and stability of shells and plates. Cambridge University Press.

[58] GNU Compiler Collection 4.1.2 (2005). Free Software Foundation, Inc.

[59] Fenlason, J. (1993). GNU gprof 2.17.50.0.6-14.el5. Free Software Foundation, Inc. 


\section{Appendix A}

\section{Development of Geometrically}

\section{Nonlinear Equilibrium Equations}

Novozhilov [51] eloquently depicts the complete transformation of the nonlinear equations of equilibrium to the linear equations of equilibrium. Within this derivation, the nonlinear equations of equilibrium considering moderate rotation are produced as an intermediate step. What follows is a summary of the work, with emphasis placed on the meaning of the various steps of the procedure, rather than the algebra contained within. The notation is that of Novozhilov, and deviates from the notation used previously in this thesis.

\section{A.1 Notation of Coordinate Systems}

Consider the undeformed flat plate shown in Figure A.1a. A global coordinate system $X$ $Y-Z$ is positioned at the geometric center of the plate, with the $X-Y$ plane coincident with the plate geometric midsurface, generally taken as the plate reference surface. Additionally, a body-centered coordinate system $x-y-z$ coincides with the global coordinate system in the undeformed configuration. The deformed configuration of the plate is shown in Figure A.1b. The global coordinate system is unchanged, but the body-centered coordinate system, originally Cartesian, is now curvilinear. 
Now consider an infinitesimal stress element located at position $P$ in the deformed configuration. The stress state referred to the global coordinate system is shown in Figure A.2a.

Following Novozhilov [51], the symbols $\xi, \eta$, and $\zeta$ are used to represent the components of stress in the $X-Y-Z$ coordinate system. Thus, $\sigma_{\xi \xi}$ represents the normal Cauchy stress on an $X$-face. Likewise, $\tau_{\zeta \eta}$ represents the Cauchy shear stress on an $Z$-face in the $Y$-direction. Similarly, stresses referred to the body coordinate system are shown in Figure A.2b. In this case, the tilde of the $\tilde{x}-\tilde{y}-\tilde{z}$ curvilinear coordinate system is omitted.

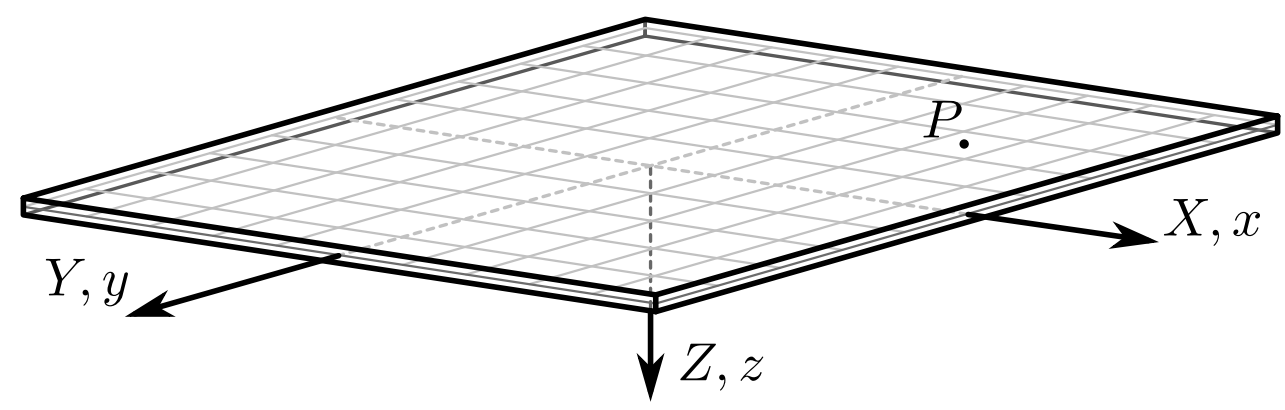

(a) Undeformed configuration.

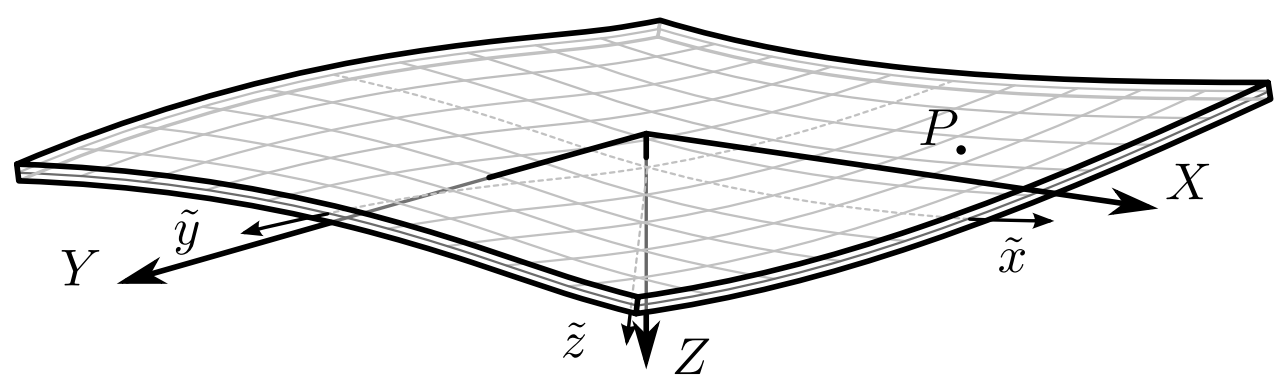

(b) Deformed configuration.

Figure A.1: Coordinate system definitions. 


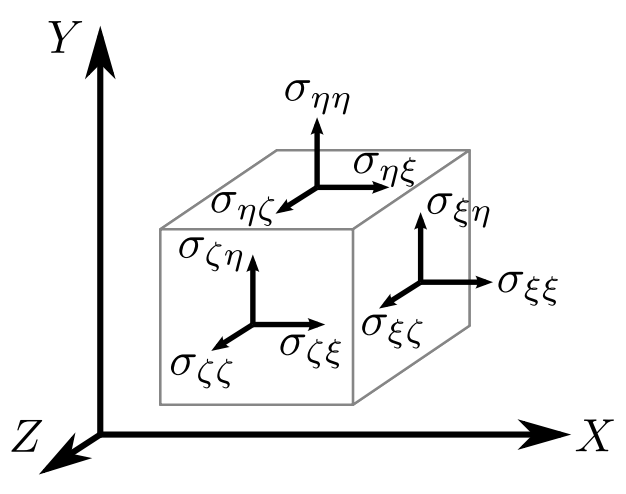

(a) Following global coordinates.

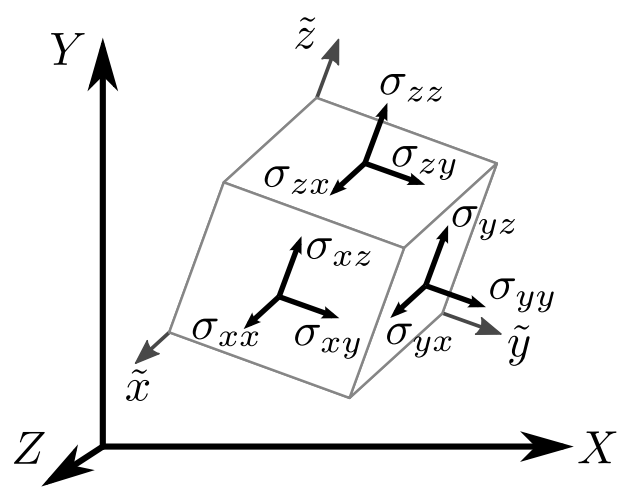

(b) Following body coordinates.

Figure A.2: Notation for stress.

\section{A.2 Balance of Linear Momentum}

From the mechanics of a continuum, the balance of linear momentum is given by

$$
\nabla \cdot \vec{\sigma}+\rho \vec{b}=\rho \vec{a}
$$

where $\vec{\sigma}$ denotes Cauchy's stress tensor, $\vec{a}$ the acceleration vector, and $\vec{b}$ the vector of specific body forces. Considering a Cartesian coordinate system with axes $X, Y, Z$, Eq. (A.1) is written as

$$
\sigma_{i j, j}+\rho b_{i}=\rho a_{i}
$$

using index notation where a comma in the subscript indicates differentiation, and summation is implied by repeated indices. Applying D'Alembert's principle, this may be stated as

$$
\sigma_{i j, j}+F_{i}^{*}=0
$$


with $F_{i}^{*}$ being the vector of specific body forces including inertial term.

\section{A.3 Balance of Linear Momentum in Global Coordinates}

As shown in Figure A.3, an element of a plate which lies at point $P$ in the undeformed configuration moves to point $P^{*}$ in the deformed configuration. Let the $\xi-\eta-\zeta$ Cartesian coordinate system in Figure A.3a be coincident with the global $X-Y-Z$ coordinate system in the deformed configuration. The position of point $P^{*}$ is then given by

$$
\begin{aligned}
& \xi=x+u(x, y, z) \\
& \eta=y+v(x, y, z) \\
& \zeta=z+w(x, y, z)
\end{aligned}
$$

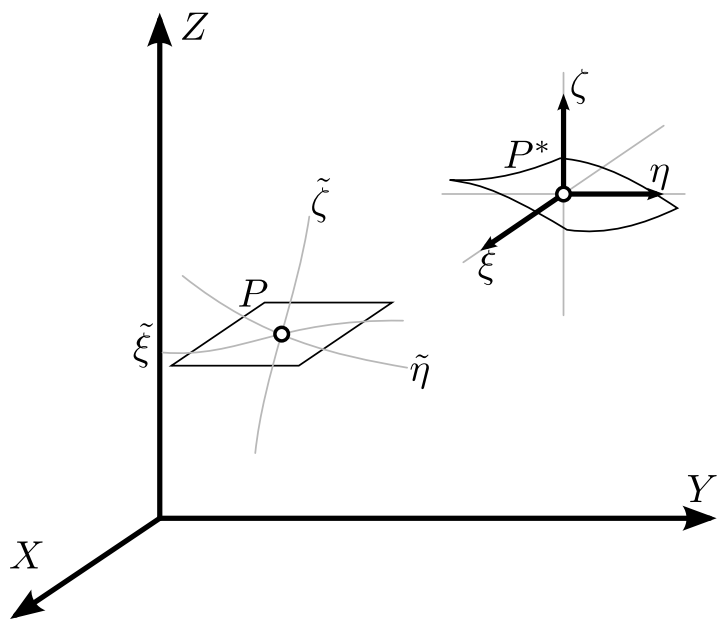

(a) Current global coordinates.

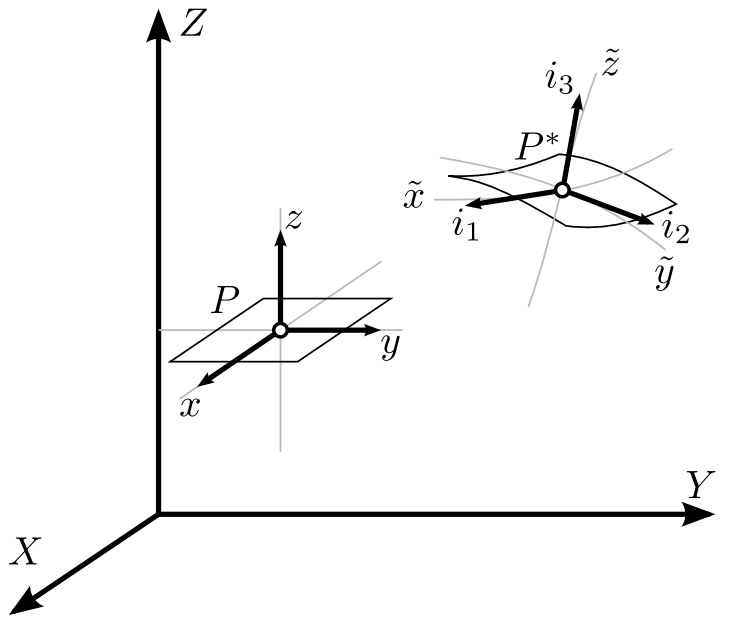

(b) Body-oriented coordinates.

Figure A.3: Notation for coordinate systems. 
where $u, v$, and $w$ are functions of the displacments of point $P^{*}$ in the global $X, Y$, and $Z$ directions, respectively. Thus, the components of stress in the $\xi-\eta-\zeta$ coordinate system are

$$
\sigma_{i j}=\left(\begin{array}{ccc}
\sigma_{\xi \xi} & \sigma_{\xi \eta} & \sigma_{\xi \zeta} \\
\sigma_{\eta \xi} & \sigma_{\eta \eta} & \sigma_{\eta \zeta} \\
\sigma_{\zeta \xi} & \sigma_{\zeta \eta} & \sigma_{\zeta \zeta}
\end{array}\right)
$$

such that $\sigma_{\xi \xi}$ refers to the stress applied in the $X$ direction on a surface whose normal is oriented in the $X$ direction in the deformed configuration. Likewise, $\sigma_{\eta \zeta}$ is the stress applied in the $Z$ direction on a surface whose normal is oriented in the $Y$ direction in the deformed configuration. Equation (A.5) represents Cauchy's stress in the global $X-Y-Z$ coordinate system. Applying this notation to the balance of linear moment of Eq. (A.3) gives

$$
\begin{aligned}
& \frac{\partial \sigma_{\xi \xi}}{\partial \xi}+\frac{\partial \sigma_{\xi \eta}}{\partial \eta}+\frac{\partial \sigma_{\xi \zeta}}{\partial \zeta}+F_{\xi}^{*}=0 \\
& \frac{\partial \sigma_{\eta \xi}}{\partial \xi}+\frac{\partial \sigma_{\eta \eta}}{\partial \eta}+\frac{\partial \sigma_{\eta \zeta}}{\partial \zeta}+F_{\eta}^{*}=0 \\
& \frac{\partial \sigma_{\zeta \xi}}{\partial \xi}+\frac{\partial \sigma_{\zeta \eta}}{\partial \eta}+\frac{\partial \sigma_{\zeta \zeta}}{\partial \zeta}+F_{\zeta}^{*}=0
\end{aligned}
$$

Applying the balance of angular momentum permits the transposition of indices of the stress components.

\section{A.4 Motivation for Coordinate Transformation}

The form of the balance of linear momentum in Eq. (A.6) is inconvenient for two reasons:

- the stress components are given in the global coordinate system.

- the differentiation is performed with respect to the global coordinate system.

The reason the former is inconvenient is that material properties of composite laminates are generally given in a body-oriented coordinate system such as given in Figure A.3b. The 
reason the latter is inconvenient is two-fold: which material points lie on the $\xi-\eta-\zeta$ coordinate axes are functions of the deformation, and data from a finite element solution are given along the $z$ coordinate axis. Therefore, not only do stresses aligned with the body-oriented coordinate system allow direct comparison to material properties, but integration along the $z$ coordinate axis is straight-forward.

It is desirable, therefore, to transform the balance of linear momentum from the global coordinates in Eq. (A.6) to the body-oriented coordinate system $x-y-z$.

\section{A.5 Transformation to Body-Oriented}

\section{Coordinate System}

If the stress components and differentiation of Eq. (A.6) is transformed to the body-oriented coordinate system $x-y$ - $z$, the balance of linear momentum becomes:

$$
\begin{array}{r}
\frac{\partial}{\partial x}\left(\left(1+e_{x x}\right) \sigma_{x x}^{*}+\left(e_{x y} / 2-\omega_{z}\right) \sigma_{x y}^{*}+\left(e_{x z} / 2+\omega_{y}\right) \sigma_{x z}^{*}\right) \\
+\frac{\partial}{\partial y}\left(\left(1+e_{x x}\right) \sigma_{y x}^{*}+\left(e_{x y} / 2-\omega_{z}\right) \sigma_{y y}^{*}+\left(e_{x z} / 2+\omega_{y}\right) \sigma_{y z}^{*}\right) \\
+\frac{\partial}{\partial z}\left(\left(1+e_{x x}\right) \sigma_{z x}^{*}+\left(e_{x y} / 2-\omega_{z}\right) \sigma_{z y}^{*}+\left(e_{x z} / 2+\omega_{y}\right) \sigma_{z z}^{*}\right) \\
+\frac{V^{*}}{V} F_{\xi}^{*}=0 \\
\frac{\partial}{\partial x}\left(\left(e_{x y} / 2+\omega_{z}\right) \sigma_{x x}^{*}+\left(1+e_{y y}\right) \sigma_{x y}^{*}+\left(e_{y z} / 2+\omega_{x}\right) \sigma_{x z}^{*}\right) \\
+\frac{\partial}{\partial y}\left(\left(e_{x y} / 2+\omega_{z}\right) \sigma_{y x}^{*}+\left(1+e_{y y}\right) \sigma_{y y}^{*}+\left(e_{y z} / 2+\omega_{x}\right) \sigma_{y z}^{*}\right) \\
+\frac{\partial}{\partial z}\left(\left(e_{x z} / 2-\omega_{y}\right) \sigma_{z x}^{*}+\left(1+e_{y y}\right) \sigma_{z y}^{*}+\left(e_{y z} / 2+\omega_{x}\right) \sigma_{z z}^{*}\right) \\
+\frac{V^{*}}{V} F_{\eta}^{*}=0
\end{array}
$$




$$
\begin{array}{r}
\frac{\partial}{\partial x}\left(\left(e_{x z} / 2-\omega_{y}\right) \sigma_{x x}^{*}+\left(e_{y z} / 2+\omega_{x}\right) \sigma_{x y}^{*}+\left(1+e_{z z}\right) \sigma_{x z}^{*}\right) \\
+\frac{\partial}{\partial y}\left(\left(e_{x z} / 2-\omega_{y}\right) \sigma_{y x}^{*}+\left(e_{y z} / 2+\omega_{x}\right) \sigma_{y y}^{*}+\left(1+e_{z z}\right) \sigma_{y z}^{*}\right) \\
+\frac{\partial}{\partial z}\left(\left(e_{x z} / 2-\omega_{y}\right) \sigma_{z x}^{*}+\left(e_{y z} / 2+\omega_{x}\right) \sigma_{z y}^{*}+\left(1+e_{z z}\right) \sigma_{z z}^{*}\right) \\
+\frac{V^{*}}{V} F_{\zeta}^{*}=0
\end{array}
$$

where $e_{i j}$ are parameters given by

$$
\begin{aligned}
e_{x x} & =\frac{\partial u}{\partial x} \\
e_{y y} & =\frac{\partial v}{\partial y} \\
e_{z z} & =\frac{\partial w}{\partial z} \\
e_{x y} & =\frac{\partial u}{\partial y}+\frac{\partial v}{\partial x} \\
e_{x z} & =\frac{\partial u}{\partial z}+\frac{\partial w}{\partial x} \\
e_{y z} & =\frac{\partial v}{\partial z}+\frac{\partial w}{\partial y}
\end{aligned}
$$

and $\omega_{x}, \omega_{y}, \omega_{z}$ are parameters given by

$$
\begin{aligned}
\omega_{x} & =\frac{1}{2}\left(\frac{\partial w}{\partial y}-\frac{\partial v}{\partial z}\right) \\
\omega_{y} & =\frac{1}{2}\left(\frac{\partial u}{\partial z}-\frac{\partial w}{\partial x}\right) \\
\omega_{z} & =\frac{1}{2}\left(\frac{\partial v}{\partial x}-\frac{\partial u}{\partial y}\right)
\end{aligned}
$$

The stress components of $\sigma_{i j}^{*}$ in Eq. (A.7) are the components of the second Piola-Kirchhoff stress tensor:

$$
\begin{aligned}
\sigma_{x x}^{*} & =\frac{S_{x}^{*}}{S_{x}} \frac{\sigma_{x x}}{1+E_{x}} \\
\sigma_{x y}^{*} & =\frac{S_{x}^{*}}{S_{x}} \frac{\sigma_{x y}}{1+E_{y}}
\end{aligned}
$$




$$
\begin{aligned}
\sigma_{x z}^{*} & =\frac{S_{x}^{*}}{S_{x}} \frac{\sigma_{x z}}{1+E_{z}} \\
\sigma_{y x}^{*} & =\frac{S_{y}^{*}}{S_{y}} \frac{\sigma_{y x}}{1+E_{x}} \\
\sigma_{y y}^{*} & =\frac{S_{y}^{*}}{S_{y}} \frac{\sigma_{y y}}{1+E_{y}} \\
\sigma_{y z}^{*} & =\frac{S_{y}^{*}}{S_{y}} \frac{\sigma_{y z}}{1+E_{z}} \\
\sigma_{z x}^{*} & =\frac{S_{z}^{*}}{S_{z}} \frac{\sigma_{z x}}{1+E_{x}} \\
\sigma_{z y}^{*} & =\frac{S_{z}^{*}}{S_{z}} \frac{\sigma_{z y}}{1+E_{y}} \\
\sigma_{z z}^{*} & =\frac{S_{z}^{*}}{S_{z}} \frac{\sigma_{z z}}{1+E_{z}}
\end{aligned}
$$

with $\sigma_{i j}$ in Eq. (A.10) being the first Piola-Kirchhoff stress components. The terms $S_{x}^{*} / S_{x}$, $S_{y}^{*} / S_{y}$, and $S_{z}^{*} / S_{z}$ are ratios of the areas after deformation to areas before deformation of areas with normals in to the $X, Y$, and $Z$ directions before deformation, respectively. The term $V^{*} / V$ is the ratio of the volume of a material element after deformation to its volume in the reference configuration. Finally, $E_{x}, E_{y}$, and $E_{z}$ are the elongations of material elements aligned with the $X, Y$, and $Z$ axes in the reference configuration. Contained within these terms are the nonlinear strain-displacement relations given by

$$
\begin{aligned}
& \varepsilon_{x x}=\frac{\partial u}{\partial x}+\frac{1}{2}\left(\left(\frac{\partial u}{\partial x}\right)^{2}+\left(\frac{\partial v}{\partial x}\right)^{2}+\left(\frac{\partial w}{\partial x}\right)^{2}\right) \\
& \varepsilon_{y y}=\frac{\partial v}{\partial y}+\frac{1}{2}\left(\left(\frac{\partial u}{\partial y}\right)^{2}+\left(\frac{\partial v}{\partial y}\right)^{2}+\left(\frac{\partial w}{\partial y}\right)^{2}\right) \\
& \varepsilon_{z z}=\frac{\partial w}{\partial z}+\frac{1}{2}\left(\left(\frac{\partial u}{\partial z}\right)^{2}+\left(\frac{\partial v}{\partial z}\right)^{2}+\left(\frac{\partial w}{\partial z}\right)^{2}\right) \\
& \varepsilon_{y z}=\frac{\partial v}{\partial z}+\frac{\partial w}{\partial y}+\frac{\partial u}{\partial y} \frac{\partial u}{\partial z}+\frac{\partial v}{\partial y} \frac{\partial v}{\partial z}+\frac{\partial w}{\partial y} \frac{\partial w}{\partial z} \\
& \varepsilon_{x z}=\frac{\partial u}{\partial z}+\frac{\partial w}{\partial x}+\frac{\partial u}{\partial x} \frac{\partial u}{\partial z}+\frac{\partial v}{\partial x} \frac{\partial v}{\partial z}+\frac{\partial w}{\partial x} \frac{\partial w}{\partial z} \\
& \varepsilon_{y z}=\frac{\partial u}{\partial y}+\frac{\partial v}{\partial x}+\frac{\partial u}{\partial x} \frac{\partial u}{\partial y}+\frac{\partial v}{\partial x} \frac{\partial v}{\partial y}+\frac{\partial w}{\partial x} \frac{\partial w}{\partial y}
\end{aligned}
$$




\section{A.5.1 Summary of Simplifications of Equilibrium Equations}

Eq. (A.7) represent the equilibrium conditions for a body with the stresses and differentiation oriented with a Cartesian coordinate system in the undeformed configuration. The straindisplacement relations are nonlinear with respect to the displacements. No restriction is made on the magnitude of either the strains or rotations of material elements.

The equations of Eq. (A.7) may be simplified by restricting the magnitude relative to unity of either the strain or rotation terms. Novozhilov proceeds such that four forms of the equilibrium equations are found, each with varying restrictions on the strains and rotations, as tabulated in Table A.1. Novozhilov [51] notes that the distinction between Eqs. (A.12) to (A.14) is negligible for massive bodies (where dimensions are of the same order of magnitude in all directions) because the assumption of small strains implies that the rotations are small. However, this is not the case for thin flexible bodies, where one dimension is vastly different from the other two.

Table A.1: Simplifications applied to equations of equilibrium in the nonlinear theory of elasticity.

\begin{tabular}{|c|c|c|c|c|}
\hline \multirow{2}{*}{ Description } & \multicolumn{2}{|c|}{ Restrictions on } & \multirow{2}{*}{$\begin{array}{c}\text { Equation in } \\
{[51]}\end{array}$} & \multirow{2}{*}{ Equation } \\
\hline & $\varepsilon_{i j}$ & $\omega_{i}$ & & \\
\hline Complete nonlinear & - & - & (II.43) & A.7 \\
\hline Small elongations and shears & $\varepsilon_{i j} \ll 1$ & - & (II.48) & A. 12 \\
\hline Moderate rotations & $\varepsilon_{i j} \ll 1$ & $\omega_{i} \ll 1$ & (II.49) & A.13 \\
\hline Classical linear elasticity & $\varepsilon_{i j} \ll 1$ & $\omega_{i} \ll \varepsilon_{i j}$ & (II.50) & A.14 \\
\hline
\end{tabular}




\section{A.5.2 Simplification for Small Strains}

If the strains are small compared to unity, Eq. (A.7) becomes

$$
\begin{array}{r}
\frac{\partial}{\partial x}\left(\left(1+e_{x x}\right) \sigma_{x x}+\left(e_{x y} / 2-\omega_{z}\right) \sigma_{x y}+\left(e_{x z} / 2+\omega_{y}\right) \sigma_{x z}\right) \\
+\frac{\partial}{\partial y}\left(\left(1+e_{x x}\right) \sigma_{y x}+\left(e_{x y} / 2-\omega_{z}\right) \sigma_{y y}+\left(e_{x z} / 2+\omega_{y}\right) \sigma_{y z}\right) \\
+\frac{\partial}{\partial z}\left(\left(1+e_{x x}\right) \sigma_{z x}+\left(e_{x y} / 2-\omega_{z}\right) \sigma_{z y}+\left(e_{x z} / 2+\omega_{y}\right) \sigma_{z z}\right) \\
+F_{\xi}^{*}=0 \\
+\frac{\partial}{\partial x}\left(\left(e_{x y} / 2+\omega_{z}\right) \sigma_{x x}+\left(1+e_{y y}\right) \sigma_{x y}+\left(e_{y z} / 2+\omega_{x}\right) \sigma_{x z}\right) \\
+\frac{\partial}{\partial y}\left(\left(e_{x y} / 2+\omega_{z}\right) \sigma_{y x}+\left(1+e_{y y}\right) \sigma_{y y}+\left(e_{y z} / 2+\omega_{x}\right) \sigma_{y z}\right) \\
+\frac{\partial}{\partial z}\left(\left(e_{x z} / 2+\omega_{z}\right) \sigma_{z x}+\left(1+e_{y y}\right) \sigma_{z y}+\left(e_{y z} / 2+\omega_{x}\right) \sigma_{z z}\right) \\
+\frac{\partial}{\partial x}\left(\left(e_{x z} / 2-\omega_{y}\right) \sigma_{x x}+\left(e_{y z} / 2+\omega_{x}\right) \sigma_{x y}+\left(1+e_{z z}\right) \sigma_{x z}\right) \\
+\frac{\partial}{\partial y}\left(\left(e_{x z} / 2-\omega_{y}\right) \sigma_{y x}+\left(e_{y z} / 2+\omega_{x}\right) \sigma_{y y}+\left(1+e_{z z}\right) \sigma_{y z}\right) \\
+\frac{\partial}{\partial z}\left(\left(e_{x z} / 2-\omega_{y}\right) \sigma_{z x}+\left(e_{y z} / 2+\omega_{x}\right) \sigma_{z y}+\left(1+e_{z z}\right) \sigma_{z z}^{*}\right) \\
+F_{\zeta}^{*}=0
\end{array}
$$

In this case, the terms $S_{x}^{*} / S_{x}, S_{y}^{*} / S_{y}, S_{z}^{*} / S_{z}$, and $V^{*} / V$ are close to unity so that they do not appear in Eq. (A.7). 


\section{A.5.3 Simplification for Moderate Rotations}

If the squares of the rotations are small compared to unity, Eq. (A.12) simplify even further to

$$
\begin{aligned}
\frac{\partial}{\partial x}\left(\sigma_{x x}-\omega_{z} \sigma_{x y}+\omega_{y} \sigma_{x z}\right) & +\frac{\partial}{\partial y}\left(\sigma_{y x}-\omega_{z} \sigma_{y y}+\omega_{y} \sigma_{y z}\right) \\
& +\frac{\partial}{\partial z}\left(\sigma_{z x}-\omega_{z} \sigma_{z y}+\omega_{y} \sigma_{z z}\right)+F_{\xi}^{*}=0 \\
\frac{\partial}{\partial x}\left(\omega_{z} \sigma_{x x}+\sigma_{x y}+\omega_{x} \sigma_{x z}\right) & +\frac{\partial}{\partial y}\left(\omega_{z} \sigma_{y x}+\sigma_{y y}+\omega_{x} \sigma_{y z}\right) \\
& +\frac{\partial}{\partial z}\left(\omega_{z} \sigma_{z x}+\sigma_{z y}+\omega_{x} \sigma_{z z}\right)+F_{\eta}^{*}=0 \\
\frac{\partial}{\partial x}\left(-\omega_{y} \sigma_{x x}+\omega_{x} \sigma_{x y}+\sigma_{x z}\right) & +\frac{\partial}{\partial y}\left(-\omega_{y} \sigma_{y x}+\omega_{x} \sigma_{y y}+\sigma_{y z}\right) \\
& +\frac{\partial}{\partial z}\left(-\omega_{y} \sigma_{z x}+\omega_{x} \sigma_{z y}+\sigma_{z z}\right)+F_{\zeta}^{*}=0
\end{aligned}
$$

These are the geometrically nonlinear equilibrium equations discussed in the body of this thesis. These are determined by considering small strains and moderate rotations. In this case, the parameters $\omega_{x}, \omega_{y}$, and $\omega_{z}$ can be considered the rotations about the global coordinate axes.

\section{A.5.4 Simplification to Classical Equilibrium Equations}

If the angles of rotation are so small that the terms in Eq. (A.13) containing them may be ignored, the classical equilibrium equations of linear elasticity are obtained:

$$
\begin{gathered}
\frac{\partial \sigma_{x x}}{\partial x}+\frac{\partial \sigma_{y x}}{\partial y}+\frac{\partial \sigma_{z x}}{\partial z}+F_{\xi}^{*}=0 \\
\frac{\partial \sigma_{x y}}{\partial x}+\frac{\partial \sigma_{y y}}{\partial y}+\frac{\partial \sigma_{z y}}{\partial z}+F_{\eta}^{*}=0 \\
\frac{\partial \sigma_{x z}}{\partial x}+\frac{\partial \sigma_{y z}}{\partial y}+\frac{\partial \sigma_{z z}}{\partial z}+F_{\zeta}^{*}=0
\end{gathered}
$$


Novozhilov notes that the criteria to move from Eq. (A.13) to Eq. (A.14) are not merely that the rotations are small, but that the terms of Eq. (A.13) in which they appear are small compared to the terms in which they do not appear. 


\section{Appendix B}

\section{Semi-Infinite Closed-Form Plate}

\section{Solutions}

Using classical lamination theory and including the effects of moderate rotations, the following equations govern plate response for the example problems:

For $-a / 2 \leq x \leq-\alpha a / 2$ and $\alpha a / 2 \leq x \leq a / 2$ (left and right unloaded sections of plate),

$$
\begin{gathered}
\frac{\mathrm{d} N_{x}}{\mathrm{~d} x}=0 \\
\frac{\mathrm{d}^{2} M_{x}}{\mathrm{~d} x^{2}}+\frac{d}{\mathrm{~d} x}\left(N_{x} \frac{\mathrm{d} w^{\circ}}{\mathrm{d} x}\right)=0
\end{gathered}
$$

For $-\alpha a / 2 \leq x \leq \alpha a / 2$ (central loaded section of plate),

$$
\begin{gathered}
\frac{\mathrm{d} N_{x}}{\mathrm{~d} x}+t_{0}=0 \\
\frac{\mathrm{d}^{2} M_{x}}{\mathrm{~d} x^{2}}+\frac{\mathrm{d}}{\mathrm{d} x}\left(N_{x} \frac{\mathrm{d} w^{\circ}}{\mathrm{d} x}\right)+t_{0} \frac{\mathrm{d} w^{\circ}}{\mathrm{d} x}+q_{0}=0
\end{gathered}
$$


where

$$
\begin{gathered}
N_{x}=A_{11}\left(\frac{\mathrm{d} u^{\circ}}{\mathrm{d} x}+\frac{1}{2}\left(\frac{\mathrm{d} w^{\circ}}{\mathrm{d} x}\right)^{2}\right) \\
M_{x}=-D_{11} \frac{\mathrm{d}^{2} w^{\circ}}{\mathrm{d} x^{2}}
\end{gathered}
$$

and standard nomenclature has been used. The boundary conditions for this classical lamination theory formulation of the two example problems are

$$
u^{\circ}( \pm a / 2)=0, \quad w^{\circ}( \pm a / 2)=0, \quad M_{x}( \pm a / 2)=0
$$

In addition, continuity of the displacements $\left(u^{\circ}\right.$ and $\left.w^{\circ}\right)$, rotations $\left(\omega_{y}^{\circ}\right)$, and the force, moment, and effective shear force resultants $\left(N_{x}, M_{x}\right.$, and $\left.\mathrm{d} M_{x} / \mathrm{d} x\right)$ must be enforced at $x= \pm \alpha a / 2$. Those twelve conditions plus the six boundary conditions of Eq. (B.4) lead to a solution to the problem. The transverse loading case $\left(q_{0} \neq 0, t_{0}=0\right)$ and the shear loading case $\left(q_{0}=0, t_{0} \neq 0\right)$ are considered separately, mainly to be able to better discuss the features of the stress state and the comparisons among the plate finite element, benchmark three-dimensional finite element, and closed-form solutions for the interlaminar stresses $\tau_{x z}$ and $\sigma_{z z}$. Also, the transverse loading case can be solved in closed form, whereas the shear loading case cannot be solved in closed form, so separation of the two load cases provides an operational advantage as well. 


\section{B.1 Solutions for Loading Cases}

\section{B.1.1 Transverse Loading Case $\left(q_{0} \neq 0, t_{0}=0\right)$}

For the transverse loading case,

$$
\begin{aligned}
& u^{\circ}(x)= \begin{cases}\frac{N_{0}}{A_{11}}\left(x+\frac{a}{2}\right)-\frac{1}{2} \int\left(\frac{\partial w^{\circ}}{\partial x}\right)^{2} \mathrm{~d} x & -\frac{a}{2} \leq x<-\frac{\alpha a}{2} \\
\frac{N_{0}}{A_{11}} x-\frac{1}{2} \int\left(\frac{\partial w^{\circ}}{\partial x}\right)^{2} \mathrm{~d} x & -\frac{\alpha a}{2} \leq x \leq \frac{\alpha a}{2} \\
\frac{N_{0}}{A_{11}}\left(x-\frac{a}{2}\right)-\frac{1}{2} \int\left(\frac{\partial w^{\circ}}{\partial x}\right)^{2} \mathrm{~d} x+C & \frac{\alpha a}{2}<x \leq \frac{a}{2}\end{cases} \\
& w^{\circ}(x)=\frac{D_{11} q_{0}}{N_{0}^{2}}\left\{\begin{array}{rlr}
\frac{\alpha a}{2}\left(\frac{a}{2}+x\right) \beta^{2} & \\
-\sinh \left(\frac{a \alpha \beta}{2}\right)\left(\sinh (x \beta)+\cosh (x \beta) \tanh \left(\frac{a \beta}{2}\right)\right) & -\frac{a}{2} \leq x<-\frac{\alpha a}{2} \\
-\left(\cosh (x \beta) \cosh \left(\frac{a(\alpha-1) \beta}{2}\right) \operatorname{sech}\left(\frac{a \beta}{2}\right)-1\right) & -\frac{\alpha a}{2} \leq x \leq \frac{a \alpha}{2} \\
\frac{\alpha a}{2}\left(\frac{a}{2}-x\right) \beta^{2} & \\
+\sinh \left(\frac{a \alpha \beta}{2}\right)\left(\sinh (x \beta)-\cosh (x \beta) \tanh \left(\frac{a \beta}{2}\right)\right) & \frac{a \alpha}{2}<x \leq \frac{a}{2}
\end{array}\right.
\end{aligned}
$$

in which

$$
\begin{gathered}
\beta=\sqrt{\frac{N_{0}}{D_{11}}} \\
C=\frac{1}{2}\left(\frac{q_{0}}{4 \beta N_{0}}\right)^{2} \operatorname{sech}\left(\frac{a \beta}{2}\right)^{2} a\left(a^{2} \alpha^{2} \beta^{2}+a^{2} \alpha^{2} \beta^{2} \cosh (a \beta)+2 \cosh (a \alpha \beta)-2\right)
\end{gathered}
$$

In Eq. (B.5) the value of the integration constant $N_{0}$ is still not known but will be found when all boundary and continuity conditions are enforced. 


\section{B.1.2 Shear Loading Case $\left(q_{0}=0, t_{0} \neq 0\right)$}

For the shear loading case, a Rayleigh-Ritz technique is used to obtain approximate solutions for the displacements $u^{\circ}(x)$ and $w^{\circ}(x)$. This actually broadens the applicability of the stress recovery procedure to cases where exact closed-form solutions are not obtainable, but closedform algebraic expressions for approximate solutions are. The forms of approximate solutions for the displacements in the three sections of the plate are assumed as follows: For $-a / 2 \leq x \leq-\alpha a / 2$ (left unloaded section of plate),

$$
\begin{aligned}
& u^{\circ}(x)=(a / 2+x) \sum_{i=0}^{\mathcal{N}_{1}-1}\left(A_{1 i} x^{i}\right) \\
& w^{\circ}(x)=(a / 2+x) \sum_{i=0}^{\mathcal{N}_{2}-1}\left(B_{1 i} x^{i}\right)
\end{aligned}
$$

For $-\alpha a / 2 \leq x \leq \alpha a / 2$ (central loaded section of plate),

$$
\begin{gathered}
u^{\circ}(x)=\sum_{i=0}^{\mathcal{N}_{3}-1}\left(A_{2 i} x^{i}\right) \\
w^{\circ}(x)=\sum_{i=0}^{\mathcal{N}_{4}-1}\left(B_{2 i} x^{i}\right)
\end{gathered}
$$

For $\alpha a / 2 \leq x \leq a / 2$ (right unloaded section of plate),

$$
\begin{gathered}
u^{\circ}(x)=(-a / 2+x) \sum_{i=0}^{\mathcal{N}_{5}-1}\left(A_{3 i} x^{i}\right) \\
w^{\circ}(x)=(-a / 2+x) \sum_{i=0}^{\mathcal{N}_{6}-1}\left(B_{3 i} x^{i}\right)
\end{gathered}
$$


where $\mathcal{N}_{i}$ is uniquely chosen for each displacement expression and $A_{i j}$ and $B_{i j}$ are unknown coefficients to be determined by application of the Rayleigh-Ritz technique. (Note: the $A_{i j}$ in Eqs. (B.7) to (B.9) are not to be confused with the extensional stiffnesses $A_{i j}$.) It is important to note that the kinematic boundary conditions at $x=-a / 2$ in Eq. (B.4) are satisfied with the from of Eq. (B.7), as required by the Rayleigh-Ritz technique, as are the kinematic boundary conditions at $x=+a / 2$ with the form of Eq. (B.9). Also to be noted is that not all coefficients $A_{i j}$ and $B_{i j}$ in the above six expressions are independent. As mentioned, the displacements and rotations must be continuous between plate sections. This imposes six constraints on the $\mathcal{N}$ unknown coefficients. Here the six continuity equations are used to express six unknown coefficients in terms of the other $(\mathcal{N}-6)$.

\section{B.2 Details of Semi-Infinite Rayleigh-Ritz Solutions}

For the results discussed based on the Rayleigh-Ritz technique, the number of coefficients $\mathcal{N}_{i}$ in the assumed displacement forms of Eqs. (B.7) to (B.9) are given in Table B.1. The particular values of $A_{i j}$ and $B_{i j}$ in Eqs. (B.7) to (B.9) are then determined uniquely by application of the Rayleigh-Ritz technique for the given plate geometry, material properties, and shear load level $t_{o}$. Values of the coefficients of the solutions for the isotropic, cross-ply, and angle-ply plates for the shear load case $q_{0}=0, t_{0} \neq 0$ are given in Table B.2.

Table B.1: Number of coefficients chosen for polynomials in Rayleigh-Ritz technique.

\begin{tabular}{ccc}
\hline Section & $\mathcal{N}$ in $u^{\circ}(x)$ & $\mathcal{N}$ in $w^{\circ}(x)$ \\
\hline$-a / 2<x<-\alpha a / 2$ & 5 & 7 \\
$-\alpha a / 2<x<\alpha a / 2$ & 6 & 7 \\
$\alpha a / 2<x<a / 2$ & 5 & 7 \\
\hline
\end{tabular}


Table B.2: Coefficients of Rayleigh-Ritz solutions.

\begin{tabular}{|c|c|c|c|c|c|c|c|}
\hline \multirow[b]{2}{*}{$i$} & \multirow[b]{2}{*}{$j$} & \multicolumn{2}{|c|}{ Isotropic Plate } & \multicolumn{2}{|c|}{ Cross-ply Plate } & \multicolumn{2}{|c|}{ Angle-ply Plate } \\
\hline & & $A_{i j}$ & $B_{i j}$ & $A_{i j}$ & $B_{i j}$ & $A_{i j}$ & $B_{i j}$ \\
\hline 1 & 0 & $+1.233 \mathrm{E}-4$ & $+7.176 \mathrm{E}-3$ & $+2.848 \mathrm{E}-6$ & $+1.156 \mathrm{E}-3$ & $+2.785 \mathrm{E}-6$ & $+1.238 \mathrm{E}-3$ \\
\hline 1 & 1 & $-7.892 \mathrm{E}-5$ & $+9.158 \mathrm{E}-3$ & $-1.526 \mathrm{E}-6$ & $+1.154 \mathrm{E}-3$ & $-1.915 \mathrm{E}-6$ & $+1.350 \mathrm{E}-3$ \\
\hline 1 & 2 & $-1.959 \mathrm{E}-4$ & $+1.651 \mathrm{E}-2$ & $-3.739 \mathrm{E}-6$ & $+2.271 \mathrm{E}-3$ & $-4.971 \mathrm{E}-6$ & $+2.878 \mathrm{E}-3$ \\
\hline 1 & 3 & $-2.634 \mathrm{E}-4$ & $+1.747 \mathrm{E}-2$ & $-5.000 \mathrm{E}-6$ & $+2.741 \mathrm{E}-3$ & $-6.987 \mathrm{E}-6$ & $+3.865 \mathrm{E}-3$ \\
\hline 1 & 4 & $-1.641 \mathrm{E}-4$ & $+1.588 \mathrm{E}-2$ & $-3.130 \mathrm{E}-6$ & $+2.686 \mathrm{E}-3$ & $-4.454 \mathrm{E}-6$ & $+4.043 \mathrm{E}-3$ \\
\hline 1 & 5 & & $+8.951 \mathrm{E}-3$ & & $+1.663 \mathrm{E}-3$ & & $+2.690 \mathrm{E}-3$ \\
\hline 1 & 6 & & $+3.266 \mathrm{E}-3$ & & $+6.165 \mathrm{E}-4$ & & $+1.013 \mathrm{E}-3$ \\
\hline 2 & 0 & $+5.724 \mathrm{E}-5$ & $+3.479 \mathrm{E}-3$ & $+1.322 \mathrm{E}-6$ & $+5.622 \mathrm{E}-4$ & $+1.301 \mathrm{E}-6$ & $+6.013 \mathrm{E}-4$ \\
\hline 2 & 1 & $+6.465 \mathrm{E}-6$ & +8. & $+2.696 \mathrm{E}-7$ & & +2.797 & \\
\hline 2 & 2 & $-4.062 \mathrm{E}-4$ & $-1.600 \mathrm{E}-2$ & $-9.184 \mathrm{E}-6$ & $-2.565 \mathrm{E}-3$ & $-8.048 \mathrm{E}-6$ & $-2.718 \mathrm{E}-3$ \\
\hline 2 & 3 & $+6.011 \mathrm{E}-4$ & $-9.136 \mathrm{E}-2$ & $+1.246 \mathrm{E}-5$ & $-1.344 \mathrm{E}-2$ & $+1.538 \mathrm{E}-5$ & $-1.480 \mathrm{E}-2$ \\
\hline 2 & 4 & $-2.170 \mathrm{E}-3$ & $-6.754 \mathrm{E}-3$ & $-5.090 \mathrm{E}-5$ & $-1.425 \mathrm{E}-3$ & $-5.911 \mathrm{E}-5$ & $-2.071 \mathrm{E}-3$ \\
\hline 2 & 5 & $-7.637 \mathrm{E}-3$ & $+1.176 \mathrm{E}-2$ & $-1.671 \mathrm{E}-4$ & $+2.275 \mathrm{E}-3$ & $-2.035 \mathrm{E}-4$ & $+1.321 \mathrm{E}-3$ \\
\hline 2 & 6 & & $+1.966 \mathrm{E}-1$ & & $+3.471 \mathrm{E}-2$ & & $+4.291 \mathrm{E}-2$ \\
\hline 3 & 0 & $-1.283 \mathrm{E}-4$ & $-6.744 \mathrm{E}-3$ & $-2.961 \mathrm{E}-6$ & $-1.094 \mathrm{E}-3$ & $-2.919 \mathrm{E}-6$ & $-1.168 \mathrm{E}-3$ \\
\hline 3 & 1 & $+4.375 \mathrm{E}-5$ & $-3.678 \mathrm{E}-2$ & $+7.227 \mathrm{E}-7$ & $-5.615 \mathrm{E}-3$ & $+9.627 \mathrm{E}-7$ & $-6.114 \mathrm{E}-3$ \\
\hline 3 & 2 & $-1.365 \mathrm{E}-3$ & $+2.117 \mathrm{E}-2$ & $-3.195 \mathrm{E}-5$ & $+2.885 \mathrm{E}-3$ & $-3.777 \mathrm{E}-5$ & $+3.305 \mathrm{E}-3$ \\
\hline 3 & 3 & $+2.272 \mathrm{E}-3$ & $+2.864 \mathrm{E}-2$ & $+5.231 \mathrm{E}-5$ & $+4.956 \mathrm{E}-3$ & $+6.245 \mathrm{E}-5$ & $+5.184 \mathrm{E}-3$ \\
\hline 3 & 4 & $-7.340 \mathrm{E}-4$ & $-8.335 \mathrm{E}-3$ & $-1.515 \mathrm{E}-5$ & $-1.326 \mathrm{E}-3$ & $-1.909 \mathrm{E}-5$ & $-1.645 \mathrm{E}-3$ \\
\hline 3 & 5 & & $-6.601 \mathrm{E}-3$ & & $-1.257 \mathrm{E}-3$ & & $-9.803 \mathrm{E}-4$ \\
\hline 3 & 6 & & $+1.973 \mathrm{E}-3$ & & $+3.719 \mathrm{E}-4$ & & $+2.747 \mathrm{E}-4$ \\
\hline
\end{tabular}

\section{B.3 Application of the Stress Recovery Procedure}

From the solutions for the displacement components from either load case, the needed intralaminar stresses $\sigma_{x x}$ and $\tau_{x y}$ and their partial derivatives are available for substitution into the iterative stress recovery procedure to determine the interlaminar stresses for the two example problems at the two span-wise locations identified in Figures 4.2a and 4.2b. 


\section{Appendix C}

\section{Justification for Switching to Abaqus for Dynamic Plate Solutions}

While excellent agreement between the LS-DYNA plate and full three-dimensional solutions was obtained for the static implicit analyses, this agreement was not seen for the dynamic explicit analyses. However, good agreement was found between the LS-DYNA full threedimensional solution and a plate solution implemented in the Abaqus finite element software using a dynamic explicit analysis.

The purpose of this appendix is to present evidence of the differences observed as the reason from moving from LS-DYNA to Abaqus for obtaining finite element data for use in the stress recovery procedure for the dynamic loading cases.

A square, simply-supported, eight-layer laminated plate is modeled, as described in Chapter 6. A uniform distributed pressure load is applied to the top surface of the plate at a rate of $3.0 \mathrm{GPas}^{-1}$. Figure C.1a depicts the deflection history of the center of the plate up to a time of $0.5 \mathrm{~ms}$, at which point the simulation was terminated. The response at the mid-surface of the LS-DYNA three-dimensional (solid element) solution is shown at discrete points, while data from the LS-DYNA plate solution and the Abaqus plate solution are shown as solid and dashed lines, respectively. Note the agreement between three solutions 


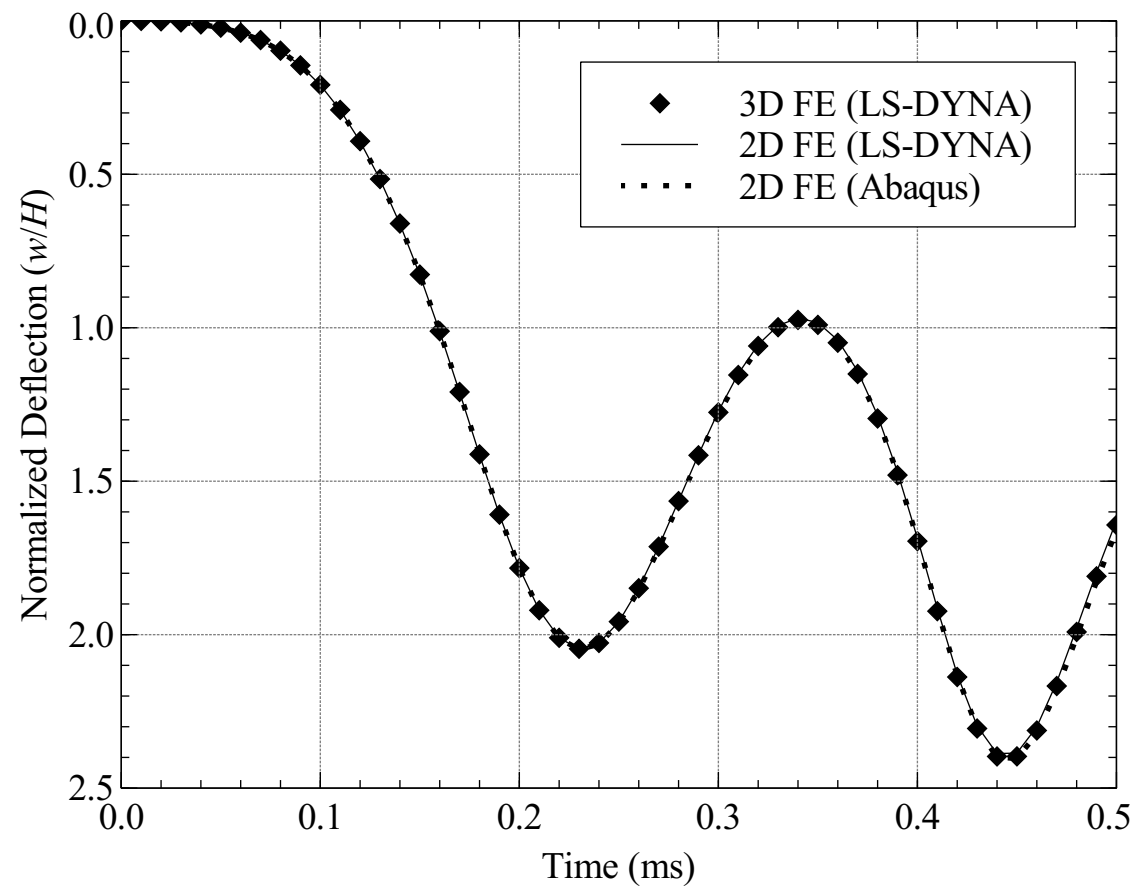

(a) Deflection.

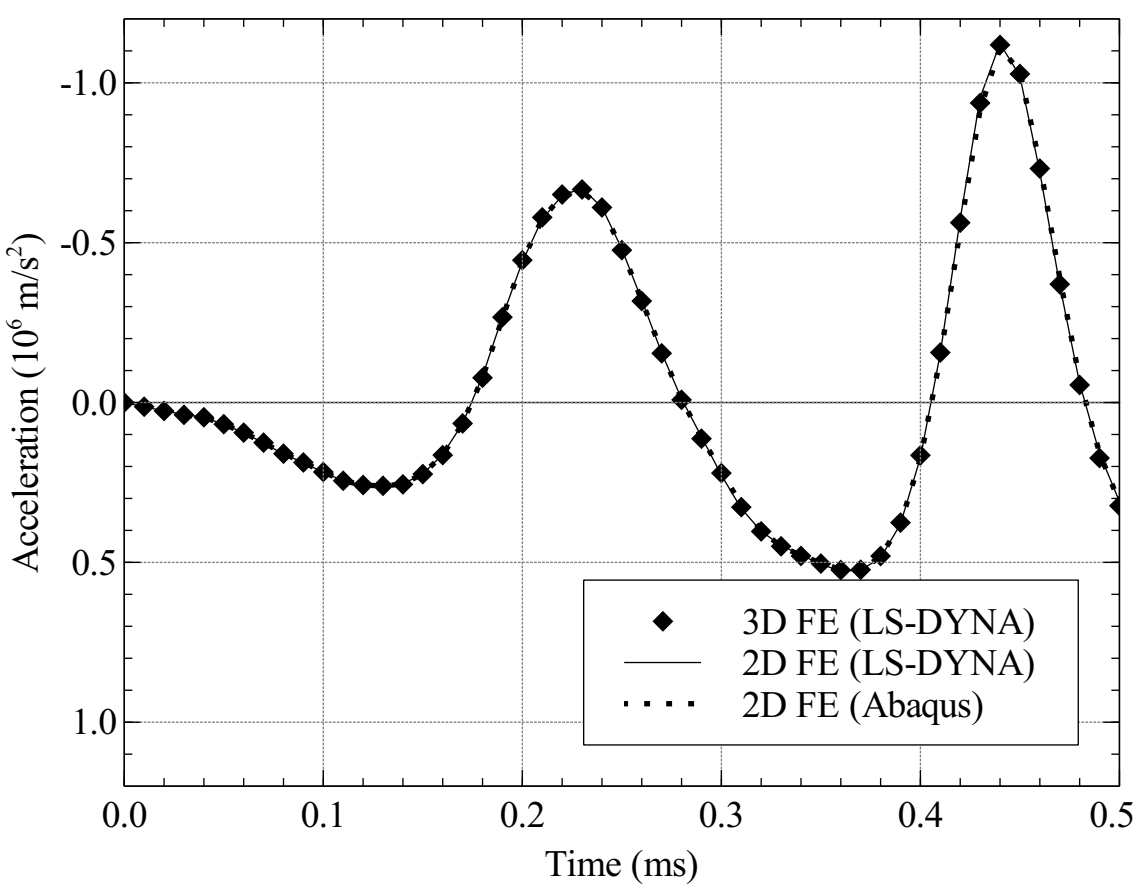

(b) Acceleration.

Figure C.1: Kinematics of ramp loading using various finite element analysis software. 
for the time interval considered.

Figure C.1b shows the acceleration history of the mid-surface at the center of the plate, with the same representations used for the different solutions as in Figure C.1a. Again, good agreement is observed between all three solutions.

Deflections at other points in the plate certainly differ from the center point, and the agreement between the solutions may change as a function of location in the plate. In order to asses the deflections of the entire plate, the deflection data from the LS-DYNA plate and three-dimensional solutions are compared in Figure C.2 at time $t=0.3 \mathrm{~ms}$. The contours represent the absolute value of the percent difference of the deflection data from the plate solution compared to the three-dimensional solution. At the center of the plate $(x / a=0$, $y / b=0)$, the difference is less than $1 \%$. From that point, each contour line delineates a change of $1 \%$, with a solid bold line marking $5 \%$ difference and a dashed bold line marking $10 \%$ difference.

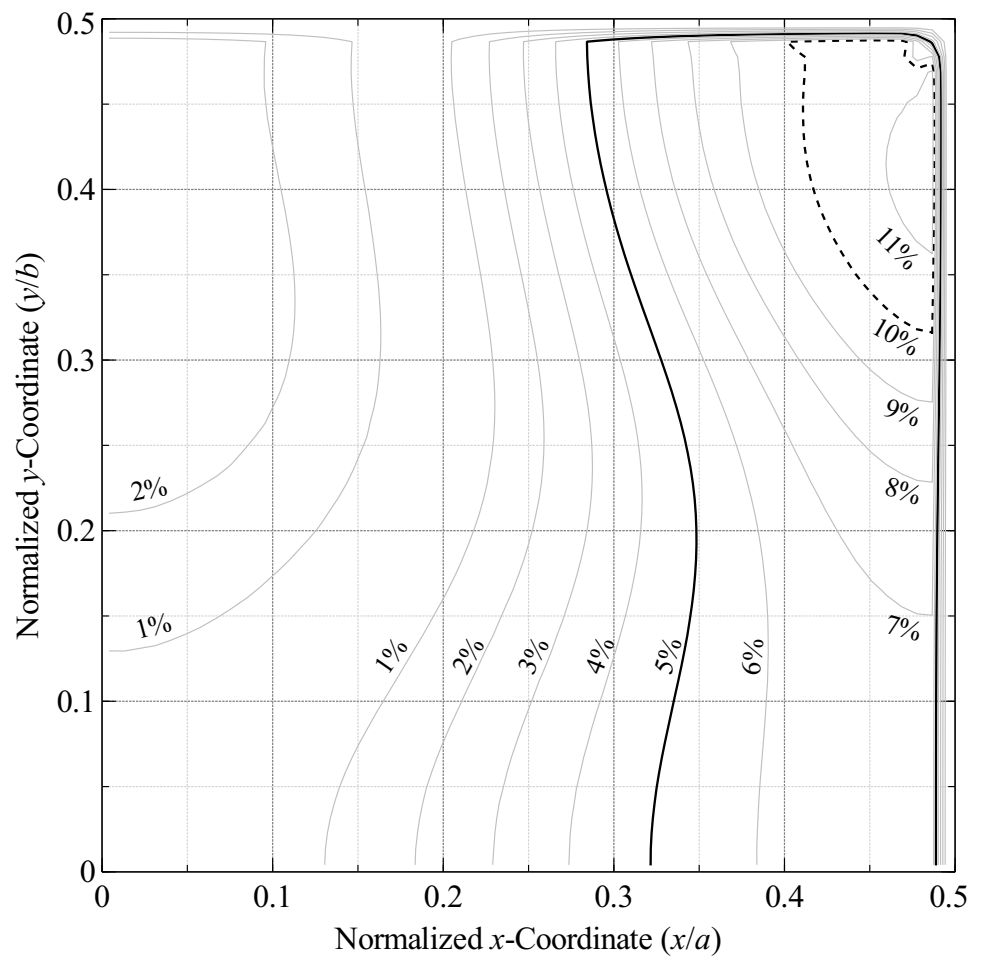

Figure C.2: Difference between solid and plate. 
To depict this differently, the deflection profiles of the LS-DYNA plate and three-dimensional solutions at $y / b=0.25$ (a section running through the quarter-point) are given in Figure C.3. While a discrepancy exists between the solutions, the difference is small.

The data shown thus far suggest that the plate and three-dimensional solutions from LS-DYNA are quite comparable. In fact, the kinematics of the plate solutions do appear to follow closely the response predicted by the three-dimensional solution. However, the stress data do not share this agreement.

The intralaminar normal stress $\sigma_{x x}$ near the top surface $(z / H=-0.4844)$ at the quarterpoint $(x / a=0.25, y / b=0.25)$ is shown for the duration of the simulation in Figure C.4. For

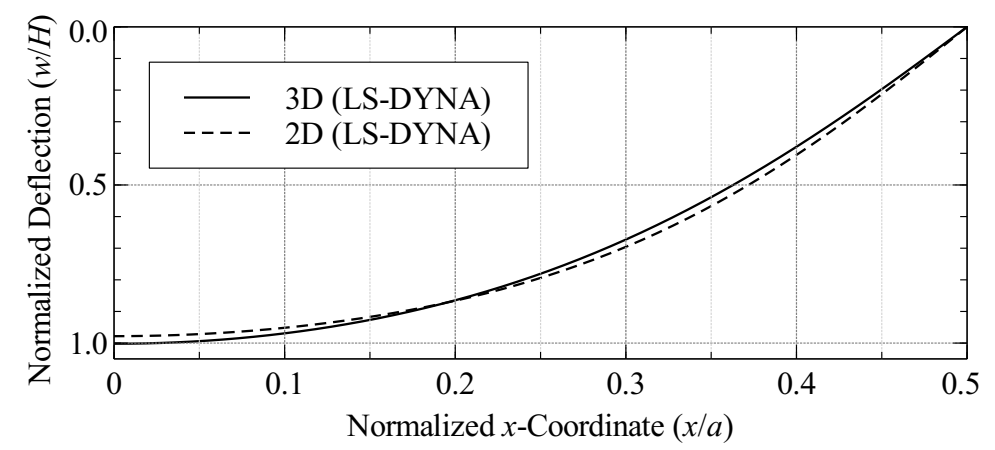

Figure C.3: Difference between solid and plate at $y / b=0.25$.

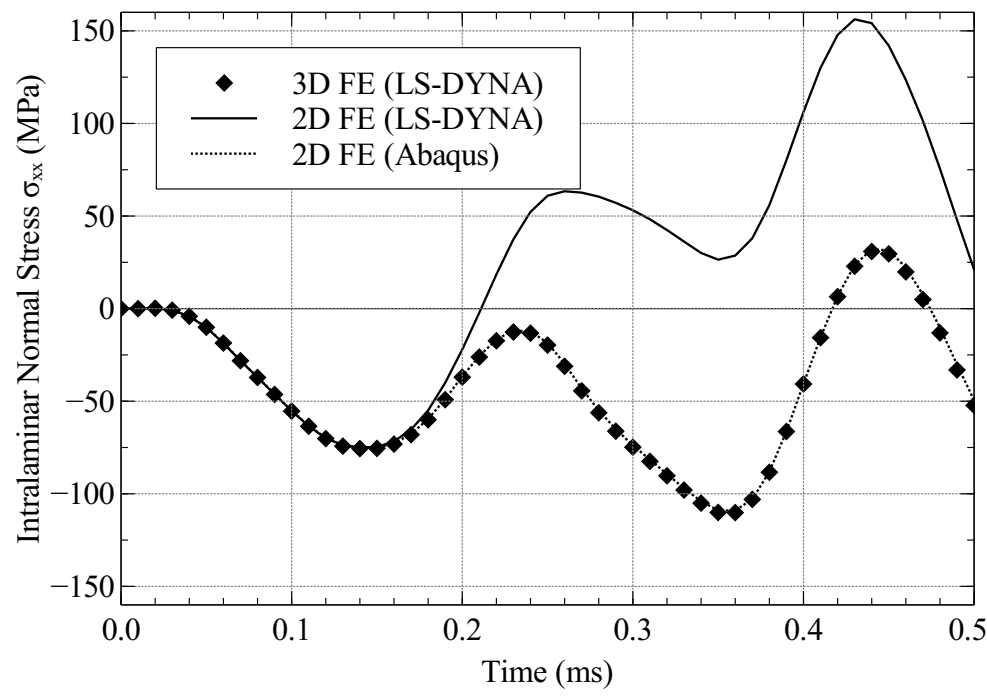

Figure C.4: Stress history at quarter-point. 
small deflections, the value of this stress should be negative, corresponding to compressive stresses due to bending. With increasing deflections, the stress may be less negative (or even positive), as membrane stresses increase. Indeed, the predicted stress from the LSDYNA three dimensional solution and both plate solutions (via LS-DYNA and Abaqus) are initially compressive and show good agreement. However, while the Abaqus plate solution tracks closely to the three-dimensional solution for the entire time-history, the LS-DYNA plate solution begins to diverge around $t=0.16 \mathrm{~ms}$. From that point, the difference in the LS-DYNA plate solution compared to the other two solutions becomes quite large.

At $t=0.3 \mathrm{~ms}$, the distribution of $\sigma_{x x}$ through the laminate thickness at the quarter-point is shown in Figure C.5 for all three solutions. Once again, the LS-DYNA three-dimensional and Abaqus plate solutions show good agreement, while the LS-DYNA plate distribution is quite different.

For these reasons, the results for the dynamic stress recovery are shown using the Abaqus finite element software to obtain in-plane stress data.

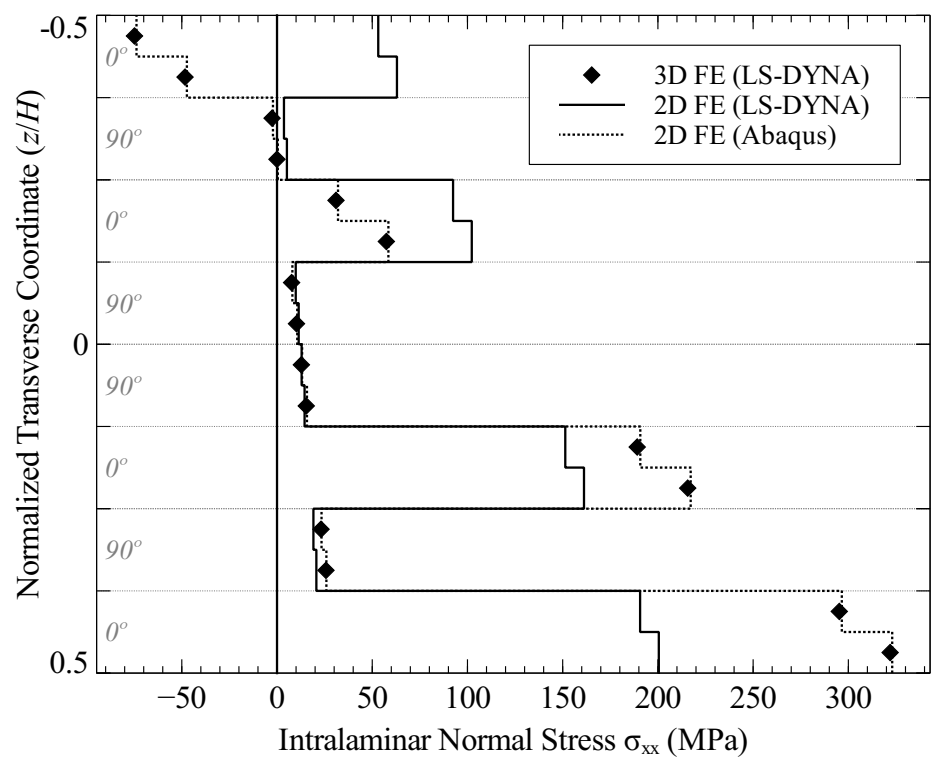

Figure C.5: Stress distribution at quarter-point. 


\section{Appendix D}

\section{Permissions for Copyrighted Content}

Permission to use third party copyrighted material included in this dissertation has been

obtained from the copyright owners. Details of the granted permissions are presented within this chapter.

(This area intentionally blank.) 


\section{D.1 Reprint of Figure 2.1.}

Permission to reprint Figure 2.1 was granted by SAGE Publications for use in this thesis per the following document:

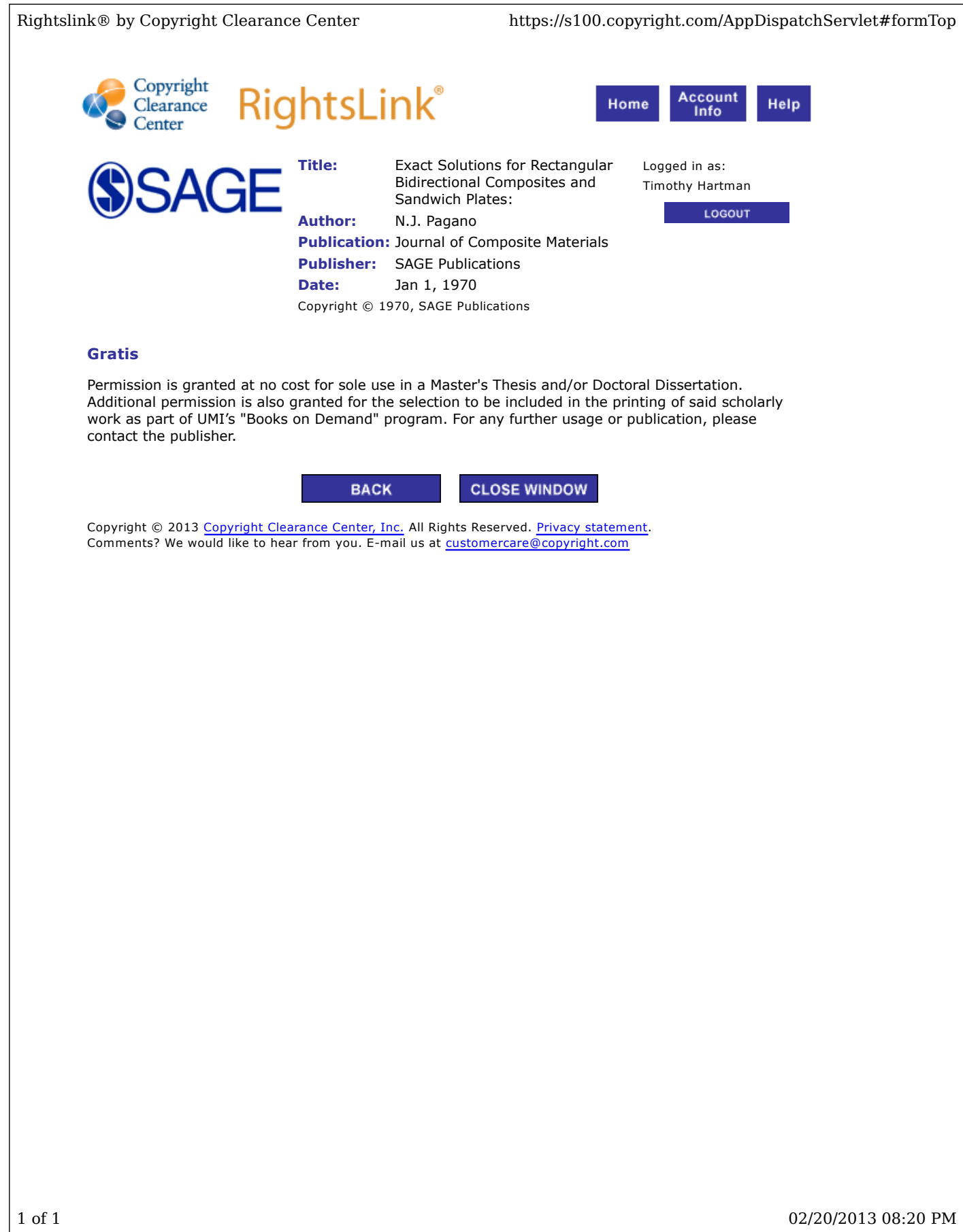




\section{D.2 Reprint of Figure 2.2.}

Permission to reprint Figure 2.2 was granted by the Taylor \& Francis Group for use in this thesis per the following document:

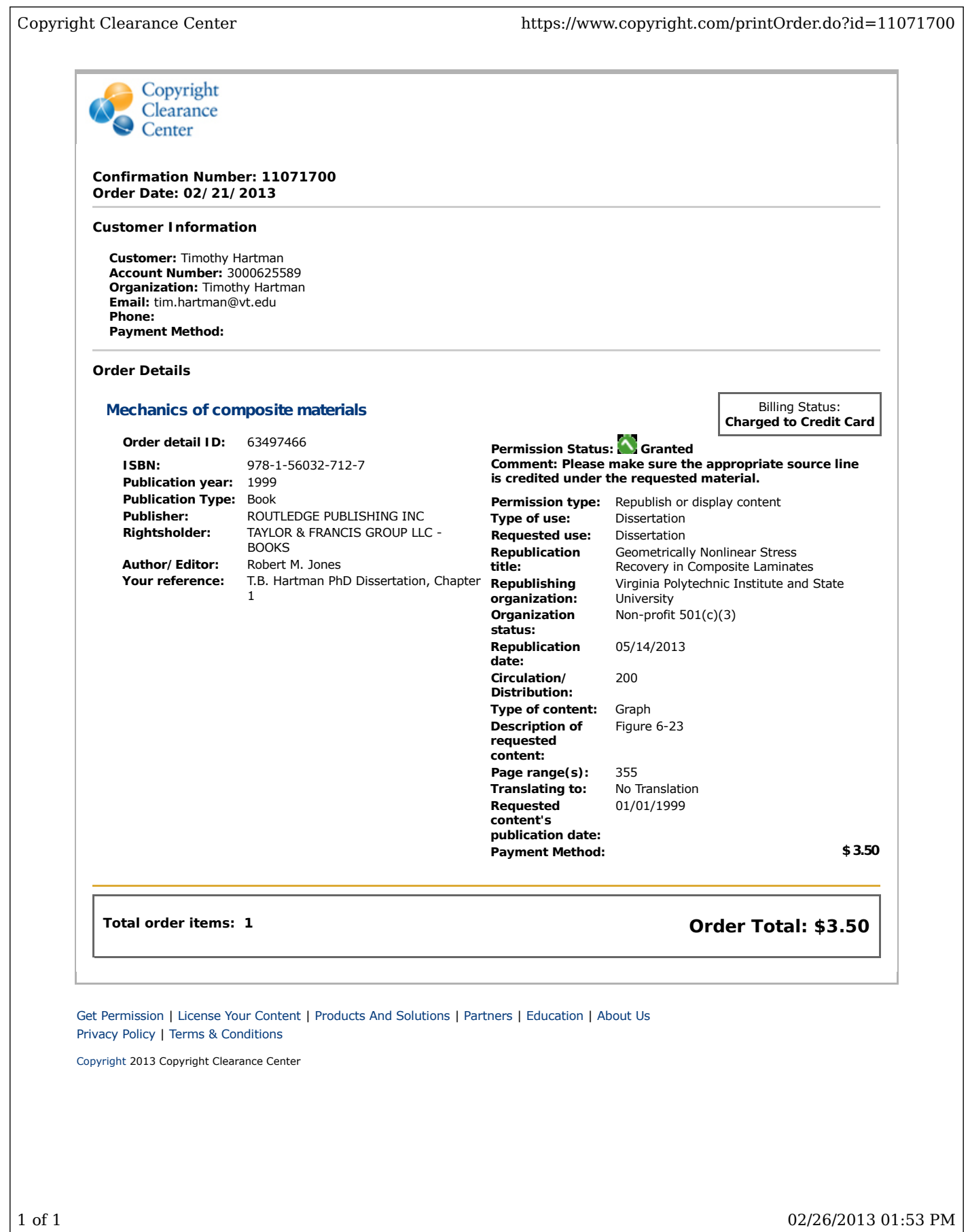

